


\title{
A liquid foam-bed photobioreactor for microalgae production
}

\author{
Ágnes Jánoska
}




\section{Thesis committee}

\section{Promotor}

Prof. Dr R.H. Wijffels

Professor of Bioprocess Engineering

Wageningen University \& Research

\section{Co-Promotor}

Dr M.G.J. Janssen

Assistant Professor, Bioprocess Engineering Group

Wageningen University \& Research

\section{Other members}

Dr D.W.F. Brilman, University of Twente, Enschede, Netherlands

Prof. Dr. C.J.N. Buisman, Wetsus, Leeuwarden / Wageningen University \& Research

Prof. Dr J. Pruvost, University of Nantes, France

Dr E.J.A. Roebroeck, LGem, Voorhout, Netherlands

This research was conducted under the auspices of the Graduate School VLAG (Advanced studies in Food technology, Agrobiotechnology, Nutrition and Health Sciences) 


\section{A liquid foam-bed photobioreactor for microalgae production}

Ágnes Jánoska

\section{Thesis}

submitted in fulfilment of the requirement for the degree of doctor at Wageningen University

by the authority of the Rector Magnificus

Prof. Dr A.P.J. Mol, in the presence of the

Thesis Committee appointed by the Academic Board

to be defended in public

on Friday 15 June 2018

at 1:30 p.m. in the Aula. 
Á. Jánoska

A liquid foam-bed photobioreactor for microalgae cultivation, 218 pages.

PhD thesis, Wageningen University, Wageningen, NL (2018)

With references, with summary in English

ISBN: 978-94-6343-268-9

DOI $10.18174 / 445175$ 




\section{Contents}

\section{Chapter 1}

Introduction 9

\section{Chapter 2}

Development of a liquid foam-bed photobioreactor for microalgae cultivation

\section{Chapter 3}

Surfactant selection for a liquid foam-bed photobioreactor

\section{Chapter 4}

Improved liquid foam-bed photobioreactor design for microalgae cultivation

\section{Chapter 5}

Potential of a liquid foam-bed photobioreactor for microalgae cultivation

\section{Chapter 6}

General discussion

Summary

Acknowledgments

About the author

List of publications 

N.

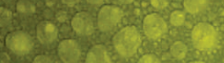

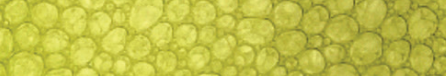

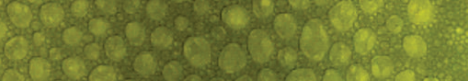

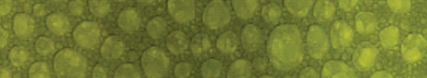

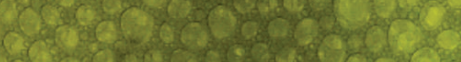

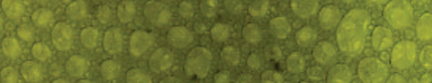

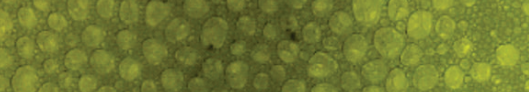

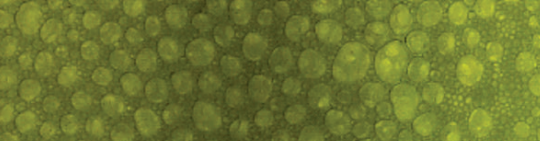
(c) 8

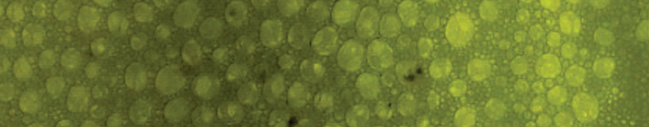

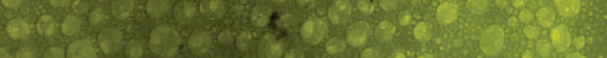
C.7.

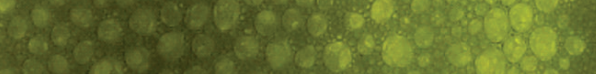
13.

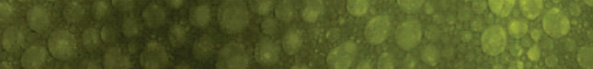
Q6es paras

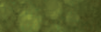

Sor. 20. W.

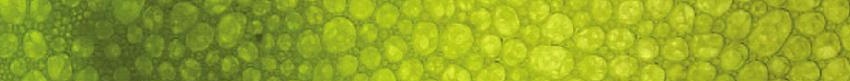

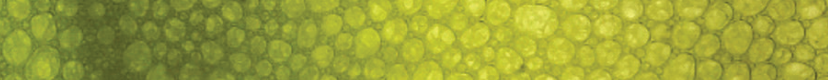

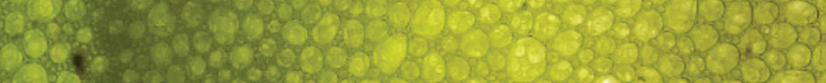

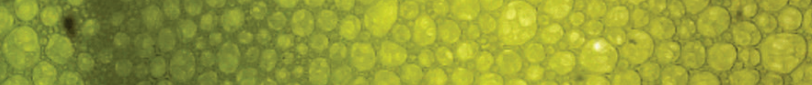

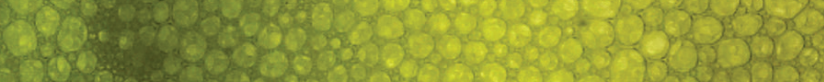

3098

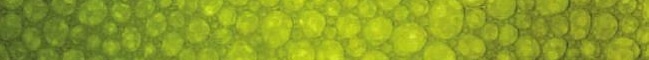

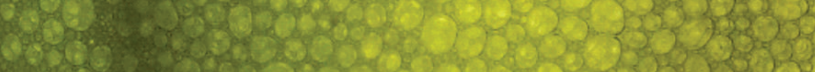
(10.0. 


\section{Chapter 1}

\section{Introduction}


Microalgal biomass is a promising source for food, feed, cosmetics and bulk chemicals and biofuels. Algal cells utilize carbon dioxide, light energy and inorganic nutrients for their reproduction which makes them an attractive and environmentally friendly resource. Compared to terrestrial plants, they do not require arable land or freshwater supply. Microalgae cultivation, however, is not yet efficient at large scale [1]. Traditional open raceway ponds offer an easy and economical cultivation, however, the cultivation conditions are not controlled, cultures might get contaminated, and only low biomass densities can be achieved $[2,3]$. In contrast, closed photobioreactors offer increased biomass productivities and elevated biomass concentrations because of better control of cultivation conditions and increased area to volume ratio. But these benefits come at a cost because closed photobioreactors require more capital expenses and often require more energy for operation [3]. The most common large-scale closed photobioreactor types are flat panel and tubular photobioreactors. Currently, large scale production is only feasible for high value products. For the production of bulk chemicals or commodities, the operation and installation costs of photobioreactors have to be reduced [1].

\subsection{Limitations of current production systems}

The greatest limitations of current large-scale photobioreactors lies in the low biomass density achieved $[4,5]$. The biomass density generally is below $3 \mathrm{~g} \mathrm{~L}^{-1}$, even during cultivation in closed photobioreactors [6]. Low density algal suspensions are difficult to harvest due to the small size of the algal cells, and the similar density of the cells and growth medium [7]. Several methods have been developed for harvesting of the algal cells. These methods include centrifugation, sedimentation, flocculation, flotation and filtration. These technologies are specific for algal strains and biomass concentrations. Generally, a combination of these methods is applied; biomass thickening followed by a dewatering step to achieve a concentrated biomass paste [8]. Harvesting accounts for $20-50 \%$ of the algal biomass costs [7]. In addition to the high harvesting costs, low biomass concentrations also involve processing of large liquid volumes, increasing the costs for pumping, mixing, and water intake and discharge.

A photosynthetically growing algal culture requires carbon dioxide $\left(\mathrm{CO}_{2}\right)$, and oxygen $\left(\mathrm{O}_{2}\right)$ needs to be removed from the photobioreactor. Both $\mathrm{O}_{2}$ and $\mathrm{CO}_{2}$ transfer require gassing and mixing and, as such, lead to high operational energy requirements [9, 10]. Tubular photobioreactors have poor mass transfer characteristics [3] and the accumulation of $\mathrm{O}_{2}$ might become a problem [3]. Flat panel photobioreactors can achieve better mass transfer capacity at the expense of high aeration rates [10]. Reduced aeration rates could account for $50 \%$ reduction 
in energy use for biomass production [6]. Therefore, increased $\mathrm{CO}_{2}$ and $\mathrm{O}_{2}$ transfer rates would contribute to more efficient photobioreactors.

\subsection{Concepts for improved photobioreactor design}

In order to produce biomass at high densities, photobioreactors with reduced light path are required. A short light path can be translated to a high illuminated surface to volume ratio (SVR), which also contributes to reduced dark volume fractions caused by microalgal selfshading. Dark zones in a photobioreactor will lower biomass productivity because of respiration (i.e. cellular maintenance), and handling this extra liquid volume represents energy costs. An alternative way to achieve a high SVR and eliminate light gradients in photobioreactors is to apply internal illumination [11]. A high SVR can also lead to light dilution, since the light falling on the reactor can be distributed over a larger surface [9]. Reducing the irradiance by applying light dilution can prevent oversaturation of the microalgal photosystem and can protect algal cells for photoinhibition.

Foams are characterized with low liquid fractions because part of the reactor volume is filled with a gas phase. Therefore, foams can be beneficial as a growth matrix for algal cells for efficient biomass production, since they offer an increased SVR and a reduced light absorption path. In addition, foams have an increased inner surface between the gas bubbles and the surrounding liquid phase likely improving mass transfer of $\mathrm{CO}_{2}$ and $\mathrm{O}_{2}$. In foams, the gas phase is entrapped in the foam bubbles, preventing the rapid rise and escape of the gas bubbles from the liquid phase and thus increasing the gas residence time. Liquid foams are stabilised by surface active molecules. These surfactants reduce the surface tension of the liquid and thereby allow the formation of foams when gassing the surfactant containing liquid with small gas bubbles.

\subsection{Characterization of liquid foams}

Liquid foams consist of gas bubbles dispersed in a continuous liquid phase. In dry foams, defined as liquid fractions below $5 \%$, the gas bubbles have a polyhedral shape. Between two bubbles a thin film is present, and at the junction of three bubbles so-called Plateau borders are formed. The junction of four Plateau borders is called a node. The structure of dry foams is presented in Figure 1.1. Most liquid in these dry foams reside in the Plateau borders and nodes. 


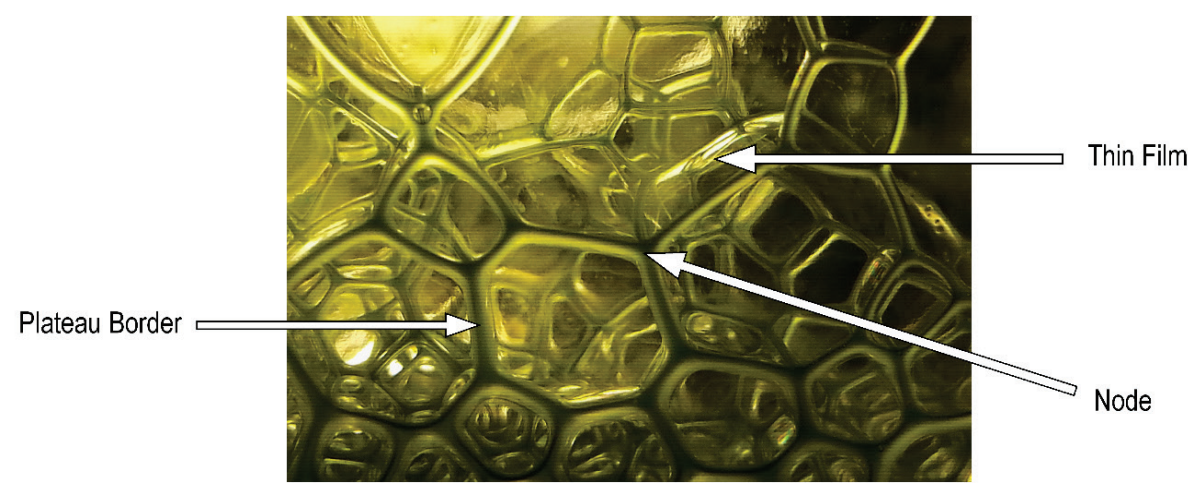

Figure 1.1. Structure of dry foams.

At high liquid fractions $(>15 \%)$, foams are referred as wet and the gas bubbles are nearly spherical. Above a certain liquid fraction, bubbles are not closely packed anymore and can move freely. This state is called bubbly liquid instead of foam. The maximal liquid fraction of foams before the bubbly liquid state is reached is $36 \%$ for monodisperse foam with homogenous bubble size, and $26 \%$ for polydisperse foam with a diverse bubble size distribution [12]. Foams can be formed by introducing gas into water containing surfactants, by e.g. shaking, mixing, gas sparging or via chemical/biological reactions [13]. Surfactant molecules will attach to the gas-liquid interfaces by orientating their hydrophilic head to the liquid phase and the hydrophobic tail to the gas phase. Foamability of a solution is influenced by the surface tension of the solution, the diffusion rate of surfactant molecules towards the interphase, and the absorption rate of the surfactant at the interfaces [14]. The thin films of foams are stabilized by steric, hydration, and hydrophobic repulsion forces. In case of ionic surfactants also electrostatic forces play a role [14].

Foam destabilization takes place as a result of liquid drainage due to gravity, coarsening, and coalescence. During coarsening, diffusional gas exchange takes place between adjacent bubbles because of pressure differences in the bubbles. Coarsening leads to a change in the bubble size distribution which also affects liquid drainage. Coalescence is the process where the thin liquid films between bubbles break leading to bubble growth and foam collapse. Next to these mechanisms, the drainage of foams and foam stability is also influenced by the addition of particles. Particle size, shape, and hydrophobicity are determining whether foam drainage is slowed down or accelerated [14]. Liquid drainage has a great influence on the liquid fraction profile of the foam: far away from the foaming solution, dry foam is present, while the foam is wet close to the interface where the foam is formed [12]. Figure 1.2 shows a picture of a foam 
under gravitational drainage where this liquid fraction gradient is observable. The white and opaque colour of foam in Figure 1.2 is due to light scattering on the multiple gas-liquid interfaces of the foams.

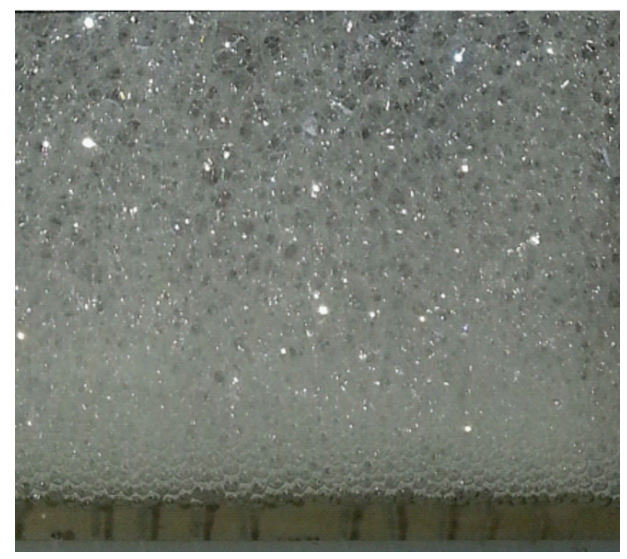

Figure 1.2. Picture of a rising foam column. The foam near the interface with the foaming solution is wet and composed of spherical bubbles, while at the top of the foam column dry foam with polyhedral bubbles are present.

\subsection{Overview of the application of foam-bed reactors}

Foam-bed reactors are known for the physical-chemical treatment of gases [15]. Although foam-beds are not yet applied in industry, continuous research is being conducted in this field. These reactor types are advantageous for contaminant removal of gas streams because of their increased interfacial area. Due to the large interfacial area, high mass transfer rates can be achieved, facilitating the absorption of compounds from the gas phase to the liquid phase. In the liquid phase, these compounds are absorbed and can be modified via chemical reactions. Next to large interfacial area, foam-beds are advantageous because they offer a low pressure drop and increased contact time between the gas and liquid phases [16-18]. Foam-beds can also be operated as bioreactors. Similarly to foam-beds for chemical treatments, bioactive foam reactors are also used for contaminant removal of gas streams. However, instead of chemical reaction of the absorbed pollutants, the microorganisms dispersed in the foam phase are responsible for the degradation [19]. The degrading microorganisms are actively growing at high cell density in the liquid phase of the foams. When microorganisms are applied, a biocompatible surfactant is required. Also for these biological foam-beds the advantage relies on the increased surface area of the foam leading to high pollutant removal rates. The absorption of organic pollutants can be further facilitated by the introduction of an organic-phase dispersed in the liquid phase of the foam as an emulsion [20,21]. 


\subsection{The foam-bed photobioreactor concept}

A liquid foam-bed can also be applied for the cultivation of microalgal cells. In this thesis, the application of a foam-bed photobioreactor for microalgae cultivation is evaluated for the first time. This novel idea of a foam-bed photobioreactor provides an alternative cultivation system next to traditional reactors. In a liquid foam-bed photobioreactor, foams are formed by distributing $\mathrm{CO}_{2}$-enriched gas into a surfactant-containing microalgae solution. Due to the continuous gas supply, the foam level rises inside the reactor. Microalgal cells reside in the thin films, Plateau borders and nodes of the generated foams, while the $\mathrm{CO}_{2}$ enriched gas phase is entrapped within the gas bubbles. A picture of a rising, microalgae-enriched, foam column is presented in Figure 1.3. During photoautotrophic microalgal growth on light, simultaneous $\mathrm{CO}_{2}$ uptake and $\mathrm{O}_{2}$ production takes place. After a certain time period, the gas bubbles have to be broken and reformed because of $\mathrm{CO}_{2}$ depletion and $\mathrm{O}_{2}$ enrichment. Therefore, the rising foam has to be continuously broken at the top of the reactor. After foam breakage, the gas released from the collapsed bubbles is allowed to escape to the environment, while the microalgal suspension liberated from the broken foam is incorporated again in freshly formed foam.

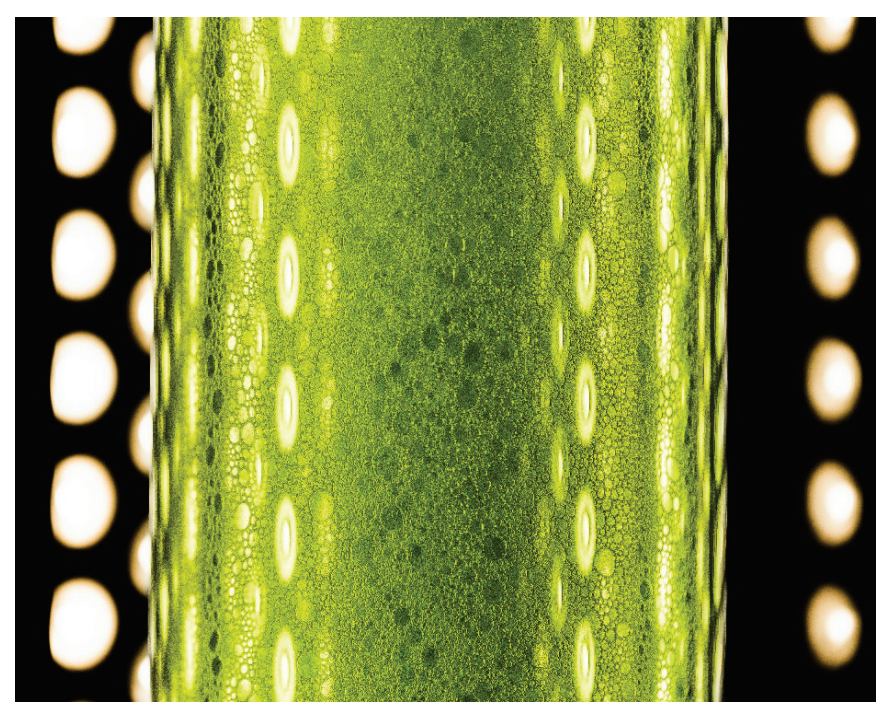

Figure 1.3. Picture of a bench-scale foam column containing microalgae illuminated by white LED lamps. 


\subsection{Advantages of the liquid foam-bed photobioreactor}

Foam-bed photobioreactors own several advantages over traditional, liquid phase based cultivation systems. Due to the low liquid fraction of foams, the light path over which light absorption takes place can be severely reduced in foam-beds. At the same irradiance, a reduction of the light absorption path enables cultivation at higher biomass densities. Flat panel reactors typically have an optical depth of a few centimetres. When such a flat panel reactor is filled with foam, the light absorption path can be as short as a few millimetres. Generally, the liquid fraction of the foam is around 5\%, which can theoretically result in a 20 times increase in biomass concentration [22]. This increase in biomass concentration allows for a dramatic reduction in the downstream processing costs. Additionally, the energy requirement for gas supply to the reactor will decrease because of the low hydrostatic pressure of the foam. This low pressure drop also contributes to reduced requirements for supporting material, which might lead to reduced construction costs of foam-bed reactor systems. Another benefit of the application of foam-bed photobioreactors lies in the improved gas transfer characteristics. The high transfer rate of $\mathrm{O}_{2}$ and $\mathrm{CO}_{2}$ between the gas and liquid phases is due to the high interfacial area of the foam-bed. Therefore, the gas supply rates can be severely reduced, leading to reduction in the operational costs of the reactor. Additionally, the $\mathrm{CO}_{2}$ uptake efficiency is increased in foam-beds because of the increased residence time of the gas phase inside the reactor.

\subsection{Aim and outline of this thesis}

The aim of this thesis was the development of a liquid foam-bed photobioreactor for microalgae cultivation. We aimed to maximize reactor productivity and process duration by improvements in reactor design, where we gave special attention to foam formation and foam break up. Additionally, our goal was to characterize the potential of this novel cultivation concept in a theoretical model, which can serve as a basis for further optimization and scale-up of foam-bed photobioreactors.

In Chapter 2 the proof-of-principle of the foam-bed photobioreactor concept is described. In that study, we determined whether microalgae cultivation in liquid foam-beds is possible. For the development of a practical and operative reactor design, foam formation and foam break up were studied in detail. In order to break the foam, different foam breaking methods were compared and evaluated. As a foam stabilizing agent, bovine serum albumin (BSA) was applied because it is a natural, non-toxic protein with good foaming capacity. Foam formation was 
optimized by testing different gas flow rates and surfactant concentrations. We demonstrated that algae grew rapidly in BSA stabilized foam but this protein is not suitable for long term algal cultivation due to its rapid degradation.

In Chapter 3, the selection of a suitable foam stabilizing agent that can enable long term foam formation is presented. Different surfactants have different stabilities and the type of surfactant determines the properties of the formed foams and the microalgae content of the foam. In addition, surfactants potentially can be toxic to microalgae and inhibit their growth. Therefore, we first determined the most important criteria that good foam stabilizing agents have to meet. Afterwards, different surfactants were compared according to these criteria, and the best surfactant was selected for the foam-bed photobioreactor.

In Chapter 4, we describe the application of the best surfactant selected in Chapter 3 for microalgae cultivation in an improved foam-bed photobioreactor. In order to optimize reactor operation, the foam-bed photobioreactor was completely re-designed. We characterized reactor performance in terms of mixing, light distribution, mass transfer, pressure drop and productivity. The improved lab-scale foam-bed photobioreactor enabled long-term microalgae cultivation at high biomass densities.

In Chapter 5 we present a model study on the potential of liquid foam-bed photobioreactors. We modelled the productivity of the reactor and determined the effect of different operational parameters on productivity and biomass specific operational energy requirements. The model gave detailed insight in the liquid fraction gradient, light distribution, and mass transfer in the liquid foam-bed. Additionally, the productivity and energy requirements of foam-bed photobioreactors could be evaluated and compared to traditional flat panel cultivation systems.

In Chapter 6 the achieved results are discussed and further improvements of reactor design are proposed. Additionally, the design of large scale, outdoor foam-bed photobioreactors is discussed. The most important issues related to scale-up, such as foam formation and break up, re-use of surfactants, reactor material choice and control strategies such as foam level and temperature control are evaluated in detail. In addition, our results are compared with the most recent developments in photobioreactor design. 


\section{References}

[1] F.G. Acién, J.M. Fernández, J.J. Magán, E. Molina, Production cost of a real microalgae production plant and strategies to reduce it, Biotechnol. Adv. 30 (2012) 1344-1353.

[2] O. Pulz, Photobioreactors: production systems for phototrophic microorganisms, Appl. Microbiol. Biotechnol. 57 (2001) 287-293.

[3] C.U. Ugwu, H. Aoyagi, H. Uchiyama, Photobioreactors for mass cultivation of algae, Bioresour. Technol. 99 (2008) 4021-4028.

[4] H. Qiang, Y. Zarmi, A. Richmond, Combined effects of light intensity, light-path and culture density on output rate of Spirulina platensis (Cyanobacteria), Eur. J. Phycol. 33 (1998) 165-171.

[5] N. Uduman, Y. Qi, M.K. Danquah, G.M. Forde, A. Hoadley, Dewatering of microalgal cultures: a major bottleneck to algae-based fuels, J. Renew. Sustain. Energy 2 (2010) 012701.

[6] J. Ruiz, G. Olivieri, J. de Vree, R. Bosma, P. Willems, H. Reith, M. Eppink, D.M. Kleinegris, R.H. Wijffels, M. Barbosa, Towards industrial products from microalgae, Energy Environ. Sci. 9 (2016) 3036-3043.

[7] J.J. Milledge, S. Heaven, A review of the harvesting of micro-algae for biofuel production, Rev. Environ. Sci. Bio/Technol. 12 (2013) 165-178.

[8] A.I. Barros, A.L. Gonçalves, M. Simões, J.C. Pires, Harvesting techniques applied to microalgae: a review, Renew. Sust. Energ. Rev. 41 (2015) 1489-1500.

[9] C. Posten, Design principles of photo-bioreactors for cultivation of microalgae, Eng. Life Sci. 9 (2009) 165-177. [10] N.-H. Norsker, M.J. Barbosa, M.H. Vermuë, R.H. Wijffels, Microalgal production - A close look at the economics, Biotechnol. Adv. 29 (2011) 24-27.

[11] A. Jacobi, E. Bucharsky, K. Schell, P. Habisreuther, R. Oberacker, M. Hoffmann, N. Zarzalis, C. Posten, The application of transparent glass sponge for improvement of light distribution in photobioreactors, J. Bioprocess Biotech. 2 (2012) 1000113.

[12] W. Drenckhan, S. Hutzler, Structure and energy of liquid foams, Adv. Colloid Interface Sci. 224 (2015) 1-16.

[13] W. Drenckhan, A. Saint-Jalmes, The science of foaming, Adv. Colloid Interface Sci. 222 (2015) 228-259.

[14] J. Wang, A.V. Nguyen, S. Farrokhpay, A critical review of the growth, drainage and collapse of foams, Adv. Colloid Interface Sci. 228 (2016) 55-70.

[15] J. Biswas, R. Kumar, Mass transfer with chemical reaction in a foam bed contactor, Chem. Eng. Sci. 36 (1981) 1547-1556.

[16] P.S. Shah, R. Mahalingam, Mass transfer with chemical reaction in liquid foam reactors, AIChE J. 30 (1984) 924-934.

[17] A.A. Gaikwad, A.N. Bhaskarwar, Absorption of pure carbon-dioxide gas in a foam-bed reactor, Proceedings of European Congress of Chemical Engineering (ECCE-6), Copenhagen, 2007.

[18] A.A. Gaikwad, N. Challapalli, A.N. Bhaskarwar, Carbonation of Barium Sulfide in a Foam-Bed Reactor, Chem. Eng. Commun. 197 (2010) 804-829

[19] J. Song, Y. Kim, Y. Son, J. Khim, A bioactive foam reactor for the removal of volatile organic compounds: system performance and model development, Bioprocess Biosyst. Eng. 30 (2007) 439-446.

[20] E. Kan, M.A. Deshusses, Development of foamed emulsion bioreactor for air pollution control, Biotechnol. Bioeng. 84 (2003) 240-244.

[21] F.G. Shahna, F. Golbabaei, J. Hamedi, H. Mahjub, H.R. Darabi, S.J. Shahtaheri, Treatment of Benzene, Toluene and Xylene Contaminated Air in a Bioactive Foam Emulsion Reactor, Chin. J. Chem. Eng. 18 (2010) 113-121.

[22] W. Blanken, P.R. Postma, L. de Winter, R.H. Wijffels, M. Janssen, Predicting microalgae growth, Algal Res. 14 (2016) 28-38. 


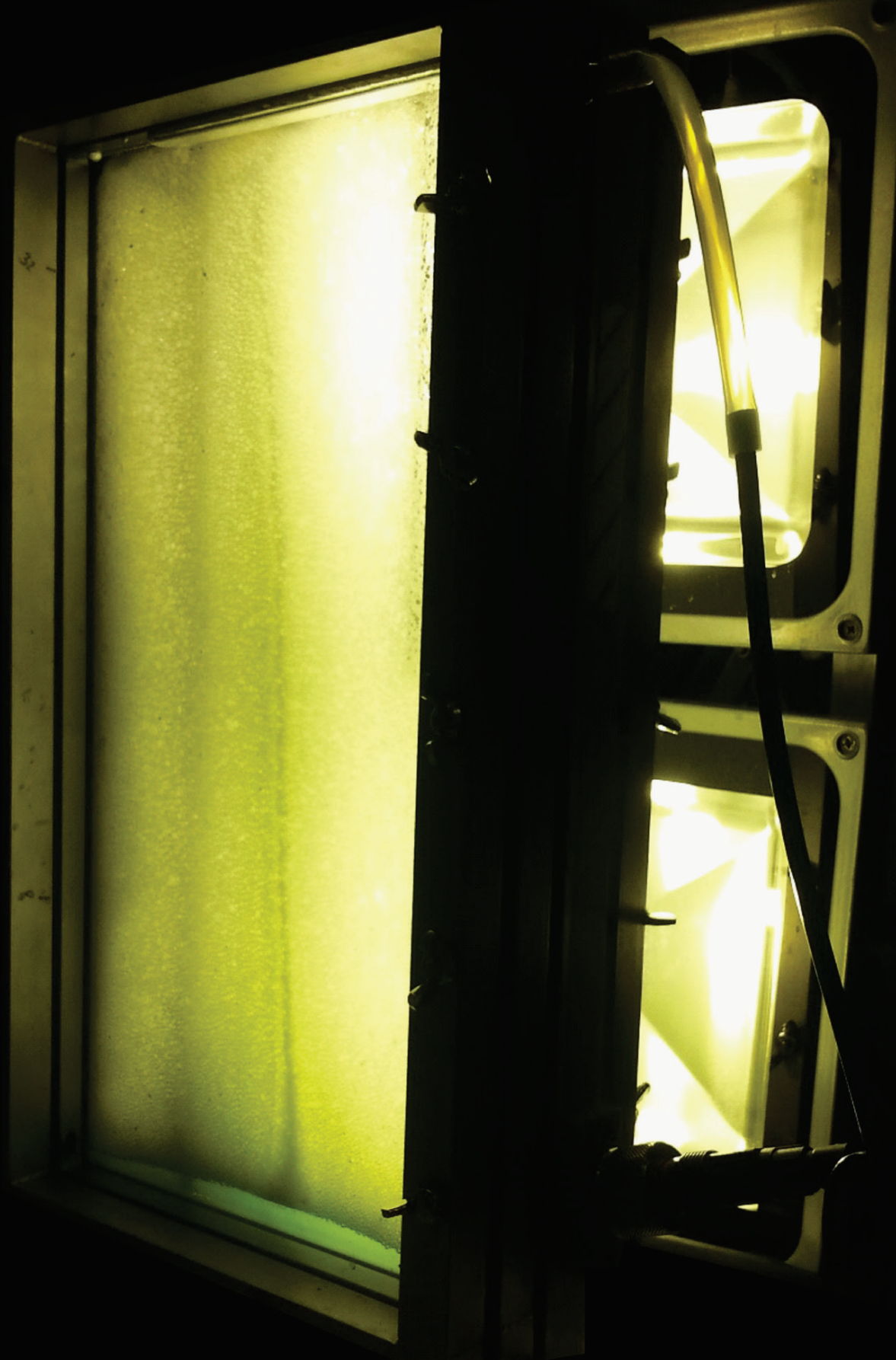




\title{
Chapter 2
}

\section{A liquid foam-bed photobioreactor for microalgae}

\author{
production
}

This chapter is published as:

A. Janoska, P.P. Lamers, A. Hamhuis, Y. van Eimeren, R.H. Wijffels, M. Janssen, A liquid foam-bed photobioreactor for microalgae production, Chem. Eng. J. 313 (2017) 1206-1214. 


\begin{abstract}
A novel concept of cultivating microalgae in liquid foam was developed with the intention of reducing biomass production costs. This cost reduction is based on reduced harvesting costs due to high biomass densities, and reduced energy requirements due to improved mass transfer and lower pressure drop in the foam-bed photobioreactor. Foam generation could be controlled by adding foaming agents and employing homogenous gas distribution at the bottom of the photobioreactor. In order to refresh the gas phase entrapped in the bubbles, and ensure sufficient $\mathrm{CO}_{2}$ for microalgal growth, different foam break-up methods were evaluated. A packed bed filled with large hydrophobic beads resulted in efficient foam break-up at minimal pressure drop. It was shown that microalgae (Chlorella sorokiniana) can grow in the liquid channels of liquid foams stabilised by the protein Bovine Serum Albumin, and that the culture can withstand the physical processes of foam formation and foam break-up. An average growth rate of 0.10 $\mathrm{h}^{-1}$ was observed. The quantum yield of photosystem II photochemistry remained maximal during the reactor runs, indicating that photosynthesis was not impaired. The results obtained show that cultivation of microalgae in liquid foams is a promising new concept.
\end{abstract}




\subsection{Introduction}

The production of useful substances of algal origin, including specialities for food and aquaculture as well as biofuels and bulk chemicals, requires energy-efficient and economically profitable cultivation systems [1-3]. Many studies highlighted the importance of photobioreactor design and operation as major factors influencing production costs [4-7]. The goal of this study is therefore the development of a novel microalgae cultivation system that could enable economically feasible microalgae cultivation by reducing biomass production costs. The major factors that determine the practical application of photobioreactors is rapid and energy-efficient transfer of carbon dioxide and oxygen [8], the dewatering of the harvested, dilute microalgal cultures [9], and the high energy input for aeration [10]. In this study a foambed photobioreactor with high gas holdup was developed since increased gas holdup results in both increased mass transfer and lower pressure drop. In addition, the foam-bed photobioreactor supports increased biomass concentration due to the thin liquid layers between the foam bubbles reducing microalgal self-shading. The concept of growing microalgae in liquid foam-bed photobioreactors is an innovative idea in the field of microalgae cultivation [11].

In a foam-bed reactor small gas bubbles are passed through a thin liquid layer resulting in foam generation. The liquid is either self-foaming or contains a foam stabilising agent. Thus, the culture is composed of a thin liquid layer at the bottom of the reactor with a large volume of foam exposed to (sun)light, above it. Due to the continuous gas supply, the generated foam bubbles rise. Simultaneously, the liquid film separating adjacent gas bubbles is continuously draining downwards due to gravity.

This novel concept has several potential advantages over traditional cultivation systems. First, when adopting flat-panel photobioreactors in combination with a liquid foam-bed the light path in the liquid film in the foam over which light absorption takes place is in the order of a few millimetres only. Consequently, the biomass concentration can be increased with an order of magnitude ( $\geq 10 \mathrm{~g} \mathrm{~L}^{-1}$ ) compared to liquid-filled flat-panel reactors, thereby reducing downstream processing costs with the same factor. Furthermore, a foam-bed reactor only contains a limited water volume (about $5 \% \mathrm{v} / \mathrm{v}$ ) resulting in a low pressure drop relative to the height of the photobioreactors. Therefore, the concept might enable energy reduction on gassing due to the low pressure drop present in the reactor. Besides, due to the low pressure drop in the reactor, the carrier capacity of the structure supporting the photobioreactors can be reduced considerably, thereby lowering construction costs of large-scale systems. Also, the high 
interfacial area created between the gas and water with microalgae contributes to the reduced energy requirement of the foam-bed reactor. The high interfacial area results in a high transfer capacity for both oxygen and carbon dioxide. Finally, the residence time of the gas in the photobioreactor is increased by orders of magnitude since the gas is entrained within the liquid films of the foam. This leads to a much more efficient use of carbon dioxide.

Foam-bed reactors for chemical-physical treatment of gases are known. Owing to the enhanced mass transfer capacity and low pressure drop of these systems, efficient contaminant removal of gas streams is possible. In these reactor systems, components of the gas move from the gas bubbles to the thin liquid films, followed by a chemical reaction in the liquid phase of the foam [12-14]. Foam-bed reactors are also used as bioreactors for contaminant removal from gas streams [15-20]. In these systems the pollutant-degrading microorganisms are grown in the thin liquid films in the foams. The performance of foamed emulsion bioreactors (a type of foambed bioreactor, where organic phase emulsion and pollutant-degrading microorganisms are foamed and the resulting gas bubbles contain the pollutant) exceed the performance of any other reactor system for air pollutant control [16]. These reactors rely on high density cultivation of microorganisms in order to reach high removal rates, increased gas-liquid interfacial area provided by the foams, and elimination of clogging problems compared to immobilized beds [16].

For the design of a foam-bed reactor, foam formation and foam break-up are fundamental. The properties of the formed foams are dependent on the gas distributer design, as it influences the bubble size of the foam. More specifically, if the gas distributor creates smaller bubbles, more stable and wet foam will be formed [21]. In contrast, larger bubbles will rise faster to the surface and collapse more rapidly [22]. Besides gas distribution, also the gas flow rate and surfactant concentrations play key roles in determining the foam properties.

In order to support maximal microalgae production in a foam-bed photobioreactor, the $\mathrm{CO}_{2}$ supply must be sufficient. For this reason, the foam bubbles have to be broken in order to refresh the entrapped gas. Ideally, a foam bubble ruptures just before the carbon dioxide is depleted, and/or oxygen builds up to inhibiting levels. For inducing foam break-up, various methods have been reported in literature. The simplest method is spontaneous, self-break-up of the foam [18]. This method is based on natural destabilisation mechanisms, including foam drainage, coalescence, and coarsening. Liquid drainage from the foam is caused by gravity and causes thinning of the liquid films between bubbles. This thinning can lead to film rupture, resulting 
in coalescence of the neighbouring bubbles. Coarsening takes place due to gas diffusion from the small bubbles to the larger ones, due to the pressure difference inside them. All these processes can result in bubble growth and eventually to foam destabilisation [23].

Another, commonly used method is the use of chemical antifoams or defoamers [12, 24-27]. These methods are efficient in destroying and controlling foams, but in several cases they cannot be used. For instance, the antifoaming agents can adsorb to cell surfaces and consequently inhibit growth of the microorganism, they can cause contamination, reduce mass transfer, and exhibit adverse effect on downstream processing of the product (e.g. separation, purification) [28-30]. Foam breaking by mechanical means is free of such problems, however, substantial power is required for the operation of the devices [30]. Mechanical methods are mainly based on shear forces [28], or on centrifugal forces [31], and they include spraying liquid on the foam [16, 20, 32] or breaking the foam by rotating parts [21, 29, 33]. Mechanical and ultrasonic vibrations are also often used [28]. Compared to chemical or mechanical foam breaking methods, a foam eliminating net [34] can reduce the operational costs and the contamination of the media can be prevented. Together, these studies highlight the variety of possibilities for foam break-up, which is a crucial factors in establishing and further improving foam-bed reactor systems.

This study aims to develop a liquid foam-bed photobioreactor for microalgal growth with continuous foam formation and foam break-up. For that, optimal foam formation settings were experimentally defined and also an efficient foam break-up method was developed. Furthermore, the possibility of microalgal growth in protein stabilised foams was evaluated. In order to assess whether microalgae are able to withstand the shear stresses involved in foam formation and break-up, the biomass concentration and the quantum yield of photosystem II photosystem were monitored.

\subsection{Materials and Methods}

\subsubsection{Experimental set-up}

The experimental set-up consisted of a foam-bed photobioreactor, a foam breaker column and a recirculation pump (Figure 2.1). The foam-bed photobioreactor itself consisted of a flat panel reactor chamber and an adjacent water chamber for temperature control. The reactor had a height of $40 \mathrm{~cm}$, and a width of $20 \mathrm{~cm}$. The reactor had a depth of $2.7 \mathrm{~cm}$ and the reactor volume was approximately $2.2 \mathrm{~L}$. The reactor had round edges on the top in order to avoid foam to 
accumulate and remain there. The reactor plates were made of glass and the reactor fame was made of polyether ether ketone (PEEK). The glass plates were treated with a solution of concentrated sulfuric acid (98 wt.\%) and hydrogen peroxide solution (30 wt.\%) in a 3:1 ratio. This solution cleaned the glass surface and rendered it hydrophilic; a contact angle of $12^{\circ}$ was reported [35]. The cleaned glass plates were washed with distilled water. The contact of the foam with hydrophilic walls, as opposed to hydrophobic surfaces, had a positive effect on foam stability inside the reactor, enabling faster foam rise and reducing the extent of coalescence at the walls.

The inlet gas was composed of $5.5 \mathrm{v} / \mathrm{v} \%$ carbon dioxide in nitrogen gas and was supplied with a total flow rate of $614 \mathrm{NmL} \mathrm{min}^{-1}$ by mass flow controllers (Brooks Instrument B.V. Model 5850S). This gas was filtered with $0.2 \mu \mathrm{m}$ filters (Whatman Polyvent 500) prior to entering the reactor. The filtered gas was distributed through a stainless steel gas distributor with small conical holes $(30 \mu \mathrm{m}$ and $100 \mu \mathrm{m}$ hole diameter on the top and the bottom of the cone, respectively). The gas distributing plate was placed on the bottom of the reactor, enabling bubble formation over $40 \%$ of the cross sectional area in order to ensure good mixing and avoid microalgae settling. The gas distributor created a large number of small, homogenous bubbles stimulating continuous foam formation. The foam rising to the top of the reactor was allowed to leave through three outlets $(0.9 \mathrm{~cm}$ diameter) and was led to the foam breaker device via silicone tubing with $0.8 \mathrm{~cm}$ diameter. These three separate outlets $(2$ at the sides and one in the middle) were required in order to avoid foam to accumulate and remain in the reactor.

As a foam breaker device, a packed bed column filled with hydrophobic beads was established. The internal diameter of the glass column was $5 \mathrm{~cm}$ and it had a volume of $216 \mathrm{~mL}$. The glass surface was rendered hydrophobic by applying a coating called Sigmacote (Sigma-Aldrich). The hydrophobic beads had an average diameter of $6.3 \mathrm{~mm}$ and were made of polytetrafluoroethylene (PTFE) produced by FTL Technology, and they were mixed with polydimethylsiloxane (PDMS) cubes of approximately the same size. The PDMS cubes were fabricated from a Silicone Elastomer Kit (Sylgard 184 Silicone Elastomer and curing agent, Dow Corning) and the ratio of the base monomers and the curing agent was 10:1. After mixing and eliminating the gas bubbles, the mix was poured in a glass petri dish. The PDMS was cured for 1 hour in the oven at $80^{\circ} \mathrm{C}$. After cooling overnight, the PDMS sheet was removed from the petri dishes and placed in the oven at $60^{\circ} \mathrm{C}$ for overnight. This PDMS slab was afterwards cut to small pieces with a sharp knife. 
After the foam entered from the top into the foam breaker, it was led through the packed bed column and subsequently ended up in a vessel for gas-liquid separation, as depicted in Figure 2.1. The liquid was pumped back to the reactor by a peristaltic pump. The liquid volume in the photobioreactor was controlled by avoiding evaporation or condensation inside the reactor. The nitrogen gas was humidified by leading it through a $4 \mathrm{~mm}$ inner diameter tubing to the bottom of a $500 \mathrm{~mL}$ water bottle kept at $2^{\circ} \mathrm{C}$. Dry $\mathrm{CO}_{2}$ gas was mixed with the humidified $\mathrm{N}_{2}$ gas before entering the reactor. The gas left the reactor through a condenser maintained at $2^{\circ} \mathrm{C}$.

A pH and temperature sensor were incorporated in the reactor. The temperature sensor was placed at the top of the reactor, measuring the temperature of the upper third part of the foam. The $\mathrm{pH}$ was measured within the foam-bed at the bottom just above the bulk liquid level. The $\mathrm{pH}$ was not controlled but it remained $6.7 \pm 0.3$ throughout the experiments. The culture temperature was maintained at $37{ }^{\circ} \mathrm{C}$ by controlling the temperature in the water jacket by recirculating the water through a water bath. The reactor was illuminated from one side by two warm-white LED floodlights with a 45 mil Bridgelux LED chip, stacked on top of each other providing an intensity of $334 \pm 16 \mu \mathrm{mol}$ PAR photons $\mathrm{m}^{-2} \mathrm{~s}^{-1}$ across the reactor surface. Pictures of the foam-bed photobioreactor are presented in Figure 2.2. 


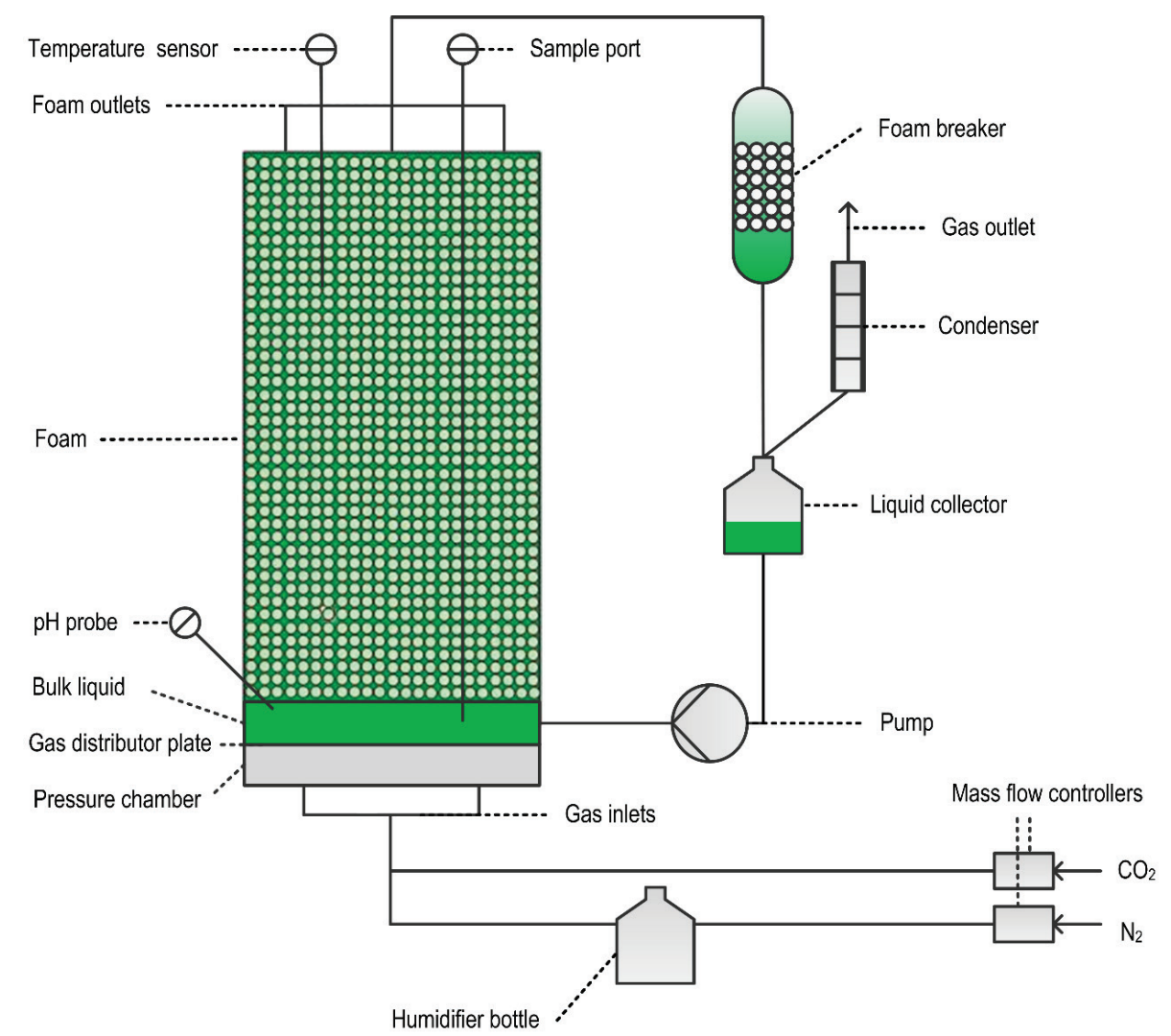

Figure 2.1. Schematic overview of the foam-bed photobioreactor. Gas is fed at the bottom of the reactor via a gas distributor plate, releasing fine gas bubbles into the bulk liquid, continuously creating foam. The foam leaves the reactor on the top and is then transported towards the foam breaker. After break-up of the foam the separated liquid and gas phase end up in a collector vessel from where the gas leaves through the condenser and the liquid is pumped back into the reactor. 


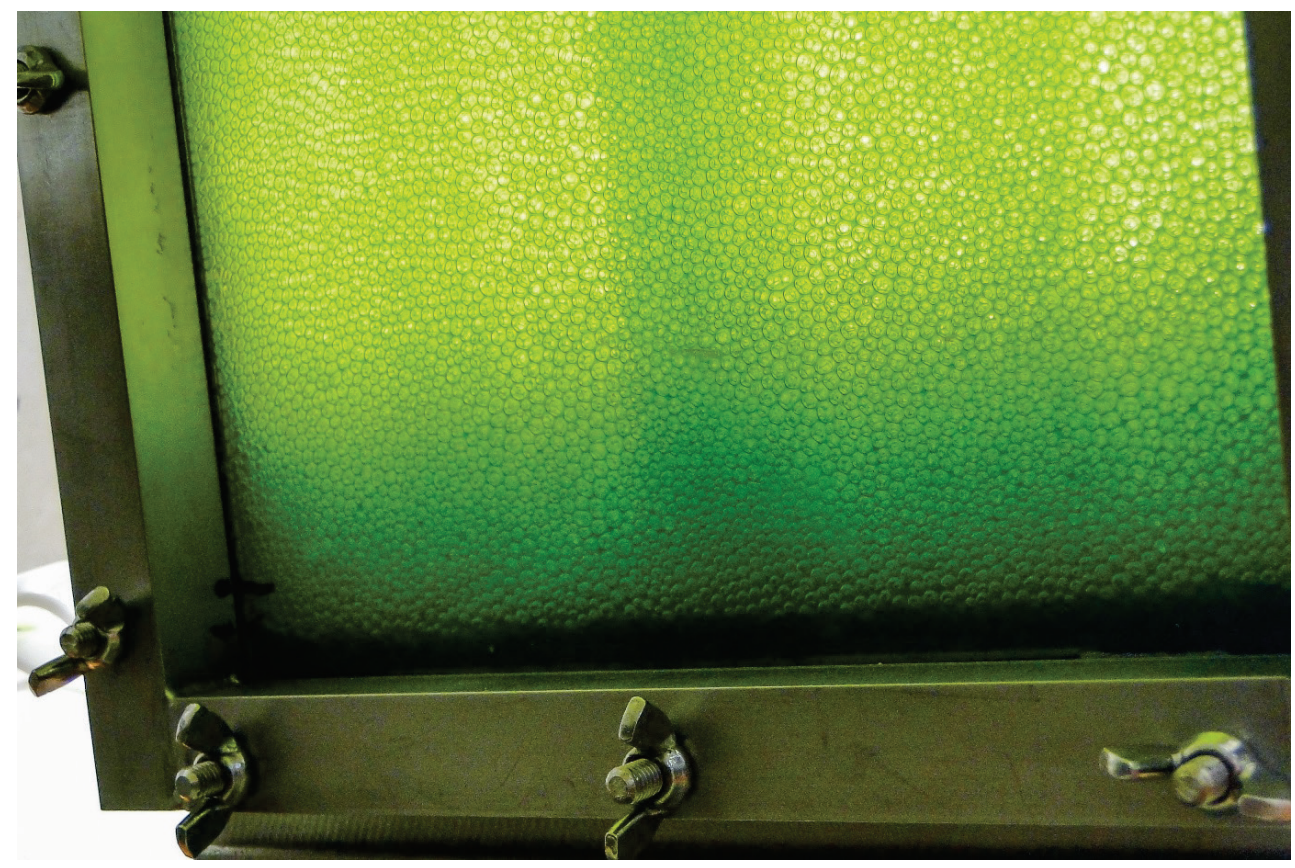

Figure 2.2. Picture of the foam in the foam-bed reactor containing microalgae.

\subsubsection{Microalga and growth medium}

Chlorella sorokiniana strain CCAP 221/8K was obtained from the Culture Collection of Algae and Protozoa (CCAP, Oban, Scotland). C. sorokiniana was grown in shake flasks, on a media based on 3 times concentrated M8a media [36], containing the following nutrients: urea $60 \mathrm{mM}$, $\mathrm{KH}_{2} \mathrm{PO}_{4} 7.88 \mathrm{mM}, \mathrm{Na}_{2} \mathrm{HPO}_{4} .2 \mathrm{H}_{2} \mathrm{O} 2.12 \mathrm{mM}, \mathrm{MgSO}_{4} .7 \mathrm{H}_{2} \mathrm{O} 4.87 \mathrm{mM}, \mathrm{CaCl}_{2} .2 \mathrm{H}_{2} \mathrm{O} 0.26 \mathrm{mM}$, EDTA ferric sodium salt $948 \mu \mathrm{M}, \mathrm{Na}_{2}$ EDTA. $2 \mathrm{H}_{2} \mathrm{O} 300 \mu \mathrm{M}, \mathrm{H}_{3} \mathrm{BO}_{3} 3 \mu \mathrm{M}, \mathrm{MnCl}_{2} .4 \mathrm{H}_{2} \mathrm{O} 196.76$ $\mu \mathrm{M}, \mathrm{ZnSO}_{4} .7 \mathrm{H}_{2} \mathrm{O} 33.39 \mu \mathrm{M}, \mathrm{CuSO}_{4} .5 \mathrm{H}_{2} \mathrm{O} 21.99 \mu \mathrm{M}$. After the addition of all nutrients, the $\mathrm{pH}$ was adjusted with $\mathrm{NaOH}$ to $\mathrm{pH}$ 6.7. The same medium supplemented with $5 \mathrm{mM} \mathrm{NaHCO}_{3}$, (after setting of the $\mathrm{pH}$ ), was used for both the pre-cultivation and the foam-bed photobioreactor experiments.

The inoculum for the foam-bed photobioreactor was pre-grown in an airlift flat-panel photobioreactor, as described by de Mooij et al. [37]. This reactor was operated continuously with a dilution rate of $0.106 \mathrm{~h}^{-1}$. The $\mathrm{pH}$ was controlled at 6.7 by $\mathrm{CO}_{2}$ addition. The incident light intensity was $1400 \mu \mathrm{mol}$ photons $\mathrm{m}^{-2} \mathrm{~s}^{-1}$. This ensured an exponentially growing algal culture acclimated to high light intensities, thereby avoiding a lag phase during growth in the foam-bed photobioreactor. To inoculate this photobioreactor, cultures cultivated in shake flasks 
were used. These shake flasks were kept in an incubator containing $4 \% \mathrm{CO}_{2}$, operated at $37^{\circ} \mathrm{C}$, $454 \mu \mathrm{mol}$ photons $\mathrm{m}^{-2} \mathrm{~s}^{-1}$ and $120 \mathrm{rpm}$.

\subsubsection{Growth experiments foam-bed photobioreactor}

Prior to the growth experiments, the reactor was autoclaved to ensure sterile operation. The reactor was started with $150 \mathrm{~mL}$ of culture medium containing $1.75 \mathrm{~g} \mathrm{~L}^{-1}$ bovine serum albumin (BSA, Sigma-Aldrich) as a protein-based foaming agent. This medium was inoculated with Chlorella sorokiniana cultures to an optical density $(750 \mathrm{~nm})$ of $5.6 \pm 0.7$ units (equivalent to $2.1 \pm 0.2$ gram dry biomass per litre). After starting the gas supply to the reactor, the initial liquid present separated into two different segments: the wet foam phase and the remaining liquid layer on the bottom of the reactor above the gas distributing plate, referred to as the bulk liquid. The bulk liquid area was covered with aluminium foil to shade off light in order to avoid growth in that segment. Samples ( 2 to $3 \mathrm{~mL}$ ) were taken each 2 hours from the reactor bulk liquid and were analysed for algae concentration (optical density, cell number, and cell volume concentration), the maximum quantum yield of PSII photochemistry $\left(\mathrm{F}_{\mathrm{v}} / \mathrm{F}_{\mathrm{m}}\right)$, and the protein concentration. The temperature and $\mathrm{pH}$ inside the reactor were continuously monitored. The temperature and relative humidity of the outgoing gas was analysed at two hour intervals. The relative humidity of the ingoing gas was measured before the experiments using a humidity meter (H1, Testo Inc.). The water level and temperature of the humidifying bottle were kept constant, thus the initially measured humidity was representative for all the experiments.

\subsubsection{Analytical methods}

The microalgae concentration in the samples was determined by two different methods. Firstly, spectrophotometric analysis (DR 600 spectrophotometer from Hach Lange) was carried out. The measuring wavelengths employed were $680 \mathrm{~nm}$ and $750 \mathrm{~nm}$. The second method used was measuring cell number and cell volume concentration with a Beckman Coulter Multisizer 3 employing a $50 \mu \mathrm{m}$ aperture tube. The cell size distribution was determined in terms of cell volumes, from which the total cell volume concentration was calculated.

The BSA protein concentration in solution was determined by the Lowry method. Prior to analysis, samples were centrifuged at 20,000 RCF for 10 minutes to remove algae and bacteria from the sample. Supernatants were stored at $-24^{\circ} \mathrm{C}$ until analysis. These samples were diluted to a protein concentration less than $1.4 \mathrm{~g} \mathrm{~L}^{-1}$. Afterwards the Bio-Rad Dc protein assay kit was used for the analysis. The absorbance was determined by a measurement at $750 \mathrm{~nm}$ using the 
Tecan M200 Plate Reader, and a calibration curve made with BSA was used to convert the absorbance values to concentrations expressed in $\mathrm{g} \mathrm{L}^{-1}$.

Maximum photosystem II quantum yield was measured based on chlorophyll fluorescence with the AquaPen-C AP-C 100 fluorimeter (PSI, Czech Republic) [38]. Samples were diluted to an optical density (OD) at $750 \mathrm{~nm}$ of approximately 0.1 unit. The minimal fluorescence level was measured after 15 minutes incubation in the dark at a light intensity of $0.03 \mu \mathrm{mol}$ photons $\mathrm{m}^{-2}$ $\mathrm{s}^{-1}$ at $455 \mathrm{~nm}$. The maximal fluorescence was measured after a light pulse of $3000 \mu \mathrm{mol}$ photons $\mathrm{m}^{-2} \mathrm{~s}^{-1}$. The maximum photosystem II quantum yield $\left(\mathrm{F}_{\mathrm{v}} / \mathrm{F}_{\mathrm{m}}\right)$ is calculated as the difference of the maximal fluorescence of the sample $\left(\mathrm{F}_{\mathrm{m}}\right)$ and the minimal fluorescence $\left(\mathrm{F}_{0}\right)$, divided by the maximal fluorescence, resulting in $\left(\mathrm{F}_{\mathrm{m}}-\mathrm{F}_{0}\right) / \mathrm{F}_{\mathrm{m}}$.

Properties of BSA-stabilized foam were analysed by an automated foam analyser (Foamscan, Teclis- IT Concept, Logessaigne, France), adapted from Lech et al. [39]. Firstly, foam is generated by blowing nitrogen gas through a metal frit with small conical holes ( $30 \mu \mathrm{m}$ and 100 $\mu \mathrm{m}$ hole diameter on the top and the bottom of the cone, respectively) to a glass cylinder containing $60 \mathrm{~mL}$ of surfactant solution. After the foam volume has reached $400 \mathrm{~cm}^{3}$, the gas flow automatically stops. The liquid volume of the solution remaining on the bottom of the cylinder was monitored by conductimetry. The amount of liquid incorporated in the foam was calculated as the difference between the initial liquid volume and the liquid volume at the different time points. The volume percentage of liquid within the foam will be further referred to as the liquid holdup of the foam. The maximal liquid holdup represents the liquid holdup of the foam at the moment when the foam has reached its desired height and the gas distribution has been terminated. The foam volume was followed in time by a camera and consequent image analysis. Foam stability was measured in terms of the time needed until half of the foam volume had collapsed, and will be further referred to as the foam half-life $\left(t_{1 / 2}\right)$. The evolution of the bubble sizes was monitored by image analysis. Pictures were taken each 30 seconds after the gas flow had stopped, at a height of $8 \mathrm{~cm}$ above the gas distributor. The bubble size was calculated by image analysis software (Foamscan), from the first picture of the static foam. The temperature of the glass cylinder was kept at $37 \pm 2{ }^{\circ} \mathrm{C}$ in all experiments and controlled by a water bath. The gas flow rate for the experiments with different surfactant concentrations was $400 \mathrm{~cm}^{3} \mathrm{~min}^{-1}$, resulting in $2.4 \mathrm{~mm} \mathrm{~s}^{-1}$ superficial gas velocity. The BSA concentration for the experiments with different gas flow rates were $0.5 \mathrm{~g} \mathrm{~L}^{-1}$. The experiments were performed in duplicate. 


\subsection{Results and Discussion}

\subsubsection{System design}

In this section the most important aspect of the design of the foam-bed photobioreactor are presented.

\subsubsection{Optimization of foam formation}

In this study, Bovine Serum Albumin (BSA) was selected as foam stabilising agent. This surfactant is biocompatible [40, 41] and has a good foaming ability [42]. Firstly, the properties of foams formed by different BSA concentrations and different gas flow rates were determined. The observed relations were then applied to select optimal conditions for the operation of the foam-bed photobioreactor. This optimization aimed to create a wet and homogeneous foam in the reactor, which could be easily destabilized in the foam-breaker.

Initially, protein foams were analysed with an automated foam analyser (Foamscan). Foam stability in terms of foam volume half-life, liquid holdup of the foam, and bubble size were analysed as a function of BSA concentration and applied gas flow rates. In the range investigated, the protein concentration has more impact on bubble size, liquid holdup and foam stability compared to the gas flow rate. The results show that higher BSA concentrations result in smaller bubble sizes and more stable foams with higher liquid holdup (Figure 2.3 A, B, and C). These results are in agreement with other studies, and the underlying mechanism is described as follows. Increasing the surfactant concentration results in a lower surface tension, which in turn leads to a smaller bubble size, resulting in a wetter and more stable foam [21, 28]. Higher superficial gas velocities have similar effect: at increased gas velocities, slightly smaller bubbles are formed, resulting in increased foam stability and liquid holdup (Figure 2.3 D, E and F). At higher gas flow rates, the foam liquid holdup is increased due to the elevated upward liquid flux [43]. At low gas flow rates the time to reach a given volume is increased, which results in increased foam destabilisation. The longer time period for foam formation contributes to the decreased liquid holdup [44] and possibly this also adds to the decreased foam stability occurring at reduced gas flow rates. A closer look on the graphs presented in Figure 2.3 show that a decreasing bubble size goes together with a more stable and wet foam. At increased gas flow rates and at increased surfactant concentrations the foam appeared more homogenous, as also expressed by the standard deviations of the average bubble sizes (Figure 2.3 C and F). 

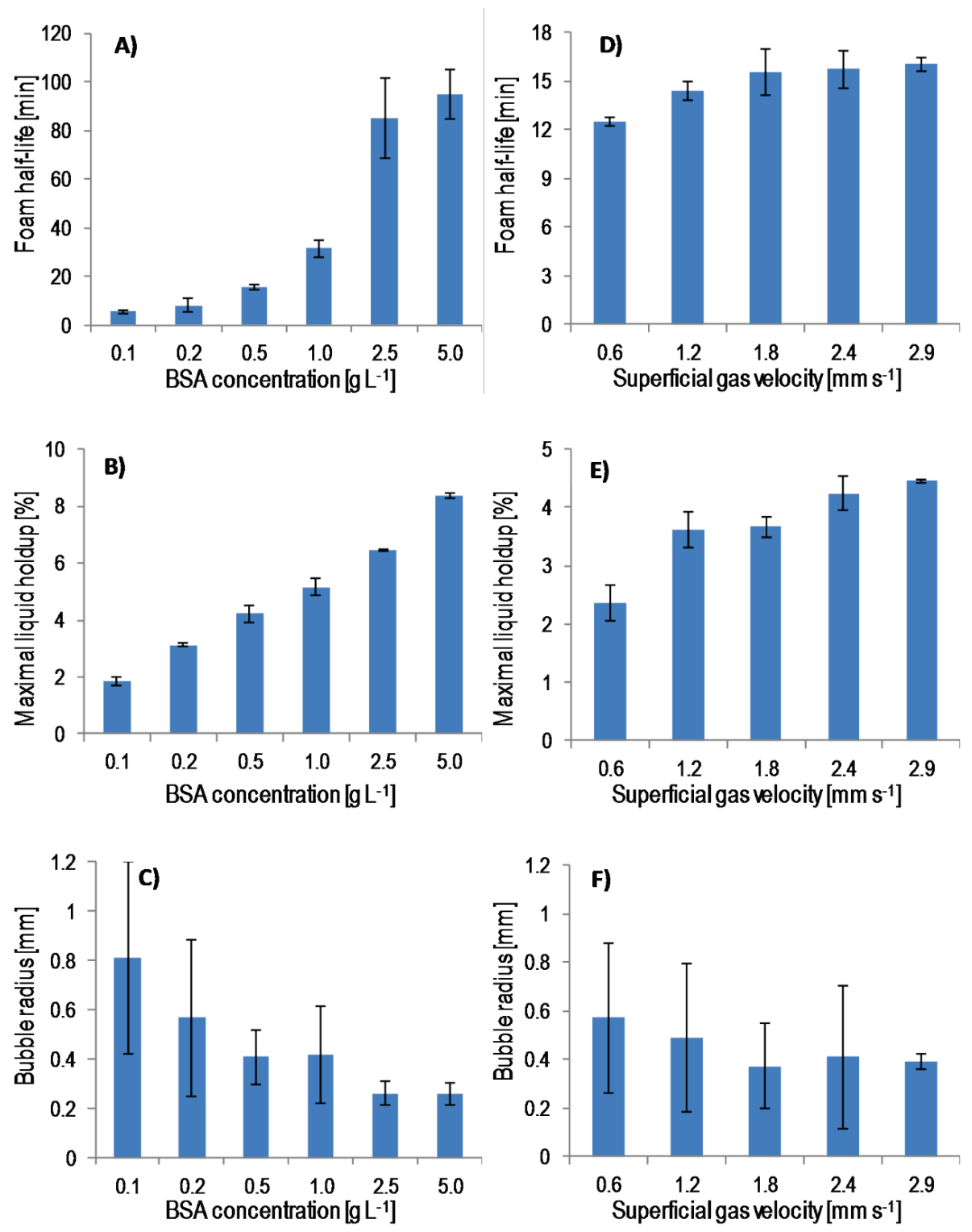

Figure 2.3. Effect of BSA concentration on the half-life of the foam (A), the maximal liquid holdup of the foam (B), and the average bubble radius of the foam (C). Effect of superficial gas velocity at $0.5 \mathrm{~g} \mathrm{~L}^{-1} \mathrm{BSA}$ on the half-life of the foam (D), the maximal liquid holdup of the foam $(E)$, the average bubble radius of the foam (F).

Based on the Foamscan analysis and additional reactor trials, a superficial gas velocity of 1.9 $\mathrm{mm} \mathrm{s}^{-1}$ in combination with a protein concentration of $1.75 \mathrm{~g} \mathrm{~L}^{-1}$ was chosen. These settings were a compromise between sufficient foam stability in the foam-bed photobioreactor while still allowing for reasonable foam break-up. With these settings, the foam half-life is expected 
to be between 0.5 and 1.5 hours and the foam liquid holdup approximately 5.1 to $6.4 \%$ (Figure $2.3 \mathrm{~A}$ and $\mathrm{B}$ ) when neglecting the effect of gas flow rates (as those did not had a significant effect above $1.8 \mathrm{~mm} \mathrm{~s}^{-1}$ ). The bubble size is expected to be between 0.27 and $0.42 \mathrm{~mm}$ (Figure $2.3 \mathrm{C})$.

The empty bed gas residence time is a good measure for the relative gas flow supplied to a foam-bed reactor and it allows for the comparison of different studies and reactors. The empty bed gas residence time is obtained by dividing the volume of the reactor by the volumetric gas flow rate entering the reactor. The empty bed gas residence time was 3.58 minutes, which is significantly longer than reported before for other foam-bed reactors (i.e. less than one minute) $[16,18-20,32]$. Our long residence time reflects reduced gassing, which may contribute to reduced operation costs when considering scale-up.

\subsubsection{Designing foam break-up}

In order to ensure efficient foam break-up in the foam-bed photobioreactor, various methods were experimentally tested for their suitability. These methods included bubble break-up due to natural destabilisation, foam centrifugation, mechanical disruption by a stirrer, and foam collapse due to physical contact with hydrophobic solid materials. First a brief overview of the approaches that were found not suitable for the foam-bed photobioreactor are listed.

Natural foam destabilisation is a combined result of coalescence, coarsening and drainage. This method resulted in a dry, inhomogeneous foam, instead of the desired foam destabilisation with foam collapsing from the topmost layer only. A visible protein shell remained at the maximum foam height in the reactor, originating from the proteins that were released form the bubbles' rigid stabilising films when the bubbles burst [45-47]. This effect led to protein depletion from the bulk and, consequently, the foam became less stable in time.

When using a continuous centrifuge, the foam was broken down efficiently. However, at the lowest required rotational speed for foam break-up, the microalgae also settled and accumulated in the centrifuge. A mechanical stirrer created a foam with smaller bubbles instead of collapsing the foam.

A hydrophobic sieve plate made of PDMS, described in a previous study [34], was also tested in two different configurations. When the foam entered from the bottom, the foam continuously rose in the foam breaker until reaching the plate. Some bubbles were not broken and consequently passed through the sieve, leading to liquid accumulation on the top of the sieve 
because liquid drainage was obstructed by the up-flowing gas. The latter problem was eliminated by passing the foam through the foam breaker from the top to the bottom. In this configuration the gas flow direction, and the natural drainage direction (due to gravitational force) corresponded, thus the liquid could easily pass through the sieve, although still some unbroken bubbles were left behind.

In order to further increase the foam break-up efficiency, the sieve was replaced by a packed bed column containing hydrophobic beads. The defoaming properties of solid hydrophobic particles are well studied and it appears that particles with high contact angles are particularly efficient in destabilising foams [48]. Two different bead materials were used together within the packed bed column: PDMS and PTFE beads. Furthermore, a hydrophobic coating was applied on the glass column (Sigmacote, Sigma). The contact angles of these materials in air with water were reported to be $100^{\circ}$ [49], $109^{\circ}$ [50] for PDMS, $116^{\circ}$ for PTFE [51], and $91^{\circ}$ for glass coated with Sigmacote [52]. Other potential advantages of this packed bed column design are: 1) that the foam breaking efficiency of the packed column may be increased compared to that of the sieve due to the increased contact time and area between the material and the foam; 2) that the beads applied are large enough to not mix into the liquid phase; and 3) that relatively large beads, i.e. a large pore size, can be used thereby reducing the pressure drop in the reactor. According to our knowledge, this is the first study where a solid defoaming material was used for continuous defoaming in a foam-bed reactor system.

The beads were more efficient in foam breaking compared to the sieve plate according to experiments with the foam-bed photobioreactor. The efficiency of the foam breaker was dependent on the gas flow rates and surfactant concentrations. At low surfactant concentration and reduced gas flow rates, thus low foam loads, the foam breaker worked efficiently. However, when the foam load was elevated, a foamy fluid instead of pure liquid was pumped back to the reactor. This did not seem to cause problems and a stable foam-bed could be maintained. A slight overpressure developed in the reactor due to the foam breaker, as it reduced the cross sectional area and created a resistance to the flow of the foam (approximately $4 \%$ of the cross sectional area of the reactor remained open for foam flow in between the beads inside the foam breaker). The overpressure in the reactor was always under 60 mbar. 


\subsubsection{Microalgal growth in foam-bed photobioreactor}

Chlorella sorokiniana cells were cultivated for 8 hours in the foam-bed photobioreactor. The average growth rate in the foam-bed photobioreactor during these 8 hours was $0.10 \mathrm{~h}^{-1}$. This finding was based on the increase in cell volume concentration (Figure 2.4), which corresponded with the measurements of optical density and cell number. These results indicate that microalgae can grow in liquid foams and that the photobioreactor developed is suitable for microalgae cultivation.

The growth rate achieved in the foam-bed reactor is good and comparable to other studies but lower than the maximal specific growth rate of Chlorella sorokiniana reported, which is $0.27 \mathrm{~h}$ ${ }^{-1}[53,54]$. The difference between the maximal growth rate and the growth rate reached in the foam-bed photobioreactor is related to the fact that not the whole microalgal culture is illuminated and that the average light intensity in the culture is below the saturation point, predominantly because of microalgal self-shading. More specifically, in the bulk liquid, foam breaker, and associated tubing, the cells were not receiving light to support their growth. Assuming an average of $5 \%$ liquid holdup in the foam, approximately $30 \%$ of the culture volume was not illuminated. In addition, the light intensity used was lower than in the studies where the maximum growth rate was reached [53, 54]. Moreover, biomass density was considerable resulting in microalgal self-shading.

In order to relate the achieved biomass densities to other studies, the growth was expressed as increase in dry weight concentration, for which a conversion factor of 0.5 was used to convert $\mathrm{mL}$ cell volume (Figure 2.4) to gram dry weight [55]. The biomass density on average increased from 2.1 to $4.7 \mathrm{~g} \mathrm{~L}^{-1}$ after 8 hours of growth. As a comparison, in experiments of microalgal suspension cultures the biomass density generally is 1 to $3 \mathrm{~g} \mathrm{~L}^{-1}$ at an equivalent specific growth rate $\left(0.1 \mathrm{~h}^{-1}\right)$ and in reactors of comparable thickness [36, 56-58]. In these studies higher light intensities were applied than in the present study, indicating that the foam-bed photobioreactor allows for elevated biomass density cultures.

The finding that the growth rate of Chlorella sorokiniana in a foam-bed is in the same range as in comparable suspension cultivations demonstrates that $C$. sorokiniana cultures are able to withstand the shear stresses associated to foam bubble formation and collapse. This finding is supported by the stable and high maximum quantum yield of PSII photochemistry of the microalgal culture throughout the entire experiment. This is because it is known from other studies that shear stress during bubble formation at the sparger and bubble collapse in the 
headspace might damage microalgal cells [59-61], while it was also reported that the quantum yield of PSII photochemistry decreases during excessive shear stress acting on the cells [62]. At the start of the growth experiment, microalgae were acclimated to optimal growth conditions, as shown by a high quantum yield of $0.72-0.78$. This was the quantum yield observed in a conventional flat panel photobioreactor, which served as inoculum for the foambed photobioreactor. During the 8 hours growth experiments the quantum yield did not change (Figure 2.4B), indicating that the integrity of the photosynthetic machinery of C. sorokiniana was conceivably not affected by foam bubble formation and break-up.

Quantification of growth in a foam-bed photobioreactor can easily be compromised. Hence, the following measures and measurements were done to exclude erroneous conclusions. First, the total cell volume concentration was determined by only taking into account cell diameters between 2 and $6 \mu \mathrm{m}$, thus the reported values reflect only the microalgal biomass concentration and exclude any bacterial biomass. Second, the average ratio between microalgae concentration in the foam to their concentration in the bulk liquid was found to be $0.9 \pm 0.2$, indicating that the microalgae had equal distribution over the two phases. Thus, the measurements on the bulk liquid are representative for the whole reactor including the foam section. Finally, the humidity and temperature of the inlet and outlet gas of the reactor were continuously monitored in order to quantify liquid loss due to evaporation. Approximately $1.7 \mathrm{~g}$ of water was entering the reactor as vapour via the inlet gas in 8 hours, while 1.6 to $2.1 \mathrm{~g}$ water left as vapour via the outlet gas. The results indicate that less than half a gram of water had been evaporated during the whole reactor run, which is negligible compared to the total amount of water present $(150 \mathrm{~mL})$. Thus, the possibility of the microalgae biomass concentration increasing due to evaporative loss of water can be excluded, confirming that the concentration increase observed was solely due to microalgal growth. 

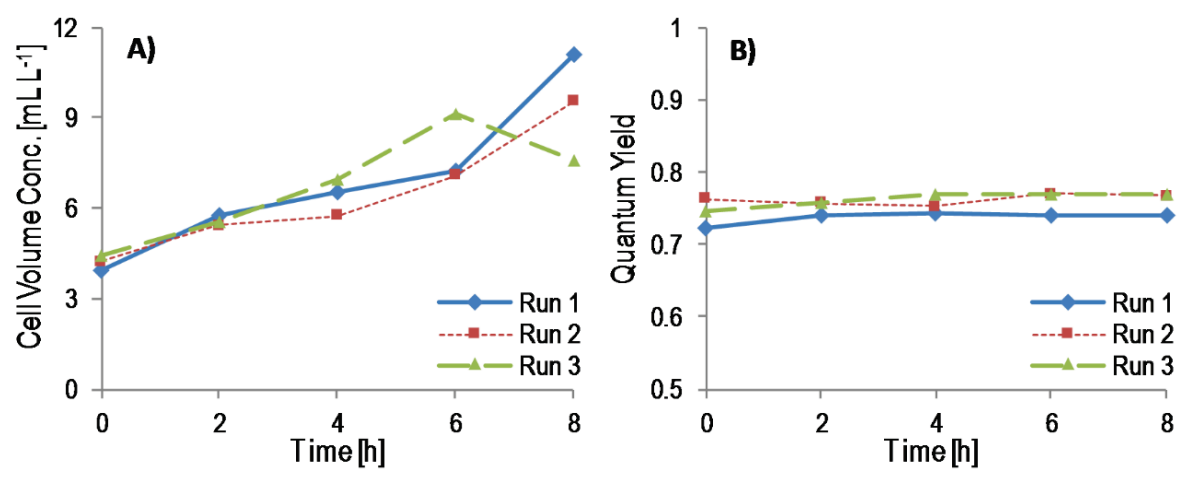

Figure 2.4. A) Microalgal growth in the foam-bed photobioreactor measured as microalgal cell volume concentration in time; B) Maximum quantum yield of PSIl photochemistry $\left(\mathrm{F}_{\mathrm{v}} / \mathrm{F}_{\mathrm{m}}\right)$ during the three growth experiments.

\subsubsection{Long-term stability of BSA -stabilized foam}

The protein concentration in the cell free supernatant of reactor samples continuously decreased during the reactor runs (Figure 2.5). This was also confirmed by visual observations of decreasing foam stability. Larger gas bubbles appeared in time and the liquid content of the foam declined. This was also confirmed by a decreased recirculation flow, implying a decrease in the amount of foam leaving the reactor and/or a decreased liquid holdup of the foam.

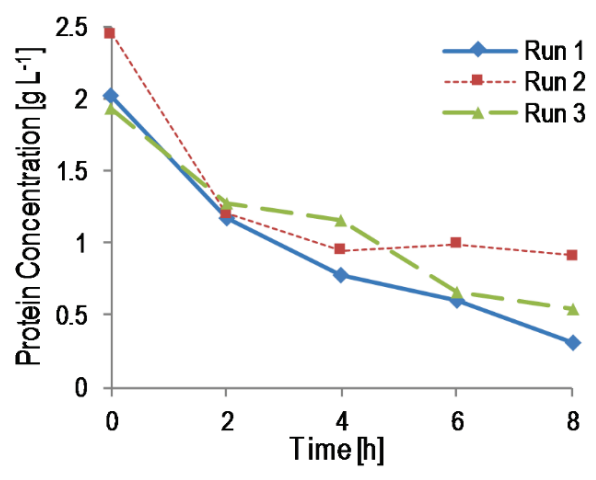

Figure 2.5. Protein concentration in the cell-free supernatant of the bulk liquid during three different 8 hour growth experiments in the foam-bed photobioreactor.

BSA molecules have a clear preference for the foam phase, indicated by the protein analysis of both the foam phase and the bulk liquid phase. While the bulk liquid phase protein concentration is decreasing below $1 \mathrm{~g} \mathrm{~L}^{-1}$ after 8 hours, the protein concentration in the foam only shows a 
small decline in time in comparison to its initial value of $\sim 2.1 \mathrm{~g} \mathrm{~L}^{-1}$ (data not shown). The observation of protein enrichment in the foam phase is in agreement with other studies [63].

The decreasing protein concentration in the bulk liquid could be due to protein aggregation because of foaming induced damage in the BSA molecules. Protein molecules experience conformational changes when foamed and, consequently, their properties and structure are altered as well, causing aggregation [42]. The aggregated protein might have been removed with the centrifugation step prior to the protein assay. Besides, several other reasons might stand behind the decline in protein concentration, including biodegradation by microorganisms present in the cultures [64], thermal degradation [65], or adsorption [66].

To test the alternative hypotheses on the origin of the decreasing protein concentration in the bioreactor experiments, additional experiments were done. Shake flasks containing BSA were inoculated with $C$. sorokiniana cultures and were placed in a dark incubator at $37^{\circ} \mathrm{C}$. The protein and microalgal cell volume concentration were continuously monitored. The results of these shake flask experiments showed that neither the algae concentration neither the BSA concentration changed during the 9 days of experiments. This revealed that BSA did not adsorb to microalgal cells in a significant extent, as the cells were removed prior to the protein measurement. Furthermore, the possibility of consumption of the protein by C. sorokiniana or by the bacterial consortium present in the cultures can be excluded. These experiments also exclude the possibility of thermal degradation of BSA as the protein concentration remained unchanged while incubated at $37^{\circ} \mathrm{C}$. The possibility of BSA adsorption in the foam breaker was also tested. A protein solution of $2 \mathrm{~g} \mathrm{~L}^{-1} \mathrm{BSA}$ was flushed through the foam breaker device including the hydrophobic PTFE and PDMS beads. The results showed that the protein concertation remains unchanged, indicating that no adsorption took place to the foam breaker device and its content.

To summarize, we think it is most likely that the decrease of foam stability in time is due to protein denaturation due to foaming. Next to this, possibly the biodegradation of the protein by bacteria also contributed to the protein decrease in the reactor, since a few bacterial cells were observed in the reactor samples by microscopic analysis. The decrease in foam stability implies that BSA is not suitable for applications where continuous re-foaming of the surfactants is required. Therefore, BSA does not meet the requirements for long-term application within foam-bed photobioreactors, revealing the need for novel surfactants. Nevertheless, BSA is a 
good foaming agent in order to study foam-based microalgal growth on a time scale of multiple hours.

\subsection{Conclusions}

The results of this study suggest that the novel foam-bed photobioreactor can be a good alternative to conventional microalgae cultivation system. A foam-bed photobioreactor was successfully developed for microalgae cultivation and its ability to support microalgal growth has been confirmed. Aiming at optimal reactor performance, foam formation and foam breakup systems are essential. BSA protein was successfully used as foam stabilising. For efficient separation of the gas and liquid phase in the foam leaving the reactor a packed bed column filled with hydrophobic beads was developed. Chlorella sorokiniana showed an average specific growth rate of $0.10 \mathrm{~h}^{-1}$ in the foam-bed photobioreactor in combination with high PSII efficiency. The biggest limitation of the foam-bed photobioreactor was the short operational time of 8 hours due to protein depletion from the bulk liquid. For long-term operation of a foambed photobioreactor, a more stable foaming agent is required. 


\section{References}

[1] R.J. Shields, I. Lupatsch, Algae for aquaculture and animal feeds, J. Anim. Sci. 21 (2012) 23-37.

[2] C. Posten, C. Walter, Microalgal biotechnology: Integration and economy, De Gruyter, Berlin, Boston, 2012.

[3] R.B. Draaisma, R.H. Wijfels, P.M. Slegers, L.B. Brentner, A. Roy, M.J. Barbosa, Food commodities from microalgae, Curr. Opin. Biotechnol. 24 (2013) 169-177.

[4] R.H. Wijfels, M.J. Barbosa, M.H. Eppink, Microalgae for the production of bulk chemicals and biofuels, Biofuels, Bioprod. Biorefin. 4 (2010) 287-295.

[5] J. Milledge, Commercial application of microalgae other than as biofuels: a brief review, Rev. Environ. Sci. Biotechnol. 10 (2011) 31-41.

[6] R.N. Singh, S. Sharma, Development of suitable photobioreactor for algae production - A review, Renew. Sust. Energ. Rev. 16 (2012) 2347-2353.

[7] F.G. Acién, J.M. Fernández, J.J. Magán, E. Molina, Production cost of a real microalgae production plant and strategies to reduce it, Biotechnol. Adv. 30 (2012) 1344-1353.

[8] C.U. Ugwu, H. Aoyagi, H. Uchiyama, Photobioreactors for mass cultivation of algae, Bioresour. Technol. 99 (2008) 4021-4028.

[9] N. Uduman, Y. Qi, M.K. Danquah, G.M. Forde, A. Hoadley, Dewatering of microalgal cultures: a major bottleneck to algae-based fuels, J. Renewable Sustainable Energy 2 (2010) 012701.

[10] C. Posten, Design principles of photo-bioreactors for cultivation of microalgae, Eng. Life Sci. 9 (2009) 165177.

[11] M. Janssen, P.P. Lamers, M. de Haan, R.H. Wijfels, Growing microalgae or cyanobacteria in liquid-based foam, International patent application No. PCT/EP2013/073065, publication number WO 2014072294 A1, filed Nov. 5 2013, issued May 15, 2014, Wageningen.

[12] A.A. Gaikwad, A.N. Bhaskarwar, Absorption of pure carbon-dioxide gas in a foam-bed reactor, Proceedings of European Congress of Chemical Engineering (ECCE-6), Copenhagen, 2007.

[13] S.R. Asolekar, P.K. Deshpande, R. Kumar, A model for a foam-bed slurry reactor, AIChE J. 34 (1988) 150154.

[14] A.N. Bhaskarwar, D. Desai, R. Kumar, General model of a foam bed reactor, Chem. Eng. Sci. 45 (1990) 1151-1159.

[15] M.B. Ripley, A.B. Harrison, W.B. Betts, R.K. Dart, A.J. Wilson, Enhanced degradation of a model oil compound in soil using a liquid foam-microbe formulation, Environ. Sci. Technol. 34 (2000) 489-496.

[16] E. Kan, M.A. Deshusses, Development of foamed emulsion bioreactor for air pollution control, Biotechnol. Bioeng. 84 (2003) 240-244.

[17] E. Kan, M.A. Deshusses, Continuous operation of foamed emulsion bioreactors treating toluene vapors, Biotechnol. Bioeng. 92 (2005) 364-371.

[18] J. Song, Y. Kim, Y. Son, J. Khim, A bioactive foam reactor for the removal of volatile organic compounds: system performance and model development, Bioprocess. Biosyst. Eng. 30 (2007) 439-446.

[19] F.G. Shahna, F. Golbabaei, J. Hamedi, H. Mahjub, H.R. Darabi, S.J. Shahtaheri, Treatment of Benzene, Toluene and Xylene Contaminated Air in a Bioactive Foam Emulsion Reactor, Chin. J. Chem. Eng. 18 (2010) 113-121.

[20] F.G. Shahna, F. Golbabaei, J. Hamedi, H. Mahjub, H.R. Darabi, S.J. Shahtaheri, A bioactive foamed emulsion reactor for the treatment of benzene-contaminated air stream, Bioprocess. Biosyst. Eng. 33 (2010) 219226.

[21] N.S. Deshpande, M. Barigou, Performance characteristics of novel mechanical foam breakers in a stirred tank reactor, J. Chem. Technol. Biotechnol. Biotechnology 74 (1999) 979-987.

[22] Y. Chisti, Animal-cell damage in sparged bioreactors, Trends Biotechnol. 18 (2000) 420-432.

[23] A.C. Martinez, E. Rio, G. Delon, A. Saint-Jalmes, D. Langevin, B.P. Binks, On the origin of the remarkable stability of aqueous foams stabilised by nanoparticles: link with microscopic surface properties, Soft Matter 4 (2008) 1531-1535.

[24] K. Miyamoto, O. Wable, J. Benemann, Vertical tubular reactor for microalgae cultivation, Biotechnol. Lett. 10 (1988) 703-708.

[25] A.A. Gaikwad, N. Challapalli, A.N. Bhaskarwar, Carbonation of Barium Sulfide in a Foam-Bed Reactor, Chem. Eng. Commun. 197 (2010) 804-829.

[26] A. Varshney, P. Agrawal, A.N. Bhaskarwar, Gas absorption with zero-order chemical reaction in a foam-bed reactor, Chem. Eng. Sci. 58 (2003) 3413-3424. 
[27] G.S. Reddy, A.N. Bhaskarwar, Modeling of Gas Absorption with a Zero-order Chemical Reaction in a Foambed Reactor: Comparison with Oxidation of Sodium Dithionite, Chem. Eng. Sci. 58 (2003) 3413-3424

[28] M. Barigou, Foam rupture by mechanical and vibrational methods, Chem. Eng. Technol. 24 (2001) 659-663.

[29] S. Takesono, M. Onodera, A. Ito, M. Yoshida, K. Yamagiwa, A. Ohkawa, Mechanical control of foaming in stirred-tank reactors, J. Chem. Technol. Biotechnol. 76 (2001) 355-362.

[30] William H. Echols, Apparatus for breaking up a foam, U.S, Patent 3,298,615, filed Sept. 25 1964, issued Jan. 17, 1967, Fort Foote Village,

[31] S. Takesono, M. Onodera, M. Yoshida, K. Yamagiwa, A. Ohkawa, Performance characteristics of mechanical foam-breakers fitted to a stirred-tank reactor, J. Chem. Technol. Biotechnol. 78 (2003) 48-55.

[32] E. Kan, M.A. Deshusses, Cometabolic degradation of TCE vapors in a foamed emulsion bioreactor, Environ. Sci. Technol. 40 (2006) 1022-1028.

[33] D.O. Hitzman, E.H. Wegner, Methanol foam fermentation to single cell protein by Pseudomonas methanica, U.S. Patent RE30,543, filed Jan 21, 1977, issued March 10, 1981, Bartlesville, Okla.

[34] M. Ishida, R. Haga, N. Nishimura, H. Matuzaki, R. Nakano, High cell density suspension culture of mammalian anchorage independent cells: Oxygen transfer by gas sparging and defoaming with a hydrophobic net, Cytotechnology 4 (1990) 215-225.

[35] Y.-P. Wang, K. Yuan, Q.-L. Li, L.-P. Wang, S.-J. Gu, X.-W. Pei, Preparation and characterization of poly (Nisopropylacrylamide) films on a modified glass surface via surface initiated redox polymerization, Mater. Lett. 59 (2005) 1736-1740.

[36] A.M. Kliphuis, L. de Winter, C. Vejrazka, D.E. Martens, M. Janssen, R.H. Wijfels, Photosynthetic efficiency of Chlorella sorokiniana in a turbulently mixed short light-path photobioreactor, Biotechnol. Prog. 26 (2010) 687-696. [37] T. de Mooij, M. Janssen, O. Cerezo-Chinarro, J.H. Mussgnug, O. Kruse, M. Ballottari, R. Bassi, S. Bujaldon, F.-A. Wollman, R.H. Wijfels, Antenna size reduction as a strategy to increase biomass productivity: a great potential not yet realized, J. Appl. Phycol. 27 (2014) 1063-1077.

[38] N.R. Baker, Chlorophyll fluorescence: a probe of photosynthesis in vivo, Annu. Rev. Plant Biol. 59 (2008) 89113.

[39] F.J. Lech, M.B. Meinders, P.A. Wierenga, H. Gruppen, Comparing foam and interfacial properties of similarly charged protein-surfactant mixtures, Colloids Surf., A 473 (2015) 18-23.

[40] P. Asharani, Y.L. Wu, Z. Gong, S. Valiyaveettil, Toxicity of silver nanoparticles in zebrafish models, Nanotechnology 19 (2008) 255102.

[41] P. Wilde, A. Mackie, F. Husband, P. Gunning, V. Morris, Proteins and emulsifiers at liquid interfaces, Adv. Colloid Interface Sci. 108 (2004) 63-71.

[42] J. Clarkson, Z. Cui, R. Darton, Protein denaturation in foam: II. Surface activity and conformational change, J. Colloid Interface Sci. 215 (1999) 333-338.

[43] P. Stevenson, Hydrodynamic theory of rising foam, Miner. Eng. 20 (2007) 282-289.

[44] S. Boonyasuwat, S. Chavadej, P. Malakul, J.F. Scamehorn, Surfactant recovery from water using a multistage foam fractionator: Part I effects of air flow rate, foam height, feed flow rate and number of stages, Sep. Sci. Technol. 40 (2005) 1835-1853.

[45] J.F. Zayas, Foaming properties of proteins, in: J.F. Zayas, Functionality of proteins in food, Springer, Berlin, Heidelberg, 1997, pp. 260-309.

[46] A. Saint-Jalmes, M.L. Peugeot, H. Ferraz, D. Langevin, Differences between protein and surfactant foams: Microscopic properties, stability and coarsening, Colloids Surf., A 263 (2005) 219-225.

[47] M.A. Bos, T. van Vliet, Interfacial rheological properties of adsorbed protein layers and surfactants: a review, Adv. Colloid Interface Sci. 91 (2001) 437-471.

[48] R. Pugh, Foaming, foam films, antifoaming and defoaming, Adv. Colloid Interface Sci. 64 (1996) 67-142.

[49] J. Bongaerts, K. Fourtouni, J. Stokes, Soft-tribology: Iubrication in a compliant PDMS-PDMS contact, Tribol. Int. 40 (2007) 1531-1542.

[50] S. Bhattacharya, A. Datta, J.M. Berg, S. Gangopadhyay, Studies on surface wettability of poly (dimethyl) siloxane (PDMS) and glass under oxygen-plasma treatment and correlation with bond strength, J.

Microelectromech. Syst. 14 (2005) 590-597.

[51] T. Zawodzinski, S. Gottesfeld, S. Shoichet, T. McCarthy, The contact angle between water and the surface of perfluorosulphonic acid membranes, J. Appl. Electrochem. 23 (1993) 86-88.

[52] S.N. Krylov, N.J. Dovichi, Single-cell analysis using capillary electrophoresis: influence of surface support properties on cell injection into the capillary, Electrophoresis 21 (2000) 767-773. 
[53] M. Janssen, T.C. Kuijpers, B. Veldhoen, M.B. Ternbach, J. Tramper, L.R. Mur, R.H. Wijfels, Specific growth rate of Chlamydomonas reinhardtii and Chlorella sorokiniana under medium duration light/dark cycles: 13-87 s, J. Biotechnol. 70 (1999) 323-333.

[54] J. Van Wagenen, S.L. Holdt, D. De Francisci, B. Valverde-Pérez, B.G. Plósz, I. Angelidaki, Microplate-based method for high-throughput screening of microalgae growth potential, Bioresour. Technol. 169 (2014) 566-572.

[55] M. Janssen, R. Wijffels, U. von Stockar, Biocalorimetric monitoring of photoautotrophic batch cultures, Thermochim. Acta 458 (2007) 54-64.

[56] M. Cuaresma, M. Janssen, C. Vílchez, R.H. Wijffels, Horizontal or vertical photobioreactors? How to improve microalgae photosynthetic efficiency, Bioresour. Technol. 102 (2011) 5129-5137.

[57] M.C. Franco, M.F. Buffing, M. Janssen, C.V. Lobato, R.H. Wijffels, Performance of Chlorella sorokiniana under simulated extreme winter conditions, J. Appl. Phycol. 24 (2012) 693-699.

[58] M. Cuaresma, M. Janssen, C. Villchez, R.H. Wijffels, Productivity of Chlorella sorokiniana in a short light-path (SLP) panel photobioreactor under high irradiance, Biotechnol. Bioeng. 104 (2009) 352-359.

[59] J.M.S. Rocha, J.E.C. Garcia, M.H.F. Henriques, Growth aspects of the marine microalga Nannochloropsis gaditana, Biomol. Eng. 20 (2003) 237-242.

[60] W. Hu, R. Gladue, J. Hansen, C. Wojnar, J.J. Chalmers, The Sensitivity of the Dinoflagellate

Crypthecodinium cohnii to Transient Hydrodynamic Forces and Cell-Bubble Interactions, Biotechnol. Progr. 23 (2007) 1355-1362.

[61] T.M. Sobczuk, F.G. Camacho, E.M. Grima, Y. Chisti, Effects of agitation on the microalgae Phaeodactylum tricornutum and Porphyridium cruentum, Bioprocess. Biosyst. Eng. 28 (2006) 243-250.

[62] M. Leupold, S. Hindersin, G. Gust, M. Kerner, D. Hanelt, Influence of mixing and shear stress on Chlorella vulgaris, Scenedesmus obliquus, and Chlamydomonas reinhardtii, J. Appl. Phycol. 25 (2013) 485-495.

[63] R.W. Schnepf, E.L. Gaden, Foam fractionation of proteins: concentration of aqueous solutions of bovine serum albumin, J. Biochem. Microbiol. Tech. Eng. 1 (1959) 1-11.

[64] K. Watanabe, N. Takihana, H. Aoyagi, S. Hanada, Y. Watanabe, N. Ohmura, H. Saiki, H. Tanaka, Symbiotic association in Chlorella culture, FEMS Microbiol. Ecol. 51 (2005) 187-196.

[65] I. Hayakawa, J. Kajihara, K. Morikawa, M. Oda, Y. Fujio, Denaturation of bovine serum albumin (BSA) and ovalbumin by high pressure, heat and chemicals, J. Food Sci. 57 (1992) 288-292.

[66] H.B. Bull, Adsorption of bovine serum albumin on glass, BBA 19 (1956) 464-471. 


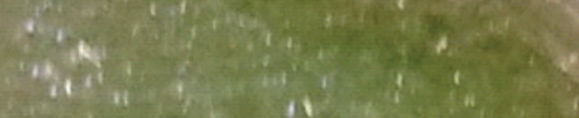




\title{
Chapter 3
}

\section{Surfactant selection for a liquid foam-bed}

\author{
photobioreactor
}

This chapter is published as:

A. Janoska and M. Vázquez, M. Janssen, R.H. Wijffels, M. Cuaresma, C. Vílchez, Surfactant selection for a liquid foam-bed photobioreactor, Biotechnol. Prog., doi: 10.1002/btpr.2614 


\begin{abstract}
A novel liquid foam-bed photobioreactor has been shown to hold potential as an innovative technology for microalgae production. In this study a foam stabilizing agent has been selected which fits the requirements of use in a liquid foam-bed photobioreactor. Four criteria were used for an optimal surfactant: the surfactant should have good foaming properties, should not be rapidly biodegradable, should drag up microalgae in the foam formed, and it should not be toxic for microalgae. Ten different surfactants (non-ionic, cationic and anionic) and two microalgae genera (Chlorella and Scenedesmus) were compared on the above-mentioned criteria. The comparison showed the following facts. Firstly, poloxameric surfactants (Pluronic F68 and Pluronic P84) have acceptable foaming properties described by intermediate foam stability and liquid holdup and small bubble size. Secondly, the natural surfactants (BSA and Saponin) and Tween 20 were easily biodegraded by bacteria within 3 days. Thirdly, for all surfactants tested the microalgae concentration is reduced in the foam phase compared to the liquid phase with exception of the cationic surfactant CTAB. Lastly, only BSA, Saponin, Tween 20 and the two Pluronics were not toxic at concentrations of $10 \mathrm{CMC}$ or higher. The findings of this study indicate that the Pluronics (F68 and P84) are the best surfactants regarding the above-mentioned criteria. Since Pluronic F68 performed slightly better, this surfactant is recommended for application in a liquid foam-bed photobioreactor.
\end{abstract}




\subsection{Introduction}

In order to benefit from the great potential of microalgae for the production of specialties for food and aquaculture, biofuels and bulk chemicals, further progress is needed in the field of microalgae cultivation [1-5]. With the intention to develop a low-cost microalgae production system, a liquid foam-bed photobioreactor was developed and tested [6]. In order to create foam in the foam-bed photobioreactor, the addition of surfactants is required. Due to the continuous gas supply through a surfactant-containing media with microalgae, the generated foam is rising in the foam-bed photobioreactor, dragging the microalgal cells upwards. The foam-bed photobioreactor is illuminated and the gas phase is enriched with carbon dioxide $\left(\mathrm{CO}_{2}\right)$. As such, the microalgal cells can rapidly grow in the liquid layers within the foam. To prevent $\mathrm{CO}_{2}$ limitation for the growing microalgal cultures, the foam has to be broken after a certain time period and regenerated with fresh $\mathrm{CO}_{2}$-rich gas.

Previous research on the liquid foam-bed photobioreactor showed that it was possible to grow microalgae at a specific growth rate of $0.1 \mathrm{~h}^{-1}$ [6]. These growth experiments only lasted for 8 hours due to a decrease in foam stability in time. Since a protein, Bovine Serum Albumin, was used as a foam stabilising agent, its foamability was declining due to biodegradation and/or possible denaturation and consequent damage caused by the foaming process [6]. Long term operation of the foam-bed photobioreactor is crucial for a successful implementation of this innovative reactor system and to eventually compete with other photobioreactors. Therefore, more stable foam stabilising agents have to be selected which also match all other requirements for application in liquid foam-bed photobioreactors.

Foam stabilising agents are a highly diverse group, which is comprised of low and high molecular weight surfactants. Four different types are distinguished according to their electrostatic nature: anionic, cationic, non-ionic, and zwitterionic surfactants. In addition to this classification, synthetic and natural surfactants (e.g. proteins, saponins) are distinguished. Surfactant molecules differ in their structure, even if they belong to the same group, resulting in highly varying properties, such as foaming properties, biodegradability, and also altered interactions with algal cells.

In this study 4 specific characteristics of surfactants have been identified based on which the optimal surfactant can be selected. First characteristic is related to their foaming properties, including foam stability, liquid content, bubble size and surfactant stability. An ideal surfactant 
for the liquid foam-bed photobioreactor should create foams with intermediate liquid content and stability in order to create a stable, but breakable foam. Homogenous foams with small bubble sizes are preferred in order to maximize mass transfer. These properties are dependent on the surfactant structure, e.g. the length of the hydrophobic chain was shown to influence the surface activity and motility of the surfactant, determining the foam properties [7]. Moreover, in order to ensure long-term foam forming capacity, the surfactant molecules themselves must also be stable in order to be able to repeatedly form foams during long term operations of liquid foam-bed photobioreactors.

The second characteristic for surfactant selection is the biodegradation rate of the surfactant. Many surfactants can be biologically degraded [8,9], which means that the surfactants can be used as carbon and energy source by bacteria. However, biodegradation is directly related to the molecule's structure. For example, the more branched the carbon chain of a surfactant molecule is, the slower its biodegradation rate becomes [10]. Also the aromatic nature of a surfactant molecule can influence biodegradation rates [11]. Considering different bacteria populations grow naturally associated to growing microalgae [12], the optimal surfactant should show a low biodegradability in order to minimize surfactant losses associated to its bacterial consumption.

The third characteristic is the partitioning of microalgae to the foam phase. It is evident that the foams formed by the chosen surfactant must be enriched with microalgae. Microalgal partitioning towards the foam is determined primarily by hydrostatic interaction between the air bubbles and the microalgae, thus algal cell surface hydrophobicity might play a role [13]. Besides, the electrostatic charging of the algal cells and the surfactant molecules are crucial. Surfactants can also render algal cell surface hydrophobicity by attaching to cells via electrostatic interactions [14], or alternatively the bubbles can own an electrostatic charge due to the charged surfactant molecules used for foam stabilisation [15]. Cationic surfactants, for example, have a higher algal foam partitioning as they attach the strongest to the usually negatively charged algal cell [14].

The fourth characteristic of a surfactant which is essential for its application in a liquid foambed photobioreactor is its toxicity to microalgae. The reported toxic effects of a surfactant on microalgal growth are a reduction in cell density, growth rate, cell motility, chlorophyll content, and the inhibition of the photosynthetic activity [16-19]. The extent of this toxicity is dependent 
on surfactant type, concentration, and the microalgal species involved [20]. For example, cationic surfactants are, in general, more toxic than their anionic or non-ionic partners [21].

Although some basic principles are understood, it is still difficult to predict surfactants properties and their interactions with different algal species solely based on their structure and theory. The aim of this study is therefore to empirically select a suitable foam stabilising agent for the liquid foam-bed photobioreactor that can enable elongated cultivations of days to weeks. In the current study 10 different surfactants were evaluated in terms of measured foaming properties, biodegradability, algal partitioning towards the foam and toxicity to microalgae. For this, Chlorella sorokiniana and Scenedesmus obliquus were used according to their robustness and diverse array of potential applications [22, 23]. Based on these results the best surfactant for the application in foam-bed photobioreactors was selected.

\subsection{Materials and methods}

\subsubsection{Surfactants}

Ten different surfactants were evaluated for their suitability for the liquid foam-bed photobioreactor. The selected surfactants contain two natural surfactants and 8 synthetic surfactants: six non-ionic (Pluronics F68 and P84, Tergitols NP9 and TMN6, Triton X-100 and Tween 20), one cationic (CTAB), one anionic (SDS). The natural surfactants were Saponin and a reference protein surfactant, Bovine Serum Albumin [6]. All the surfactants used are listed in Appendix 3.A. The comparison of these surfactants was done at surfactant specific concentrations, taking into account their critical micelle concentration (CMC). The CMC is the concentration above which the surfactant molecules start forming micelles in the liquid phase. The CMC values of the different surfactants are included in Appendix 3.A. These CMC values were collected from literature and/or the product information sheets.

\subsubsection{Microalgae, cultivation media and cultivation conditions}

Chlorella sorokiniana (CCAP 211/8K) was obtained from the Culture Collection of Algae and Protozoa, Oban, Scotland and Scenedesmus obliquus from the Culture Collection of Fitoplancton Marino S.L., Cádiz, Spain.

For the algae partitioning assays, C. sorokiniana was grown on 3 times concentrated M8a media, in which the $\mathrm{N}$ and $\mathrm{P}$ content were further modified to enable high biomass densities compared to the M8a recipe described previously[24] (see Appendix 3.B). The growth medium 
for S. obliquus was based on the medium from Breuer et al. [25], but further modified in order to reach higher biomass densities. Both microalgae were grown in 250-300 mL shake flasks placed in an orbital shaker in an incubator. The incubator settings were the following for $C$. sorokiniana: $37^{\circ} \mathrm{C}, 454 \mu \mathrm{mol} \mathrm{m} \mathrm{s}^{-1}, 120 \mathrm{rpm}$ and $4 \% \mathrm{CO}_{2}$, and for S. obliquus: $25^{\circ} \mathrm{C}, 120$ $\mu \mathrm{mol} \mathrm{m} \mathrm{s}^{-2}, 80-100 \mathrm{rpm}$ and $2.5 \% \mathrm{CO}_{2}$.

The culture media employed for the toxicity assays of surfactants to microalgae were M8a media [22] with $3 \mathrm{~g} \mathrm{~L}^{-1}$ of urea for C. sorokiniana, and modified BG11 media for S. obliquus [26]. For the biodegradability assays, S. obliquus was maintained in a modified M8a medium in which urea was replaced by $\mathrm{KNO}_{3}\left(3 \mathrm{~g} \mathrm{~L}^{-1}\right)$ in order to avoid the presence of any other carbon source in the media apart from the surfactants. Unless otherwise indicated, for the biodegradability and toxicity experiments both strains were maintained in linear phase cultures in shake flasks placed in a growth chamber at $25^{\circ} \mathrm{C}$. The cultures were continuously illuminated at $80 \mu \mathrm{mol}$ photons $\mathrm{m}^{-2} \mathrm{~s}^{-1}$ with fluorescent lamps and bubbled with air enriched with $2.5 \%$ (v/v) $\mathrm{CO}_{2}$.

\subsubsection{Surfactant foaming properties}

Foaming properties of the different surfactant solutions were determined by an automatized foaming device (FoamScan, Teclis- IT Concept, Logessaigne, France), as described previously [6]. Foam stability is measured in terms of the time elapsed until half of the foam volume had collapsed, and this will be referred to as the foam half-life. The liquid holdup of the foam represents the amount of liquid incorporated in the foam at the moment when the foam reached its desired volume and the gas supply stopped. All experiments were performed at a temperature between 28 and $30{ }^{\circ} \mathrm{C}$. The gas flow rate employed was $400 \mathrm{~cm}^{3} \mathrm{~min}^{-1}$, resulting in $2.4 \mathrm{~mm} \mathrm{~s}^{-1}$ superficial gas velocity. Two different concentrations were used for each surfactant: 1 and 5 CMC. BSA was measured at $1 \mathrm{~g} \mathrm{~L}^{-1}$ and $5 \mathrm{~g} \mathrm{~L}^{-1}$ at higher temperatures of $37^{\circ} \mathrm{C}$ [6]. The experiments were performed in duplicates.

The bubble size was analysed from pictures taken 30 seconds after the gas flow had stopped, at a height of $8 \mathrm{~cm}$ above the gas distributor. The mean bubble size and the standard deviation were calculated by an image analysis software (Foamscan) for each measurement, and the standard deviations and the average of the means of the two independent measurements were calculated. 
The stability of the surfactants during repetitive foaming was assessed by comparing the upflow speed of foams formed by fresh surfactant solution and the same solution after 20 continual cycles of foam production and subsequent deconstruction. Foam was produced from $200 \mathrm{~mL}$ surfactant solution in a $1 \mathrm{~L}$ glass graduated cylinder by distributing air through two smallbubble diffusers at a superficial velocity of $3.68 \mathrm{~mm} \mathrm{~s}^{-1}$. Surfactant solutions were prepared in M8a culture medium adjusted to $\mathrm{pH}$ 6.8. Foam up-flow speeds were calculated for a 30 seconds foaming time by measuring the foam level. Each surfactant test was performed in a maximum of 10 hours to prevent losses of foaming properties due to other causes (e.g., biodegradability, light sensitivity). The minimum surfactant concentration resulting in maximal foam-up flow speed $\left(C_{\min }\right)$ was determined and 1.1 times $C_{\min }$ was used in the stability tests. $C_{\min }$ values (expressed in terms of $\mathrm{CMC}$ ) found for the different surfactants were: BSA, 5; CTAB, 0.5; Pluronic F68, 5; Pluronic P84, 4.25; Saponin, 0.5; SDS, 0.08; Tergitol NP9, 1; Tergitol TMN6, 1; Triton X-100, 0.75, and Tween 20, 0.5.

\subsubsection{Surfactant biodegradability}

The biodegradability of the surfactants was indirectly determined by following bacterial growth. Bacterial growth was studied at 1 and $10 \mathrm{CMC}$ surfactant concentrations, since in a foam-bed photobioreactor, concentrations within these limits are expected. The concentration of $1 \mathrm{CMC}$ still allows for acceptable foam formation, and possible inhibitory effects on bacterial cells are minimized. A high concentration of $10 \mathrm{CMC}$ was chosen in order to assess promoting and/or inhibiting surfactant effects on bacterial growth. The experiments were performed in duplicates in $100 \mathrm{~mL}$ shake flasks. Surfactant solutions were prepared in M8a media, with nitrate as nitrogen source, and they were sterilized by filtration. Each surfactant solution was inoculated with $10 \mathrm{~mL}$ of Scenedesmus culture supernatant, which naturally contains microalgae-associated bacteria. The cultures were placed in an orbital shaker at $37^{\circ} \mathrm{C}$ and 115 $\mathrm{rpm}$. Bacterial growth was measured as the increment of optical density at $600 \mathrm{~nm}\left(O D_{600}{ }^{\text {culture }}\right)$ after 3 days $\left(\mathrm{t}_{3}\right)$, which was normalized by the initial optical density of each solution $\left(O D_{600, t 0^{\text {culture }}}\right)$, as shown in Equation 3.1. In order to avoid errors related to turbidity changes in the surfactant solutions during the experimental time, blank cultures with the different surfactant solutions (not inoculated) were prepared $\left(O D_{600}{ }^{\text {blank }}\right)$.

OD increase factor $=\frac{\left(O D_{600, t 3}^{\text {culture }}-O D_{60,0,3}^{\text {blank }}\right)-\left(O D_{600, t 0}^{\text {culture }}-O D_{600, t 0}^{\text {blank }}\right)}{\left(O D_{60, t 0}^{\text {culture }}-O D_{600, t 0}^{\text {blak }}\right)} \quad$ Equation 3.1 


\subsubsection{Microalgae partitioning towards the foam phase}

In this study the partitioning of microalgae towards the foam $\left(P_{x}^{\text {foam }}\right)$ was expressed as the ratio of the microalgae concentration in the foam liquid phase $\left(C_{x}{ }^{\text {foam }}\right)$ over the initial microalgae concentration in the liquid phase used to form the foam $\left(C_{x}\right.$, initial $)$, as presented in Equation 3.2.

$$
P_{x}^{\text {foam }}=\frac{c_{x}^{\text {foam }}}{C_{x}^{\text {initial }}}
$$

For the definition of $P_{x}^{\text {foam }}$ the initial biomass concentration is considered instead of the concentration in the bulk liquid since the bulk liquid biomass concentration is continuously changing with increasing foam volume. This definition of $P_{x}^{\text {foam }}$ allows for comparison between different systems with different dimensions.

Microalgae-surfactant mixtures were prepared by adding the surfactant to the microalgal culture (C. sorokiniana or S. obliquus). This mixture was foamed in a $2.5 \mathrm{~L}$ glass tank $(20.5 \times 3 \times 40 \mathrm{~cm}$, width $\times$ depth $\times$ height). Silicon tubing (internal diameter of $4 \mathrm{~mm}$, wall thickness of $2 \mathrm{~mm}$ ) punctured by a $0.45 \mathrm{~mm}$ needle at $1.4 \mathrm{~cm}$ intervals served as gas distributor. This tubing was placed one $\mathrm{cm}$ above the bottom of the glass tank over the complete width. A gas flow rate of $1000 \mathrm{~mL} \mathrm{~min}^{-1}$ was supplied to the surfactant solutions containing microalgae to allow foam formation. The volume of the algae suspension was $200 \mathrm{~mL}$ for $C$. sorokiniana experiments, and $100 \mathrm{~mL}$ for $S$. obliquus. The difference in the volumes was due to the fast settling of $S$. obliquus cells underneath the gas distributor. For this reason, the volume underneath the gas distributor was filled with $100 \mathrm{~mL}$ of solidified Agarose gel (5\%), and only $100 \mathrm{~mL}$ algae suspension was used to keep the same liquid height before foaming. The surfactant concentration was $5 \mathrm{CMC}$ in order to ensure sufficient foam formation, except for Saponin where $5 \mathrm{CMC}$ did not generate enough foam for the measurements; thus, $10 \mathrm{CMC}$ was used. BSA was tested at a concentration of $1.75 \mathrm{~g} \mathrm{~L}^{-1}$, which represents the concentration applied previously in a liquid foam-bed photobioreactor [6]. For each surfactant, duplicate foaming experiments were performed.

The experiments with C. sorokiniana were started at an optical density (OD) of 15 to 20 (i.e. $C_{x}$, initial), while the experiments with $S$. obliquus were started with an OD of 2. Foam was allowed to rise in the glass tank, and the foam flowing out on top was collected. In each experiment, four different foam samples of $150 \mathrm{~mL}$ were taken, provided sufficient foam was formed. The first $150 \mathrm{~mL}$ of foam was discarded and the remaining three foam fractions were 
allowed to collapse in cups. A sample was taken from the collapsed foam and the OD was measured in duplicate. Afterwards, these OD values were averaged for the three different foam fractions, and the two duplicate experiments. The optical density was measured at $750 \mathrm{~nm}$ in a spectrophotometer (Hach Lange DR6000, Germany). As SDS solutions were opaque the cell concentration was determined by cell counting: microscopic cell count using counting chamber and automatic cell counting with Beckman Coulter Multisizer 3 employing a $50 \mu \mathrm{m}$ aperture tube. In order to evaluate the differences in partitioning between the different surfactants, t-tests were done with individual pairs.

\subsubsection{Toxicity of surfactants to microalgae}

The toxic effects of surfactants on the selected microalgal strains were tested at the following surfactant concentrations: $0.1,0.5,1,2.5,5,10,15,25$ and $50 \mathrm{CMC}$, provided the surfactant was still soluble at that concentration. Cultures were prepared in shake flasks containing 300 $\mathrm{mL}$ of surfactant solution prepared in the corresponding microalgal culture medium and inoculated with microalgae cells growing in linear phase. Microalgal cultures were prepared in triplicate under sterile conditions and biomass concentration was adapted in order to get an initial optical density of 0.8 at $750 \mathrm{~nm}$. The cultures were not aerated in order to prevent foam formation. Instead of bubbling, the cultures were magnetically stirred at a speed of $200 \mathrm{rpm}$ in order to maintain the homogeneity of the suspensions. Optical density at $750 \mathrm{~nm}$ (UV/ Visible spectrophotometer, Evolution 201, ThermoFisher, United States) and maximal photosynthetic efficiency of Photosystem II (AquaPen-C AP-C 100, Photon Systems Instruments, Germany) were measured daily for a minimum of 3 days.

\subsection{Results and Discussion}

\subsubsection{Surfactant foaming properties}

In order to determine the foaming properties of the different surfactant solutions, foam volume half-life, liquid holdup in the foam, and bubble size of the foam were analysed for ten surfactants at two different concentrations, $1 \mathrm{CMC}$ and $5 \mathrm{CMC}$. A concentration of $1 \mathrm{CMC}$ is the minimal concentration required in order to reach the maximal reduction in surface tension and, as such, good foaming characteristics [27]. A concentration of 5 CMC was also used to ensure that depletion does not occur in the liquid phase (>1 CMC). From the data in Figure 3.1A, it is apparent that at a surfactant concentration of 5 CMC, CTAB and Saponin formed the most stable foams. The foam volume half-life was more than 2 hours for these surfactants. The 
exact half-life value was not determined since the experiments were terminated after 2 hours. Besides CTAB and Saponin, long foam volume half-life was observed for BSA and Tergitol NP9 at 5 CMC concentration. In contrast, Tergitol TMN6 and Tween 20 foams showed rapid destabilisation, as exemplified by a foam half-life of less than 10 minutes at $5 \mathrm{CMC}$. The surfactant SDS also resulted in unstable foams and at $1 \mathrm{CMC}$ it did not reach the foam height required for the analysis.

Most surfactants showed increased foam stability at $5 \mathrm{CMC}$ in comparison to $1 \mathrm{CMC}$ (Figure 3.1A). Since the surface tension of a surfactant solution does not change above CMC, the foaming properties are also expected to be unchanged [28]. The decrease in stability when decreasing the concentration from 5 to $1 \mathrm{CMC}$ might be due to the depletion of surfactant molecules from the liquid phase at $1 \mathrm{CMC}$. The surfactant molecules, due to their amphiphilic nature, partition to the foam phase when gas is introduced to the solution, thus the amount of surfactant molecules is reduced in the bulk liquid remaining underneath the foam. The depletion effect is especially relevant when large foam volumes are formed, or the initial surfactant concentration is low [29]. Furthermore, the rate of diffusion of the surfactant molecules to the interface also influences the foam stability and thereby might result in further foam stability increase above CMC. When the surfactant transport rate is slow, foam will be formed with reduced amount of adsorbed surfactant molecules, thus the foam stability will also be reduced [30]. Finally, the change in foaming properties above CMC might be explained by further foam stabilisation due to the presence of micelles in the thin films [31]. Consequently, at higher surfactant concentrations (>CMC) more stable and more wet foam can be formed.

The liquid holdup measured in the foam is presented in Figure 3.1B. The highest holdups were obtained with BSA, Pluronic F68, Tergitol TMN6 and Tween 20 at 5 CMC. These foams had a liquid holdup between 7.5 and 8.5\%. Under the same conditions (5 CMC), the lowest liquid holdup was $2.9 \%$ for Saponin foams. As illustrated in Figure 3.1B, the liquid content of the foam is influenced by surfactant concentration [32], with generally lower liquid holdup at 1 $\mathrm{CMC}$ in comparison to $5 \mathrm{CMC}$. As explained before this effect is probably related to surfactant depletion, surfactant diffusion limitation or micelle-based stabilisation. Next to foam stability and liquid holdup, the bubbles sizes were analysed (Figure 3.1C). In general, an increased surfactant concentration leads to a small reduction in bubble size, although for some surfactants the bubble size did not change with surfactant concentrations above CMC. Tergitol TMN6 formed foams with the smallest bubble sizes, below $0.2 \mathrm{~mm}$ radius. The largest bubbles were formed by Saponin at $5 \mathrm{CMC}$, with almost $0.5 \mathrm{~mm}$ average bubble radius. The standard 
deviations in Figure 3.1C can be regarded as a measure of the uniformity of the foam: bigger error bars mean a more heterogeneous foam, while at homogeneous foams the error bars are minimal. Generally, foams with high bubble size uniformity are more stable due to the reduced Laplace pressure between the adjacent bubbles [33]. When comparing the different surfactants, generally smaller bubble sizes were related to increased liquid holdup values and this correlation was even more dominant when considering only one surfactant at the two different concentrations (Figure 3.1B and 3.1C). Therefore, we can conclude that increasing surfactant concentration results in the combined effects of decreased bubble size and increased liquid holdup and foam stability, corresponding to previous observations [34].

Besides foam stability, liquid content and bubble size, surfactant stability against foaming is an important aspect. In previous research it was shown that BSA is not able to produce foams for elongated time periods [6]. Now we show that this phenomenon is not necessarily due to bacterial biodegradation of the molecule, but is more likely related to the instability of the surfactant during continuous foaming. Figure 3.1D shows the initial and final foam up-flow speed of each surfactant after 20 cycles of foam production and deconstruction. A reduction in this parameter could denote a loss of surfactant foamability, and therefore, of surfactant stability. The evolution of the foam up-flow speed during the 20 cycles (data not shown) revealed a dramatic drop of the up-flow speed of BSA within a short time range, with a reduction of $56 \%$ of the original value after the first three cycles. The mechanism behind the reduction of BSA foam stability is suggested to be a consequence of conformational changes in the molecule during foaming [35]. For all other surfactants no significant decrease in foam stability was observed after 20 foaming cycles. 

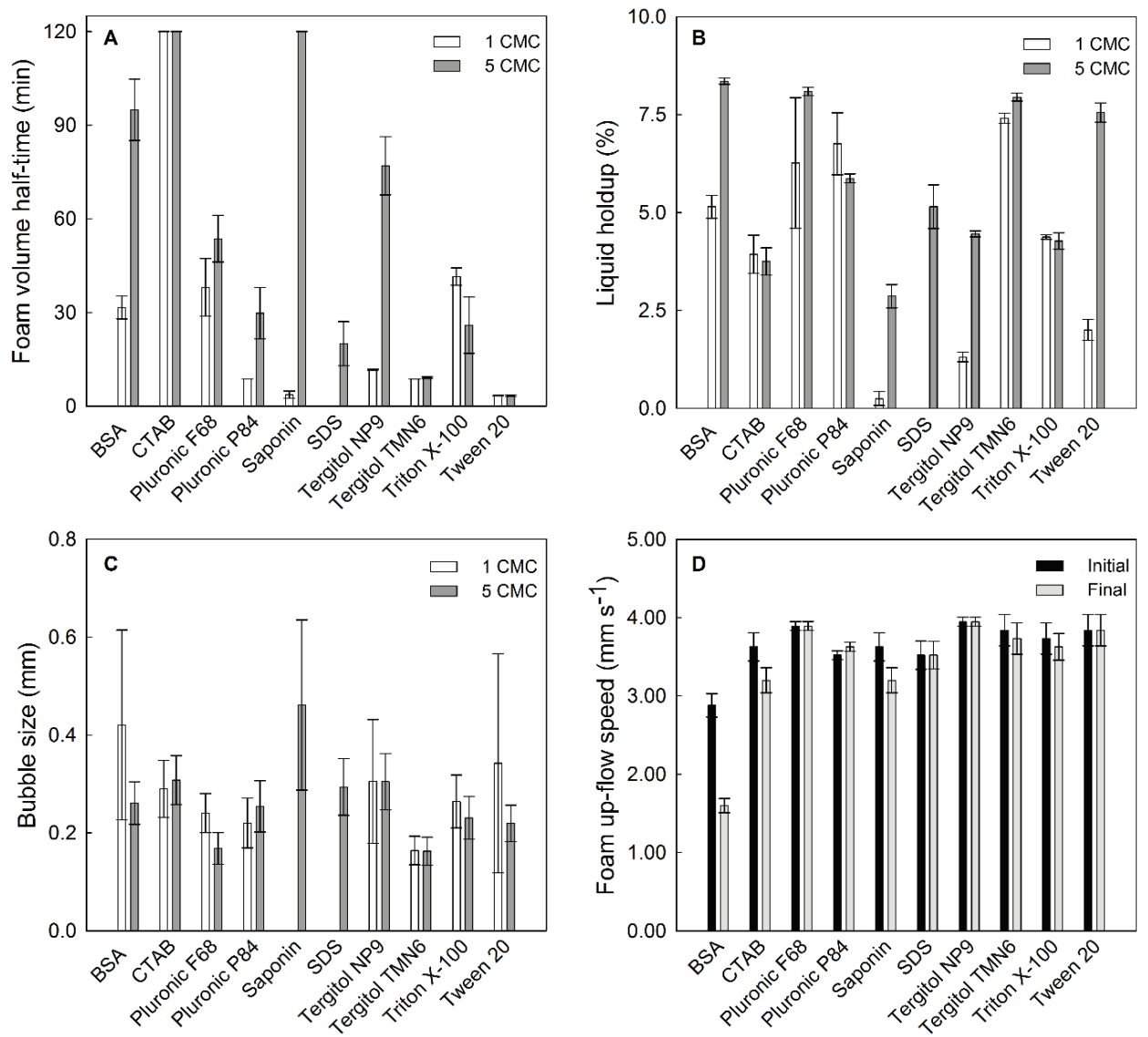

Figure 3.1. Surfactants foaming properties: A) Foam stability, represented in terms of foam volume half-life, which is the time elapsed when the foam volume has collapsed to its half. B) Liquid holdup in foams stabilized by different surfactants, expressed as the volume percentage of liquid incorporated in the foam. C) Bubble size of foams formed by different surfactants. D) Surfactants stability, represented as the initial and final foam up-flow speed of surfactant solutions after 20 cycles of repeated foam formation and deconstruction. Parameters $A, B$ and $C$ were analysed at two different surfactant concentrations (1 CMC and $5 \mathrm{CMC}$ ). SDS and Saponin at $1 \mathrm{CMC}$ did not form sufficiently stable foams for the analysis of bubble size. Liquid holdup and foam stability could not be measured with SDS at 1 $\mathrm{CMC}$ due to its low foaming ability. The error bars represent the standard deviation of the measurements.

\subsubsection{Surfactant biodegradability}

In order to select the most suitable surfactant, its susceptibility to bacterial degradation (i.e. biodegradation) was determined. This was done by following the growth of the inoculated bacteria (obtained from the supernatant of a S. obliquus culture) in the fresh water algal culture medium enriched with each surfactant at concentrations of 1 and $10 \mathrm{CMC}$. The surfactants added were the only reduced carbon source available to promote bacterial growth. Thus, 
bacterial growth, if observed, was a direct evidence for bacterial degradation of the surfactants as source of carbon and energy. Microalgal consumption of surfactants was not investigated since it was shown previously that BSA, one of the most easily biodegradable surfactants, was not consumed neither adsorbed by microalgal cells [6]. Therefore, the algal degradation of surfactants was considered negligible compared to bacterial degradation. Algal cells were removed from the inoculum, since decaying algal biomass due to the absence of light or surfactant toxicity might result in additional carbon source for bacterial growth.

Figure 3.2 shows results of bacterial growth on the different surfactants after 3 days of cultivation at 2 different concentrations (1 and $10 \mathrm{CMC})$. In order to strictly compare the surfactants in terms of the carbon content supplied, the X-axis is expressed as the decimal logarithm of the milligrams of carbon added to each culture, calculated from the carbon content of the surfactants. Cultures which showed bacterial growth reached the stationary phase and cultures that did not show any growth remained unchanged even after 2 weeks (data not shown). According to the figure, the lowest bacterial growth was obtained in the culture medium containing either CTAB, both Pluronics, or Triton X-100. For these surfactants no differences were observed for the two concentrations tested. Despite their high carbon content, compared to the other evaluated surfactants, the bacterial growth was minimal and, therefore, they can be considered less susceptible to biodegradation. On the other hand, the bacterial growth was by far more intense in the cultures containing BSA, Saponin and Tween 20 at 10 CMC, suggesting that indeed the carbon contained in these compounds was used as substrate for bacterial growth. A third group with surfactants which showed an intermediate biodegradability could be inferred from the figure. In this sense, both Tergitols and SDS promoted moderate bacterial growth. Bacterial growth was independent of the initial carbon content when SDS was used, which might indicate low efficiency of bacteria to degrade such surfactant and/or a possible toxic effect. Finally, regarding both Tergitols, a higher concentration of TMN6 was needed to promote the same bacterial growth as NP9, which might indicate Tergitol NP9 is slightly more readily biodegradable. 


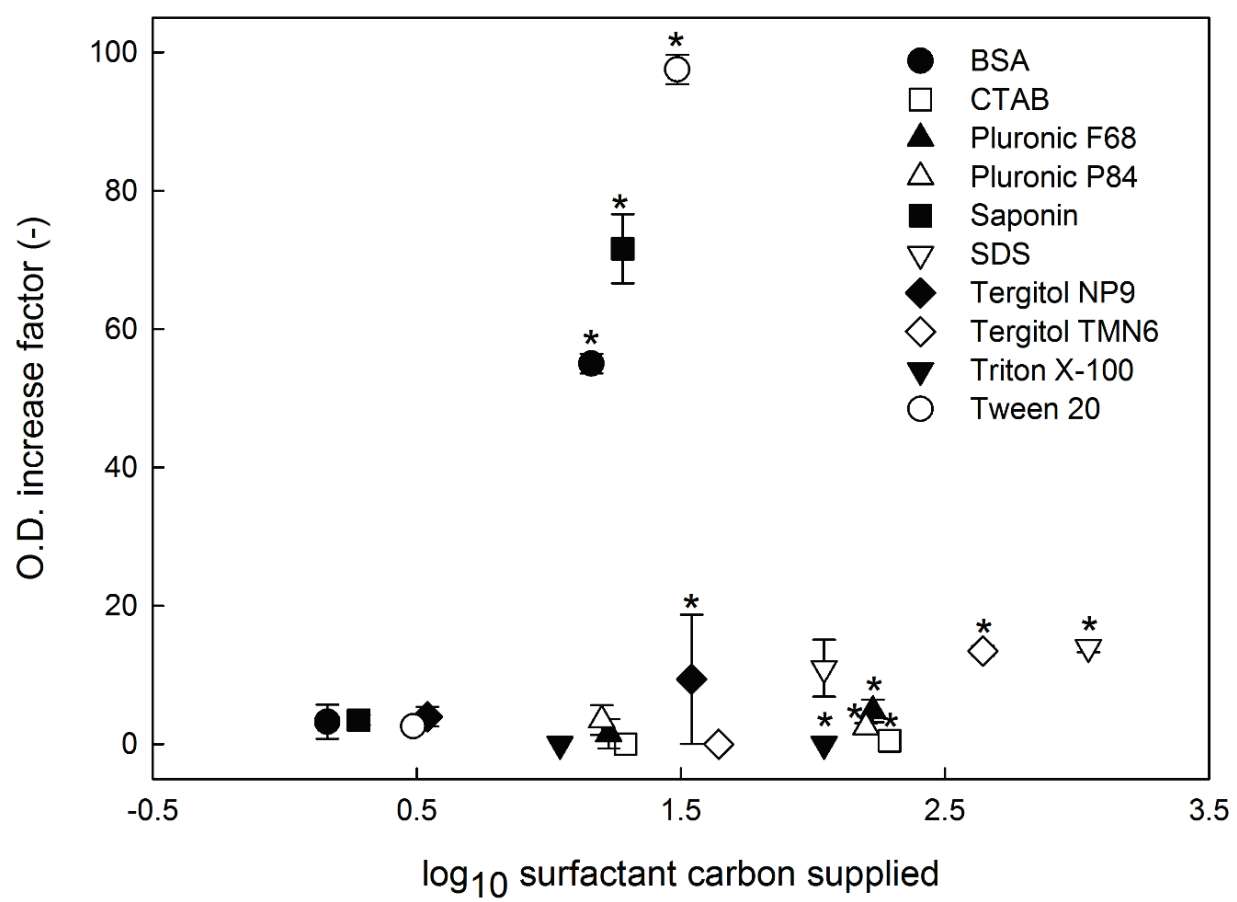

Figure 3.2. Surfactants biodegradability, represented as the bacterial growth on surfactant solutions after 3 days of cultivation at 1 and $10 \mathrm{CMC}$ surfactant concentrations. The "*" indicates data obtained at $10 \mathrm{CMC}$. Bacterial growth is expressed as the increase in optical density $(600 \mathrm{~nm})$ after 3 days and it is plotted against the carbon dosage resulting from surfactant addition (logarithmic values, in milligrams). Optical density values were corrected with their corresponding blanks (culture medium with surfactant) and optical density increments were normalized with respect to their initial optical densities.

The bacterial ability to degrade a surfactant and use it for growth depends on the chemical structure of the surfactant [9]. Three of the evaluated surfactants have fatty-alcohol ethoxylate structure (FAE; commercial names: Pluronic F68, Pluronic P84, Tergitol TMN6), and two of them have alkylphenolpolyethoxylate structure (APE; commercial names: Tergitol NP9 and Triton X-100). Under aerobic conditions, most surfactants can be degradable, including FAE and APE, and thus can favour bacterial growth [36]. However, there are differences between surfactant families regarding their degradability. For instance, according to the obtained results Triton X-100 (APE) is less readily biodegradable than SDS (alkylbenzenesulphonate), which is in agreement with previous observations [37]. The linear structure of SDS, compared to the branched and the ethoxylated chain of Triton X-100, may be among the reasons that explain its higher biodegradability [38]. 


\subsubsection{Microalgae partitioning towards the foam phase}

Microalgae partitioning to the foam phase, $P_{x}^{\text {foam }}$, has been measured and the results are presented in Figure 3.3. A partitioning of 1 means that the algae concentration in the foam liquid is identical to the initial concentration in the liquid prior foam formation, implying that it is also identical to that in underlying bulk liquid as can be calculated from a microalgae mass balance over the system. Detailed explanations about these mass balances are presented in Appendix 3.C. The first foam layer $(150 \mathrm{~mL})$ exiting the vessel showed large deviations in algae concentration compared to the following three foam fractions, thus it was not considered in the calculation of the $P_{x}^{\text {foam }}$ values. This deviation might be due to foam destabilisation at the top of the foam column and cells not draining efficiently through the foam layer as liquid drains $[39,40]$.

Only the cationic surfactant (CTAB) showed a partitioning higher than 1 . Microalgae cells concentrated in the CTAB stabilized foams, since the foam had 6.8 (C. sorokiniana) and 1.4 ( $S$. obliquus) times higher algae concentration compared to the original algae solution. This is reasoned by electrostatic interactions between cells and surfactants [41, 42], since cationic surfactants show a strong electrostatic interaction with the negatively charged microalgae cells $[14,43]$, as described for foam flotation systems. For all the other surfactants the partitioning factor was below 1 meaning that the algae have a preference to remain in the bulk liquid and not in the foam liquid. Despite the differences among those were not statistically significant, the natural surfactants (BSA and Saponin) and the non-ionic surfactant Triton X-100 showed slightly higher algae partitioning for the foam phase compared to the rest of the surfactants. The t-tests showed that these surfactants have significantly higher partitioning compared to most other surfactants, but not all. The 6 remaining surfactants ( 5 non-ionic, 1 anionic) showed similar $P_{x}^{\text {foam }}$ values. The anionic surfactant SDS did not result in the lowest partitioning for $C$. sorokiniana, however $S$. obliquus showed the lowest $P_{x}^{\text {foam }}$ results with SDS, possibly due to the electrostatic repulsion forces between the surfactant and the algal cell surface. As non-ionic surfactants do not own electrostatic charge, possibly hydrophobic interaction took place between the algal cell membranes and the surfactant [44]. Thus, the difference in algal partitioning between the different non-ionic surfactants might be due to their difference in hydrophobicity, affecting their efficiency of adsorption [42]. 


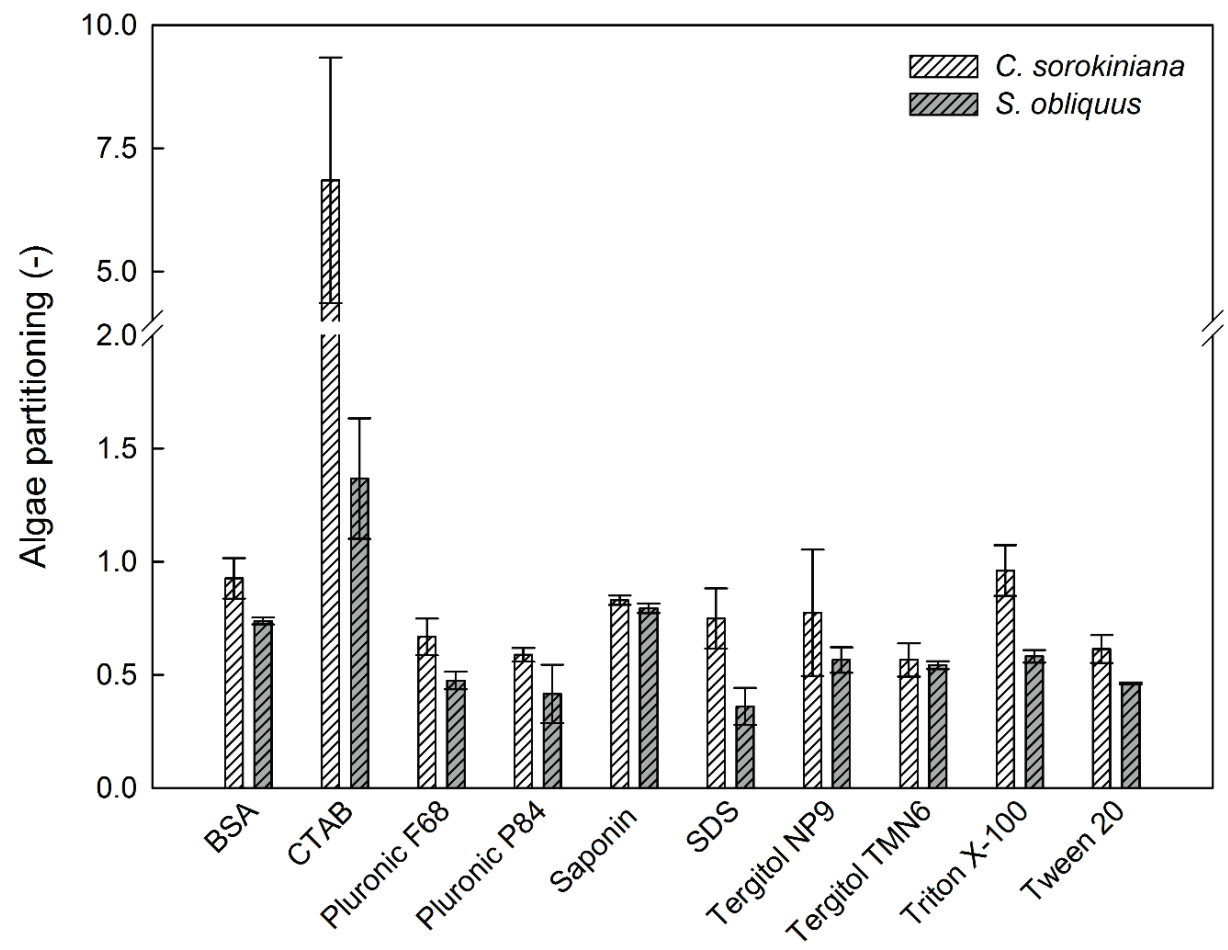

Figure 3.3. Chlorella sorokiniana (white hatched bars) and Scenedesmus obliquus (grey hatched bars) partitioning towards the foam phase. The partitioning is defined as the ratio between the algae concentration in the foam and the algae concentration of the suspension before foaming. The experiments were carried out at the surfactant concentration of $5 \mathrm{CMC}$ (except for Saponin where $5 \mathrm{CMC}$ did not generate enough foam for the measurements; thus, $10 \mathrm{CMC}$ was used). BSA was tested at a concentration of $1.75 \mathrm{~g} \mathrm{~L}^{-1}$. The microalgae concentration was 1520 expressed in $\mathrm{OD}$ at $750 \mathrm{~nm}$ for $\mathrm{C}$. sorokiniana and 2 for $\mathrm{S}$. obliquus.

In general, $C$. sorokiniana had a higher partitioning to the foam compared to $S$. obliquus. The differences in $P_{x}^{\text {foam }}$ between the two strains can be due to differences in the initial cell concentration applied, but also due to differences in cell surface hydrophobicity [45], since hydrophobic-hydrophilic interactions are determining the attachment of particles to air bubbles [46] and to surfactants [44]. Besides cell surface hydrophobicity, cell size and shape of the microalgae might have an influence on their partitioning: S. obliquus cells are long, narrow cells and larger in size than the small spherical C. sorokiniana cells.

\subsubsection{Toxicity of surfactants to microalgae}

The toxicity of the surfactants to Chlorella sorokiniana and Scenedesmus obliquus was studied by analyzing the effect of the surfactants on microalgal growth and photosynthetic efficiency 
(Figure 3.4). A surfactant concentration was considered toxic when a $10 \%$ reduction (dashed line in Figure 3.4), or higher, was found in optical density and/or photosynthetic efficiency with respect to the control cultures without surfactants. Although toxicity experiments were done at several different surfactant concentrations, Figure 3.4 represents only the points around the highest concentration without inhibitory effect and the lowest toxic concentration.

The toxicity results, considering the surfactant concentration used in weight per volume units, showed different sensitivity of each microalga to a given surfactant. In general, Scenedesmus obliquus cultures (Figure 3.4C, D) seem to be slightly less sensitive to evaluated surfactant concentrations than Chlorella sorokiniana (Figure 3.4A, B) as similar toxicity is produced at higher concentrations of most surfactants. The reason behind the species-dependent toxic effects of a certain surfactant might lay in the differences in algal cell wall composition between species [47]. Moreover, surfactants with different chemical structure, even owning the same electrostatic character (e.g. non-ionic), may exhibit different toxicity to a given microalga species. For instance, this is the case for both Pluronics, which exert different toxic effects on $S$. obliquus (Figure 3.4), Pluronic F68 being less toxic than Pluronic P84. Toxicity of poloxamers has been reported to be proportional to their lipophilic character [48], which is in agreement with our findings. One of the main reported effects of the surfactants is the cell surface depolarization [49] which has the consequence of decreasing nutrient consumption, therefore affecting algal growth negatively. The alterations of cell surface properties are related to the integration of alkyl chains into the cell wall [50]. 

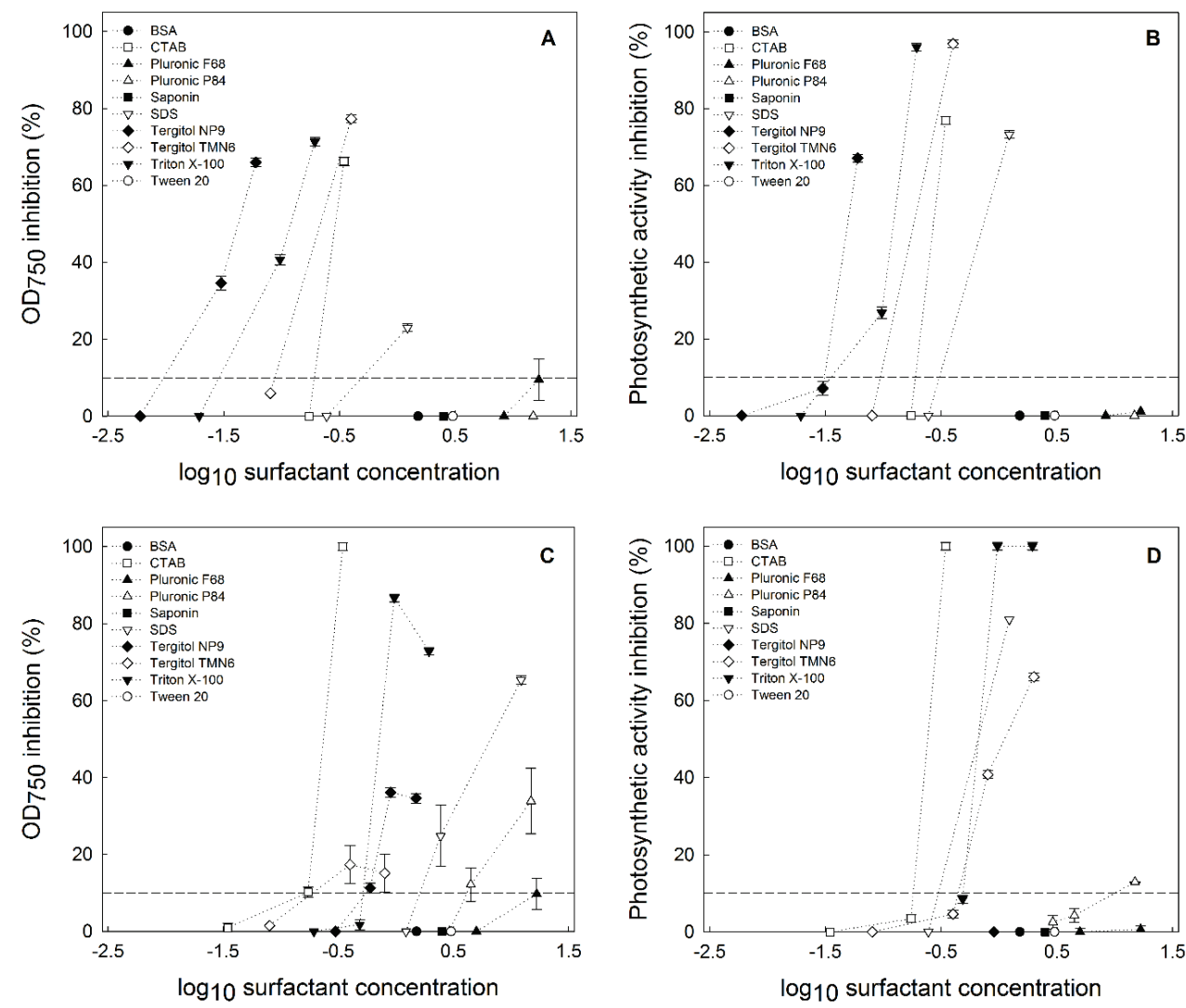

Figure 3.4. Surfactants concentration-response curves for C. sorokiniana (A-B) and S. obliquus (C-D) after 72 hours of exposure in liquid cultures. The ordinate indicates the percentage of inhibition in the biomass growth (represented as optical density at $750 \mathrm{~nm}$ ) (A, C), and in the maximum photosynthetic activity of Photosystem II (B, D) in dependence of the logarithm of the surfactant concentration used in grams per litre. The dashed line indicates the $10 \%$ inhibition threshold.

According to their toxic effects on the selected microalgae, the evaluated surfactants could be divided in two main groups. The two natural surfactants (BSA and Saponin), the two Pluronics (F68 and P84), and Tween 20 showed the lowest levels of toxicity to the tested microalgae species. On the opposite side, the ionic surfactants (CTAB and SDS), both Tergitols (NP9 and TMN6) and Triton X-100 exhibited the highest levels of toxicity to both microalgae. Altogether, it can be inferred that the toxicity of surfactants to microalgae depends on the specific chemical structure and concentration of the surfactant but it is also species-dependent $[36,49]$. 


\subsubsection{Surfactant selection}

In order to select the most appropriate surfactant for the liquid foam-bed photobioreactor, the foaming properties, biodegradability, microalgae partitioning to the foams, and toxicity have to be evaluated together.

The optimal surfactant for the liquid foam-bed photobioreactor should be capable of forming large foam volumes, with small and uniform bubbles. Small bubbles are relevant for increased mass transfer in the foam-bed photobioreactor due to the increased surface area. Further advantage of small bubbles is their increased particle flotation efficiency, possibly resulting in an increased algae partitioning towards the foam phase [51]. On the one hand, the formed foam should not be too stable ( $>2$ h, e.g. CTAB and Saponin at 5 CMC) in order to facilitate foam break-up. Fast destabilisation, on the other hand, as observed with Tween 20 and Tergitol TMN6, might result in foam collapse already while the foam is rising in the reactor, resulting in heterogeneous, coalescing foams. This would be especially problematic at low gas flow rates or high foam-bed photobioreactors. Besides, the liquid content of the foam should be intermediate in order to achieve increased biomass concentration compared to conventional photobioreactors, while still maintaining high ground-areal and volumetric productivities.

Altogether, the following ranges were identified for the different parameters defining the foaming properties: the liquid holdup of surfactants should be between $5-10 \%$, the foam volume half-life should be between 25 and 120 minutes, the bubble radius should be smaller than 0.4 $\mathrm{mm}$, and surfactants should show a constant foam up-flow speed during at least 20 cycles. As shown in Figure 3.1, surfactants with a higher liquid holdup than 5\% were BSA, Pluronic F68, Tergitol TMN6, Tween 20, Pluronic P84 and SDS at 5 CMC. Surfactants with intermediate foam stabilities were BSA, Tergitol NP9, Pluronic F68, Pluronic P84 and Triton X-100. The bubble size was too large for Saponin, all other surfactants fell in the good range. The protein BSA did not show sufficient stability against foaming.

Regarding surfactant biodegradability, the selected surfactant must not be easily degraded by the bacterial populations that are naturally associated to the algal culture, since the use of a readily biodegradable foam stabilising agent would imply a relative rapid increase of bacterial populations in the algal cultures, together with the need of a continuous addition of surfactant to maintain its concentration constant in the liquid foam-bed photobioreactor. In this study, 3 of the 10 surfactants tested (BSA, Saponin and Tween 20) were easily biodegraded at surfactant concentrations higher than $1 \mathrm{CMC}$. Considering the foam requirements, the surfactant 
concentration needs to be above $1 \mathrm{CMC}$ and, therefore, these surfactants are discarded for their use.

For the liquid foam-bed photobioreactor, maximal partitioning of microalgae to the foam phase is preferred. Elevated algae concentrations in the foam phase result in an increased fraction of algal cells exposed to illumination, therefore contributing to higher growth rates and reactor productivity. Due to the generally low algae partitioning of the non-ionic and anionic surfactants and the relatively small difference between their partitioning values, the cationic surfactant CTAB, Triton X-100, BSA and Saponin were considered to show best performances.

Last, but not least, a pre-requisite of a surfactant to be employed for algal cultivation in foam is to be non-toxic for the alga cultivated. In general, most of the surfactants showed toxicity below 10 CMC (Table 3.1). The cationic surfactant CTAB, the anionic surfactant (SDS), and both Tergitols resulted as the most toxic surfactants for both strains when comparing in terms of CMC. In this sense, the criterion for a non-toxic surfactant is the absence of toxic effects (no reduction of growth rate nor photosynthetic efficiency) at the concentration of $10 \mathrm{CMC}$. According to this, only both Pluronics and BSA, Saponin and Tween 20 fit the requirement. For the cultivation of other strains, the tolerance of the two species investigated to the given surfactants cannot be extrapolated to others and should be specifically studied.

Table 3.1. Experimental maximum non-toxic surfactant concentration tested for the microalgal strains $C$. sorokiniana and $\mathrm{S}$. obliquus, expressed in function of their $\mathrm{CMC}$ values. $\mathrm{CMC}$ values for the surfactants used in the experiments are included in Appendix 3.A.

\begin{tabular}{lllllllllll}
\hline \multirow{2}{*}{ Algal strain } & \multicolumn{10}{c}{ Experimental maximum non-toxic surfactant concentration (CMC) } \\
\cline { 2 - 11 } & BSA & CTAB & $\begin{array}{l}\text { Pluronic } \\
\text { F68 }\end{array}$ & $\begin{array}{l}\text { Pluronic } \\
\text { P84 }\end{array}$ & Saponin & SDS & $\begin{array}{l}\text { Tergitol } \\
\text { NP9 }\end{array}$ & Tergitol & Triton & Tween \\
TM-100 & 20 \\
\hline C. sorokiniana & 50 & 0.5 & 50 & 50 & 50 & 0.1 & 0.1 & 0.1 & 0.1 & 50 \\
S. obliquus & 50 & 0.1 & 50 & 10 & 50 & 0.1 & 5 & 0.1 & 2.5 & 50 \\
\hline
\end{tabular}

Surfactants showing acceptable properties with respect to each individual criterion were indicated with a '+' in Table 3.2. Considering all properties together, the poloxameric surfactants are the best candidates investigated for the operation of the liquid foam-bed photobioreactor, due to their good foaming properties, resistance to biodegradation, moderate algae partitioning, and low toxicity. The most important limitation of these surfactants lies in the fact that they have low $P_{x}^{\text {foam }}$ values (i.e. partioning towards the foam). By minimizing the amount of bulk liquid in an operational foam-bed photobioreactor, the absolute amount of algae 
in the relatively dark bulk liquid can be minimized and the majority of the microalgae still reside in the foam phase. Furthermore, the algae partitioning towards the foam formed by poloxamers can be further improved in the foam-bed photobioreactor by increased surfactant concentration [41], increased aeration rates [14], and reduced bubble sizes [39, 52, 53]. Alternatively, the microalgae-enriched bulk liquid phase can be recirculated and distributed on the top of the foam column. From the two tested poloxamers, Pluronic F68 was selected as the best surfactant since it performed slightly better in terms of foaming, algae partitioning and toxicity.

The Foam-bed photobioreactor is a promising photobioreactor concept with several advantages over liquid-phase based cultivation systems. Foam-bed photobioreactors can offer reduced light absorption path and increased mass transfer rates compared to traditional photobioreactors. Surfactant selection is a key factor in the success of the operation. Pluronic F68 appeared to be a surfactant which can allow for long-term operation of foam-bed photobioreactors. High biomass densities and reduced gas flow rates and pressured drops in foam-bed photobioreactors will then contribute to a reduction in biomass production costs.

Table 3.2. Summary of the surfactant selection experiments. The "t" indicates that a certain surfactant has acceptable properties regarding the corresponding criterion. Foaming properties: foam volume half-life time (A), liquid holdup in the foam (B), bubble size of the foam (C) and surfactant stability (D).

\begin{tabular}{|c|c|c|c|c|c|c|c|}
\hline & \multicolumn{4}{|c|}{ Foaming properties } & \multirow{2}{*}{ Algae partitioning } & \multirow{2}{*}{$\begin{array}{l}\text { Resistance to } \\
\text { biodegradability }\end{array}$} & \multirow{2}{*}{ Toxicity } \\
\hline & A & B & C & D & & & \\
\hline BSA & + & + & + & - & + & - & + \\
\hline CTAB & - & - & + & + & + & + & - \\
\hline Pluronic F68 & + & + & + & + & - & + & + \\
\hline Pluronic P84 & + & + & + & + & - & + & + \\
\hline Saponin & - & - & - & + & + & - & + \\
\hline SDS & - & + & + & + & - & - & - \\
\hline Tergitol NP9 & + & - & + & + & - & - & - \\
\hline Tergitol TMN6 & - & + & + & + & - & - & - \\
\hline Triton X-100 & + & - & + & + & + & + & - \\
\hline Tween 20 & - & - & + & + & - & - & + \\
\hline
\end{tabular}

\subsection{Conclusions}

This project was undertaken to select the best foam stabilising agent to be applied in a liquid foam-bed photobioreactor. First a pre-selection was made of 10 potential candidates which were tested in terms of their foaming properties, biodegradability, microalgae partitioning towards 
the foam phase, and toxicity. According to the experimentally obtained data, poloxameric surfactants (Pluronic F68 and Pluronic P84) exhibited the best properties and Pluronic F68 was selected as the best surfactant because of its slightly better performance. Pluronic F68 showed constant foamability during repetitive foaming cycles and at $5 \mathrm{CMC}$ concentration it formed foams with intermediate stability (54 minutes half-life), high liquid holdup ( $8 \%$ at $400 \mathrm{~mL} \mathrm{~min}{ }^{-}$ ${ }^{1}$ superficial gas velocity), and small bubble size (radius of $0.17 \mathrm{~mm}$ ) which are optimal for the operation of the liquid foam-bed photobioreactor. Besides, it is hardly biodegradable by the bacteria naturally-associated to microalgae, therefore the surfactant concentration in the reactor is not reduced due to bacterial consumption. The relatively low microalgae partitioning towards the foam phase formed by Pluronic F68 (67\% for C. sorokiniana and 47\% for S. obliquus) can be compensated by optimizing operation and design of a liquid foam-bed photobioreactor. For example, foam-bed photobioreactor systems where the microalgae-enriched liquid layer is recirculated over the foam phase could result in an economically feasible algae production system. Finally, growth inhibition was not observed for any of the microalgal strains tested until 50 CMC. Thus, Pluronic F68 is a promising, new foam stabilising agent for a liquid foam-bed photobioreactor. 


\section{Appendix 3.A: Summary of foam stabilizing agents}

Table 3.A.1. Summary of the foam stabilizing agents used in this study. P.I. stands for the online product information sheets. The superscript ' $a$ ' indicates the CMC value used for the foaming and partitioning experiments, while ' $b$ ' indicates the values used for the biodegradability and toxicity experiments.

\begin{tabular}{|c|c|c|c|c|c|}
\hline $\begin{array}{l}\text { Foam stabilizing } \\
\text { agent }\end{array}$ & $\begin{array}{l}\text { Source } \\
\text { details }\end{array}$ & Type & $\mathrm{CMC}\left[\mathrm{g} \mathrm{L}^{-1}\right]$ & Ref. CMC & Chemical composition \\
\hline BSA & $\begin{array}{l}\text { Sigma, } \\
\text { A7030 }\end{array}$ & $\begin{array}{l}\text { Non- } \\
\text { catalytic } \\
\text { protein, } \\
\text { anionic } \\
\text { character }\end{array}$ & 0.03 & {$[35], 25^{\circ} \mathrm{C}$} & $\begin{array}{l}\text { Bovine Serum Albumin } \\
\text { derived from cows }\end{array}$ \\
\hline CTAB & $\begin{array}{l}\text { Sigma } \\
\mathrm{H} 5882\end{array}$ & Cationic & $\begin{array}{l}0.335^{a} \\
0.346^{b}\end{array}$ & $\begin{array}{l}\text { Sigma P.I., 20- } \\
25^{\circ} \mathrm{C} \text { a, } \\
\text { minimum given } \\
\text { value } \\
\text { Sigma P.I., 20- } \\
25^{\circ} \mathrm{C}^{b} \text {, middle } \\
\text { of given range }\end{array}$ & $\begin{array}{l}\text { Hexadecyltrimethylammonium } \\
\text { bromide }\end{array}$ \\
\hline Pluronic F68 & $\begin{array}{l}\text { Panreac } \\
\text { A1288 }\end{array}$ & Non-ionic & 0.334 & $\begin{array}{l}\text { Sigma Aldrich } \\
\text { P.I. }\end{array}$ & $\begin{array}{l}\text { Triblock copolymers of } \\
\text { ethylene and propylene oxide }\end{array}$ \\
\hline Pluronic P84 & $\begin{array}{l}\text { Sigma } \\
713538\end{array}$ & Non-ionic & 0.298 & [54] & $\begin{array}{l}\text { Triblock copolymers of } \\
\text { ethylene and propylene oxide }\end{array}$ \\
\hline Saponin & $\begin{array}{l}\text { Sigma } \\
84510\end{array}$ & Non-ionic & 0.05 & $\begin{array}{l}\text { Sigma P.I., } \\
\text { middle of given } \\
\text { range }\end{array}$ & Glycoside+ Triterpene \\
\hline SDS & $\begin{array}{l}\text { Sigma } \\
\text { L3771 }\end{array}$ & Anionic & 2.451 & $\begin{array}{l}\text { Sigma P.I., } \\
\text { middle of given } \\
\text { range } 20-25^{\circ} \mathrm{C}\end{array}$ & Sodium dodecyl sulfate \\
\hline Tergitol NP9 & $\begin{array}{l}\text { Sigma } \\
521078\end{array}$ & Non-ionic & 0.06 & $\begin{array}{l}\text { DOW P.I., } \\
25^{\circ} \mathrm{C}\end{array}$ & Nonylphenolethoxylate \\
\hline Tergitol TMN6 & $\begin{array}{l}\text { Sigma } \\
60828\end{array}$ & Non-ionic & 0.8 & $\begin{array}{l}\text { DOW P.I., } \\
25^{\circ} \mathrm{C}\end{array}$ & $\begin{array}{l}\text { Branched secondary alcohol } \\
\text { ethoxylate }\end{array}$ \\
\hline Triton X-100 & $\begin{array}{l}\text { Panreac } \\
\text { A1388 }\end{array}$ & Non-ionic & $\begin{array}{l}0.155^{a} \\
0.194^{b}\end{array}$ & $\begin{array}{l}{[55]^{a}} \\
\text { Applichem P.I. }{ }^{\text {b }}\end{array}$ & Octyl phenol ethoxylate \\
\hline Tween 20 & $\begin{array}{l}\text { Sigma, } \\
\text { P2287a } \\
\text { Sigma, } \\
\text { P1379 b }\end{array}$ & Non-ionic & $0.099 a$ & $\begin{array}{l}{[56], 21^{\circ} \mathrm{Ca}} \\
{[57], \text { room }^{\circ}} \\
\text { temperature }^{\mathrm{b}}\end{array}$ & $\begin{array}{l}\text { Polyoxyethylene } \\
\text { sorbitanmonolaurate }\end{array}$ \\
\hline
\end{tabular}




\section{Appendix 3.B: Medium recipes}

\section{B.1 Modified Breuer's medium}

The recipe used in this study contains $\mathrm{KNO}_{3} 2.55 \mathrm{~g} \mathrm{~L}^{-1}, \mathrm{Na}_{2} \mathrm{SO}_{4} 0.15 \mathrm{~g} \mathrm{~L}^{-1}, \mathrm{MgSO}_{4} \cdot 7 \mathrm{H}_{2} \mathrm{O} 0.22$ $\mathrm{g} \mathrm{L}^{-1}, \mathrm{CaCl}_{2} \cdot 2 \mathrm{H}_{2} \mathrm{O} 0.11 \mathrm{~g} \mathrm{~L}^{-1}, \mathrm{~K}_{2} \mathrm{HPO}_{4} 0.645 \mathrm{~g} \mathrm{~L}^{-1}, \mathrm{Na}_{2}$-EDTA $2 \mathrm{H}_{2} \mathrm{O} 44.64 \mathrm{mg} \mathrm{L} \mathrm{L}^{-1}$, $\mathrm{MnCl}_{2} \cdot 4 \mathrm{H}_{2} 05.72 \mathrm{mg} \mathrm{L}^{-1}, \mathrm{ZnSO}_{4} \cdot 7 \mathrm{H}_{2} \mathrm{O} 1.8 \mathrm{mg} \mathrm{L}^{-1}, \mathrm{CoCl}_{2} \cdot 6 \mathrm{H}_{2} \mathrm{O} 0.423 \mathrm{mg} \mathrm{L}^{-1}, \mathrm{CuSO}_{4} \cdot 5 \mathrm{H}_{2} \mathrm{O}$ $0.495 \mathrm{mg} \mathrm{L}^{-1}, \mathrm{Na}_{2} \mathrm{MoO}_{4} \cdot 2 \mathrm{H}_{2} \mathrm{O} 0.0378 \mathrm{mg} \mathrm{L}^{-1}$ and NaFeEDTA $15.3 \mathrm{mg} \mathrm{L}^{-1}$. The $\mathrm{pH}$ of the media was set to 7.0 .

\section{B.2 Modified M8a medium (algae partitioning)}

The media contained the following nutrients: urea $3.60 \mathrm{~g} \mathrm{~L}^{-1}, \mathrm{KH}_{2} \mathrm{PO}_{4} 1.07 \mathrm{~g} \mathrm{~L}^{-1}$, $\mathrm{Na}_{2} \mathrm{HPO}_{4} .2 \mathrm{H}_{2} \mathrm{O} 0.37 \mathrm{~g} \mathrm{~L}^{-1}, \mathrm{MgSO}_{4} \cdot 7 \mathrm{H}_{2} \mathrm{O} 1.2 \mathrm{~g} \mathrm{~L}^{-1}, \mathrm{CaCl}_{2} \cdot 2 \mathrm{H}_{2} \mathrm{O} 39 \mathrm{mg} \mathrm{L}{ }^{-1}$, EDTA ferric sodium salt $347.8 \mathrm{mg} \mathrm{L}^{-1}, \mathrm{Na}_{2} \mathrm{EDTA} \cdot 2 \mathrm{H}_{2} \mathrm{O} 111.67 \mathrm{mg} \mathrm{L}^{-1}, \mathrm{H}_{3} \mathrm{BO}_{3} 0.185 \mathrm{mg} \mathrm{L}^{-1}, \mathrm{MnCl}_{2} \cdot 4 \mathrm{H}_{2} \mathrm{O}$ $38.9 \mathrm{mg} \mathrm{L}^{-1}, \mathrm{ZnSO}_{4} \cdot 7 \mathrm{H}_{2} \mathrm{O} 9.6 \mathrm{mg} \mathrm{L}^{-1}, \mathrm{CuSO}_{4} \cdot 5 \mathrm{H}_{2} \mathrm{O} 5.49 \mathrm{~L}^{-1}$. After the addition of all nutrients, the $\mathrm{pH}$ was adjusted with $\mathrm{NaOH}$ to $\mathrm{pH} 6.7$. 


\section{Appendix 3.C: Mass balances microalgae partitioning}

Mass balances for the microalgae cells over the foam-bed system were set up in order to explain in detail the microalgae partitioning coefficient and the factors influencing that. These equations correspond to the experimental setup of the microalgae partitioning experiments performed. A scheme of the experimental setup for microalgae partitioning is presented in Figure 3.C.1.

Equation 3.C.1 describes that the cells present in the initial liquid prior to foam formation will be distributed over the bulk liquid and the liquid fraction of the foam after foam formation. Equation 3.C.2 explains that also the initial liquid volume will be distributed over the bulk liquid phase and the foam phase after foam formation. The notation corresponding to these equations is presented in Table 3.C.1. The partition coefficient (Equation 3.C.3) results in exactly 1 when there is no preference for the algal cells for either the foam phase or bulk liquid phase. $P_{x}^{\text {foam }}$ values above, or below 1 , give a relative indication of the level of preference of algal cells for either phase.

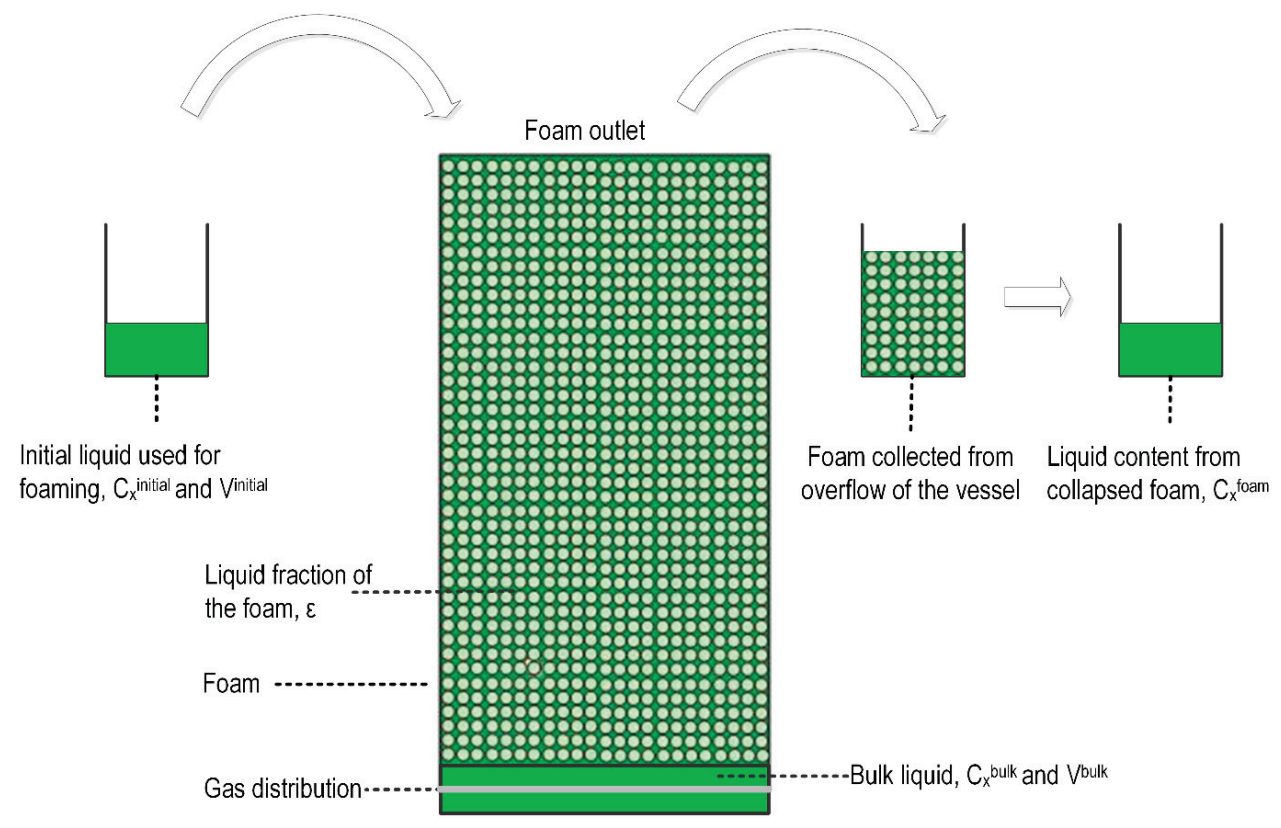

Figure 3.C.1. Scheme of the experimental procedure for microalgae partitioning measurements.

$V^{\text {initial }} \cdot C_{x}^{\text {initial }}=C_{x}^{\text {bulk }}(t) \cdot V^{\text {bulk }}(t)+V^{\text {total }}(t) \cdot \varepsilon_{\text {avg }}(t) \cdot C_{\text {xavg }}^{\text {foam }}(t)$

Equation 3.C.1 


$$
\begin{array}{ll}
V^{\text {initial }}=V^{\text {bulk }}(t)+V^{\text {total }}(t) \cdot \varepsilon_{\text {avg }}(t) & \text { Equation 3.C.2 } \\
P_{x}^{\text {foam }}=\frac{C_{x}^{\text {foam }}\left(t=t_{\text {test }}\right)}{C_{x}^{\text {initial }}} & \text { Equation 3.C.3 }
\end{array}
$$

The algae partitioning experiments were done in a batch system and therefore the bulk liquid volume, bulk liquid algae concentration and bulk liquid surfactant concentration might change in time with increasing foam volume. In addition, the liquid fraction of foams decreases in height due to gravitational drainage in the foam. Consequently, in the equations the average liquid fraction along the foam height has to be considered. The biomass concentration in the foam is therefore also not homogenously distributed in the different foam fractions.

The dynamics of the systems discussed above did not influence our measurements. We collected less than $20 \%$ of the total liquid volume which was initially added and no trends were observed between the different foam fractions collected in time. Changes in the partitioning apparently were not observable within the experimental time and foam volumes sampled.

To avoid any influences of experimental conditions on $P_{x}^{\text {foam }}$, the same protocol was used for each measurement. This includes a fixed foam volume collected, a fixed total foam volume and therefore fixed foam formation time, a fixed gas flow rate, initial liquid volume, initial algae concentration, and surfactant concentration. In this way the different surfactants can be appropriately compared in terms of algal partitioning as defined with Equation 3.C.3.

\begin{tabular}{|c|c|}
\hline Symbol & Explanation \\
\hline $\bar{\varepsilon}$ & $\begin{array}{l}\text { local liquid fraction in the foam, thus the liquid volume incorporated in the foam divided by } \\
\text { the foam volume }\end{array}$ \\
\hline$\varepsilon_{\text {avg }}$ & average liquid fraction in the foam \\
\hline$C_{x}$ initial & biomass concentration in the initial solution, before foam formation \\
\hline$C_{x}^{\text {bulk }}$ & biomass concentration in the bulk liquid phase \\
\hline$C_{x}^{\text {foam }}$ & local biomass concentration in the liquid fraction of the foam \\
\hline$C_{x, a v g}^{\text {foam }}$ & average biomass concentration in the liquid fraction of the foam \\
\hline Vbulk & bulk liquid volume (liquid layer underneath the foam) \\
\hline Vital & total foam volume \\
\hline Vinitial & total liquid volume of the foaming solution, measured prior to foam formation \\
\hline
\end{tabular}

Table 3.C.1. Notation related to the mass balances. 


\section{References}

[1] J. Milledge, Commercial application of microalgae other than as biofuels: a brief review, Rev. Environ. Sci. 10 (2011) 31-41.

[2] J. Ruiz, G. Olivieri, J. de Vree, R. Bosma, P. Willems, H. Reith, M. Eppink, D.M. Kleinegris, R.H. Wijffels, M. Barbosa, Towards industrial products from microalgae, Energy Environ. Sci. 9 (2016) 3036-3043.

[3] E.P. Resurreccion, L.M. Colosi, M.A. White, A.F. Clarens, Comparison of algae cultivation methods for bioenergy production using a combined life cycle assessment and life cycle costing approach, Bioresour. Technol. 126 (2012) 298-306.

[4] F.G. Acién, J.M. Fernández, J.J. Magán, E. Molina, Production cost of a real microalgae production plant and strategies to reduce it, Biotechnol. Adv. 30 (2012) 1344-1353.

[5] C. Posten, Design principles of photo-bioreactors for cultivation of microalgae, Eng. Life Sci. 9 (2009) 165-177.

[6] A. Janoska, P.P. Lamers, A. Hamhuis, Y. van Eimeren, R.H. Wijffels, M. Janssen, A liquid foam-bed photobioreactor for microalgae production, Chem. Eng. J. 313 (2017) 1206-1214.

[7] D. Beneventi, B. Carre, A. Gandini, Role of surfactant structure on surface and foaming properties, Colloids Surf., A 189 (2001) 65-73.

[8] E. Jurado, M. Fernández-Serrano, F. Ríos, M. Lechuga, Aerobic biodegradation of surfactants, in R. Chamy and F. Rosenkranz (Eds.), Biodegradation-life of science, Intech, Rijeka, 2013, pp. 63-81.

[9] M.J. Scott, M.N. Jones, The biodegradation of surfactants in the environment, Biochim. Biophys. Acta, Biomembr. 1508 (2000) 235-251.

[10] P.B. Dorn, J.P. Salanitro, S.H. Evans, L. Kravetz, Assessing the aquatic hazard of some branched and linear nonionic surfactants by biodegradation and toxicity, Environ. Toxicol. Chem. 12 (1993) 1751-1762.

[11] H. Nyberg, Growth of Selenastrum capricornutum in the presence of synthetic surfactants, Water Res. 22 (1988) 217-223.

[12] J.L. Fuentes, I. Garbayo, M. Cuaresma, Z. Montero, M. González-del-Valle, C. Vílchez, Impact of microalgae-bacteria interactions on the production of algal biomass and associated compounds, Mar. Drugs 14 (2016) 100.

[13] S. Garg, L. Wang, P.M. Schenk, Effects of algal hydrophobicity and bubble size on flotation separation of microalgae from aqueous medium [online], In: Chemeca 2013 (41st : 2013 : Brisbane, Qld.). Chemeca 2013: Challenging Tomorrow. Barton, ACT: Engineers Australia, 2013, 673-677.

[14] W. Phoochinda, D. White, Removal of algae using froth flotation, Environ. Technol. 24 (2003) 87-96.

[15] R.K. Henderson, S.A. Parsons, B. Jefferson, The potential for using bubble modification chemicals in dissolved air flotation for algae removal, Sep. Sci. Technol. 44 (2009) 1923-1940.

[16] A. Azizullah, P. Richter, D.-P. Häder, Photosynthesis and photosynthetic pigments in the flagellate Euglena gracilis-As sensitive endpoints for toxicity evaluation of liquid detergents, J. Photochem. Photobiol., B 133 (2014) 18-26.

[17] A. Azizullah, P. Richter, M. Jamil, D.-P. Häder, Chronic toxicity of a laundry detergent to the freshwater flagellate Euglena gracilis, Ecotoxicology 21 (2012) 1957-1964.

[18] M. Garrido-Perez, J. Perales-Vargas Machuca, E. Nebot-Sanz, D. Sales-Márquez, Effect of the test media and toxicity of LAS on the growth of Isochrysis galbana, Ecotoxicology 17 (2008) 738-746.

[19] V. Maksimov, T. Parshikova, Influence of surfactants on the photosynthetic activity of algae, Hydrobiol. J. 42 (2006) 67-76.

[20] Ž. Pavlić, Ž. Vidaković-Cifrek, D. Puntarić, Toxicity of surfactants to green microalgae Pseudokirchneriella subcapitata and Scenedesmus subspicatus and to marine diatoms Phaeodactylum tricornutum and Skeletonema costatum, Chemosphere 61 (2005) 1061-1068.

[21] M. Lewis, Chronic toxicities of surfactants and detergent builders to algae: a review and risk assessment, Ecotoxicol. Environ. Saf. 20 (1990) 123-140.

[22] M. Cuaresma, M. Janssen, C. Vilchez, R.H. Wijfels, Productivity of Chlorella sorokiniana in a short light-path (SLP) panel photobioreactor under high irradiance, Biotechnol. Bioeng. 104 (2009) 352-359.

[23] E. Barbera, E. Sforza, A. Bertucco, Maximizing the production of Scenedesmus obliquus in photobioreactors under different irradiation regimes: experiments and modeling, Bioprocess Biosyst. Eng. 38 (2015) 2177-2188.

[24] A.M. Kliphuis, L. de Winter, C. Vejrazka, D.E. Martens, M. Janssen, R.H. Wijffels, Photosynthetic efficiency of Chlorella sorokiniana in a turbulently mixed short light-path photobioreactor, Biotechnol. Prog. 26 (2010) 687-696. 
[25] G. Breuer, L. de Jaeger, V.P. Artus, D.E. Martens, J. Springer, R.B. Draaisma, G. Eggink, R.H. Wijffels, P.P. Lamers, Superior triacylglycerol (TAG) accumulation in starchless mutants of Scenedesmus obliquus:(II) evaluation of TAG yield and productivity in controlled photobioreactors, Biotechnol. Biofuels 7 (2014) 70.

[26] R. Bosma, K. Miazek, S. Willemsen, M. Vermuë, R. Wijffels, Growth inhibition of Monodus subterraneus by free fatty acids, Biotechnol. Bioeng. 101 (2008) 1108-1114.

[27] S. Boonyasuwat, S. Chavadej, P. Malakul, J.F. Scamehorn, Surfactant recovery from water using a multistage foam fractionator: Part I effects of air flow rate, foam height, feed flow rate and number of stages, Sep. Sci. Technol. 40 (2005) 1835-1853.

[28] M. Rosen, J. Solash, Factors affecting initial foam height in the Ross-Miles foam test, J. Am. Oil Chem. Soc. 46 (1969) 399-402.

[29] J. Boos, W. Drenckhan, C. Stubenrauch, On how surfactant depletion during foam generation influences foam properties, Langmuir 28 (2012) 9303-9310.

[30] E. Carey, C. Stubenrauch, Properties of aqueous foams stabilized by dodecyltrimethylammonium bromide, J. Colloid Interface Sci. 333 (2009) 619-627.

[31] D. Beneventi, R.J. Pugh, B. Carré, A. Gandini, Surface rheology and foaming properties of sodium oleate and C 12 (EO) 6 aqueous solutions, J. Colloid Interface Sci. 268 (2003) 221-229.

[32] P. Stevenson, Hydrodynamic theory of rising foam, Miner. Eng. 20 (2007) 282-289.

[33] R. Lemlich, Prediction of changes in bubble size distribution due to interbubble gas diffusion in foam, Ind.

Eng. Chem. Fundam. 17 (1978) 89-93.

[34] N.S. Deshpande, M. Barigou, Performance characteristics of novel mechanical foam breakers in a stirred tank reactor, J. Chem. Technol. Biotechnol. 74 (1999) 979-987.

[35] J. Clarkson, Z. Cui, R. Darton, Protein denaturation in foam: II. Surface activity and conformational change, J. Colloid Interface Sci. 215 (1999) 333-338.

[36] M. Lechuga, M. Fernández-Serrano, E. Jurado, J. Núñez-Olea, F. Ríos, Acute toxicity of anionic and nonionic surfactants to aquatic organisms, Ecotoxicol. Environ. Saf. 125 (2016) 1-8.

[37] G.C. Okpokwasili and A.O. Olisa, River-water biodegradation of surfactants in liquid detergents and shampoos, Water Res. 25 (1991) 1425-1429.

[38] G. Zeng, H. Fu, H. Zhong, X. Yuan, M. Fu, W. Wang, G. Huang, Co-degradation with glucose of four surfactants, CTAB, Triton X-100, SDS and Rhamnolipid, in liquid culture media and compost matrix, Biodegradation 18 (2007) 303-310.

[39] W. Hu, R. Gladue, J. Hansen, C. Wojnar, J.J. Chalmers, The Sensitivity of the Dinoflagellate Crypthecodinium cohnii to Transient Hydrodynamic Forces and Cell-Bubble Interactions, Biotechnol. Prog. 23 (2007) 1355-1362.

[40] P.E. Wiley, K.J. Brenneman, A.E. Jacobson, Improved algal harvesting using suspended air flotation, Water Environ. Res. 81 (2009) 702-708.

[41] Y.M. Chen, J.C. Liu, Y.-H. Ju, Flotation removal of algae from water, Colloids Surf., B. 12 (1998) 49-55.

[42] R.K. Henderson, S.A. Parsons, B. Jefferson, Surfactants as bubble surface modifiers in the flotation of algae: dissolved air flotation that utilizes a chemically modified bubble surface, Environ. Sci. Technol. 42 (2008) 48834888.

[43] W. Phoochinda, D. White, B. Briscoe, An algal removal using a combination of flocculation and flotation processes, Environ. Technol. 25 (2004) 1385-1395.

[44] M.J. Rosen, F. Li, S.W. Morrall, D.J. Versteeg, The relationship between the interfacial properties of surfactants and their toxicity to aquatic organisms, Environ. Sci. Technol. 35 (2001) 954-959.

[45] S. Garg, Y. Li, L. Wang, P.M. Schenk, Flotation of marine microalgae: effect of algal hydrophobicity, Bioresour. Technol. 121 (2012) 471-474.

[46] W.A. Ducker, Z. Xu, J.N. Israelachvili, Measurements of hydrophobic and DLVO forces in bubble-surface interactions in aqueous solutions, Langmuir 10 (1994) 3279-3289.

[47] G. Corre, J. Templier, C. Largeau, B. Rousseau, C. Berkaloff, Influence of cell wall composition on the resistance of two Chlorella species (Chlorophyta) to detergents, J. Phycol. 32 (1996) 584-590.

[48] T.P. Johnston, S.C. Miller, Toxicological evaluation of poloxamer vehicles for intramuscular use, PDA J. Pharm. Sci. Technol. 39 (1985) 83-89.

[49] S. Rebello, A.K. Asok, S. Mundayoor, M. Jisha, Surfactants: chemistry, toxicity and remediation, In: E. Lichtfouse, J. Schwarzbauer, D. Robert (Eds.), Pollutant Diseases, Remediation and Recycling, Springer,

Switzerland, 2013, pp. 277-320. 
[50] B. Yoo, B. Jing, S.E. Jones, G.A. Lamberti, Y. Zhu, J.K. Shah, E.J. Maginn, Molecular mechanisms of ionic liquid cytotoxicity probed by an integrated experimental and computational approach, Sci. Rep. 6 (2016) 19889. [51] A. Al-Shamrani, A. James, H. Xiao, Separation of oil from water by dissolved air flotation, Colloids Surf., A 209 (2002) 15-26.

[52] T.N. Hunter, R.J. Pugh, G.V. Franks, G.J. Jameson, The role of particles in stabilising foams and emulsions, Adv. Colloid Interface Sci. 137 (2008) 57-81.

[53] T.M. Sobczuk, F.G. Camacho, E.M. Grima, Y. Chisti, Effects of agitation on the microalgae Phaeodactylum tricornutum and Porphyridium cruentum, Bioprocess Biosyst. Eng. 28 (2006) 243-250.

[54] M.Y. Kozlov, N.S. Melik-Nubarov, E.V. Batrakova, A.V. Kabanov, Relationship between pluronic block copolymer structure, critical micellization concentration and partitioning coefficients of low molecular mass solutes. Macromolecules 33 (2000) 3305-3313.

[55] S.H. Chang, K.S. Wang, P.I. Hu, I.C. Lui, Rapid recovery of dilute copper from a simulated Cu-SDS solution with low-cost steel wool cathode reactor, J. Hazard. Mater. 163 (2009) 544-549.

[56] C. Kim, Y.L. Hsieh, Wetting and absorbency of nonionic surfactant solutions on cotton fabrics, Colloids Surf., A 187 (2001) 385-397.

[57] A. Helenius, D.R. McCaslin, E. Fries,C. Tanford, [63] Properties of detergents. Methods enzymol. 56 (1979) 734-749. 


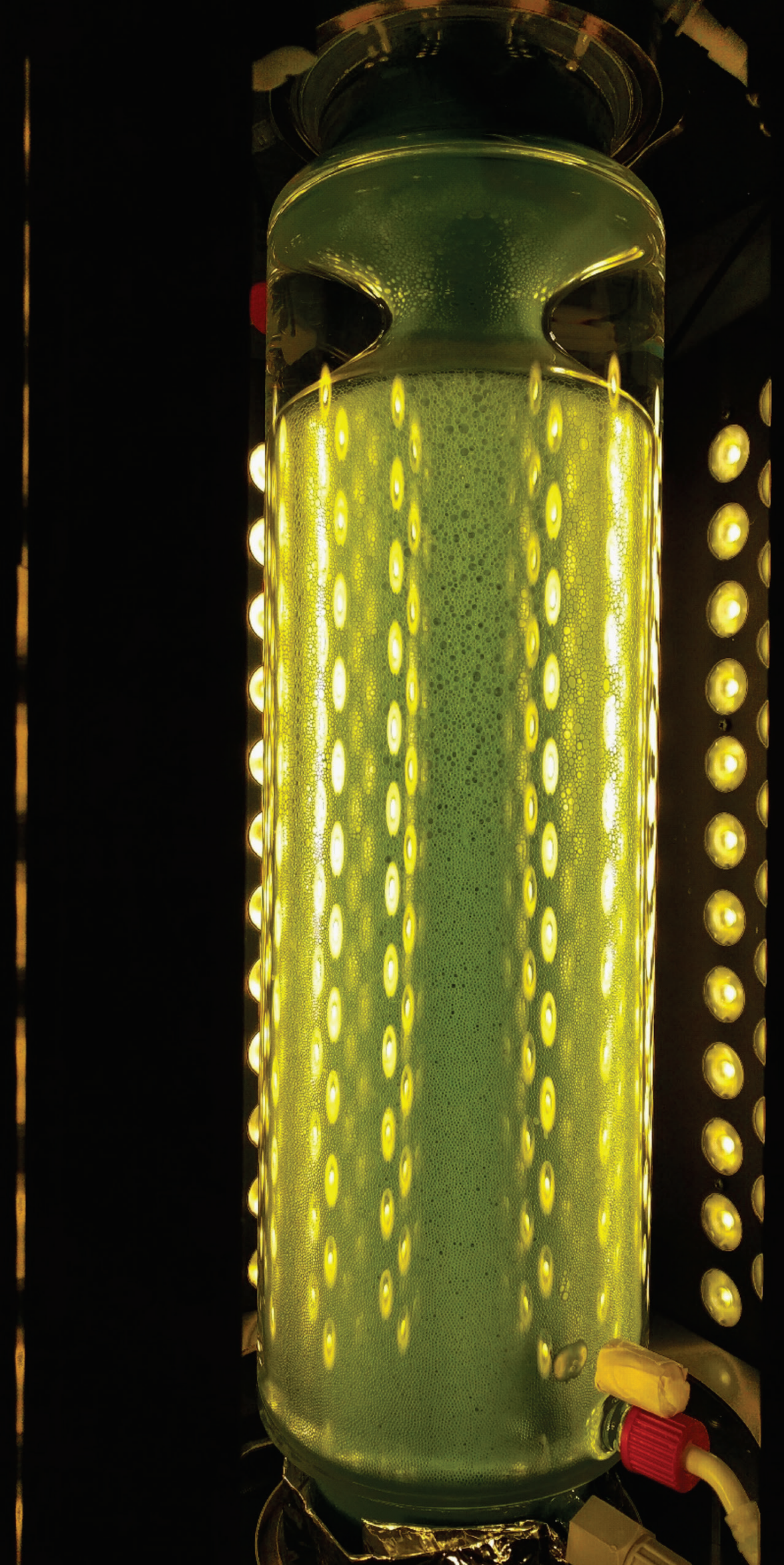




\title{
Chapter 4
}

\section{Improved liquid foam-bed photobioreactor design for}

\author{
microalgae cultivation
}

This chapter is submitted for publication as:
A. Janoska, R. Barten, S. de Nooy, P. van Rijssel, R. H. Wijffels, Marcel Janssen, Improved liquid foam-bed photobioreactor for microalgae cultivation. 


\begin{abstract}
The liquid foam-bed photobioreactor is a recently developed photobioreactor concept that allows for cost reduction in microalgae cultivation. Long term operation was not yet achieved, due to degradation of the surfactant. In this study, Pluronic F68 was applied for foam stabilization. In order to compensate for the low algae partitioning to Pluronic F68 stabilized foam, liquid recirculation was introduced into the reactor design. The microalgal suspension was continuously pumped from the bottom of the reactor to the top of the foam column where it was allowed to drain down again through the foam. This new design allowed increased mixing and, thereby, a homogenous algae distribution within the reactor. The volumetric mass transfer coefficient for $\mathrm{CO}_{2}$ in the foam-bed was $0.14 \mathrm{~s}^{-1}$, revealing that the gas transfer rate is an order of magnitude higher compared to bubble column reactors. This characteristic, together with a very high gas residence time, allows for a dramatic reduction in gas flow rate and a high carbon dioxide utilization efficiency. Long-term cultivation ( $>500 \mathrm{~h}$ ) of Chlorella sp. was achieved in a stable foam-bed. The areal productivity of the foam-bed photobioreactor was $57 \mathrm{~g} \mathrm{~m}^{-2} \mathrm{~d}^{-1}$, which is slightly lower than maximally achieved in flat panels under similar conditions. This is possibly related to substantial light scattering taking place in the foam leading to a steeper light gradient and increased reflection. The reactor dilution rate and the liquid recirculation rate were not optimized during our experiments and there is room for further improvement. During continuous reactor operation, biomass densities of more than $20 \mathrm{~g} \mathrm{~L}^{-1}$ could be maintained. This biomass density is a factor of 10 higher compared to traditional, liquid phase photobioreactors, thereby, contributing to reduced energy requirements for microalgae harvesting.
\end{abstract}




\subsection{Introduction}

Current photobioreactors are not yet economically feasible for mass production of microalgae [1-3]. In order to reduce production costs, an alternative photobioreactor design, utilizing foam instead of liquid for microalgae production was developed [4]. This liquid foam-bed photobioreactor is a promising microalgae cultivation concept, since it will allow for the reduction of harvesting costs due to the high biomass densities achieved. In addition, reduction in energy requirements are expected because of reduced gassing rates, enhanced mass transfer, and lower pressure drop in the foam-bed photobioreactor. The reduced gassing rates are achieved as a result of the enhanced mass transfer and increased gas residence time.

In the first explorative experiments on the foam-bed photobioreactor, the protein Bovine Serum Albumin was applied as surfactant. However, due to the loss of the foaming properties of this molecule, only short-term growth experiments could be carried out with a duration of 8 hours [4]. A detailed comparison of surfactants showed that Pluronic F68 is a promising surfactant for the foam-bed photobioreactor [5]. Pluronics are amphiphilic, non-ionic block copolymers, consisting of poly(ethylene oxide) and poly(propylene oxide) blocks [6]. Pluronics are widely used in cosmetics [7] or biomedical applications [8], and Pluronic F68 specifically is applied for shear protection in animal cell cultures [9]. When the liquid foam-bed photobioreactor is operated with Pluronic F68 as a surfactant instead of proteins, a longer operation time is expected since it showed no biodegradability in two week tests. In contrast to its good foaming properties and lack of toxicity, the drawback of this surfactant is the low microalgae partitioning to the foam phase. Therefore, the microalgal cells are not equally distributed over the foam and the underlying liquid phase; upon foaming the microalgal concentration is enhanced in the underlying liquid and reduced in the foam phase. Thus, a large fraction of the algal culture is not illuminated, resulting in reduced growth rates.

In order to eliminate the problem of low algal partitioning to the foam phase when Pluronic F68 is applied, a new reactor design was established that circumvents algal gradients in the reactor. The high density microalgal suspension underneath the foam-bed is pumped to the top of the reactor and allowed to drain back down through the foam-bed. This liquid recirculation ensures equal algae distribution in the reactor, and also provides better mixing in the foam phase. The significant mixing expected in the foam column underneath the liquid addition point is related to convective motion and internal circulation in the froth $[10,11]$. By distributing the microalgal 
broth on the top of the foam column, the additional liquid will drain down in the foam structure, thereby, enhancing the liquid fraction of the foams [12]. At higher liquid fractions, both coarsening and coalescence is reduced, therefore, also more stable foams are produced $[13,14]$. In foams without liquid addition, most of the liquid resides in the nodes and Plateau borders, while the liquid films are relatively thin (130-160 $\mu \mathrm{m}$ [15]). When considering mass transfer in foams, the thin films are prone to saturation $[16,17]$. With increasing liquid fraction the film thickness increases, which results in increased concentration gradient in the film and also film saturation is eliminated [18]. Together with better liquid advection [16], the additional liquid supply leads to increased mass transfer rates. Besides influencing mass transfer, the enhanced liquid fraction and algae concentration also alters the light profile in foams [19].

The formed foam has to be broken in order to refresh the gas phase in the bubbles entrapped, and ensure sufficient carbon dioxide $\left(\mathrm{CO}_{2}\right)$ for microalgal growth. The liquid jets originating from the liquid recirculation can serve as a foam breaking method [15, 20]. More specifically, foam breakage originates from the impact of the liquid droplets falling on the foam [21]. Spraying cell culture on foam was already reported to efficiently break foam [20]. Since the necessity of liquid recirculation also offers the possibility to break the foam, the need for external foam breakers is avoided.

This study aims to reveal the potential of a novel liquid foam-bed photobioreactor design with internal circulation of the microalgae suspension over the foam-bed. Although liquid recirculation might be beneficial for a range of non-ionic surfactants, this study is focused on the application of Pluronic F68 due to its good foaming characteristics and its low toxicity and biodegradability [5]. Batch and continuous operation were carried out in order to test the system and determine reactor productivity and photosynthetic efficiency. Additionally, the mass transfer coefficient, mixing characteristics, and the light profile was characterized in this new photobioreactor design.

\subsection{Materials and methods}

\subsubsection{The setup}

The foam-bed photobioreactor consists of a glass column surrounded by circular lights. The column is $55 \mathrm{~cm}$ high, has a diameter of $10 \mathrm{~cm}$ from height 0 to $6 \mathrm{~cm}$ and 49 to $55 \mathrm{~cm}$, and a diameter of $10.4 \mathrm{~cm}$ in between. The gas distributor plate is installed at $3 \mathrm{~cm}$ height from the bottom of the glass column. Between 44 and $50 \mathrm{~cm}$ height, the reactor has a narrowing; at 47 
$\mathrm{cm}$ height the column reaches the narrowest point with a diameter of $4 \mathrm{~cm}$. A water jacket used for temperature control surrounds the glass column. Foam was formed by a mixture of $\mathrm{N}_{2}$ and $\mathrm{CO}_{2}$ gas, supplied via mass flow controllers (Brooks instruments, The Netherlands). The gas entered the reactor via a distributor plate containing conical holes of $100 \mu \mathrm{m}$ diameter at the bottom and $30 \mu \mathrm{m}$ at the top, all holes were placed $3 \mathrm{~mm}$ from each other. The $\mathrm{N}_{2}$ flow was humidified through a water bottle at $2{ }^{\circ} \mathrm{C}$. After mixing the $\mathrm{N}_{2}$ and $\mathrm{CO}_{2}$ streams the gas dew point was analysed (E+E Elektronik dew point sensor). Finally, the gas was led over a filter (Whatman ${ }^{\circledR}$ PolyVENT 500, $0.2 \mu \mathrm{m}$ ) to sterilize the gas before entering to the reactor.

The algae suspension was recirculated from the bottom of the reactor to the top. The point of intake of the algae suspension was $2 \mathrm{~mm}$ above the gas distributer plate. It was pumped to the top of the foam column by two peristaltic pumps. Both streams were further divided into two more streams. The total liquid volume in the tubes of the recirculation system was $20 \mathrm{~mL}$. The resulting four streams were dispensed at the top of the reactor narrowing ( $5.5 \mathrm{~cm}$ below the lid) by four metal pipes with an inner diameter of $2 \mathrm{~mm}$. The falling liquid contributed to foam breaking. However, when complete foam deconstruction did not take place at a sufficient rate, the foam level was still maintained at $5.4 \mathrm{~cm}$ below the top by a level sensor, controlling the inflow of the gas. More specifically, when the foam was in contact with the level sensor the gas inflow was automatically and temporarily stopped until the foam-bed dropped again below the level sensor. The gas was forced to leave the reactor through a condenser at $2{ }^{\circ} \mathrm{C}$ after which the gas dew point was measured and gas composition analysed (infrared analyser for $\mathrm{CO}_{2}$ and a paramagnetic analyser for $\mathrm{O}_{2}$, Servomex, UK).

The temperature of the foam-bed was continuously monitored. A temperature probe was fitted inside a hollow metal tube filled with water, hanging in the foam bed $10 \mathrm{~cm}$ underneath the lid. Temperature regulation took place by an external cooling/heating unit recirculating water through the water jacket. The water temperature of this unit was adjusted manually to reach the desired temperature in the foam-bed. A pH probe was connected to a T-junction placed within the liquid recirculation line. In addition, a liquid sampling port was placed in the recirculation line. The foam-bed inside the cylindrical reactor was illuminated from all sides resulting in a homogenous light level over the reactor surface. More specifically, a circular light source was constructed, consisting of 8 vertical light panels placed around the column as an octagon. Each panel was composed of 24 warm-white LEDs (Avago ASMT-MY22-NMP00) equipped with a plastic lens with FHWM of $25.5^{\circ}$ (Part no. 10393, Carclo-optics, UK). 
The growth experiments were conducted in batch and chemostat modes. During chemostat mode media was pumped in and out by two peristaltic pumps. The inflow media bottle was continuously mixed with a magnetic stirrer to keep the surfactant dispersed and prevent its accumulation at the liquid surface. This media was sterilized by pumping through a $0.20 \mu \mathrm{m}$ filter (Sartolab P-20 or Sartobran 300, Sartorius, Germany). Reactor weight, weight of the media bottle (inflow), and weight of the harvest bottle (outflow) were measured by analytic balances. These balances were linked to a data acquisition system interfaced via a computer by means of a LabView (National Instruments, USA) virtual instrument allowing for continuous data logging and process control. The reactor weight and, as such, liquid volume were maintained constant by continuously adjusting the inflow pump rate, while the outflow pump was running at a fixed speed. A picture and a schematic overview of the setup is presented in Figure 4.1.
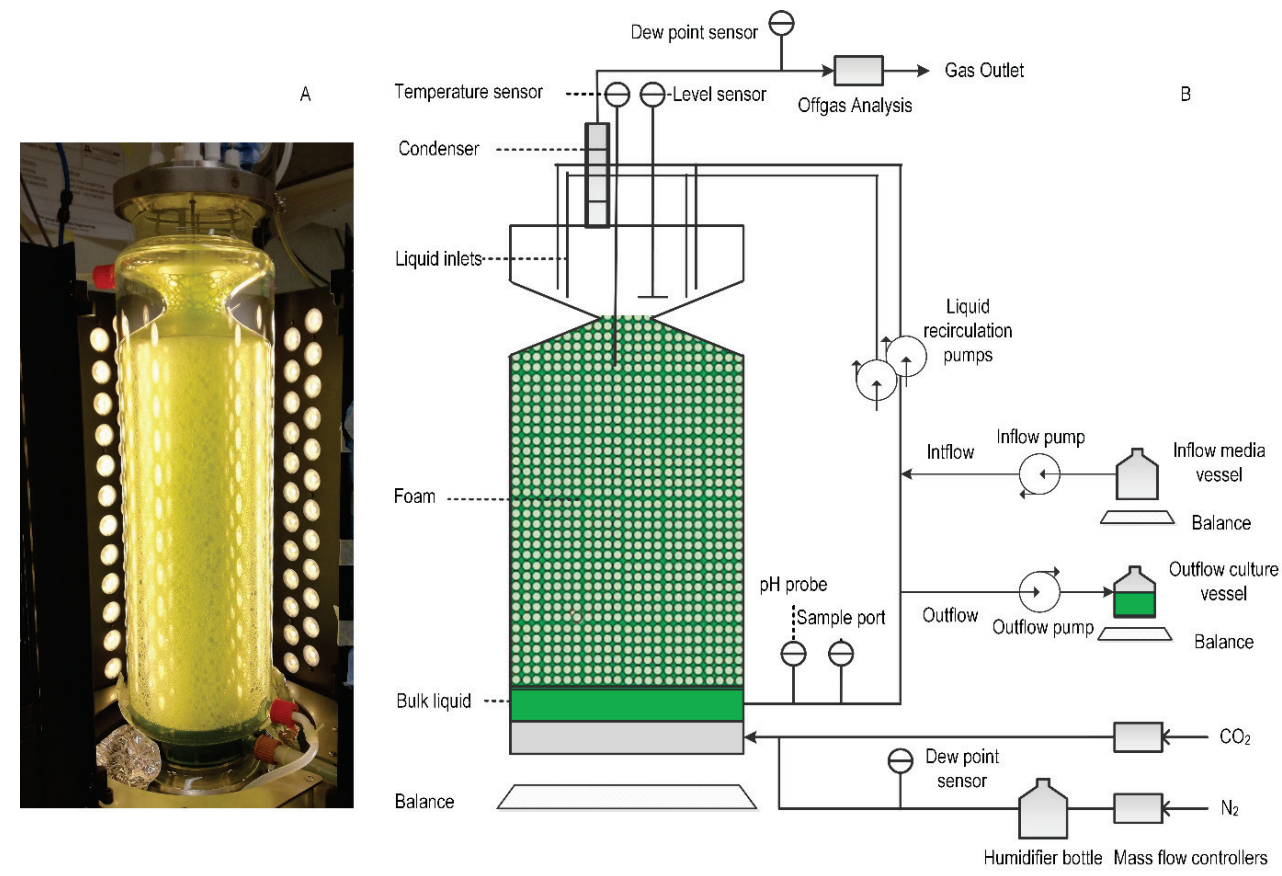

Figure 4.1. A) Picture of the foam-bed photobioreactor B) Schematic overview of the setup. 


\subsubsection{Reactor operation}

To monitor microalgal growth in the reactor, two samples were taken daily and analysed for optical density, cell count and biovolume (cell volume per liquid volume), PSII quantum yield, and microscopic analysis. Microalgal dry weight (DW) concentration was also measured daily from the outflow bottle during continuous operation with C. vulgaris. After the measurements, the outflow bottle was emptied, and the measured DW represents an average over 24 hours. For C. sorokiniana, the biovolume was translated to a dry weight value with a conversion factor of 0.5 gram dry weight per millilitre cell volume [22]. For C. vulgaris, following the direct DW measurements from the outflow bottle, a calibration curve was made and a correlation factor of 0.516 gram per millilitre was found, which was applied to convert biovolume to DW in the reactor samples.

The reactor was operated aseptically and was autoclaved only between operation with different strains. The volume at the bottom of the reactor directly above the sparger $(3 \mathrm{~cm})$ was covered with aluminium foil to prevent any algae from growing in the suspension underneath the foambed. Microalgal biofilms were re-suspended after each sampling moment using a magnet coated in silica gel. Biofilm formation depended on species and process conditions as will be discussed later. The Pluronic F68 concentration is expressed in terms of its critical micelle concentration (CMC) values. The CMC value of Pluronic F68 (Panreac, Applichem, A1288, BioChemica), was $0.334 \mathrm{~g} \mathrm{~L}^{-1}$.

A superficial gas flow speed of $0.61 \mathrm{~mm} \mathrm{~s}^{-1}$ was supplied into to the reactor, containing $16.1 \%$ $\mathrm{v} / \mathrm{v} \mathrm{CO}_{2}$ in $\mathrm{N}_{2}$. This gas flow was temporarily stopped in case foam breaking was not complete and the foam level reached the level sensor. Although the off-gas composition was continuously monitored, the analysis was not representative when the gas supply was stopped due to high foam levels. Therefore, in case of low measured $\mathrm{CO}_{2}$ concentrations in the off-gas, $\mathrm{CO}_{2}$ limitation was prevented by increasing the inlet $\mathrm{CO}_{2}$ concentration to $32.3 \%$. This way, the offgas $\mathrm{CO}_{2}$ concentration was increased to be above $5 \%$.

For C. sorokiniana, a total liquid volume of $470 \mathrm{ml}$ was used, however, for continuous operation, only $450 \mathrm{ml}$ was left due to sampling losses. During algae growth experiments the surfactant Pluronic F68 was added at a concentration of 0.5 CMC shortly before inoculation, thus, a foam-bed with only media was generated prior to inoculation. The temperature of the foam was maintained at $37^{\circ} \mathrm{C}$. During the first day of operation lower light intensities were applied to prevent photoinhibition after which the incident light intensity was increased to 1446 
$\mu \mathrm{mol} \mathrm{m} \mathrm{m}^{-2} \mathrm{~s}^{-1}$. The microalgal suspension was recirculated over the foam-bed at a superficial velocity of $0.59 \mathrm{~mm} \mathrm{~s}^{-1}$. For continuous reactor operation a dilution rate of $0.1 \mathrm{~h}^{-1}$ was applied.

For C. vulgaris, a continuous run was performed, at two different operational conditions. The reactor dilution rate was 0.064 and $0.049 \mathrm{~h}^{-1}$ and the superficial liquid recirculation speed was 0.38 and $0.73 \mathrm{~mm} \cdot \mathrm{s}^{-1}$, respectively. The liquid flow rate for continuous operation and the dilution rate was determined based on the weight increase of the outflow bottle, indicated by the balance underneath. The batch phase lasted for 46 hours after which continuous operation was initiated. The settings for continuous operation were changed again after 289 hours. A Pluronic F68 concentration of $0.4 \mathrm{CMC}$ was used. The reactor temperature was set at $35{ }^{\circ} \mathrm{C}$. Before inoculation, a foam-bed was formed with only surfactant and media. After inoculation the total liquid volume was $220 \mathrm{ml}$, and it was increased to $228.5 \mathrm{~mL}$ when the operational conditions were changed halfway the continuous run. Because of observed fluctuations in $\mathrm{pH}$, the $\mathrm{pH}$ was occasionally manually adjusted by $\mathrm{NaOH}$ addition. During the 2-days batch phase the light intensity was increased in multiple steps because of the possibility that $C$. vulgaris was more light sensitive than $C$. sorokiniana. During continuous operation an incident light level of $1205 \mu \mathrm{mol} \mathrm{m} \mathrm{m}^{-2} \mathrm{~s}^{-1}$ was maintained for C. vulgaris.

\subsubsection{Microalgae, inoculum cultivation conditions and media}

Chlorella sorokiniana (CCAP 221/8K) was obtained from the Culture Collection of Algae and Protozoa in Oban, Scotland. Chlorella vulgaris (MACC 4) was obtained from the Mosonmagyaróvár Algal Culture Collection in Hungary. However, 18S sequencing of this strain showed $99.9 \%$ similarity to C. sorokiniana, while only $99.6 \%$ similarity to C. vulgaris. The obtained DNA sequence is presented in Appendix 4.A. Both strains were cultivated using a 3 times concentrated M8a medium, as described by [4], with $60 \mathrm{mM}$ urea and $10 \mathrm{mM}$ phosphate salts. The $\mathrm{pH}$ of the medium was adjusted to 6.7 for $C$. sorokiniana and 7.4 for $C$. vulgaris. The media were filter sterilised using a Sartobran $3000.2 \mu \mathrm{m}$ filter (Sartorius, Germany).

Algal cultures were maintained in $250 \mathrm{ml}$ shake flasks with $100 \mathrm{ml}$ culture volume in an incubator operating at $25^{\circ} \mathrm{C}$, with orbital shaker set at $120 \mathrm{rpm}$. The light intensity was $35-40$ $\mu \mathrm{mol} \mathrm{m} \mathrm{m}^{-2}$ applied in a 16:8 day/night cycle at atmospheric $\mathrm{CO}_{2}$ concentration. Prior to reactor inoculation, daughter cultures were placed in another incubator with a $\mathrm{CO}_{2}$ concentration of $5 \%$ $\mathrm{v} / \mathrm{v}$ and stirring at $100 \mathrm{rpm}$ with a magnetic rod. In this homemade incubator the Erlenmeyer flasks were illuminated from below by a warm-white LED (BXRAW1200, Bridgelux, USA, 
Livermore). C. sorokiniana cultures were grown at a temperature of $37^{\circ} \mathrm{C}$ and with continuous light of $620 \mu \mathrm{mol} \mathrm{m} \mathrm{m}^{-2} \mathrm{~s}^{-1}$. C. vulgaris cultures were grown at $35{ }^{\circ} \mathrm{C}$ and $500 \mu \mathrm{mol} \mathrm{m} \mathrm{m}^{-2} \mathrm{~s}^{-1}$ continuous light supply.

The growth experiments and the mass transfer coefficient $\left(k_{L} a\right)$ and bubble size measurements were carried out with a different batch of surfactants (batch II, obtained Dec. 2016) compared to the experiments for liquid fraction, light distribution and mixing properties (batch I, obtained Oct. 2015). The produced foam was similar in terms of bubble size and foam stability at 0.5 CMC of batch II as batch I at 5 CMC.

\subsubsection{Analytical methods}

The optical density was measured in duplicate by a spectrophotometer (Hach-Lange DR6000) at 680 and $750 \mathrm{~nm}$.

The cell number and cell diameter were determined in duplicate with a Beckman Coulter Multisizer 3 (Beckman Coulter Inc.), using a $50 \mu \mathrm{m}$ aperture tube. Samples were diluted in 20 ml Isoton II diluent.

Dry weight measurements were carried out by vacuum-filtration of microalgal solution through a Whatman GF/F glass microfiber filters (diameter of $55 \mathrm{~mm}$, pore size $0.7 \mu \mathrm{m}$ ). Prior to filtration, the empty filters were pre-wet with de-mineralized water. After filtering the sample containing 1-10 $\mathrm{mg}$ algal biomass, the filter was rinsed twice with de-mineralized water. Before, and after the filtration, the filters were dried at $100{ }^{\circ} \mathrm{C}$ for 24 hours and, subsequently, cooled down in a desiccator for 2 hours before determining their weight.

The PSII quantum yield was measured using an Aquapen fluorimeter (AquaPen AP 100, PSI, Czech republic). The PSII quantum yield $\left(F_{v} / F_{m}\right)$ is a measure of the photosystem II efficiency [4]. Prior to measurements at $620 \mathrm{~nm}$ (C. sorokiniana) or $450 \mathrm{~nm}$ (C. vulgaris), the samples were placed for 15 minutes in dark.

Quantum yield $=\frac{F_{v}}{F_{m}}=\frac{F_{m}-F_{0}}{F_{m}}$

Equation 4.1

In Eq. 4.1, $F_{0}$ is the minimum fluorescence measured after dark adaptation and $F_{m}$ is the maximum fluorescence measured after applying the saturating light flash at $3000 \mathrm{umol} \mathrm{m} \mathrm{m}^{-2}$ [23]. 
The growth rate $\left(\mu, \mathrm{h}^{-1}\right)$, volumetric and areal productivity $\left(P_{V}, \mathrm{~g} \mathrm{~L}^{-1} \mathrm{~h}^{-1}\right.$ and $P_{A}, \mathrm{~g} \mathrm{~m}^{-2} \mathrm{~h}^{-1}$, respectively), and biomass yield on light $\left(Y_{x / p h}, \mathrm{~g}_{\mathrm{Cx}}\right.$ mol $\left._{\text {photons }}{ }^{-1}\right)$ during batch and continuous operation were calculated according to Eq. 4.2-4.5.

$$
\begin{aligned}
& \mu^{\text {batch }}=\frac{\ln \frac{C_{\chi}\left(t_{1}\right)}{C_{X}\left(t_{0}\right)}}{t_{1}-t_{0}} \quad \quad \mu^{\text {continuous }}=D=\frac{F_{L}}{V_{L}} \\
& P_{V}^{\text {batch }}=\frac{C_{x}\left(t_{1}\right)-C_{x}\left(t_{0}\right)}{t_{1}-t_{0}} \quad P_{V}^{\text {continuous }}=C_{x} \cdot \frac{F_{L}}{V_{L}} \\
& P_{A}^{\text {batch }}=\frac{P_{V} \cdot V_{L}}{A_{\text {illuminated }}} \quad P_{A}{ }^{\text {continuous }}=\frac{c_{X} \cdot F_{L}}{A_{\text {illuminated }}} \quad \text { Equation } 4.4 \\
& Y_{x / p h}=\frac{P_{A}}{E \cdot\left(3.6 \cdot 10^{-3}\right)}
\end{aligned}
$$

Where $C_{x}$ is the biomass density $\left(\mathrm{g} \mathrm{L}^{-1}\right), t$ is the time $(\mathrm{h}), V_{L}$ is the liquid volume $(\mathrm{L}), A_{\text {illuminated }}$ is the illuminated surface area of the reactor $\left(\mathrm{m}^{2}\right), E$ is the irradiating light intensity $\left(\mu \mathrm{mol} \mathrm{m} \mathrm{m}^{-2}\right.$ $\left.\mathrm{s}^{-1}\right), D$ is the dilution rate $\left(\mathrm{h}^{-1}\right)$ and $F_{L}$ is the liquid flow rate $\left(\mathrm{L} \mathrm{h}^{-1}\right)$. For batch cultivation, the linear growth phase was considered, while during continuous operation, the averaged steady state values were applied. The photons leaving the reactor as a result of transmission or reflection were neglected. For the calculations, the DW converted from cell volume measurements was applied for C. sorokiniana, while the measured DW from the outflow bottle was used in case of C. vulgaris.

\subsubsection{Retention time and mixing properties}

Mixing in the reactor was characterized by monitoring the flow of coloured liquid in the foam column. According to preliminary measurements, the retention time of biomass and ink did not differ significantly. Thus, instead of liquid recirculation, liquid containing ink (Parker Quink blue ink diluted 10x with demineralised water) was supplied at the top of the foam column via the liquid recirculation inlets, at 4 points in a circle of $4 \mathrm{~cm}$ diameter. The time required for the blue colour to arrive at the liquid layer underneath the foam-bed at the bottom of the reactor was monitored, and this time will be referred as the retention time. To ensure that ink drainage takes place homogenously in the foam column, pictures were taken every 10 seconds. The ink was supplied at a superficial velocity of $0.85 \mathrm{~mm} \mathrm{~s}^{-1}$. Foams were created by $\mathrm{N}_{2}$ gas, with a superficial velocity of $1.7 \mathrm{~mm} \mathrm{~s}^{-1}$, and the initial solution contained 5 CMC Pluronic F68 in a total of $400 \mathrm{~mL}$ solution. The effect of surfactant concentration, gas flow rates and ink addition rates were investigated. During the experiments with reduced gas flow rates, the foam was still formed with $1.7 \mathrm{~mm} \mathrm{~s}^{-1}$, and the gas flow rate was only changed to the desired one after the foam was formed. Measurements were conducted 3-5 minutes after the foam had formed in 
order to stabilise the foam at a certain condition and reach a steady liquid fraction. The level sensor based gas control was active during the stabilization time. During the measurement, the control was stopped.

\subsubsection{Liquid fraction and bubble size}

The liquid fraction and the bubble size of the foam was measured in order to calculate the specific surface area present in the foam-bed photobioreactor. The liquid fraction of the foam was determined at $5 \mathrm{CMC}$ surfactant concentration (batch I), $0.59 \mathrm{~mm} \mathrm{~s}^{-1}$ liquid recirculation superficial velocity and $0.79 \mathrm{~mm} \mathrm{~s}^{-1}$ superficial gas velocity. The liquid fraction was determined by quantifying the amount of liquid added or removed from the reactor to maintain $100 \mathrm{ml}$ liquid on the bottom of the reactor. It was assumed that all the liquid present in the reactor minus the $100 \mathrm{ml}$ at the bottom was in the foam phase.

The bubble size in the foam was determined after 30 minutes of the foam formation. A photographic image of the foam was taken at $9 \mathrm{~cm}$ and $35 \mathrm{~cm}$ above the gas distributer plate. Thirty five bubbles were measured with the help of a ruler that was included in the pictures. The ruler was placed outside the reactor, against the glass wall and the camera was aligned in the horizontal plane. For these measurements, a surfactant concentration of $0.5 \mathrm{CMC}$ was applied (batch II). The measurement was conducted at $0.48 \mathrm{~mm} \mathrm{~s}^{-1}$ superficial liquid recirculation velocity and $0.4 \mathrm{~mm} \mathrm{~s}^{-1}$ superficial gas velocity.

\subsubsection{Light distribution}

The fluence rate distribution in the reactor was measured inside the foam structure, at different positions with a $4 \pi$ spherical PAR quantum sensor (US_SQS/A, Walz Gmbh, Germany). Fluence rate was measured in duplicates at each point, where duplicates were created by measurement at the opposing sides of the reactor. Measurements were carried out in an empty reactor (cooling jacket filled with water), in a clear foam formed by 5 CMC Pluronic F68 (batch I), and with the same foam containing C. sorokiniana biomass (OD at $750 \mathrm{~nm}$ of 5.68, corresponding to $1.58 \mathrm{~g} \mathrm{~L}^{-1}$ biomass DW). Foams were formed with $\mathrm{N}_{2}$ gas at a superficial velocity of $1.7 \mathrm{~mm} \mathrm{~s}^{-1}$, but during the light measurements the gas flow was stopped. A liquid recirculation rate of $0.52 \mathrm{~mm} \mathrm{~s}^{-1}$ was applied. Measurements were done at 26 and $31 \mathrm{~cm}$ height from the gas distributor when the horizontal light attenuation was investigated. The spherical light sensor was positioned in the centre of the reactor by attaching it to a cord with a weight. Spacers were placed between the cord and the sensor for the light measurements at different 
positions in relation to the light exposed surface. In order to relate fluence rates to the irradiance, additional measurements were done with the flat $2 \pi$ PAR quantum sensor (SA-190, LiCor, USA) directly behind the glass wall in an empty reactor. A calibration curve for the irradiance as a function of lamp power was set up measured at 14, 26 and $36 \mathrm{~cm}$ height, and each height at 8 point, at three different light settings.

\subsubsection{Volumetric mass transfer coefficient determination}

The mass transfer coefficient $\left(k_{L} a\right)$ in the foam-bed photobioreactor was experimentally determined by measuring $\mathrm{O}_{2}$ transfer in the foam-bed. A method based on gas-phase analysis was applied [24] since it does not require measurement or sampling of the liquid phase, which is difficult when working with foams. In order to maximize the driving force for the transfer of $\mathrm{O}_{2}$ from gas to liquid, an oxygen scavenging compound, sodium sulphite $\left(\mathrm{Na}_{2} \mathrm{SO}_{3}, 50 \mathrm{~g} \mathrm{~L}^{-1}\right)$, was added to the liquid phase. As such, the dissolved oxygen concentration could be kept at 0 . In order to catalyse $\mathrm{O}_{2}$ scavenging by sulphite, cobalt chloride $\left(\mathrm{CoCl}_{2}\right)$ was added at $10 \mu \mathrm{mol}$ $\mathrm{L}^{-1}$ [25]. A temperature of $37^{\circ} \mathrm{C}$ was maintained in the reactor. A nitrogen-oxygen mixture was supplied for foam formation. In the first experiment, a superficial gas velocity of $0.79 \mathrm{~mm} \mathrm{~s}^{-1}$ was applied containing $13.61 \% \mathrm{v} / \mathrm{v}$ oxygen. In the second experiment, a superficial gas velocity of $0.59 \mathrm{~mm} \mathrm{~s}^{-1}$ was applied containing $15.87 \% \mathrm{v} / \mathrm{v}$ oxygen. The superficial liquid recirculation velocity during both experiments was $0.59 \mathrm{~mm} \mathrm{~s}^{-1}$. The foaming solution contained Pluronic F68 surfactant at a concentration of 5 CMC (batch II). The high surfactant concentration of 5 CMC was required since the solution applied $\left(50 \mathrm{~g} \mathrm{~L}^{-1} \mathrm{Na}_{2} \mathrm{SO}_{3}\right.$ and $\mathrm{CoCl}_{2}$ dissolved in demineralized water) resulted in decreased foam stability.

The $k_{L} a$ was also determined for the situation when the reactor was operated as a bubble column reactor. The reactor was filled up until the narrowing with demineralized water containing $\mathrm{Na}_{2} \mathrm{SO}_{3}$ and $\mathrm{CoCl}_{2}$, without surfactant. Gas was supplied at a superficial velocity of $0.59 \mathrm{~mm}$ $\mathrm{s}^{-1}$ containing $15.87 \% \mathrm{v} / \mathrm{v}$ oxygen. The reactor system was flushed with nitrogen prior to experiments.

The oxygen concentration of the ingoing and outgoing gas was monitored by off gas analysis. The outgoing gas was analysed after passing through a condenser at $2{ }^{\circ} \mathrm{C}$. The level sensor based control of the gas supply was not activated during the $k_{L} a$ measurements because a steady gas flow was required. In order to prevent the possibility of foam entering the off-gas analysis equipment a bottle with antifoam and a stirring magnet was introduced after the condenser. When a steady state was reached in the outgoing oxygen concentration, the obtained average 
steady state oxygen concentration was used to calculate the volumetric mass transfer coefficient $\left(k_{L} a\right)$. The $k_{L} a, \mathrm{~s}^{-1}$ was calculated according to Eq. 4.6 based on the molar fractions of $\mathrm{O}_{2}$ in the inflow and outflow gases ( $y_{\text {in }}$ and $y_{\text {out }}$, respectively), the constant molar flow rate of non-oxygen carrying gas $\left(G^{\text {carry }}\right.$, mol s$\left.{ }^{-1}\right)$, the foam-bed volume $\left(V_{F}, \mathrm{~m}^{3}\right)$, total pressure $\left(p_{T}\right.$, atm) and the Henry coefficient $\left(\mathrm{HO}_{\mathrm{O}}, \mathrm{m}^{3} \mathrm{~atm} \mathrm{~mol}^{-1}\right)$. This equation is based on a mass balance over the gas phase, and results in an average $k_{L} a$ value over the whole reactor.

$k_{L} a=-\frac{H_{O 2} \cdot G^{\text {carry }}}{p_{T} \cdot V_{F}}\left(\operatorname{Ln}\left(\frac{\frac{y^{\text {out }}}{1-y^{\text {out }}}}{\frac{y^{\text {in }}}{1-y^{\text {in }}}}\right)+\frac{y^{\text {out }}}{1-y^{\text {out }}}-\frac{y^{\text {in }}}{1-y^{\text {in }}}\right)$

Equation 4.6

$K_{L} a$ for $\mathrm{CO}_{2}$ was calculated using the square root of the ratio of the diffusion coefficients for $\mathrm{CO}_{2}$ and $\mathrm{O}_{2}$ [26]. The diffusion coefficients at $37^{\circ} \mathrm{C}$ and 1 atmosphere were calculated for $\mathrm{CO}_{2}$ and for $\mathrm{O}_{2}$ according to [27] and [28], respectively.

\subsection{Results and discussion}

\subsubsection{Mixing in the reactor}

Mixing in the foam-bed photobioreactor was visualized by replacing the recirculation flow at the top of the reactor by coloured liquid. Pictures taken of the ink drainage in the foam column are presented in Figure 4.2. At the moment the ink arrives to the bottom of the reactor ( after $54 \mathrm{~s}$ ), almost the whole reactor is coloured blue, indicating that the recirculated liquid also spreads in the horizontal direction. In other words, liquid drainage takes place equally over the whole reactor cross section and not only in the centre. The foam volume immediately below the neck where liquid distribution takes place, turned blue after approximately 60 seconds, indicating that in this volume the liquid mixing is relatively low. Potentially, mixing can be further improved by a re-design of the curvature of the neck.

Notable mixing, both in the horizontal and the vertical direction, takes place in the top of the foam-bed because of the continuous liquid supply, as also observed in previous studies $[10,11]$. Internal circulations in the foam might occur due to the difference in the density between wet and dry foam, since immediately underneath the liquid addition point, more wet foam is created [11]. 


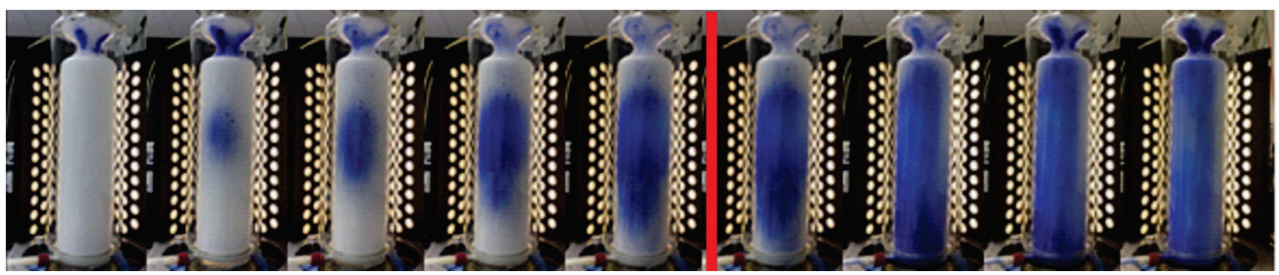

Figure 4.2. Distribution of blue ink over the foam-bed in time. Each picture was taken at a 10 second interval starting from the left. The moment when the ink arrives to the bottom of the reactor (retention time) is at $54 \mathrm{~s}$, which is between the $5^{\text {th }}$ and $6^{\text {th }}$ picture, indicated with a red vertical line.

The liquid retention time in the foam-bed was investigated in foams formed under different conditions. The retention time is defined as the time required for the ink to arrive at the bottom of the reactor. With increasing ink supply rate, mimicking increased superficial liquid recirculation velocity, the liquid retention time decreases (Figure 4.3A). This is related to an increase in the net downward superficial liquid velocity, which is due to both the increased superficial liquid addition rates and also the enhanced gravity induced drainage due to increased liquid fractions [12]. Since the net downwards superficial liquid velocity increases faster than the liquid fraction, the retention time will be reduced. At the lowest superficial liquid velocity, the liquid distribution was not equal and, therefore, the measurements do not represent homogeneous drainage over the whole foam column.

Due to the enhanced counter-current gas supply, the upward going liquid flux is elevated, and the liquid retention time increases at the highest gas flow rate tested (Figure 4.3B). The effect of gas flow rate on the liquid retention time, however, is small compared to the effect of the recirculating liquid flow. Also, increased surfactant concentration were found to result in an increased retention time. This is possibly related to a reduction of bubble size at increased surfactant concentrations, as described previously [4]. Smaller bubbles lead to a decrease of the liquid drainage rate [12], and, therefore, the supplied liquid resides longer in the foam structure. The retention time increases by $28 \%$ at a three times increased surfactant concentration of 15 CMC (data not shown). 

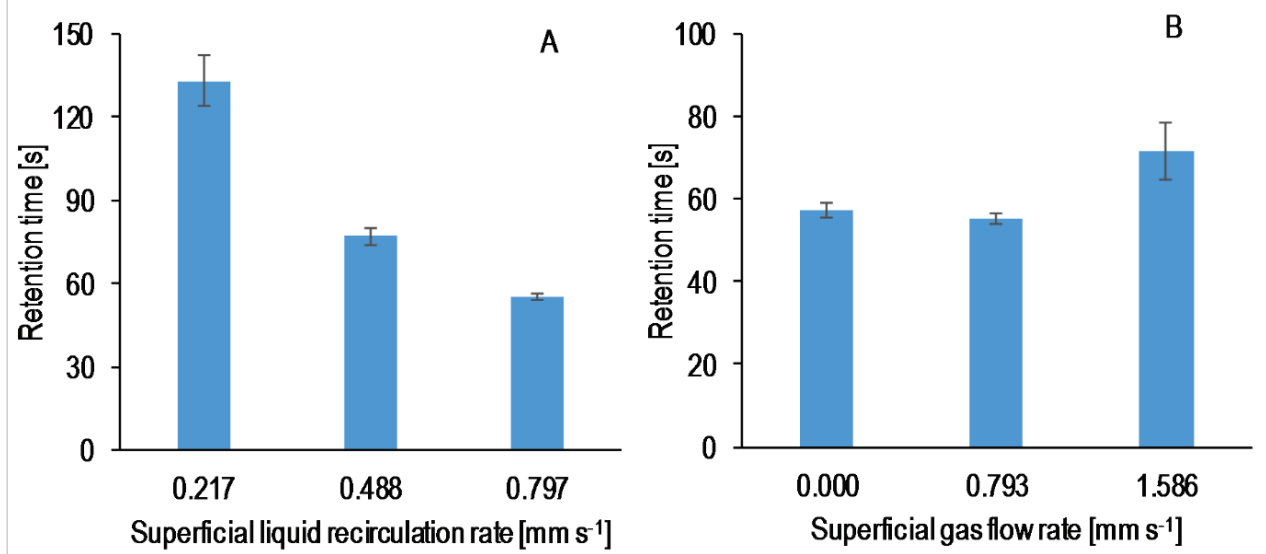

Figure 4.3. Retention time in the foams formed at A) different superficial liquid recirculation velocities; B) different superficial gas velocities. The error bars represent the standard deviation of triplicate measurements.

\subsubsection{Light distribution}

Since foam has a highly scattering nature due to multiple gas-liquid interfaces, which all reflect and refract light, diffuse light is generated inside the foam structure [19]. Therefore, photons are scattered towards multiple directions, and the photon flux microalgal cells perceive can be best described by the flux incident on a sphere of unit cross section, called fluence rate. Fluence rate is measured with a spherical light sensor, which collects light from all directions and is equally sensitive for each direction. In contrast, during the growth experiments, we refer to the photon flux density at the bioreactor light exposed surface, which is measured with a flat sensor collecting light only from a single hemisphere. This flat sensor is cosine corrected and is, thus, less sensitive for large angles of incidence.

The measurements illustrated in Figure 4.4A show that the fluence rate in the empty reactor is higher than the photon flux density (irradiance in Figure 4.4A) at the reactor surface. Since the reactor is illuminated from all sides, a considerable difference between photon flux density and fluence rate can be observed. In a real liquid foam-bed the fluence rate is even higher than in the situation of an empty reactor (Figure 4.4A). This is related to the light scattering behaviour of the foam. Light rays will be scattered many times when passing through a foam-bed, resulting in a more diffuse (isotropic) light field. In clear foam, a peak can be observed at $1.4 \mathrm{~cm}$ depth, after which the fluence rate continuously decreases towards the centre of the reactor because of light scattering. The fluence rate peaking a given distance from the light exposed surface is related to a photon flux developing in the opposite direction as the incident light flux because 
of multiple scattering events in the foam. This backward flux also leads to substantial light loss form the reactor and it adds to the reflection of light at the reactor surface.

In the case where light absorbing microalgae are present within the foam, the fluence rate will quickly drop when moving deeper in the foam-bed, towards the direction of the central axis (Figure 4.4B). Already at a microalgae concentration of $1.58 \mathrm{~g} \mathrm{~L}^{-1}$, no light is present in the centre of the reactor at an irradiance of $1607 \mu \mathrm{mol} \mathrm{m} \mathrm{m}^{-2} \mathrm{~s}^{-1}$, indicating that at higher biomass concentration a significant dark volume is present in the centre of the foam column. The vertical fluence rate distribution in the foam column was not homogenous and these results are presented in Appendix 4.B.
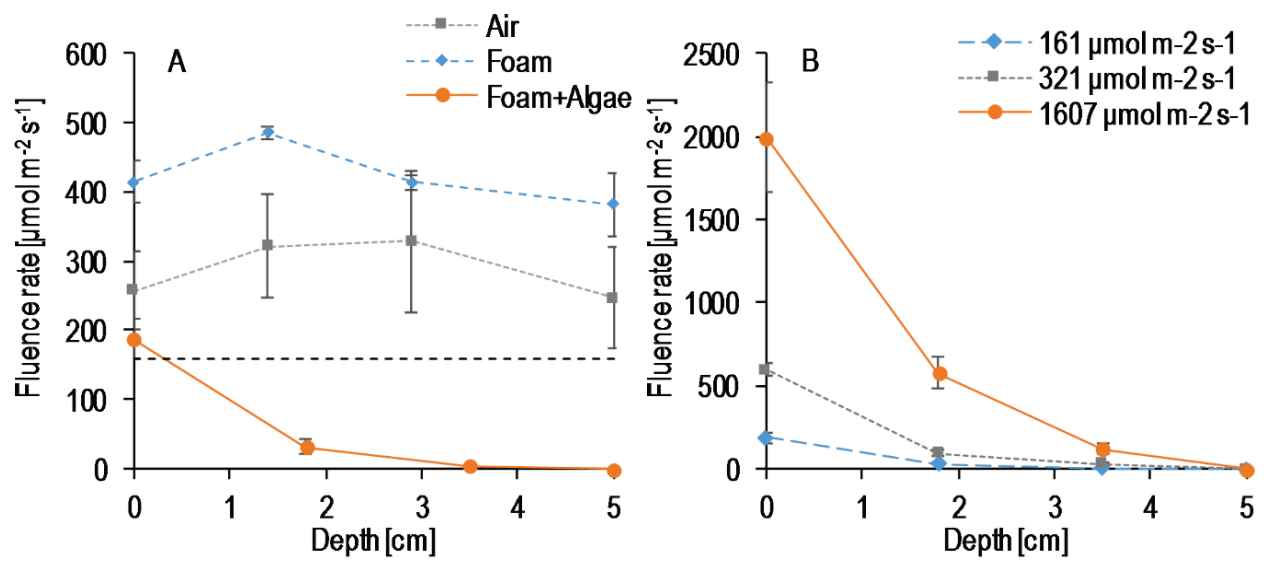

Figure 4.4. A) Horizontal light profile in the bioreactor filled with air, clear foam, and microalgae enriched foams at $160 \mu \mathrm{mol} \mathrm{m} \mathrm{m}^{-2} \mathrm{~s}^{-1}$ irradiance. B) Horizontal light attenuation in foams with different photon flux densities: 161, 321 and $1607 \mu \mathrm{mol} \mathrm{m} \mathrm{m}^{-2} \mathrm{~s}^{-1}$ at a biomass concentration of $1.6 \mathrm{~g} \mathrm{~L}^{-1}$.

\subsubsection{Determination of the mass transfer coefficient}

The mass transfer coefficient of the lab-scale foam-bed photobioreactor was experimentally determined. The first experiment at $0.79 \mathrm{~mm} \mathrm{~s}^{-1}$ superficial gas velocity lead to an average $k_{L} a$ of $0.141 \mathrm{~s}^{-1}$, while the second experiment at $0.59 \mathrm{~mm} \mathrm{~s}^{-1}$ superficial gas velocity resulted in a $k_{L} a$ of $0.113 \mathrm{~s}^{-1}$ (Table 4.1). The $k_{L} a$ for $\mathrm{CO}_{2}$ is $98 \%$ of the value for $\mathrm{O}_{2}$ when calculated with the square root of the ratio of the diffusion coefficients, resulting in a value of $0.135 \mathrm{~s}^{-1}$ and $0.108 \mathrm{~s}^{-1}$, respectively. The reduction of $k_{L} a$ at reduced superficial gas velocity is a well-known phenomenon [29, 30], and is most likely related to reduced mixing and turbulence [31]. The obtained $k_{L} a$ values are equal to, or slightly higher than, $k_{L} a$ values measured in other foam-bed systems $[16,32]$. 
In order to compare the $k_{L} a$ values of foam-bed photobioreactors with bubble column reactors, the foam-bed photobioreactor was also operated as a bubble column reactor. Triplicate measurements resulted in an average $k_{L} a$ value for $\mathrm{O}_{2}$ of $0.019 \mathrm{~s}^{-1}$. This value is higher than reported for other bubble column photobioreactors of $0.001-0.007 \mathrm{~s}^{-1}$ [33], possibly due to the reduced bubble size in our reactor, which was equipped with a gas distributor with very small holes. Thus, under the same conditions the $k_{L} a$ for foam-bed operation was 6 fold higher than for bubble column operation.

Table 4.1. The average $\mathrm{O}_{2}$ concentration in the inlet and outlet gas, the superficial gas velocity applied and the calculated volumetric mass transfer coefficient, $\mathrm{k}\left\llcorner\right.$ a for both $\mathrm{O}_{2}$ and $\mathrm{O}_{2}$ gases in the foam-bed photobioreactor during operation as a foam-bed and as a bubble column.

\begin{tabular}{lccccc}
\hline & $\begin{array}{c}\mathrm{O}_{2} \text { in } \\
{[\% \mathrm{v} / \mathrm{v}]}\end{array}$ & $\begin{array}{c}\mathrm{O}_{2} \text { out } \\
{[\% \mathrm{v} / \mathrm{v}]}\end{array}$ & $\begin{array}{c}\text { Superficial gas } \\
\text { velocity }\left[\mathrm{mm} \mathrm{s}^{-1}\right]\end{array}$ & $\begin{array}{c}k_{\llcorner} \mathrm{O} \mathrm{O}_{2} \\
{\left[\mathrm{~s}^{-1}\right]}\end{array}$ & $\begin{array}{c}k_{\llcorner} \mathrm{a} \mathrm{CO} \\
{\left[\mathrm{s}^{-1}\right]}\end{array}$ \\
\hline Foam-bed experiment 1 & 13.61 & 1.47 & 0.79 & 0.141 & 0.135 \\
Foam-bed experiment 2 & 15.87 & 1.44 & 0.59 & 0.113 & 0.108 \\
Bubble column experiment & 15.87 & 11.22 & 0.59 & 0.019 & 0.019 \\
Literature value foam-bed [16] & - & - & $11-14.7$ & 0.011 & $0.12-0.14$ \\
\hline
\end{tabular}

The specific surface area in the foam-bed photobioreactor can be calculated when the bubble size and the liquid fraction are known. An average bubble size of $0.74 \mathrm{~mm}$ was measured at $0.48 \mathrm{~mm} \mathrm{~s}^{-1}$ superficial liquid recirculation velocity and $0.4 \mathrm{~mm} \mathrm{~s}^{-1}$ superficial gas velocity. The liquid fraction of the foam was $11.9 \%$ at $0.59 \mathrm{~mm} \mathrm{~s}^{-1}$ liquid recirculation superficial velocity and $0.79 \mathrm{~mm} \mathrm{~s}^{-1}$ superficial gas velocity. At increased liquid recirculation and gas flow rates, the liquid fraction of the foam increased and the bubble size slightly decreased. In order to calculate a rough estimate of the specific surface area, the differing experimental conditions for the bubble size and liquid fraction measurements were neglected. The specific surface area can be calculated according to [16], and results in a value of $7142 \mathrm{~m}^{-1}$, which is more than double reported for other foam-bed systems [16]. This can be explained by the small bubbles created in our reactor, however, the possibility of underestimation of bubble size cannot be excluded. Bubble size measurements by image analysis of pictures taken at the reactor wall result in an underestimation of real bubble size [34, 35].

According to our calculations, the specific surface area in a foam-bed is 10 to 140 fold higher than that in a bubble column reported by Perry et al. [16]. Since the increase of $k_{L} a$ is smaller than the increase of specific surface area in a foam-bed compared to a bubble column, the liquid 
side mass transfer coefficient $k_{L}$ in foam-beds must be lower than those in bubble column reactors. This reduction in $k_{L}$ is possibly related to the presence of surfactants [36, 37] attaching to the bubble surfaces, to small and less mobile bubbles [36, 38], or reduced superficial velocities [39] in the foam-bed photobioreactor. The stagnant boundary layer is possibly more rigid due to the presence of surfactants and reduced bubble sizes in the foam-bed, and also it is thicker to reduced slip velocity between the gas and the liquid phases.

During algal growth experiments, higher $k_{L} a$ values are expected because the ionic strength of the algal cultivation medium is lower than the ionic strength of the sodium sulphite solution used during the $k_{L} a$ determination. According to Jamnongwong et al., the ionic strength has an effect on the mass transfer by altering the diffusion coefficient [40]. Following the calculations of Solomon [41], the ionic strength introduced by $\mathrm{Na}_{2} \mathrm{SO}_{3}$ is $1.19 \mathrm{~mol} \mathrm{~L}^{-1}$, which corresponds to a greater than $27 \%$ reduction of the diffusion coefficient for $\mathrm{O}_{2}$ [40]. Thus, the $k_{L} a$ value during microalgal growth in the liquid foam-bed photobioreactor probably is higher than the value determined in this experiment.

\subsubsection{Chlorella sorokiniana batch growth in the foam-bed photobioreactor}

Two experiments were carried out in order to evaluate specific growth rate, reactor productivity, and maximal cell density in a foam-bed photobioreactor operated in batch mode. The measured biovolume concentrations were converted to dry weight concentration and the resulting growth curve is presented in Figure 4.5. The maximal specific growth rate achieved was $0.23 \mathrm{~h}^{-1}$ assuming exponential growth during the first 3 hours. This growth rate approaches the maximum specific growth rate of $0.27 \mathrm{~h}^{-1}$ [42]. In the following linear growth phase, light limitation occurred. The more rapid increase in biomass concentration during run 2 is related to an earlier increase of light level. During the stationary phase (47.5-70 hours), algal growth was possibly limited by nutrients instead of light. The average biomass concentration during the stationary phase was $21.7-25.7 \mathrm{~g} \mathrm{~L}^{-1}$. The maximal possible cell density of $C$. sorokiniana when grown on the medium was determined at $22.26 \mathrm{~g} \mathrm{~L}^{-1}$ based on the nitrogen concentration in the medium, the cell composition and C-molar weight reported by Duboc et.al. [43]. During the stationary phase, the dry weight still increased slightly, while the cell number (not shown) and photosynthetic efficiency decreased. This can be explained by accumulation of storage compounds due to nitrogen starvation $[44,45]$. The photosynthetic efficiency was measured as the quantum yield of PSII photochemistry (see Appendix 4.C). The quantum yield decreased after 40 hours, which is an indication of nitrogen limitation [46]. 
During the experiment the rate of foam breaking was lower than the rate of foam formation and, therefore, the gas supply was frequently stopped when foam reached the foam level sensor. The time that gas was supplied into the reactor was found to be $25 \%$ of the total time in which microalgae were cultivated (Table 4.2 and Appendix 4.D). This translates into an average superficial gas velocity of $0.16 \mathrm{~mm} \mathrm{~s}^{-1}$. The dew point measurements demonstrated that the amount of water vapour leaving the system was negligible: less than $1 \mathrm{~mL}$ water has evaporated during the whole run. The $\mathrm{pH}$ was not actively regulated. The average $\mathrm{pH}$ values during the two runs were 6.5 and 6.7, varying between $\mathrm{pH}$ values of 6.1 and 7.6 (see Table 4.2 for averages and Appendix 4.D for more details). Variations are partly caused by the change in dissolved $\mathrm{CO}_{2}$ concentration resulting from varying microalgal $\mathrm{CO}_{2}$ uptake during the batch run. For another part $\mathrm{pH}$ variations are related to the discontinuous gas supply. Since fluctuating $\mathrm{pH}$ results in sub-optimal growth conditions for C. sorokiniana, higher growth rates could have been obtained if the $\mathrm{pH}$ was regulated [47]. The temperature of the reactor was $36^{\circ} \mathrm{C}$ on average (Table 4.2 and Appendix 4.D). The small difference between the optimal growth temperature of $37{ }^{\circ} \mathrm{C}$ and the actual temperature was due to the indirect temperature control by manual adjustment of the temperature of the water bath. Foam stability during the batch runs declined, as observed by bigger bubbles and reduced liquid fraction. In order to maintain the original foam stability, occasional surfactant addition was necessary.

During the batch experiments with $C$. sorokiniana, biofilms from the reactor surface were removed twice a day. In the time periods in between, however, a thin biofilm developed repeatedly. Additional batch experiments without biofilm removal (data not shown) demonstrated that algae growth was severely reduced due to biofilm formation as we observed a $50 \%$ reduction in growth rate and maximal biomass density. 


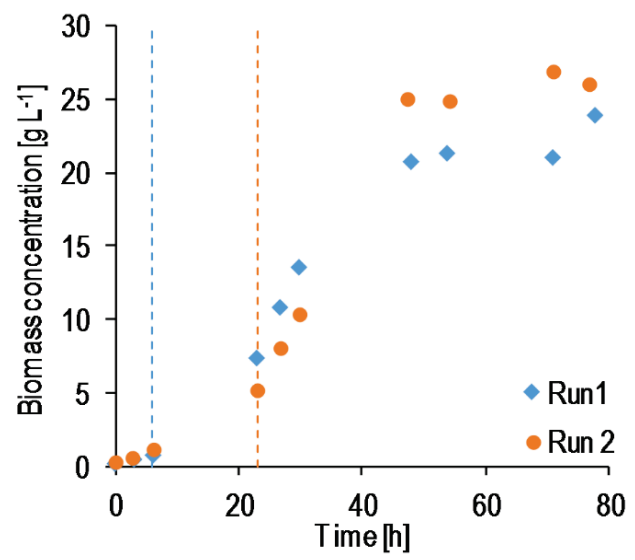

Figure 4.5. Growth curve of $C$. sorokiniana obtained in two batch cultivation experiments. Cultivation conditions: superficial gas velocity $0.61 \mathrm{~mm} \mathrm{~s}^{-1} ; 470 \mathrm{~mL}$ of liquid culture with $0.5 \mathrm{CMC}$ Pluronic F68; superficial liquid recirculation rate $0.59 \mathrm{~mm} \mathrm{~s}^{-1}$. Light intensity was increased from 482 to $1446 \mu \mathrm{mol} \mathrm{m}^{-2} \mathrm{~s}^{-1}$ after $6 \mathrm{~h}$ (run 1) and 23 h (run 2).

Table 4.2. Average $\mathrm{pH}$, temperature (T), relative time period of gas supply and PSIl quantum yield during all microalgae cultivation experiments in the liquid foam-bed photobioreactor. For $\mathrm{pH}$ and temperature the actual range of variation is indicated by minimal and maximal values observed. The detailed measurements are presented in Appendix 4.D. For the batch experiments, the averages of the whole operation are presented, while for continuous operation only the averages during the steady-states (circled boxes in Figure 4.6 and 4.7) are shown.

\begin{tabular}{|c|c|c|c|c|c|c|c|c|}
\hline & & & $\begin{array}{c}\text { Average } \\
\mathrm{pH}\end{array}$ & $\begin{array}{l}\text { Min- } \\
\max \mathrm{pH}\end{array}$ & $\begin{array}{c}\text { Average } \\
\mathrm{T}\left[{ }^{\circ} \mathrm{C}\right]\end{array}$ & $\begin{array}{c}\text { Min-Max T } \\
{\left[{ }^{\circ} \mathrm{C}\right]}\end{array}$ & $\begin{array}{c}\text { Period } \\
\text { gassing } \\
{[\%]}\end{array}$ & $\begin{array}{l}\text { PSII Quantum } \\
\text { yield }\end{array}$ \\
\hline \multirow[t]{2}{*}{ Batch } & \multirow[t]{2}{*}{ C. sorokiniana } & 1 & 6.70 & $6.3-7.6$ & 36.0 & $33.8-36.4$ & 25.7 & $\begin{array}{l}<40 \mathrm{~h}: 0.71 \\
>40 \mathrm{~h}: 0.54-0.66\end{array}$ \\
\hline & & 2 & 6.46 & $6.1-7.0$ & 36.1 & $34.7-37.4$ & 25.1 & $\begin{array}{l}<40 \mathrm{~h}: 0.72 \\
>40 \mathrm{~h}: 0.55-0.70\end{array}$ \\
\hline \multirow{4}{*}{ Chemostat } & \multirow{2}{*}{ C. sorokiniana } & 1 & 7.01 & $6.5-7.6$ & 35.5 & $35.2-36.1$ & 16.1 & 0.68 \\
\hline & & 2 & 6.81 & $6.6-7.2$ & 35.9 & $35.6-36.3$ & 21.4 & 0.70 \\
\hline & \multirow{2}{*}{ C. vulgaris } & 1 & 6.55 & $6.5-6.7$ & 32.4 & $31.4-34.0$ & 100.0 & 0.71 \\
\hline & & 2 & 6.56 & $6.2-8.1$ & 31.4 & $29.8-34.0$ & 73.8 & 0.70 \\
\hline
\end{tabular}

\subsubsection{Continuous cultivation of $C$. sorokiniana in the foam-bed photobioreactor}

Continuous cultivation of $C$. sorokiniana was performed in the foam-bed photobioreactor. First microalgae were grown under a batch regime for 20 hours, after which the chemostat mode was initiated. At the end of the batch phase, an average cell density of $3.6 \mathrm{~g} \mathrm{~L}^{-1}$ was reached and a dilution rate of $0.1 \mathrm{~h}^{-1}$ was introduced. The evolution in biomass concentration during the 
experiment is shown in Figure 4.6. The biomass concentrations within the red square were assumed to be representative of a steady state situation (run 1: 70.2 - 167.2 hours, and run 2: 64.6-143.3 hours). The growth pattern of the duplicate experiments were similar, and the steady state biomass concentrations were 6.1 and $7.5 \mathrm{~g} \mathrm{~L}^{-1}$ on average. The steady state lasted only 34 days, since after this time period biomass concentration started to decline due to profound biofilm formation.

The average PSII quantum yield was 0.68 and 0.70 during the steady states of the two duplicate runs. This quantum yield was identical to that of the inoculum, indicating that the efficiency of photosystem II of the C. sorokiniana cells was not affected by the cultivation conditions, such as the presence of surfactant, or the shear stresses introduced by foam formation and liquid recirculation [4]. While the average $\mathrm{pH}$ was close to the optimum $\mathrm{pH}$ of $6.7, \mathrm{pH}$ values of 7.6 were observed during the first run, which might reason the slightly reduced quantum yield during run 1 compared to run 2 (Table 4.2).

Gas supply to the reactor was active less than $25 \%$ of the time because of the foam level frequently reaching the level sensor. This indicates that the foam stability was too high and/or the foam breaking by the liquid recirculation supplied on the top of the foam column was not efficient. Based on visual observation of bubble size and liquid fraction the foam stability in this experiment was increased compared to the batch operation. This is possibly related to the continuous surfactant supply of fresh surfactant via the media inflow. Surfactant concentration analysis indeed demonstrated that at the end of the batch phase, the surfactant concentration in the liquid phase was less than half compared to the average value during continuous chemostat operation (see Appendix 4.E). The severe decrease in surfactant concentration during a batch can be explained by degradation or absorption of the molecule. The average surfactant concentration in the liquid phase during continuous operation was $30 \%$ of the surfactant concentration in the inlet media, which is presumably related to surfactant molecules accumulating in the foam phase and also to their degradation or adsorption.

During steady state, samples taken from the bioreactor showed an oscillating pattern in the dry weight concentration. This repetitive difference between consecutive samples is possibly related to biofilm formation. Algal biofilms shade light from the cells present in the foam, resulting in a reduction in the measured steady-state biomass concentrations. After cleaning the walls, the removed biofilm temporarily increase the liquid phase concentration. Biofilm formation was quicker and more severe at the end of the experiments, possibly caused by the 
selection for biofilm formation due to the chemostat operation. An additional measurement of the dry weight concentration in the outflow bottle at the end of the experiment (Run 2) resulted in a 1.35 times higher biomass concentration in comparison to the reactor sample. The biomass concentration in the outflow bottle presumably is higher because it was collected over a 24 hour period and, thus, included part of the biomass originating from the biofilm removal. The reactor samples were always taken prior to biofilm removal and, therefore, the biomass concentrations presented in Figure 4.6 do not represent the real capability of the system. Calculating bioreactor productivity from these reactor samples will lead to an underestimation of the productivity including biofilm formation.

The significance of biofilm formation was further demonstrated by additional experiments without biofilm removal (data not shown). In those experiments, the maximal biomass concentration reached was only $5.3 \mathrm{~g} \mathrm{~L}^{-1}$, and steady state could not be reached. Because of the increasing biofilm thickness the light supply to the liquid phase continuously decreased, resulting in biomass wash-out.

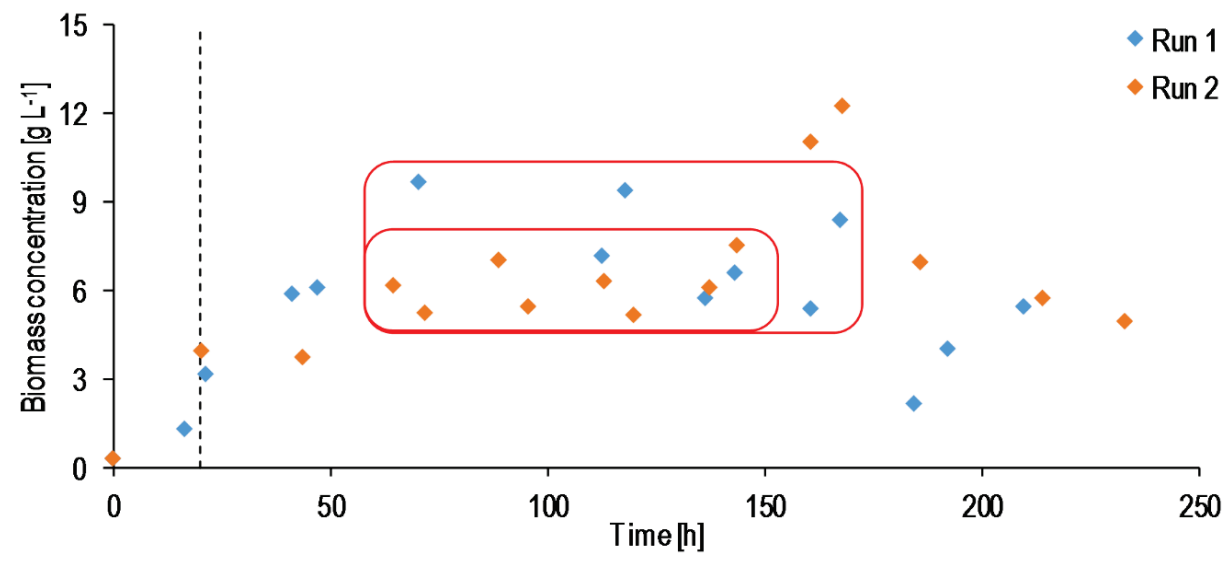

Figure 4.6. Microalgal dry weight concentration in the foam-bed photobioreactor during two identical chemostat experiments. The measured microalgal biovolume concentration was converted to dry weight by means of a calibration factor value. The dashed vertical line indicates the point in time where reactor dilution was started. Operational conditions: dilution rate $0.1 \mathrm{~h}^{-1}$; superficial gas velocity $0.61 \mathrm{~mm} \mathrm{~s}^{-1} ; 450 \mathrm{~mL}$ of liquid culture with 0.5 CMC Pluronic F68; superficial liquid recirculation rate $0.59 \mathrm{~mm} \mathrm{~s}^{-1}$. Light intensity was increased from 482 to 1446 $\mu \mathrm{mol} \mathrm{m} \mathrm{m}^{-2} \mathrm{~s}^{-1}$ after $20 \mathrm{~h}$

\subsubsection{Continuous cultivation of $C$. vulgaris}

In order to achieve a longer operational time and also a longer steady state period, an algal strain was required that has reduced ability for biofilm formation compared to C. sorokiniana. 
Chlorella vulgaris MACC4, a strain closely related to C. sorokiniana, showed less biofilm formation in other studies in our laboratory. This strain was, therefore, tested in the foam-bed photobioreactor. In order to accommodate for residual biofilm formation the daily procedure of bringing the biofilm back into suspension by scraping the bioreactor surface was maintained, but liquid samples were also taken from the outflow bottle. Sampling the outflow bottle, instead of sampling the reactor, holds the advantage that the removed biofilms are contained within. Moreover, since the outflow was collected over 24 hours, dry weight measurements in Figure 4.7 represent the complete $24 \mathrm{~h}$ interval before the given time point.

The effect of two different operational conditions on the performance of the foam-bed photobioreactor was investigated. The operational conditions were differing in terms of dilution rate and liquid recirculation rate. The recirculation rate was lowered to $61 \%$ of the recirculation rate during the reactor runs with $C$. sorokiniana, $0.38 \mathrm{~mm} \mathrm{~s}^{-1}$, in order to investigate the possibility of more energy-efficient operation. For this modification, the diameter of the inlet tubes had to be reduced to $1 \mathrm{~mm}$ in order to ensure equal liquid flows through the 4 tubes. In addition, this resulted in continuous liquid jets, which appeared to be more effective to break foam than discontinuous dripping. The liquid volume in the reactor was reduced compared to the previous experiments in order to minimize the liquid layer underneath the foam-bed, and to accommodate for the reduced liquid fraction in the foam at reduced recirculation rate.

The batch growth period lasted for 46 hours, where a specific growth rate of $0.175 \mathrm{~h}^{-1}$ was reached in the first 3 hours of operation, at a photon flux density of $241 \mu \mathrm{mol} \mathrm{m} \mathrm{m}^{-2} \mathrm{~s}^{-1}$. At the end of the batch phase, a biomass concentration of $25.9 \mathrm{~g} \mathrm{~L}^{-1}$ was achieved according to microalgal biovolume determinations from the liquid phase (Figure 4.7). After this batch phase the chemostat mode was initiated with a dilution rate of $0.064 \mathrm{~h}^{-1}$. The light intensity during continuous operation was $1205 \mu \mathrm{mol} \mathrm{m} \mathrm{m}^{-2} \mathrm{~s}^{-1}$. The biomass concentrations within the first red square in Figure 4.7 were used to define the steady state situation. The average biomass density in the outflow during steady state was $20.2 \mathrm{~g} \mathrm{~L}^{-1}$, while the reactor measurements indicated 22.4 $\mathrm{g} \mathrm{L} \mathrm{L}^{-1}$.

The low recirculation rate resulted in a dry foam and, therefore, the foam stability was reduced due to coarsening and coalescence being more dominant in dry foams, contributing to foam collapse $[13,14]$. The foam volume decreased, and the foam height was generally below the level sensor. Due to the dry foam, the level sensor based foam volume control did not work properly; dry foam has low conductivity and was, therefore, not detected. Consequently, 
continuous gas supply took place and occasionally foam overflow left the reactor through the gas outlet. This liquid loss led to an underestimation of the reactor dilution rate.

After the first steady state, at 289 hours, the recirculation rate was doubled to $0.73 \mathrm{~mm} \mathrm{~s}^{-1}$. A steady state biomass concentration of $21.6 \mathrm{~g} \mathrm{~L}^{-1}$ was achieved between 333.3-504.8 hours according to measurements from both the reactor and the outflow bottle. This is similar to the biomass concentration achieved during the previous steady state, possibly due to the simultaneous variation of two parameters. Not only the recirculation rate changed, but it appeared that also reactor dilution rate changed. More specifically, since higher recirculation rates increase the liquid fraction of the foam, the liquid layer underneath the foam column was minimal. Therefore, a mixture of foam and liquid filled up the recirculation tubing, and the outflow pump also transported some bubbles leading to a reduction in the dilution rate. The dilution rate was determined to be $0.049 \mathrm{~h}^{-1}$. The reduced dilution rate is expected to result in increased biomass densities, while the increased recirculation rate results in increased liquid fraction in the foam, which contributes to increased self-shading of algal cells.

Since foam stability increased because of a wetter foam at higher recirculation rate, the foam level control was active. On average gas was supplied to the reactor $73.8 \%$ of the time during steady state (Table 4.2 and Appendix 4.D). The increased gassing time compared to the $C$. sorokiniana reactor experiments are possibly due to reduced foam stability, which can be related to the application of a lower surfactant concentration of $0.4 \mathrm{CMC}$ instead of $0.5 \mathrm{CMC}$. Clear correlations could be observed between the $\mathrm{pH}$ fluctuations and the irregular gas supply. In case the gas supply was stopped, the $\mathrm{pH}$ values rose (data presented in Appendix 4.D) because the dissolved $\mathrm{CO}_{2}$ concentration dropped and bicarbonate dissociated to $\mathrm{CO}_{2}$ and $\mathrm{OH}^{-}$ . The $\mathrm{pH}$ varied between 6.2 and 8.1 , which might have caused a reduction in growth rate during the steady state (Table 4.2 and Appendix 4.D).

In conclusion, when $C$. vulgaris was cultivated, high biomass densities could be maintained in the reactor for prolonged time periods. This long operational time (21 days) and stable steady state biomass concentrations achieved, compared to C. sorokiniana, are explained by the severe reduction of biofilm formation when $C$. vulgaris was cultured. After 21 days the reactor operation was stopped, although foam stability was not compromised.

Steady state biomass concentrations of $C$. vulgaris under batch and continuous growth conditions are really close to the maximum biomass concentration the growth media could support. Since the PSII quantum yield showed small deviations during steady state and was on 
average 0.70 (Table 4.2 and Appendix 4.C), we conclude that no severe nutrient limitation occurred.

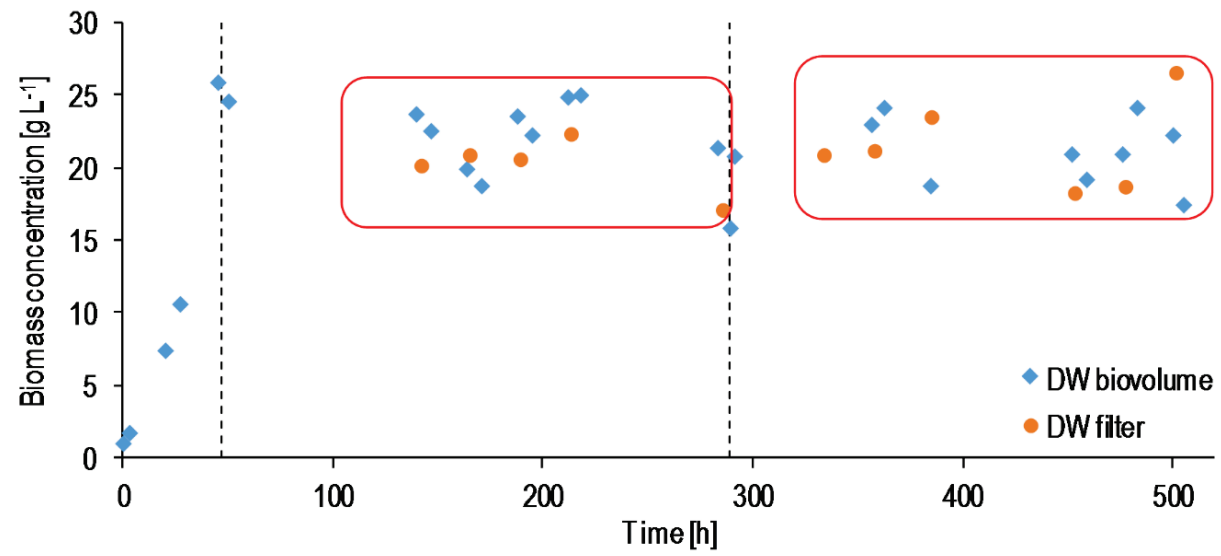

Figure 4.7. Microalgal dry weight concentration of $C$. vulgaris in a continuously diluted foam-bed photobioreactor. Biomass concentration was determined by biovolume measurements from the reactor (DW biovolume) and also by direct dry weight measurements from the outflow bottle (DW filter). Light intensity was changed from 241 to 482 , 804 and $1205 \mu \mathrm{mol} \mathrm{m}{ }^{-2} \mathrm{~s}^{-1}$ after 21, 27 and $45 \mathrm{~h}$. The left dashed vertical line indicates the point in time where reactor dilution was started. Operational conditions from 46 to $289 \mathrm{~h}$ : dilution rate $0.064 \mathrm{~h}^{-1}$; superficial liquid recirculation rate $0.38 \mathrm{~mm} \mathrm{~s}^{-1} ; 220 \mathrm{~mL}$ liquid culture with $0.4 \mathrm{CMC}$ Pluronic F68; superficial gas velocity $0.61 \mathrm{~mm} \mathrm{~s}$ 1. The second dashed line indicates a change of operation condition: dilution rate $0.049 \mathrm{~h}^{-1}$; superficial liquid recirculation rate $0.73 \mathrm{~mm} \mathrm{~s}^{-1} ; 228.5 \mathrm{~mL}$ liquid culture.

\subsubsection{Evaluation of the foam-bed photobioreactor performance}

Comparing the reactor performance to a previous liquid foam-bed photobioreactor design, significant improvements were achieved during batch operation. In that study, the same algal species but a different surfactant, BSA was applied [4]. The maximal reactor operation time reported in the previous study was only 8 hours. Longer reactor runs were not possible since the surfactant lost its foaming properties with time. The most relevant improvement, therefore, is the stability of the system, which allowed continuous cultivation for long time periods. The possibility of longer cultivations originates from the use of a non-degradable surfactant, Pluronic F68, which allowed for 3 weeks of continuous reactor operation and possibly more.

These stable and longer runs enabled the evaluation of the photobioreactor in terms of maximal biomass densities achieved, volumetric and areal reactor productivity and yield on light, as presented in Table 4.3. For the batch experiments, values were calculated for the light limited linear growth phase [48], while for chemostat experiments the steady state biomass concentration was used. 
In terms of areal productivities and yield on light the batch experiments performed slightly better than the chemostat cultivation experiments. For the batch experiments, the linear growth phase was considered, where the biomass densities were between 5-15 $\mathrm{g} \mathrm{L}^{-1}$. Although in batch cultivation biomass concentrations are continuously changing, the results indicate that the optimum biomass concentration for maximal productivity is within the above mentioned range. When the optimal biomass concentration is applied, chemostat experiments are expected to result in comparable productivities, since the culture is constantly kept in the linear growth phase. Although the biomass concentration of $C$. sorokiniana in continuous experiments were close to the ones of the batch run, the areal productivity and yield on light was lower than in the linear phase under batch conditions. This is possibly because the removed biofilms remained in the reactor during batch operation, which is in contrast to continuous runs where this additional biomass was partially pumped out without being measured. Additionally, during the longer chemostat experiments, the biofilm formation was more severe compared to the batch runs, possibly due to selection for biofilm-formation.

Comparing the chemostat experiments with $C$. sorokiniana and C. vulgaris, the runs with $C$. sorokiniana were shorter and the steady state was less stable due to more intense biofilm formation. This biofilm formation of $C$. sorokiniana cells must have negatively affected the measured productivity and yield on light because the biomass grown in algal biofilms were partly not measured and the biofilm will shade the cells growing in the foam. However, still slightly higher biomass productivity was reached during the $C$. sorokiniana runs, possibly due to the cell concentration being closer to the optimal biomass concentration for maximal productivity. From the tested dilution rates, the highest value, $0.1 \mathrm{~h}^{-1}$ resulted in the highest areal productivity, suggesting that biomass concentrations around 6-8 $\mathrm{g} \mathrm{L}^{-1}$ are optimal and further reduction in dilution rate does not result in increased productivities. The high biomass concentrations achieved during C. vulgaris cultivation show that long-term cultivation at high densities is feasible and foams are stable with 20-25 $\mathrm{g} \mathrm{L}^{-1}$ biomass. Although such a high concentration is suboptimal for this reactor, it would be practical in a thinner system.

When comparing our results to values from literature achieved in flat panel systems, yield on light and the areal productivity reached in the foam-bed photobioreactor are lower than in flat panels (Table 4.3). Suboptimal dilution rate, biomass concentration (C. vulgaris) and biofilm formation (C. sorokiniana) were already discussed before. Another important factor contributing to this lower productivity of the foam-bed is related to the light scattering nature of foam [49]. Scattering results in a steeper light gradient in the photobioreactor, since part of 
the photons change direction and will not take the shortest path towards the centre of the reactor. In fact a photon flux will develop in the opposing direction. Close to the light-exposed surface the fluence rate, therefore, is higher than the irradiance, leading to oversaturation of the microalgal photosystem, while the centre of the reactor has insufficient light supply. Additionally, surface reflection and bulk reflection originating from the scattered light orientated backwards lead to light losses [50] and, thereby, contributing to the reduced productivity.

In the studies by Tuantet et al. [51] and Cuaresma et al. [52] a range of dilution rates were tested and the optimal dilution rate for the maximal productivity is presented in Table 4.3. Since in our study no deliberate optimization was performed, higher productivities might be achieved when optimal dilution rates are applied. For example, in the study by Cuaresma et. al., at a dilution rate of $0.12 \mathrm{~h}^{-1}$, only $60 \%$ of the productivity expected was achieved [52]. Additionally, at high liquid fractions or high biomass concentrations, the yield on light is reduced as maintenance becomes a more dominant factor. In the interior of the reactor, and in the liquid layer underneath the foam-bed, where no light is available, the culture will convert internal biomass for maintenance purposes and, therefore, the biomass yield on light will decrease [53]. Results presented in Figure 4.4 already demonstrated that even at $1.6 \mathrm{~g} \mathrm{~L}^{-1}$ biomass concentration, no light is present in the centre of the reactor, implying that when the biomass concentration is increased to $20 \mathrm{~g} \mathrm{~L}^{-1}$, a substantial part of the reactor is dark. To summarize, increased dilution rates, thinner reactors and reduced liquid fractions will further increase productivities. Additionally, the productivity can be improved when the liquid volume in the reactor that is not illuminated is minimized. In these experiments, the liquid layer on the bottom of the reactor and in the tubing account for 20 to $50 \%$ of the total liquid volume.

Further improvements of the foam-bed photobioreactor must be realized aiming at more efficient foam breaking, resulting in a more stable foam-bed volume. This will also allow for continuous gas supply and, thus, also continuous $\mathrm{CO}_{2}$ supply rate. In our study, $\mathrm{CO}_{2}$ limitations were prevented by applying high $\mathrm{CO}_{2}$ concentrations. However, high $\mathrm{CO}_{2}$ uptake efficiency can only be achieved when the $\mathrm{CO}_{2}$ supply is continuous, which would consequently also prevent $\mathrm{pH}$ fluctuations. More efficient foam breaking can be achieved when re-designing the top of the foam column, and additionally by reducing the foam stability. This can be achieved by reduced surfactant concentrations or reduced gas and liquid flow rates. 
Despite the reduced yield on light and areal productivity results compared to traditional flat panel systems, the foam-bed photobioreactor holds great potential for obtaining high cell density microalgae biomass, which introduces a cost reduction for downstream processing [4]. The achieved high biomass densities can be further improved when reduced liquid recirculation rates or thinner reactors are applied. In a foam-bed, a minimum factor of 3.5 increase in biomass concentration can be achieved compared to flat panels at similar dilution rates and light intensities [52], indicating that the foam-bed photobioreactor is advantageous when aiming for high biomass densities.

Table 4.3. Summary of the reactor performance during batch and chemostat cultivation of $C$. vulgaris and $C$. sorokiniana in the foam-bed photobioreactor: biomass density $C_{x}$, areal productivity $r_{x}$ areal , biomass yield on light $Y_{x / p h}$, volumetric productivity $r_{x}^{\text {volumetric }}$ and dilution rates $D$. $C_{x}$ represents an average value during steady state for the chemostat operation, or the average biomass density during stationary phase in batch cultivations. Operational conditions C. sorokiniana: liquid recirculation rate $0.59 \mathrm{~mm} \mathrm{~s}^{-1}$; liquid volume $470 \mathrm{ml}$ (batch) or $450 \mathrm{ml}$ (chemostat), light intensity $1446 \mu \mathrm{mol} \mathrm{m}^{-2} \mathrm{~s}^{-1}$. Operational conditions $C$. vulgaris: liquid recirculation rate 0.38 and $0.73 \mathrm{~mm} \mathrm{~s}^{-1}$ (1 and 2 in table below); liquid volume 220 and $228.5 \mathrm{ml}$ ( 1 and 2 in table), light intensity $1205 \mu \mathrm{mol} \mathrm{m}^{-2} \mathrm{~s}^{-1}$. The flat panel experiments were done under the following conditions: 1) $2100 \mu \mathrm{mol} \mathrm{m} \mathrm{m}^{-2} \mathrm{~s}^{-1}, 14 \mathrm{~mm}$ light path 2) $1500 \mu \mathrm{mol}$ $\mathrm{m}^{-2} \mathrm{~s}^{-1}, 14 \mathrm{~mm}$ light path 3) $1530 \mu \mathrm{mol} \mathrm{m} \mathrm{m}^{-2} \mathrm{~s}^{-1}$, illuminated from both sides, $10 \mathrm{~mm}$ light path.

\begin{tabular}{|c|c|c|c|c|c|c|}
\hline $\begin{array}{l}\text { Operational } \\
\text { mode }\end{array}$ & Culture strain & Run & $\begin{array}{c}C_{x} \\
{\left[g^{-1}\right]}\end{array}$ & $\begin{array}{c}r_{x}^{\text {areal }} \\
{\left[g^{-2} \mathrm{~h}^{-1}\right]}\end{array}$ & $\begin{array}{c}Y_{x / p h} \\
{\left[\mathrm{~g} \mathrm{~mol}_{\mathrm{ph}^{-1}}\right]}\end{array}$ & $\begin{array}{c}D \\
{\left[\mathrm{~h}^{-1}\right]}\end{array}$ \\
\hline \multirow{2}{*}{$\begin{array}{c}\text { Batch } \\
\text { foam-bed }\end{array}$} & \multirow{2}{*}{ C. sorokiniana } & 1 & 21.72 & 2.92 & 0.57 & - \\
\hline & & 2 & 25.66 & 2.43 & 0.47 & - \\
\hline \multirow{4}{*}{$\begin{array}{l}\text { Chemostat } \\
\text { foam-bed }\end{array}$} & \multirow{2}{*}{ C. sorokiniana } & 1 & 7.49 & 2.36 & 0.45 & 0.1 \\
\hline & & 2 & 6.12 & 1.93 & 0.37 & 0.1 \\
\hline & \multirow{2}{*}{ C. vulgaris } & 1 & 20.23 & 1.98 & 0.46 & 0.064 \\
\hline & & 2 & 21.55 & 1.70 & 0.39 & 0.049 \\
\hline \multirow{3}{*}{ Flat Panel } & \multirow{3}{*}{ C. sorokiniana } & $1[52]$ & 2.1 & 7.7 & 1.0 & 0.24 \\
\hline & & $2[54]$ & 2.3 & 4.96 & 0.86 & turbidostat \\
\hline & & $3[51]$ & 11 & 5.3 & 0.98 & $0.1-0.15$ \\
\hline
\end{tabular}

\subsection{Conclusions}

A novel liquid foam-bed photobioreactor design was successfully employed for microalgae cultivation. Chlorella sorokiniana and Chlorella vulgaris were grown efficiently when Pluronic F68 was applied as a surfactant. In order to compensate for the low algae partitioning to the foam phase, liquid recirculation was introduced to the reactor design. This recirculation ensured 
homogenous algae distribution in the reactor. The new design together with the good foaming characteristics of Pluronic F68 enabled increased foam stability and long-term operation $(<500$ h). Mixing in the reactor was facilitated by the liquid recirculation, resulting in homogeneous distribution of the recirculated liquid in the axial direction. The retention time of the recirculated liquid in the foam phase was $54 \mathrm{~s}$. The mass transfer coefficient in the foam-bed $\left(k_{L} a\right)$ was an order of magnitude higher compared to a bubble column reactor operated under the same conditions. The increased rate of $\mathrm{CO}_{2}$ transfer together with a longer gas residence time will allow for reduced gas supply requirements of the foam-bed. For the first time, continuous cultivation of microalgae in a foam-bed photobioreactor was realised. An areal productivity of $2.4 \mathrm{~g} \mathrm{~m}^{-2} \mathrm{~h}^{-1}$ was achieved, which is expected to further improve when optimized dilution rates and liquid recirculation rates are applied. A further increase of productivity can be achieved when the foam breaking is improved and the liquid volume underneath the foam column is minimized. During continuous operation, a cell density above $20 \mathrm{~g} \mathrm{~L}^{-1}$ was maintained. Due to the increased gas transfer capacity, and the high biomass densities achieved, the liquid foambed photobioreactor presents a promising design that might contribute to reduced energy requirements. 


\section{Appendix 4.A: 18S sequence of Chlorella vulgaris MACC4}

GTATAAACTGCTTTATACTGTGAAACTGCGAATGGCTCATTAAATCAGTTATAGTTTATTTGATGGTACCTACT ACTCGGATACCCGTAGTAAATCTAGAGCTAATACGTGCGTAAATCCCGACTTCTGGAAGGGACGTATTTATTA GATAAAAGGCCGACCGGGCTCTGCCCGACTCGCGGTGAATCATGATAACTTCACGAATCGCATGGCCTTGC GCCGGCGATGTTTCATTCAAATTTCTGCCCTATCAACTTTCGATGGTAGGATAGAGGCCTACCATGGTGGTA ACGGGTGACGGAGGATTAGGGTTCGATTCCGGAGAGGGAGCCTGAGAAACGGCTACCACATCCAAGGAAG GCAGCAGGCGCGCAAATTACCCAATCCTGACACAGGGAGGTAGTGACAATAAATAACAATACTGGGCCTTTT CAGGTCTGGTAATTGGAATGAGTACAATCTAAACCCCTTAACGAGGATCAATTGGAGGGCAAGTCTGGTGCC AGCAGCCGCGGTAATTCCAGCTCCAATAGCGTATATTTAAGTTGCTGCAGTTAAAAAGCTCGTAGTTGGATTT CGGGTGGGGCCTGCCGGTCCGCCGTTTCGGTGTGCACTGGCAGGGCCCACCTTGTTGCCGGGGACGGGC TCCTGGGCTTCACTGTCCGGGACTCGGAGTCGGCGCTGTTACTTTGAGTAAATTAGAGTGTTCAAAGCAGGC CTACGCTCTGAATACATTAGCATGGAATAACACGATAGGACTCTGGCCTATCCTGTTGGTCTGTAGGACCGG AGTAATGATTAAGAGGGACAGTCGGGGGCATTCGTATTTCATTGTCAGAGGTGAAATTCTTGGATTTATGAAA GACGAACTACTGCGAAAGCAtTTGCCAAGGATGTTTTCATTAATCAAGAACGAAAGTTGGGGGCTCGAAGAC GATTAGATACCGTCCTAGTCTCAACCATAAACGATGCCGACTAGGGATCGGCGGATGTTTCTTCGATGACTC CGCCGGCACCTTATGAGAAATCAAAGTTTTTGGGTTCCGGGGGGAGTATGGTCGCAAGGCTGAAACTTAAA GGAATTGACGGAAGGGCACCACCAGGCGTGGAGCCTGCGGCTTAATTTGACTCAACACGGGAAAACTTACC AGGTCCAGACATAGTGAGGATTGACAGATTGAGAGCTCTTTCTTGATTCTATGGGTGGTGGTGCATGGCCGT TCTTAGTTGGTGGGTTGCCTTGTCAGGTTGATTCCGGTAACGAACGAGACCTCAGCCTGCTAAATAGTCACG GTTGGTTCGCCAGCCGGCGGACTTCTTAGAGGGACTATTGGCGACTAGCCAATGGAAGCATGAGGCAATAA CAGGTCTGTGATGCCCTTAGATGTTCTGGGCCGCACGCGCGCTACACTGATGCATTCAACGAGCCTAGCCT TGGCCGAGAGGCCCGGGTAATCTTTGAAACTGCATCGTGATGGGGATAGATTATTGCAATTATTAATCTTCAA CGAGGAATGCCTAGTAAGCGCAAGTCATCAGCTTGCGTTGATTACGTCCCTGCCCTTTGTACACACCGCCCG TCGCTCCTACCGATTGGGTGTGCTGGTGAAGTGTTCGGATTGGCGACCGGGGGCGG 


\section{Appendix 4.B: Vertical fluence rate distribution in a liquid foam- bed photobioreactor}

The vertical fluence rate distribution in the liquid foam-bed photobioreactor was not homogenous (Figure 4.B.1). In case the reactor contained clear foam, at approximately $26 \mathrm{~cm}$ height from the sparger, the fluence rate started steeply decreasing until the narrow part was reached. Immediately below the narrowing (measured at $41 \mathrm{~cm}$ height) the fluence rate decreased to approximately half of the value measured at lower positions at the central axis. This decrease is possibly due to the narrowing of the reactor where light escapes via the cooling chamber. The same phenomena was observed when the fluence rate was measured in air, but the decrease was more prominent.

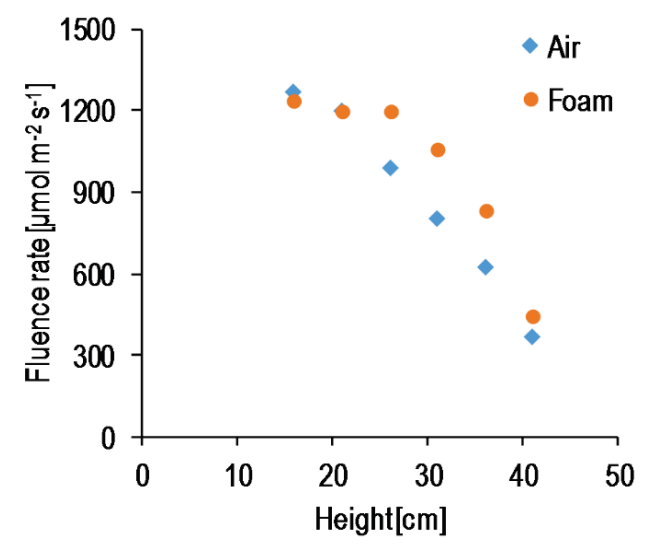

Figure 4.B.1. Fluence rate distribution in the liquid foam-bed photobioreactor as a function of reactor height at 321 $\mu \mathrm{mol} \mathrm{m}-2 \mathrm{~s}^{-1}$ irradiance. $0 \mathrm{~cm}$ height indicates where the sparger was placed, and the reactor narrowing was between 44 and $50 \mathrm{~cm}$, with the narrowest point at $47 \mathrm{~cm}$ height. 


\section{Appendix 4.C: Quantum yield of PSII photochemistry}

The PSII quantum yield of $C$. sorokiniana and $C$. vulgaris cells during cultivation in the liquid foam-bed photobioreactor are presented in Figure 4.C.1. The quantum yield during the batch cultivation of $C$. sorokiniana declined, possibly because of nutrient limitation. During the chemostat operation the PSII quantum yield of both strains remained constant.
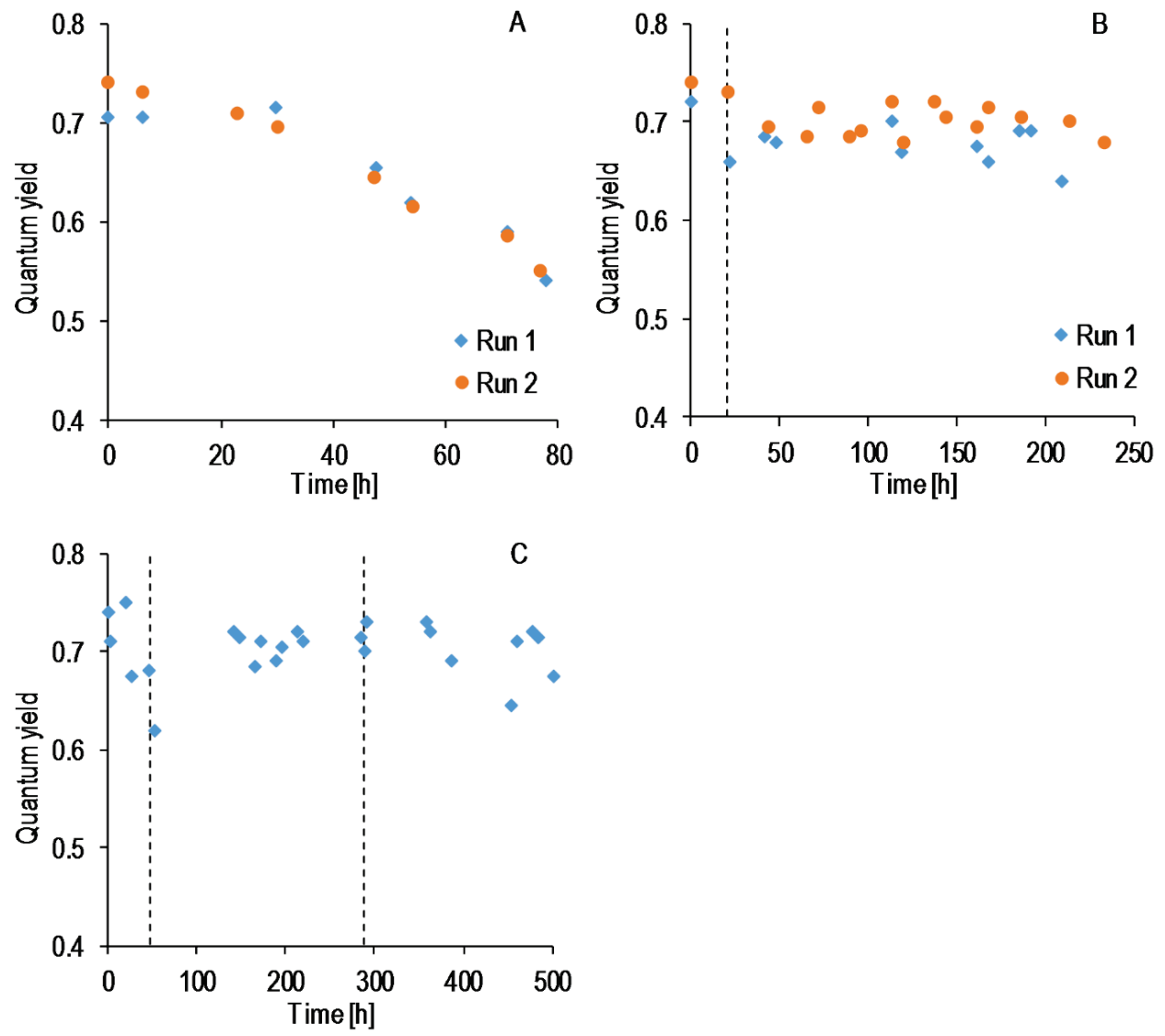

Figure 4.C.1. PSII Quantum yield of microalgal cells during cultivation in a liquid foam-bed photobioreactor. A) C. sorokiniana, batch reactor operation (see Figure 4.5 in main text), B) C. sorokiniana, chemostat reactor operation (see Figure 4.6 in main text), C) C. vulgaris, chemostat reactor operation (see Figure 4.7 in main text). The dashed line in B) and the first dashed line in C) indicate the end of the batch phase, while the second dashed line in C) indicate the change of operational conditions. 


\section{Appendix 4.D: Dynamics of gas supply, pH and Temperature during growth experiments}

The $\mathrm{pH}$, temperature and the relative time period of gas supply during all microalgae cultivation experiments are presented in Figure 4.D.1, 4.D.2 and 4.D.3.
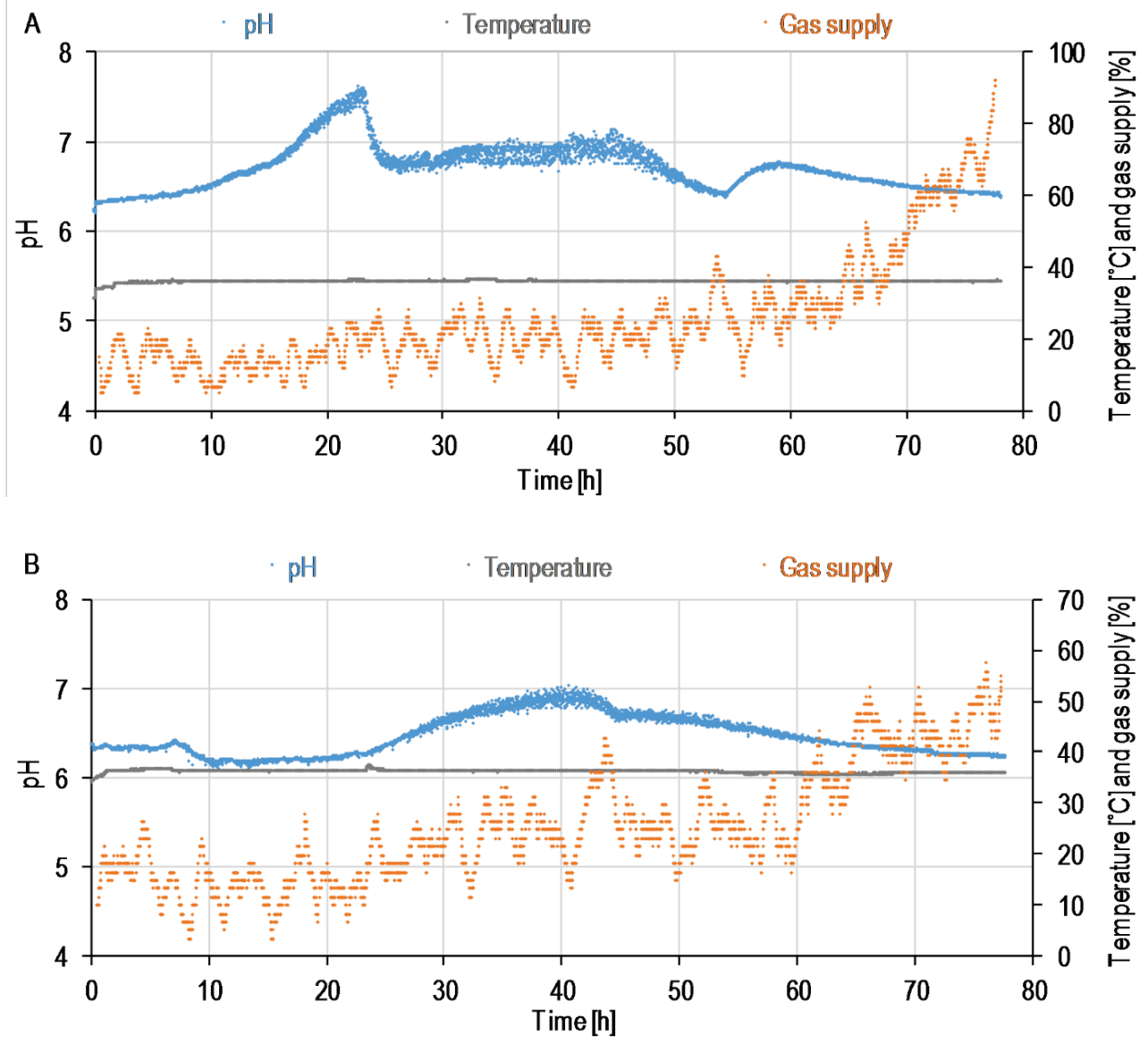

Figure 4.D.1. pH, temperature and the dynamics of gas supply for batch operation of $C$. sorokiniana (see Figure 4.5 in main text). The 'gas supply' is the percentage of the total operational time that gas was supplied to the reactor, presented as 1-hour moving average. A) Run 1, B) Run 2. 

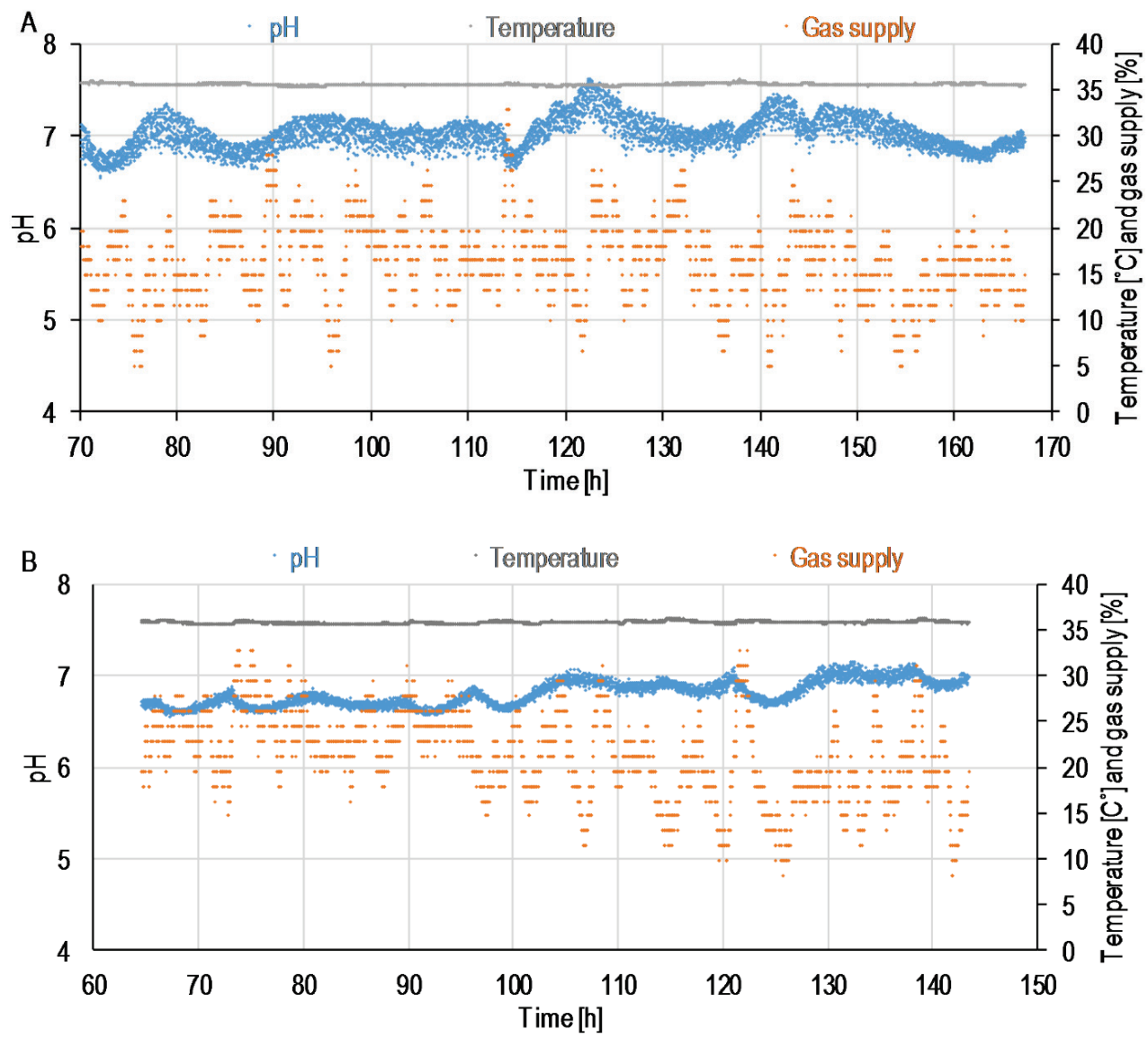

Figure 4.D.2. pH, temperature and the dynamics of gas supply for the steady state of the chemostat operation of the liquid foam-bed photobioreactor cultivating $C$. sorokiniana (see Figure 4.6 in main text). The 'gas supply' is the percentage of the total operational time that gas was supplied to the reactor, presented as 1 -hour moving average. A) Run 1, B) Run 2. 


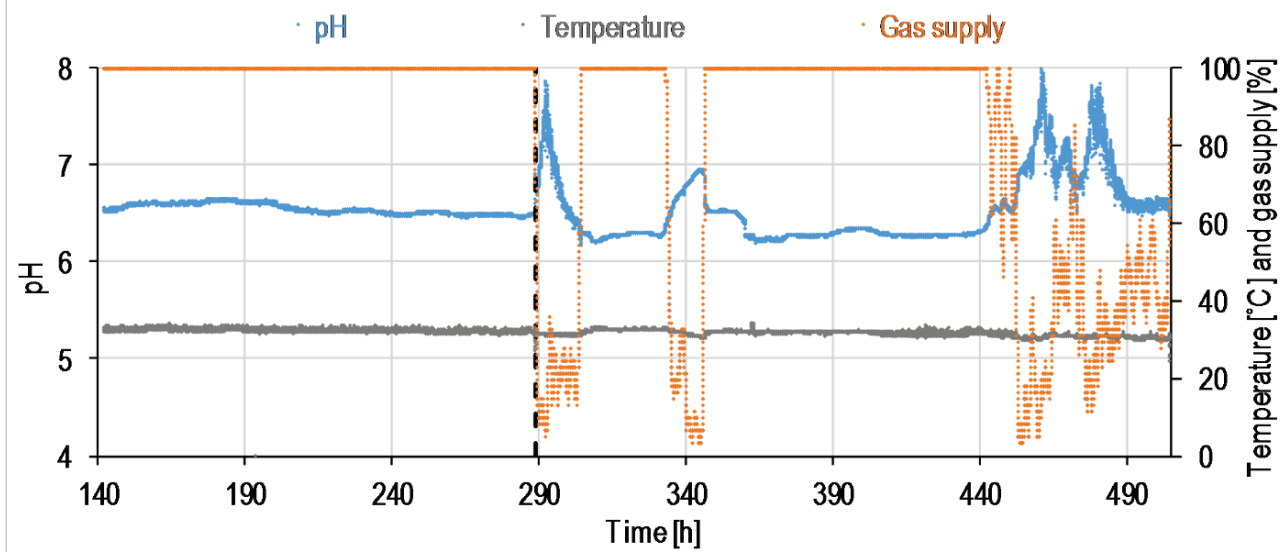

Figure 4.D.3. $\mathrm{pH}$, temperature and the dynamics of gas supply for the steady state of the chemostat operation of the liquid foam-bed photobioreactor cultivating $C$. vulgaris (see Figure 4.7 in main text). The 'gas supply' is the percentage of the total operational time that gas was supplied to the reactor, presented as 1 -hour moving average. The dashed vertical line represents the change in operational conditions. During this experiment, a manual $\mathrm{pH}$ control was applied: $0.1 \mathrm{M} \mathrm{NaOH}$ was added to the culture occasionally to restore the $\mathrm{pH}$. 


\section{Appendix 4.E: Surfactant concentration}

During the first chemostat experiment with C. sorokiniana, the Pluronic F68 concentration was determined in the reactor samples. To measure the surfactant concentration in the medium, a colorimetric assay was performed [55]. Biomass was removed from the reactor samples by centrifugation, and the supernatant fractions were stored at $4{ }^{\circ} \mathrm{C}$ until analysis. During analysis $250 \mu 1$ sample was mixed with $25 \mu 1$ trichloroacetic acid and $250 \mu 1$ methanol and vortexed for $30 \mathrm{sec}$. Afterwards, it was centrifuged at $12000 \mathrm{x} \mathrm{g}$, and the supernatant was transferred to a new tube with $250 \mu \mathrm{l}$ cobalt thiocyanate reagent and $250 \mu \mathrm{l}$ ethyl acetate. The cobalt thiocyanate reagent contained $200 \mathrm{~g} \mathrm{~L}^{-1}$ ammonium thiocyanate and $30 \mathrm{~g} \mathrm{~L}^{-1}$ cobalt nitrate hexahydrate. The mix was vortexed for $30 \mathrm{sec}$ and centrifuged at $12000 \mathrm{x}$ g for $5 \mathrm{~min}$. The supernatant was carefully discarded. The pellet was washed with $1 \mathrm{ml}$ ethyl acetate and subsequently centrifuged at $12000 \mathrm{x} \mathrm{g}$ for $5 \mathrm{~min}$. This step was repeated three times after which the pellet was air dried. Finally the dried pellet was dissolved in $1 \mathrm{ml}$ acetone. The absorbance was measured in a spectrophotometer (HACH DR6000) at $624 \mathrm{~nm}$ using a quartz cuvette. Pure acetone was used for blank measurements. A calibration curve produced by dissolving Pluronic F68 in medium was used to convert the obtained absorbance to Pluronic F68 concentration.

The growth medium contained 0.52 CMC Pluronic F68 according to our analysis. Already at the time of first sampling, the surfactant concentration in the reactor dropped to half of this initial concentration (Figure 4.E.1). This reduction of the concentration in the liquid phase is most likely related to the surfactant characteristic of Pluronic as it will concentrate on the gasliquid interface in the foam-bed. Therefore, the liquid layer underneath the foam column was depleted from surfactant molecules while the foam phase had increased surfactant concentrations [56].

The Pluronic F68 concentration decreased further until the end of the batch phase of the continuous cultivation experiment. The surfactant concentration was only $0.06 \mathrm{CMC}$ at 20 hours. At this point, increased bubble sizes and decreased liquid fraction was also observed visually. When the chemostat mode was initiated the surfactant concentration became stable around $0.15 \mathrm{CMC}$ because of the continuous supply of surfactants.

The observed surfactant concentration decrease at the end of the batch phase might be due to surfactant absorption or degradation. Since biodegradation of the molecule in 20 days is unlikely [5], chemical or thermal degradation, or absorption to the reactor walls or to the 
microalgal cells could have occurred. This observed decrease in Pluronic F68 concentration explains the decreased foam stability observed at the end of the three days batch cultivation of C. sorokiniana.

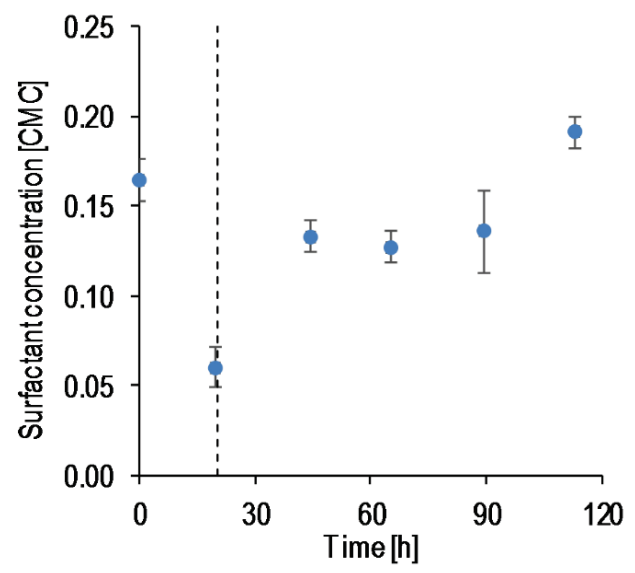

Figure 4.E.1. Pluronic F68 concentration in the reactor, expressed in terms of the CMC values of the surfactant, during the first chemostat reactor experiment with $C$. sorokiniana (Run 1). The growth medium contained $0.52 \mathrm{CMC}$ Pluronic F68. The dashed line represents the start of the continuous operation. The error bars show the deviations from the average value for the duplicate measurements. 


\section{References}

[1] R. Singh, S. Sharma, Development of suitable photobioreactor for algae production-A review, Renew. Sust. Energ. Rev. 16 (2012) 2347-2353.

[2] P. Spolaore, C. Joannis-Cassan, E. Duran, A. Isambert, Commercial applications of microalgae, J. Biosci. Bioeng. 101 (2006) 87-96.

[3] C. Posten, Design principles of photo-bioreactors for cultivation of microalgae, Eng. Life Sci. 9 (2009) 165-177.

[4] A. Janoska, P.P. Lamers, A. Hamhuis, Y. van Eimeren, R.H. Wijffels, M. Janssen, A liquid foam-bed photobioreactor for microalgae production, Chem. Eng. J. 313 (2017) 1206-1214.

[5] A. Janoska and M. Vázquez, R.H. Wijfels, M. Cuaresma, C. Vílchez, Surfactant selection for a liquid foambed photobioreactor, Biotechnol. Prog. DOI: 10.1002/btpr.2614

[6] M.Y. Kozlov, N.S. Melik-Nubarov, E.V. Batrakova, A.V. Kabanov, Relationship between pluronic block copolymer structure, critical micellization concentration and partitioning coefficients of low molecular mass solutes, Macromolecules 33 (2000) 3305-3313.

[7] I.R. Schmolka, A review of block polymer surfactants, J. Am. Oil Chem. Soc. 54 (1977) 110-116.

[8] S. Fusco, A. Borzacchiello, P. Netti, Perspectives on: PEO-PPO-PEO triblock copolymers and their biomedical applications, J. Bioact. Compat. Polym. 21 (2006) 149-164.

[9] Y. Chisti, Animal-cell damage in sparged bioreactors, Trends Biotechnol. 18 (2000) 420-432.

[10] P.M. Ireland, G.J. Jameson, Liquid transport in a multi-layer froth, J. Colloid Interface Sci. 314 (2007) 207213.

[11] P. Ireland, R. Cunningham, G.J. Jameson, The behaviour of wash water injected into a froth, Int. J. Miner. Process. 84 (2007) 99-107.

[12] P. Stevenson, Hydrodynamic theory of rising foam, Miner. Eng. 20 (2007) 282-289.

[13] N. Isert, G. Maret, C.M. Aegerter, Coarsening dynamics of three-dimensional levitated foams: From wet to dry, Eur. Phys. J. E 36 (2013) 116.

[14] A.-L. Biance, A. Delbos, O. Pitois, How topological rearrangements and liquid fraction control liquid foam stability, Phys. Rev. Lett. 106 (2011) 068301.

[15] E. Kan, M.A. Deshusses, Modeling of a foamed emulsion bioreactor: I. Model development and experimental validation, Biotechnol. Bioeng. 99 (2008) 1096-1106.

[16] D.C. Perry, P. Stevenson, Gas absorption and reaction in a wet pneumatic foam, Chem. Eng. Sci. 126 (2015) 177-185.

[17] A. Bhaskarwar, R. Kumar, Oxidation of sodium sulphide in a foam bed contactor, Chem. Eng. Sci. 39 (1984) 1393-1399.

[18] A.N. Bhaskarwar, R. Kumar, Oxidation of sodium sulphide in the presence of fine activated carbon particles in a foam bed contactor, Chem. Eng. Sci. 41 (1986) 399-404.

[19] M.U. Vera, A. Saint-Jalmes, D.J. Durian, Scattering optics of foam, Appl. Opt. 40 (2001) 4210-4214.

[20] E. Kan, M.A. Deshusses, Continuous operation of foamed emulsion bioreactors treating toluene vapors, Biotechnol. Bioeng. 92 (2005) 364-371.

[21] S. Takesono, M. Onodera, K. Yamagiwa, A. Ohkawa, Design and operation of rotating-disk foam-breakers fitted to tower fermenters, J. Chem. Technol. Biotechnol. 57 (1993) 237-246.

[22] M. Janssen, R. Wijfels, U. von Stockar, Biocalorimetric monitoring of photoautotrophic batch cultures, Thermochim. Acta 458 (2007) 54-64.

[23] E.H. Murchie, T. Lawson, Chlorophyll fluorescence analysis: a guide to good practice and understanding some new applications, J. Exp. Bot. 64 (2013) 3983-3998.

[24] L. Poughon, D. Duchez, J. Cornet, C. Dussap, kLa determination: comparative study for a gas mass balance method, Bioprocess Biosyst. Eng. 25 (2003) 341-348.

[25] J. Thibault, A. Leduy, A. Denis, Chemical enhancement in the determination of kLa by the sulfite oxidation method, Can. J. Chem. Eng. 68 (1990) 324-326.

[26] P. Parupudi, C. Kethineni, P.B. Dhamole, S. Vemula, P.R. Allu, M. Botlagunta, S. Kokilagadda, S.R. Ronda, CO2 fixation and lipid production by microalgal species, Korean J. Chem. Eng. 33 (2016) 587-593.

[27] W. Lu, H. Guo, I.-M. Chou, R. Burruss, L. Li, Determination of diffusion coefficients of carbon dioxide in water between 268 and $473 \mathrm{~K}$ in a high-pressure capillary optical cell with in situ Raman spectroscopic measurements, Geochim. Cosmochim. Acta 115 (2013) 183-204.

[28] P. Han, D.M. Bartels, Temperature dependence of oxygen diffusion in $\mathrm{H} 2 \mathrm{O}$ and D 2 O, J. Phys. Chem. 100 (1996) 5597-5602. 
[29] R. Reyna-Velarde, E. Cristiani-Urbina, D.J. Hernández-Melchor, F. Thalasso, R.O. Cañizares-Villanueva, Hydrodynamic and mass transfer characterization of a flat-panel airlift photobioreactor with high light path, Chem. Eng. Process.: Process Intensification 49 (2010) 97-103.

[30] S.K. Jana, A.N. Bhaskarwar, Modeling gas absorption accompanied by chemical reaction in bubble column and foam-bed slurry reactors, Chem. Eng. Sci. 65 (2010) 3649-3659.

[31] J.Y. Jing, J. Feng, W.Y. Li, Carrier effects on oxygen mass transfer behavior in a moving-bed biofilm reactor, Asia-Pac. J. Chem. Eng. 4 (2009) 618-623.

[32] J. Song, Y. Kim, Y. Son, J. Khim, A bioactive foam reactor for the removal of volatile organic compounds: system performance and model development, Bioprocess Biosyst. Eng. 30 (2007) 439-446.

[33] C.J. Hulatt, D.N. Thomas, Productivity, carbon dioxide uptake and net energy return of microalgal bubble column photobioreactors, Bioresour. Technol. 102 (2011) 5775-5787.

[34] H.C. Cheng, R. Lemlich, Errors in the measurement of bubble size distribution in foam, Ind. Eng. Chem. Fundam. 22 (1983) 105-109.

[35] R. Shaw, G.M. Evans, P. Stevenson, Start-up transients in a pneumatic foam, Asia-Pac. J. Chem. Eng. 6 (2011) 613-623.

[36] W.-D. Deckwer, R. Burckhart, G. Zoll, Mixing and mass transfer in tall bubble columns, Chem. Eng. Sci. 29 (1974) $2177-2188$.

[37] J. Vasconcelos, J. Rodrigues, S. Orvalho, S. Alves, R. Mendes, A. Reis, Effect of contaminants on mass transfer coefficients in bubble column and airlift contactors, Chem. Eng. Sci. 58 (2003) 1431-1440.

[38] J.M. Vasconcelos, S.P. Orvalho, S.S. Alves, Gas-liquid mass transfer to single bubbles: effect of surface contamination, AIChE J. 48 (2002) 1145-1154.

[39] A. Schumpe, W.D. Deckwer, Gas holdups, specific interfacial areas, and mass transfer coefficients of aerated carboxymethyl cellulose solutions in a bubble column, Ind. Eng. Chem. Process Des. Dev. 21 (1982) 706-711.

[40] M. Jamnongwong, K. Loubiere, N. Dietrich, G. Hébrard, Experimental study of oxygen diffusion coefficients in clean water containing salt, glucose or surfactant: Consequences on the liquid-side mass transfer coefficients, Chem. Eng. J. 165 (2010) 758-768.

[41] T. Solomon, The definition and unit of ionic strength, J. Chem. Educ. 78 (2001) 1691-1692.

[42] C. Sorokin, Tabular comparative data for the low-and high-temperature strains of Chlorella, Nature 184 (1959) 613-614.

[43] P. Duboc, I. Marison, U. Von Stockar, Quantitative calorimetry and biochemical engineering, in: R.B. Kemp (Ed), Handbook of thermal analysis and calorimetry: from macromolecules to man, Vol. 4, Elsevier, Amsterdam, 1999, pp. 267-365.

[44] G. Benvenuti, R. Bosma, M. Cuaresma, M. Janssen, M.J. Barbosa, R.H. Wiffels, Selecting microalgae with high lipid productivity and photosynthetic activity under nitrogen starvation, J. Appl. Phycol. 27 (2015) 1425-1431. [45] N.T. Eriksen, F.K. Riisgård, W.S. Gunther, J.J.L. Iversen, On-line estimation of O2 production, CO2 uptake, and growth kinetics of microalgal cultures in a gas-tight photobioreactor, J. Appl. Phycol. 19 (2007) 161-174.

[46] G. Breuer, L. de Jaeger, V.G. Artus, D.E. Martens, J. Springer, R.B. Draaisma, G. Eggink, R.H. Wijffels, P.P. Lamers, Superior triacylglycerol (TAG) accumulation in starchless mutants of Scenedesmus obliquus:(II) evaluation of TAG yield and productivity in controlled photobioreactors, Biotechnol. Biofuels 7 (2014).

[47] Y. Zheng, T. Li, X. Yu, P.D. Bates, T. Dong, S. Chen, High-density fed-batch culture of a thermotolerant microalga Chlorella sorokiniana for biofuel production, Appl. Energy 108 (2013) 281-287.

[48] J. Ruiz, P. Álvarez-Díaz, Z. Arbib, C. Garrido-Pérez, J. Barragán, J. Perales, Performance of a flat panel reactor in the continuous culture of microalgae in urban wastewater: prediction from a batch experiment, Bioresour. Technol. 127 (2013) 456-463.

[49] D. Durian, D. Weitz, D. Pine, Multiple light-scattering probes of foam structure and dynamics, Science 252 (1991) 686-688.

[50] V.D.-M.Ž. Barbarić-Mikočević, K. Itrić, Kubelka-Munk theory in describing optical properties of paper (I), Tech. Gazette, 18 (2011) 117-124.

[51] K. Tuantet, Microalgae cultivation for nutrient recovery from human urine, Environmental Technology, Wageningen University, Wageningen (2015) pp. 68-89.

[52] M. Cuaresma, M. Janssen, C. Vilchez, R.H. Wijffels, Productivity of Chlorella sorokiniana in a short light-path (SLP) panel photobioreactor under high irradiance, Biotechnol. Bioeng. 104 (2009) 352-359.

[53] J.C. Ogbonna, H. Tanaka, Night biomass loss and changes in biochemical composition of cells during light/dark cyclic culture of Chlorella pyrenoidosa, J. Ferment. Bioeng. 82 (1996) 558-564. 
[54] T. de Mooij, Z.R. Nejad, L. van Buren, R.H. Wijfels, M. Janssen, Effect of photoacclimation on microalgae mass culture productivity, Algal Res. 22 (2017) 56-67.

[55] H.H. Chung, C. Zhou, H.K. Khor, J. Quu, Direct determination of residual Pluronic F-68 in in-process samples from monoclonal antibody preparations by high performance liquid chromatography, J. Chromatogr. A 1218 (2011) 2106-2113.

[56] J. Boos, W. Drenckhan, C. Stubenrauch, On how surfactant depletion during foam generation influences foam properties, Langmuir 28 (2012) 9303-9310. 


\section{Chapter 5}

\section{Potential of a liquid foam-bed photobioreactor for microalgae cultivation}

This chapter is submitted for publication as:

A. Janoska, V. Andriopoulos, R. H. Wijffels, M. Janssen, Potential of a liquid foam-bed photobioreactor for microalgae cultivation. 


\begin{abstract}
The liquid foam-bed photobioreactor is a novel photobioreactor for microalgae cultivation. A mathematical model was developed to evaluate its potential, and to optimize the design and operation of a large-scale unit. This model describes light limited microalgal growth in a rising foam column in a foam-bed photobioreactor, which is continuously operated at constant biomass density. The microalgae-containing liquid is recirculated from the bottom of the reactor and dispersed equally on the top of the foam column, in order to ensure homogenous microalgae distribution and a wet and stable foam. The model combines calculations of liquid fraction gradient, light penetration, microalgal growth, and gas transfer in the foam-bed. The liquid fraction and light model was experimentally validated. The areal productivity of a $5 \mathrm{~cm}$ deep foam-bed photobioreactor operated at $30 \mathrm{~g} \mathrm{~L}^{-1}$ microalgae and $1500 \mu \mathrm{mol}$ photons $\mathrm{m}^{-2} \mathrm{~s}^{-1}$ was estimated to be $67.7 \mathrm{~g} \mathrm{~m}^{-2} \mathrm{~d}^{-1}$. This productivity is slightly lower compared to what is achievable in flat panels, which is related to light scattering in the foam-bed. Nevertheless, the advantages of the foam-bed photobioreactor, such as high gas transfer rate and high biomass densities, were confirmed with the simulations. In addition, it was calculated that a $\mathrm{CO}_{2}$ uptake efficiency of $97 \%$ can be obtained ensuring minimal $\mathrm{CO}_{2}$ loss. These benefits result in reduced gas supply requirement and reduced energy required for downstream processing. The total energy required for the production and separation of $1 \mathrm{~g}$ biomass in liquid foam-beds is only $8.5 \%$ of what is required in flat panels with suspended biomass. These results highlight the potential of foambed photobioreactors for large scale application for microalgae production.
\end{abstract}




\subsection{Introduction}

In order to produce microalgal biomass at reduced costs and energy requirements, the importance of photobioreactor design has been highlighted in several studies [1-3]. A liquid foam-bed photobioreactor is an alternative photobioreactor concept to existing liquid-phase photobioreactors [4]. This reactor configuration was developed in order to reduce the energy requirements related to harvesting and gas supply. The reduction in harvesting costs is based on increased biomass densities due to the short light absorption length in the photobioreactor. The improved mass transfer is related to the increased surface area between the gas and the liquid phase, and the increased contact time between the $\mathrm{CO}_{2}$-enriched gas and the microalgaecontaining liquid. Additionally, reduced energy requirement for the gas supply is due to the reduced pressure drop in the reactor since the density of the foam is reduced compared to liquid. These presumed advantages, however, require exact characterization of the physical, chemical, and biological phenomena taking place in the reactor. Besides, in order to quantify the possible energy savings, the algal growth in the foam-bed photobioreactor has to be numerically evaluated. Therefore, a model predicting the productivity and energy requirements of a foambed photobioreactor was developed.

The operation of a foam-bed photobioreactor is based on continuous gas supply to a shallow layer of microalgae and surfactant containing solution. Due to the presence of surfactants, bubbles are formed, which will rise until the top of the reactor. In order to refresh the incorporated gas, the foam bubbles have to be broken. A possible method is to externally break the bubbles in a foam breaker device, as described for a previous foam-bed design [4]. Alternatively, the foam can be broken inside the reactor at the top. Due to the internal foam breaking the liquid fraction of the foam is enhanced as a result of the extra liquid drainage resulting from the liquid flux deliberated from broken bubbles [5]. A practically convenient solution is to break the foam with water jets. These water jets can originate from the microalgaeenriched liquid inside the reactor. Therefore, a liquid recirculation can be introduced, where the liquid from the bottom of the reactor is lead externally via pumps to the top where it is equally distributed over the foam column. This liquid drains down again in the foam column. Figure 5.1 illustrates this improved foam-bed photobioreactor design including liquid recirculation and internal foam breaking. Due to the liquid recirculation and the internal foam breaking, the liquid fraction of the foam is enhanced. Enhanced liquid fraction in the foam is advantageous, since it produces a more stable foam [5], since both coalescence and coarsening are reduced [6,7]. In 
thin reactors or high light intensities, this elevated liquid fraction is also beneficial to absorb all incident light by increasing the light absorption path (i.e. more algae behind the 'window') resulting in increased productivity. The proof of principle of such a system has been confirmed at laboratory scale [8].

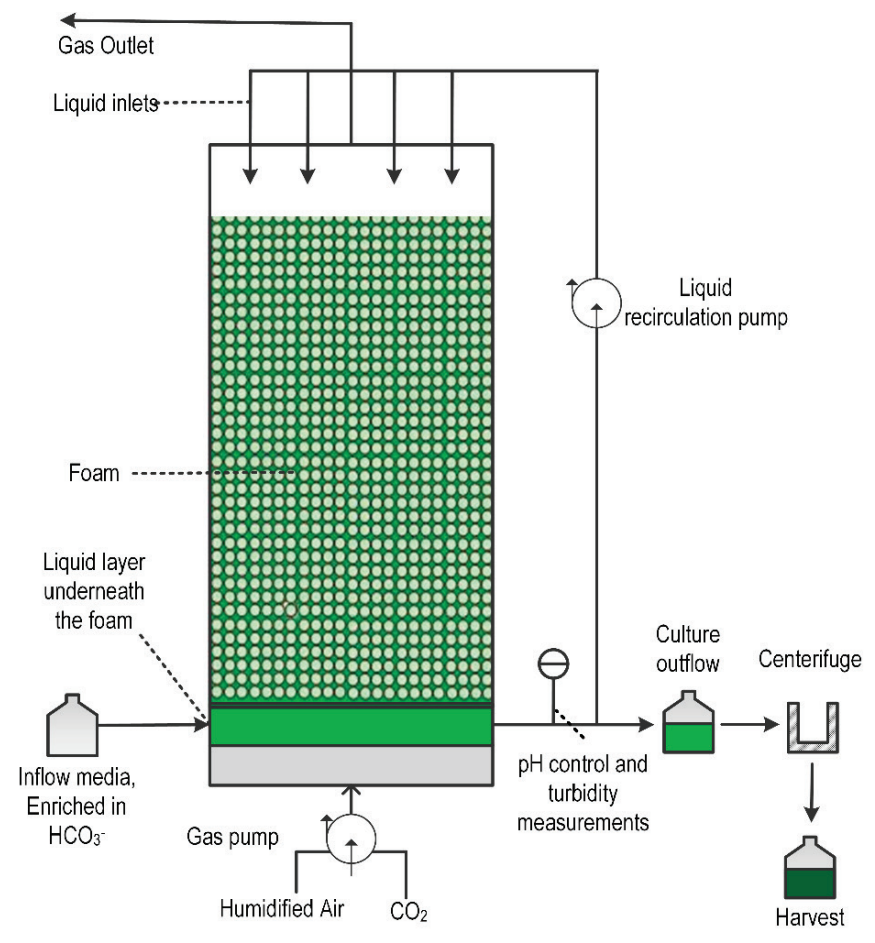

Figure 5.1. Scheme of the liquid foam-bed photobioreactor modelled. The design of the foam-bed is improved compared to the previous design [4]: internal foam breaking and liquid recirculation has been introduced.

To create foam, Pluronic F68 was found to be a suitable surfactant in the liquid foam-bed photobioreactor. Despite its good foaming properties, lack of toxicity, and low biodegradability, microalgal partitioning to the foam phase formed by Pluronic F68 is reduced. Therefore, the microalgal concentration in the liquid content of the foam is lower compared to the liquid layer below the foam [9]. The liquid recirculation also circumvents this problem and ensures equal algal distribution in the foam, thereby resulting in an elevated microalgae concentration in the foam.

The model developed in this study describes a flat panel foam-bed photobioreactor with liquid recirculation and homogeneous liquid distribution on the top of the foam column. The reactor was operated in continuous mode: the biomass concentration does not change in time once a 
steady state is reached. The growth of a model microalgae strain, Chlorella sorokiniana was described. The developed model provides insight into the most important biological, chemical, and physical phenomena linked to the operation of foam-bed photobioreactors. The aim of the model is to determine the potential of the foam-bed photobioreactor and compare its potential productivity and energy requirement to conventional photobioreactors, e.g. flat panels. Next to that, the model aids further improvement in system design and operation. To achieve this, the influence of several operational parameters were investigated. Experimental validation took place for the core elements of the model (liquid fraction and light penetration).

\subsection{Model structure, calculations, and assumptions}

We modelled a flat panel photobioreactor filled with foam, under continuous illumination. The biological parameters were based on Chlorella sorokiniana CCAP 211/8k, a very productive species thoroughly studied in our laboratory [10]. A turbidostat operational mode was considered and, consequently, the biomass concentration $\left(C_{x}\right)$ in the system can be considered to be constant throughout the operation. In practice, turbidity can be continuously measured in the liquid layer underneath the foam or in the recirculated liquid phase. The corresponding reactor dilution rates are equal to the algal specific growth rates, which depend again on the value of $C_{x}$. Time changes were therefore not considered in the mass balances applied in this study. In the model we consider a constant $\mathrm{pH}$ of 6.7 throughout the whole foam column. In order to avoid $\mathrm{CO}_{2}$ limitation, the required $\mathrm{CO}_{2}$ concentrations were calculated and a constant gas flow rate was assumed.

In the simulations both design and operational parameters are varied in order to predict productivity and energy requirements of large scale foam-bed photobioreactors of different dimensions and under different operational conditions. The effect of biomass concentration, light levels, gas flow rate, liquid recirculation rate, bubble size, and reactor depth was determined on biomass productivity and operational energy requirements. As a baseline, a reactor of $2 \mathrm{~m}$ height, $1 \mathrm{~m}$ width and $5 \mathrm{~cm}$ depth was considered, operated under $1500 \mu \mathrm{mol} \mathrm{m}$ ${ }^{2} \mathrm{~s}^{-1}$ incident light intensity, a biomass concentration of $30 \mathrm{~g} \mathrm{~L}^{-1}$, a gas flow rate of $0.5 \mathrm{~mm} \mathrm{~s}^{-1}$, and a liquid recirculation rate of $0.052 \mathrm{~mm} \mathrm{~s}^{-1}$. Although a width of $1 \mathrm{~m}$ was considered to define a 'unit', the model aims to simulate a $1 \mathrm{~m}$ wide block of a large scale reactor with infinite width. The applied values are presented in Table 5.1. 
Table 5.1. Simulated design and operational conditions for the foam-bed photobioreactor.

\begin{tabular}{lccccc}
\hline Variable & Notation & \multicolumn{3}{c}{ Values } & Unit \\
\hline Biomass concentration & $C_{x}$ & 15 & 30 & 60 & $\mathrm{~g} \mathrm{~L}^{-1}$ \\
Light intensity & $E_{0}$ & 375 & 750 & 1500 & $\mu \mathrm{mol} \mathrm{m}^{-2} \mathrm{~s}^{-1}$ \\
Gas superficial velocity & $J_{G}$ & 0.05 & 0.5 & 2.5 & $\mathrm{~mm} \mathrm{~s}^{-1}$ \\
Liquid superficial velocity & $J_{W}$ & 0.026 & 0.052 & 0.104 & $\mathrm{~mm} \mathrm{~s}^{-1}$ \\
Bubble size & $r_{b}$ & 0.5 & 1 & 2 & $\mathrm{~mm}$ \\
Depth & $d$ & 0.01 & 0.5 & 0.1 & $\mathrm{~m}$ \\
\hline
\end{tabular}

The model structure is presented in Figure 5.2. Firstly the liquid fraction of the foam column is calculated as a function of height $(\varepsilon)$. The liquid fraction profile is based on the physicalchemical properties of the liquid phase containing surfactant and microalgae, the gas flow rate, and the liquid recirculation flow rate. Most existing models describing foam-bed reactors do not consider the liquid fraction gradient, but assume a constant liquid fraction in height [11]. The description of this gradient in this study allows for more precise calculations. This liquid fraction profile influences the optical properties of the foam at different heights: at higher liquid fractions more microalgae are present, and also the scattering properties of the foam will be influenced. Based on the optical properties of the foam, the local light availability inside the foam-bed can be predicted. The light penetration in the foam was modelled with the light diffusion theory. The foam has a highly scattering nature, thus at a certain position in the foam photons are arriving from all directions: in this case we talk about fluence rate $(\phi)$, owing the same units as irradiance $\left[\mathrm{Wm}^{-2}\right.$ or $\mu \mathrm{mol}$ photons $\left.\mathrm{m}^{-2} \mathrm{~s}^{-1}\right]$. From the local fluence rate availability, the microalgae growth rate $(\mu)$, the resulting biomass productivities (volumetric and areal productivity, $r_{x}$ and $r_{x}^{\text {Areal }}$, respectively) and biomass yield on light $\left(Y_{x, p h}\right)$ were calculated. The biomass production rate determines the $\mathrm{CO}_{2}$ requirements for the algal growth. With the gas transfer model, the $\mathrm{CO}_{2}$ concentration could be predicted that minimally needs to be supplied within the inlet gas phase $\left(\mathrm{C}_{\mathrm{CO} 2}{ }^{G, i n}\right)$. From the inlet and outlet $\mathrm{CO}_{2}$ concentrations the $\mathrm{CO}_{2}$ uptake efficiency, $\eta_{\mathrm{CO} 2}$ could be determined. In addition, the $\mathrm{O}_{2}$ accumulation in the liquid phase $\left(C_{O 2}{ }^{L}\right)$ could be calculated. The operational energy requirements $(E)$ were then calculated based on the energy requirements for gas supply by gas blowers, liquid recirculation by liquid pumps, and biomass separation by centrifuges. 


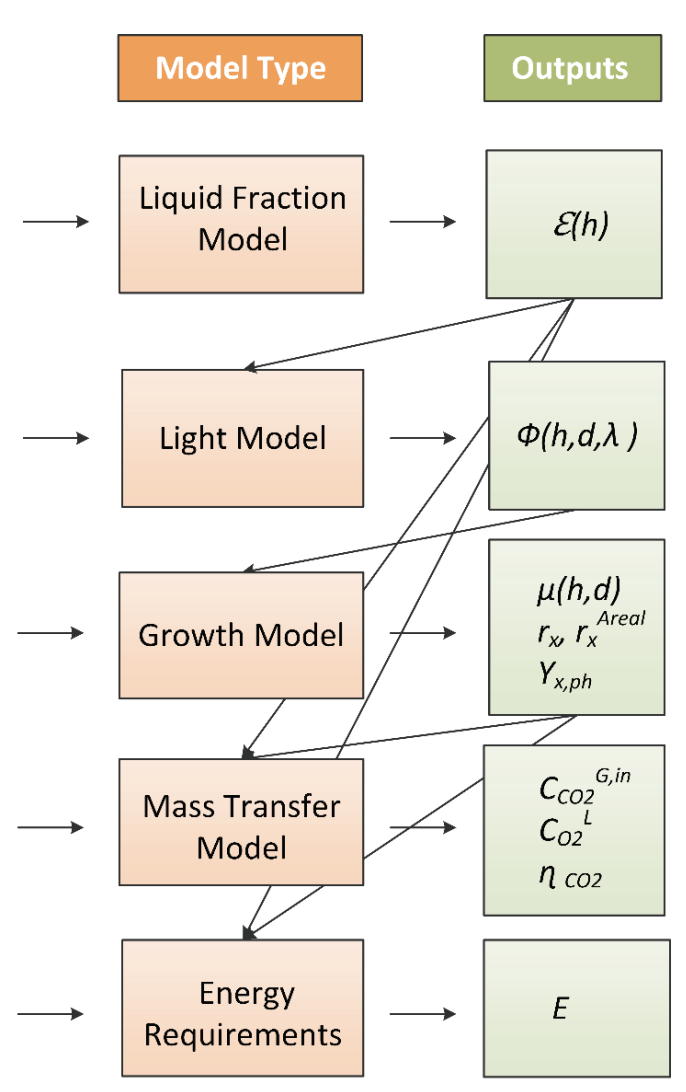

$J_{G}, r_{b}, J_{W}, h, d, w$
$\mu_{L}, \sigma, \rho, g, \varepsilon_{\text {max }} n_{1}-n_{4}, \alpha$

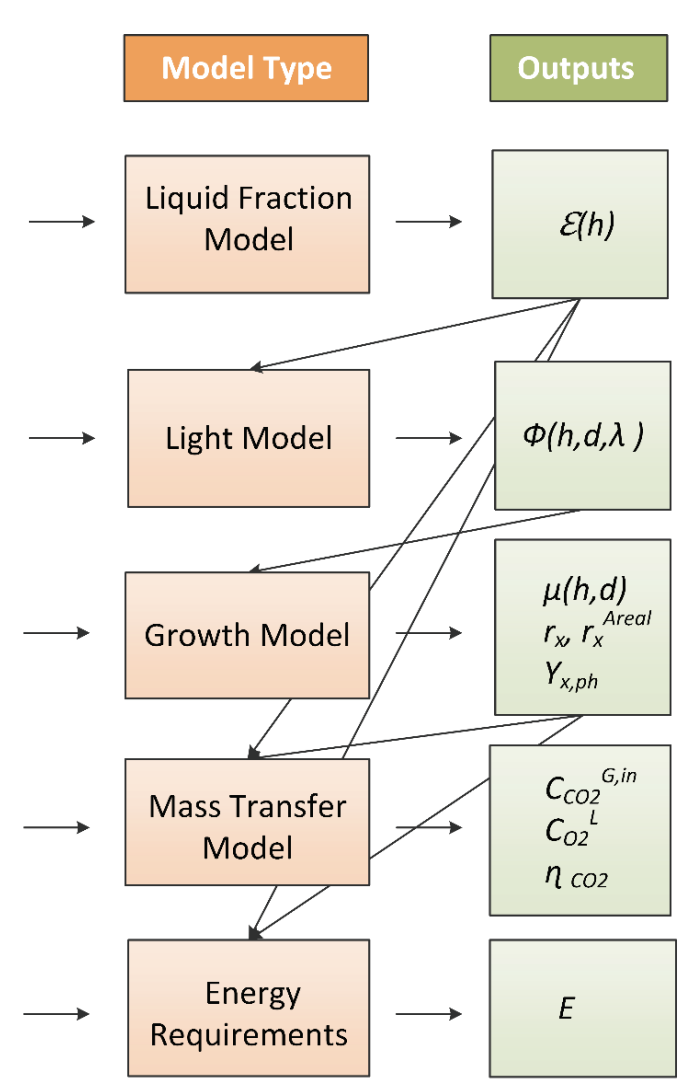

Figure 5.2. Scheme of the model describing the performance of foam-bed photobioreactors. The left column (blue rectangles) shows the model inputs, which are used to calculate the model outputs listed in the right column (green rectangles). The model outputs include liquid fraction as a function of height, $\varepsilon(h)$; fluence rate distribution, $\phi(h, d, \lambda)$; local growth rates, $\mu(h, d)$; volumetric and areal biomass productivities, $r_{x}$ and $r_{x}^{\text {Areal }}$, respectively; biomass yield on light $Y_{x, p h}$, inlet $\mathrm{CO}_{2}$ concentration, $\mathrm{C}_{\mathrm{CO}_{2}}{ }^{\mathrm{Gin}} ; \mathrm{O}_{2}$ concentration in the liquid phase $\mathrm{C}_{02}{ }^{2} ; \mathrm{CO}_{2}$ uptake efficiency $\left(\eta_{\mathrm{CO} 2}\right)$ and biomass specific energy requirements, $E$. Explanation of model inputs can be found in Appendix 5.B.

In the model, the following assumptions were applied:

- The bubbles are spherical, with uniform size (no expansion in height due to pressure differences or due to gas diffusion, no coalescence/coarsening/breakage). A decreasing liquid fraction therefore means an increasing number of gas bubbles in a given volume.

- The distribution of the recirculated liquid on the top of the foam column is homogenous.

- The liquid phase is ideally mixed. Most previous models on foam-reactors assume stagnant liquid layers, where mass transfer is solely via diffusion $[11,12]$, however these reactors did not apply liquid recirculation that enhance mixing. Ideally mixed liquid phase in case of liquid recirculation was reported previously [13]. This assumption is 
further supported by the reduced retention time of recirculated the liquid compared to gas phase of the foam-bed.

- The gas phase within each gas bubble is ideally mixed, similarly with previous foambed models [12], but individual gas bubbles do not interact.

- The liquid layer underneath the foam column has negligible volume. Therefore no gas transfer and no growth take place in this area. This assumption is made since in practice, the volume of this liquid layer is minimized.

- Liquid phase density, surface tension, viscosity were measured once for a specific biomass density and assumed to be constant for the different simulations with different biomass densities.

- The gas phase resistance is negligible, therefore only liquid phase gas transfer resistance $\left(k_{L}\right)$ is considered.

- Henry's law is applied for the dissolution of oxygen and carbon dioxide. We thus assume dilute solutions at low partial pressures. Solubility of oxygen and carbon dioxide is not corrected for increased ionic strength.

- Absolute pressure is assumed to be 1 atm at each position in the foam-bed, since the hydrostatic pressure of the foam column simulated is less than $10 \%$ of $2 \mathrm{~m}$ water column.

- All gases behave as ideal gasses.

- No loss of $\mathrm{H}_{2} \mathrm{O}$ by evaporation takes place because the gas is humidified before entering the foam-bed.

- No change in $\mathrm{pH}$ occurs across the foam-bed.

- Light escaping from the foam reactor top or bottom is neglected.

\subsubsection{Liquid fraction gradient in foams}

The liquid fraction is an important parameter of foams, determined by surfactant type and concentration, gas flow rate, design of gas distributor, and further physical-chemical properties of both the foamed solution (density, viscosity, surface tension) and of the formed foams (bubble size, rigidity of interfaces and dispersity of bubble size distribution) [14]. It is well known that the liquid fraction in rising foams is not constant: at the bottom, at the point of foam generation wet foam is present, while at the top, the foam is drier because of drainage of the liquid [5]. The liquid fraction gradient in height in a rising foam can be calculated according to Yazhgur et. al [14]. In case additional liquid is supplied to the top of the foam column, the liquid 
fraction of the foam is elevated due to the drainage of the supplied liquid through the foam-bed. To account for this, the theory of Yazhgur et. al can be supplemented by Stevenson's concept about liquid addition from the top [5].

The theory of Yazhgur is based on the calculation of the net superficial liquid velocity in rising foams, which can be obtained from the sum of the upward liquid movements and the relative downward liquid movements. The net superficial liquid velocity is constant through the foambed due to the conservation of mass. The upward liquid flux is caused by gas bubbles rising and dragging liquid along upwards. However, the liquid does not travel at the same speed as the gas bubbles due to drainage. This drainage is caused by gravity but also counteracted by capillary forces originating from the osmotic pressure gradient in height. This osmotic pressure is created by the difference between the energetic states of the interfilms originating from the varying bubble sphericity, which is determined by the liquid fraction of foams. Consequently, liquid drainage through the foam depends on liquid fraction gradient.

In a rising foam, the net liquid superficial velocity $\left(J_{f}\right)$ owns an upward direction since the foam volume is continuously increasing and the volume of the liquid on the bottom used to form foam is thus decreasing. In case of additional liquid supply to the top of the foam column, the net liquid superficial velocity might change orientation. Considering continuous foam breaking at a given height, the water liberated from the broken bubbles can be seen as an additional liquid flux. Thus, the net liquid superficial velocity when foam breaking is applied will be 0 , since the water addition rate resulting from foam breaking $\left(J_{w}^{f b}\right)$ equals to the net upward superficial velocity of a rising foam without foam breaking. In case both foam breaking and external liquid recirculation is applied, the net superficial velocity will be orientated downwards, and its value will equal to the water addition rate, $J_{f}^{w+f b}=-J_{w}$.

By knowing the steady state net liquid superficial velocity of the foam, the liquid fraction gradient in height of the foam can be expressed with the upward and downward liquid fluxes. A mathematical description of this theory can be found in Appendix 5.B.

\subsubsection{Light penetration in foams}

When a collimated beam hits the reactor surface, part of the light is reflected at the surface, while the remaining collimated light is attenuated in the foam-bed by algal absorption and scattering by the foam structure itself (caused by multiple reflections at the bubble liquid interface). By multiple scattering events, diffuse light is generated. Therefore, light propagation 
in the foam-bed photobioreactor was modelled assuming light diffusion theory, which is required due to the highly scattering nature of the foam. Consequently, the algal cells in the foam-bed do not only receive light from a single direction but photons can arrive from each possible direction. In this case we talk about fluence rate, owing the same units as irradiance $\left[\mathrm{Wm}^{-2}\right.$ or $\mu \mathrm{mol}$ photons $\left.\mathrm{m}^{-2} \mathrm{~s}^{-1}\right]$. Diffusion theory is a relatively simple method to model light propagation in a scattering medium although the accuracy of diffusion theory is reduced close to the system boundaries and the light source, and in situations where strong absorption takes place compared to scattering [15].

The path of collimated light is indicated with yellow arrows in Figure 5.3. The attenuation of collimated light due to scattering and absorption is modelled based on Lambert Beers law. The diffuse light generated also attenuates in forward and backward directions due to further absorption and scattering as indicated with orange arrows in Figure 5.3. Both the collimated and diffuse light are partly reflected at the transparent reactor sides. In this study a reactor with glass walls was considered, therefore reflections at air-glass and glass-liquid interfaces were taken into account.

The fluence rate can be predicted at each point in the reactor when knowing the reflection factors for both diffuse and collimated light at the reactor sides and the scattering and absorption coefficients of the foam with microalgae. The latter two are dependent on the liquid fraction, and thus on the height in the reactor. The scattering coefficient is further influenced by the foam bubble size, and the absorption coefficient is determined by the biomass concentration and the wavelength specific absorption cross section of the microalgal biomass. Scattering by the algal cells is not included in the model. For the simplicity of the light model, we did not consider the interaction of scattered light originating from different heights with different optical properties. Thus, the fluence rate at a certain height is only dependent on the liquid fraction at that given height and not on the liquid fraction in the layers below and above. This assumption proved to be acceptable because the calculated liquid fraction was constant over the largest part of the height. The liquid fraction was higher only over a few centimetres above the liquid level where foam is generated. Additionally the validity of light transport equations used in this study was confirmed by additional simulations using the diffusion approximation [15], which takes into account both the horizontal and vertical light spreading. The fluence rate distribution showed a perfect match with the diffusion approximation simulations under the simulation conditions, which might be reasoned by that when high biomass concentrations are applied and absorption increases, the influence of scattering from other layers is reduced. 


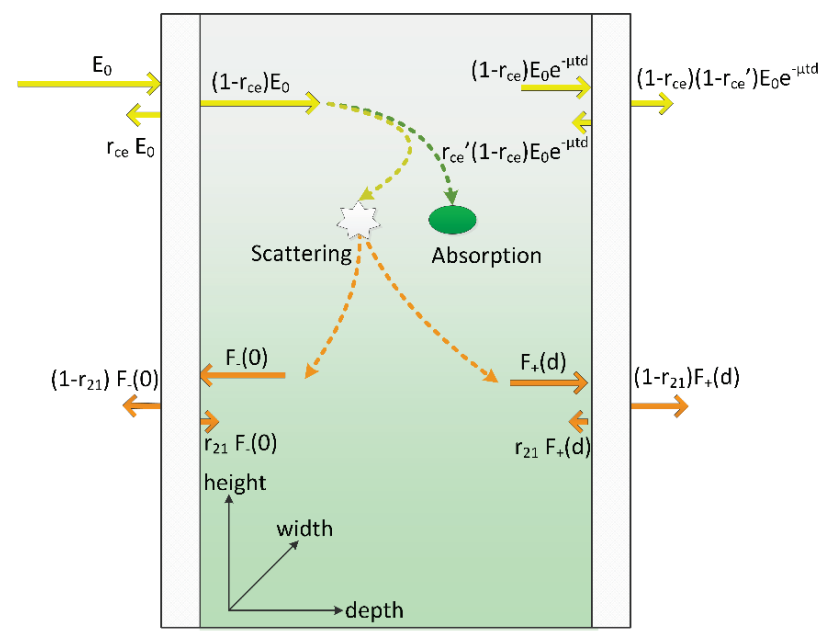

Figure 5.3. Light propagation in a liquid foam-bed. The incident collimated light beam is partially reflected at the surface of the reactor (yellow arrows indicate collimated light). Collimated light transmitted through the reactor wall is absorbed and scattered inside the foam and, consequently, it results in diffuse light going in forward and backward directions (indicated by orange arrows). Both the collimated and the diffuse light inside the foam is partially reflected at the surface of the reactor before a light beam leaves the reactor.

The theory for light propagation in an infinitely wide and high, but finitely thick slab (in depth), illuminated by wide-beam collimated light is considered [15]. The depth is defined in the direction of the incident collimated light beam. Since the reactor height is fixed at $2 \mathrm{~m}$, edge effects taking place near the reactor top and bottom are neglected. There are no boundaries at reactor sides which are parallel to the incoming light since a $1 \mathrm{~m}$ wide element of an infinitely wide reactor is considered in our simulations. The light model developed in this study is mathematically explained in Appendix 5.B. It predicts the fluence rate in the reactor at any position (thus at given height and depth coordinates) accounting for the spectral distribution of the incident light and also the spectral distribution of the absorption coefficient of the microalgal cells for wavelengths between 400 and $700 \mathrm{~nm}$ (wavelength specific values are presented in the online Supplementary material).

\subsubsection{Microalgae growth model}

The microalgae specific growth rate inside a liquid foam-bed photobioreactor was modelled based on the local fluence rate. For the growth, nitrogen is supplied in the form of urea. Algal growth is modelled according to Jassby \& Platt's model, where the algal growth rate is related to its sugar production rate, which is defined by the light availability [10]. The local fluence rates are determined by the previously described light model and it includes all photons within the photosynthetically active radiation (PAR) range, ranging from 400 to $700 \mathrm{~nm}$. Above a 
certain light intensity (or fluence rate), which is called the light saturation point, excess light is dissipated by the algal cells. At fluence rates below the light saturation point the microalgae specific growth rate is proportional to the fluence rate. Although mixing times in the foam-bed photobioreactor are in the order of several minutes, this is still considerably smaller than the characteristic time of photoacclimation which is in the order of hours. Photoacclimation can thus be neglected and a constant wavelengths-specific value is used for the microalgae-specific light absorption cross section, $a_{x}$. The value of $a_{x}$ was determined assuming a mass culture acclimated algal cell.

The local specific growth rates were averaged over the complete liquid volume of the foam-bed due to the assumption of an ideally mixed liquid phase. The resulting average specific growth rate is used to calculate the volumetric biomass production rate, areal productivity and biomass yield on light. The volumetric biomass production rate determines the $\mathrm{O}_{2}$ production and $\mathrm{CO}_{2}$ consumption rates. The corresponding equations are presented in Appendix 5.B.

\subsubsection{Gas transfer model}

The gas transfer in the foam is modelled by assuming an ideally mixed liquid phase, while the gas phase moves upwards in plug-flow (Figure 5.4). The plug flow behaviour of the gas was based on the fact that the gas bubbles are physically separated, so a gradient in height will occur. This gradient is characterised by a decreasing $\mathrm{CO}_{2}$ concentration and an increasing $\mathrm{O}_{2}$ concentration with height due to the transfer between gas bubbles and liquid with photosynthesising algae. An ideally mixed liquid phase is a simplification but is based on the calculation that the residence time of the recirculated liquid in the foam-bed is at least 3 times smaller than that of the gas phase (calculation based on model results presented later). This assumption is further supported by previous reports on a well-mixed zone in the foam below the liquid addition level [13]. Consequently, the liquid phase concentration of a certain compound is constant everywhere (no dependence on y and $\mathrm{z}$ ) whereas the liquid fraction $\varepsilon(y)$, the gas phase concentrations $C_{i}^{G}(y)$ and the transfer coefficients $k_{L} a(y)$ are dependent on the height of the reactor.

In the model calculations only the liquid phase mass transfer resistance was considered and the gas side limitations were neglected. The volumetric mass transfer coefficient, $k_{L} a\left[\mathrm{~s}^{-1}\right]$, was calculated from the liquid side mass transfer coefficient $k_{L}(y)$ and the surface area available for mass transfer per unit volume of foam, $a(y)$, which are both dependent on the liquid fraction of the foam. The $k_{L}(y)$ is calculated by a correlation for a packed-bed of solid spheres, which 
provides a good match with the $k_{L}(y)$ of foam-beds [16]. This correlation is based on the difference between gas and liquid velocities, determining the Reynolds number, and further dimensionless numbers (Schmidt and Sherwood numbers) related to diffusion coefficient, liquid phase properties and bubble size [16].

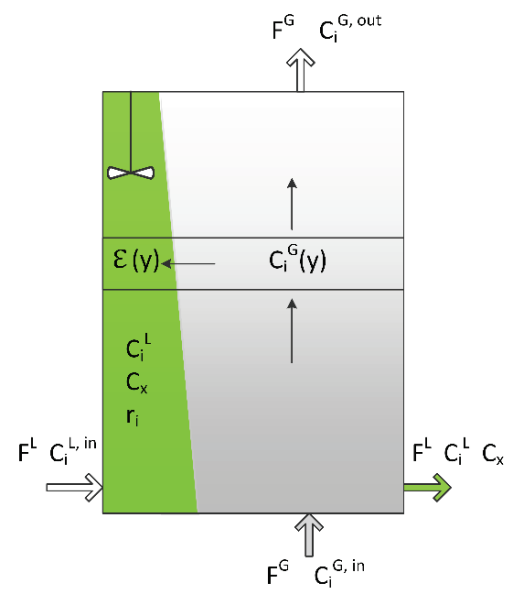

Figure 5.4. The gas transfer model of the foam-bed photobioreactor. The foam is modelled as a separate liquid and gas phase, with the interfacial area in between them related to the surface area of the bubbles at different heights. The decrease of the liquid fraction in height was modelled as an increase in the number of gas bubbles compared to liquid volume. The liquid phase was assumed to be ideally mixed while the gas moves upwards in plug-flow.

In order to avoid $\mathrm{CO}_{2}$ limitation and ensure light limited growth, the overall $\mathrm{C}_{\mathrm{CO}_{2}}{ }^{L}$ needs to be sufficiently high. This limit is arbitrarily set at $5 \cdot K_{s}$, where $K_{s}$ is the $\mathrm{CO}_{2}$ concentration where the growth rate is decreased to half of its maximal value. Thus, a minimum $\mathrm{CO}_{2}{ }^{G}$ (in) concentration in the inflow gas has to be determined that results in $C_{\mathrm{CO}_{2}}{ }^{L} \geq 5 \cdot K s$. With this given liquid $\mathrm{CO}_{2}$ concentration, the $\mathrm{CO}_{2}$ concentration required in the inlet gas can be calculated from a combination of mass balances and consequently the $\mathrm{CO}_{2}$ uptake efficiency can also be expressed. In the continuous liquid inflow, $\mathrm{HCO}_{3}{ }^{-}$is supplied at a concentration of $5 \cdot \mathrm{Ks}$. The liquid $\mathrm{O}_{2}$ concentration can be determined as well by the given inlet gas composition and the microalgal $\mathrm{O}_{2}$ production rate. In case of oxygen accumulation in the liquid phase, oxygen inhibition might take place [17] but this inhibition is not included in the model. The corresponding model equations are included in Appendix 5.B.

\subsubsection{Energy requirements}

The energy requirements for a foam-bed photobioreactor were calculated considering gas supply, liquid recirculation, and harvesting. The energy requirements for the gas supply by gas 
blowers or compressors were determined based on adiabatic compression of the gas. The pressure of the compressed gas has to equal to the hydrostatic pressure of the water column. An efficiency of $70 \%$ was applied as described for a rotary compressor [18]. For pumping liquid to higher levels the hydrostatic pressure and the volumetric recirculation flow rate was considered. The pump efficiency depends on pump design, and in this study an efficiency of $75 \%$ was used according to Ruiz et al. [2]. The energy requirement for the algal separation are determined by the biomass concentration and the method of harvesting. We considered harvesting by centrifugation and assumed a plate separator requiring $1.5 \mathrm{kWh}\left(5.4 \cdot 10^{6}\right.$ joules $)$ per $1 \mathrm{~m}^{3}$ of algal suspension to be separated [19].

\subsection{Materials and methods}

\subsubsection{Parameter determination}

The viscosity of M8a media, surfactant solution (10 CMC Pluronic F68 corresponding to 3.34 $\mathrm{g} \mathrm{L}^{-1}$ ), and microalgae cultures (Chlorella sorokiniana) prepared in M8a media with and without surfactant were measured with Ubbelohde viscometers. The Pluronic F68 surfactant was obtained from Panreac Applichem (Spain). The calibration of the viscometer was done by glycerol solutions of known viscosities. The experiments were done at room temperature, ensured with submerging the viscometers in a water bath $\left(21.5-22{ }^{\circ} \mathrm{C}\right)$. The viscosity of a solution containing $5 \mathrm{~g} \mathrm{~L}^{-1}$ Chlorella sorokiniana was applied in the model.

The surface tension of the above mentioned solutions were measured with a drop tensiometer, analysing the shape of a hanging drop (Teclis Tracker). In these experiments a microalgae concentration of $7.8 \mathrm{~g} \mathrm{~L}^{-1}$ was used.

\subsubsection{Model implementation in Matlab}

For the model simulations the reactor was divided into 400 depth and 100 height segments. The liquid fraction gradient in height was determined by a differential equation solver (ODE45), while the gas concentrations were determined by manual integration, thus the stepwise changes in height were implemented, according to Equation 5.1.

$\boldsymbol{X}(\boldsymbol{h}+\Delta \boldsymbol{h})=\boldsymbol{X}(\boldsymbol{h})+\Delta \boldsymbol{h} \cdot \boldsymbol{f}(\boldsymbol{h})$

Equation 5.1

Where $X$ indicates the variable at a certain height, $\Delta h$ is the height step, and $f$ is the function describing the differential $d x / d h$. Light and growth model results were expressed in terms of 
horizontal and vertical position and also as a function of wavelength, therefore the output was expressed in a 3D matrix. For further calculations, growth rates and production rates were summed for all wavelengths and were averaged over the liquid volume in the foam-bed. The detailed equations are presented in Appendix 5.B. Optimization of the liquid recirculation rate was done applying the Global Optimization Toolbox, GlobalSearch optimization solver. For the simulation, the following data was applied: $C_{x}=30 \mathrm{~g} \mathrm{~L}^{-1}, r_{b}=1 \mathrm{~mm}, J_{G}=0.5 \mathrm{~mm} \mathrm{~s}^{-1}, J_{w}=0.052$

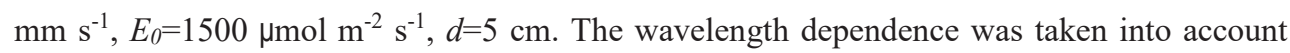
both for the absorption cross section and for the irradiance.

\subsubsection{Experimental model validation: liquid fraction of foam-beds}

In order to determine the liquid faction of Pluronic F68 foams, experiments were carried out in an automatized Foamscan (Teclis- IT Concept, Logessaigne, France). Experiments were performed at varying column height $(20.79$ and $14.15 \mathrm{~cm})$, gas flow rates $(0,2.36$ and $4.46 \mathrm{~mm}$ $\left.\mathrm{s}^{-1}\right)$, and liquid addition rates $\left(0,0.092\right.$ and $\left.0.36 \mathrm{~mm} \mathrm{~s}^{-1}\right)$. Foam was generated in a glass cylinder by blowing nitrogen gas through a metal frit with small conical holes $(30 \mu \mathrm{m}$ and $100 \mu \mathrm{m}$ hole diameter on the top and the bottom of the cone, respectively). The smaller cylinder corresponding to the lower column height has a diameter of $3.5 \mathrm{~cm}$, and $40 \mathrm{~mL}$ solution was used for foam formation, while in the bigger cylinder (diameter of $6 \mathrm{~cm}$ ) $60 \mathrm{~mL}$ solution was used. After the foam volume has reached $400 \mathrm{~cm}^{3}$, or $200 \mathrm{~cm}^{3}$ in the small column, the gas flow automatically stopped. In case of liquid addition, the foam was left for $\sim 10$ minutes to drain and thus a dry foam was achieved before the liquid addition was initiated. The evolution of the bubble sizes was monitored by image analysis at a height of $8 \mathrm{~cm}$ above the gas distributor. The bubble size was calculated by image analysis software (Foamscan). The temperature of the glass cylinder was between 24 and $30{ }^{\circ} \mathrm{C}$ in all experiments. The surfactant concentration applied was 5 or $10 \mathrm{CMC}$. The experiments were performed in duplicate. The liquid fraction of the foam was determined from conductimetric analysis of the liquid volume of the solution remaining on the bottom of the cylinder. The total liquid fraction of the foam is calculated by dividing the volume of liquid incorporated in the foam by the foam volume.

When liquid addition was applied, the average liquid fraction over the whole column was calculated by Equation 5.2.

$\varepsilon=\frac{J_{d}}{v_{\text {wetfront }}}$

Equation 5.2 
Where $J_{d}$ is the superficial liquid flow rate from the top (the liquid addition rate), and $v_{\text {wetfront }}$ is the measured liquid velocity. Thus, since the drainage only occurs via the liquid phase of the foam, not over the whole cross-sectional area, the liquid fraction can be calculated. The wet front velocity was determined by measuring the time required for the liquid added from the top to arrive to the bottom of the foaming column, and this time was divided by the height of the foam column. The moment when the wet front arrived to the bottom of the column was indicated by increase in the liquid volume, which was determined by conductivity measurements.

\subsubsection{Experimental model validation: light penetration in foam}

In order to determine the light distribution in liquid foams, fluence rate measurements were carried out at several points in depth, inside a liquid foam-bed. Fluence rate was determined by submerging a spherical light sensor (Walz, US-SQS/A) into absorbing and non-absorbing foams. Foam formation took place by distributing gas in a surfactant containing solution in a glass container of $20.5 \cdot 3 \cdot 40 \mathrm{~cm}$ (width $\mathrm{x}$ depth $\mathrm{x}$ height). The gas was distributed via a silicon tube (4 mm inner diameter, $2 \mathrm{~mm}$ wall thickness) punched with a $0.45 \mathrm{~mm}$ needle every 1.4 $\mathrm{cm}$. Foams were formed by Pluronic F68 at a concentration of $2 \mathrm{CMC}$ in a $350 \mathrm{ml}$ initial solution. The liquid solution was recirculated from the liquid layer underneath the foam to the top of the foam column by peristaltic pumps and was distributed at 4 different points at the top of the foam column. This resulted in a superficial liquid flow rate of $0.27 \mathrm{~mm} \mathrm{~s}^{-1}$. Foams were formed at a superficial gas low rate of $2.78 \mathrm{~mm} \mathrm{~s}^{-1}$, but during the measurements the gas distribution was temporarily stopped.

Fluence rate measurements were done at 0,3, 19,27 and $30 \mathrm{~mm}$ from the vertical light exposed surface, at a height of $25 \mathrm{~cm}$ and in in the middle of the reactor width $(10.25 \mathrm{~cm}$ from both sides). The light source was composed of 20 circular warm-white LED lamps (Bridgelux, BXRA W1200, Bridgelux, USA) equipped with $42 \mathrm{~mm}$ diameter Brooke-S (spot) reflector (LEDIL F'FORM OPTICS, Finland). The upper $31 \mathrm{~cm}$ of the glass tank was irradiated with $475 \mu \mathrm{mol} \mathrm{m} \mathrm{m}^{-2} \mathrm{~s}^{-1}$. A distance of $47 \mathrm{~cm}$ was kept between the reactor and the light source to ensure homogenous light distribution on the flat glass tank. Measurements were done in foams without microalgae (clear foam), and at 0.6, 2.6 and $6 \mathrm{~g} \mathrm{~L}^{-1}$ C. sorokiniana concentration. The reflected and transmitted flux from the glass tank was measured with a flat $2 \pi$ PAR quantum sensor (LI-SA-190, LI-COR, USA). The transmitted flux was measured directly behind the glass tank, while the reflected flux was measured on the light source, $45.5 \mathrm{~cm}$ from the glass 
tank, in between the LED lamps to prevent shading. The measured reflected flux was expressed relative to the reflected flux from white paper which was placed at the location of the glass tank. This represents ideal diffuse reflectance. The values were further corrected for residual ambient light by subtracting the reflected flux in case of black paper placed at the location of the glass reactor, which represents complete absorbance. The bubble size of the foam in the vessel was determined manually by image analysis, where 120 bubbles were measured.

\subsection{Results}

\subsubsection{Liquid fraction in a foam-bed photobioreactor}

The liquid fraction gradient in the foam was modelled for three cases: a) continuously rising foam-bed; b) continuously rising foam-bed with liquid addition from the top; c) rising foambed which is broken at a certain height with liquid addition from the top.

Generally, the liquid fraction of foams decreases quickly with height. The maximum liquid fraction at the bottom of the foam column is 0.26 in case of ordered foams with homogenous bubble size distribution. The model results show (Figure 5,5A) that by liquid addition, the liquid fraction of rising foam can be increased because of continuous drainage of the added water through the foam structure. When the rising foam is continuously broken, the liquid fraction is further elevated since foam breaking can be regarded as external liquid addition. By applying liquid addition or foam breaking, the liquid fraction profile is more homogenous (or 'flat') compared to the liquid fraction profile of rising foams (See Figure 5.5A, insert). This flat profile is advantageous in order to create a similar horizontal light gradient along the foam column. The simulated liquid fraction profiles correspond well to the profiles reported by P. Stevenson $[5]$.

Additional simulations showed that higher liquid addition rates $\left(J_{w}\right)$, smaller bubbles $\left(r_{b}\right)$, and higher gas flow rates $\left(J_{G}\right)$ lead to increased liquid fraction of the foam (Figure 5.5B, C, D). In order to create wet foams, liquid addition is the best way, since the gas flow rates and foam breaking in the absence of additional liquid supply have minor effect on liquid fractions compared to liquid recirculation. Decreasing bubble size also significantly increases the liquid fraction of foams. However, this would require altered gas distributer design or increased surfactant concentrations. Experiments to obtain model parameters and experiments to partly validate the model are presented in Appendix 5.A. 

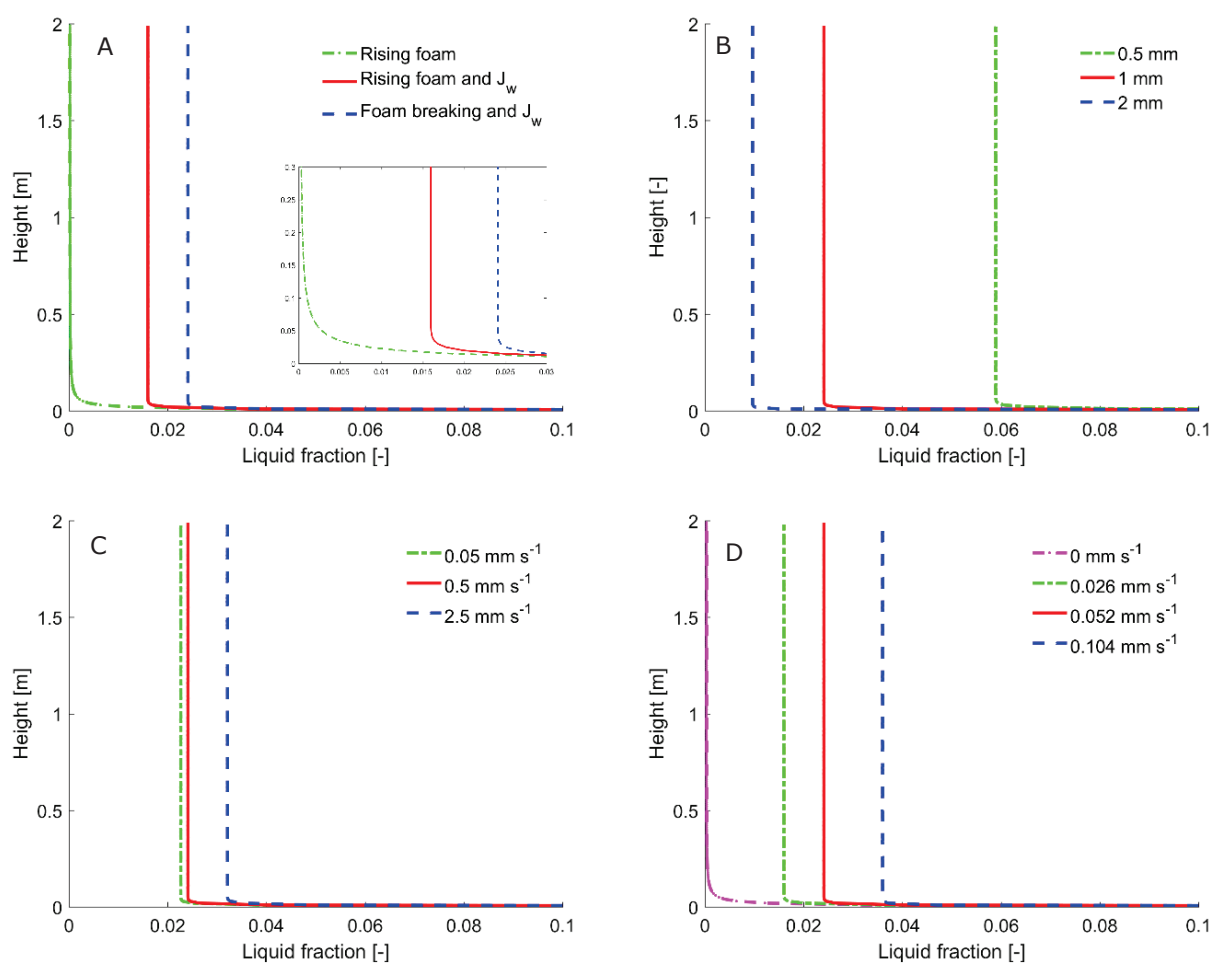

Figure 5.5. Liquid fraction of foams as a function of height in the foam-bed. (A) Liquid fraction of a rising foam, a rising foam with liquid recirculation, and a foam which is broken at the top with additional liquid recirculation. The insert is zoomed in to the same image. (B) Liquid fraction at varying bubble radius $r_{b} ;(C)$ Liquid fraction at varying superficial gas flow rate $J_{G}$; (D) Liquid fraction at varying superficial liquid recirculation rates $J_{w}$. For the simulation, the following inputs were applied: $r_{b}=1 \mathrm{~mm}, J_{G}=0.5 \mathrm{~mm} \mathrm{~s}^{-1}, J_{W}=0.052 \mathrm{~mm} \mathrm{~s}^{-1}, h=2 \mathrm{~m}$ with foam breaking at the top.

\subsubsection{Light profile in a foam-bed photobioreactor}

The fluence rate inside the photobioreactor is influenced by the biomass concentration $\left(C_{x}\right)$, bubble size $\left(r_{b}\right)$, the illuminating light intensity $\left(E_{0}\right)$ and the liquid fraction of the foam $(\varepsilon)$. The liquid fraction, as demonstrated before, is dependent again on both the superficial gas velocity $\left(J_{G}\right)$ and the superficial liquid recirculation velocity $\left(J_{w}\right)$. The fluence rate is linearly proportional to $E_{0}$ (see Appendix 5.B) and also reflectance and transmittance are linearly proportional to $E_{0}$.

On the bottom of the reactor, close to the height of foam generation the foam has a high liquid fraction, resulting in lower fluence rate compared to the top of the foam (Figure 5.6A). At the front of the reactor, the fluence rate can be higher than the incident irradiance (Figure 5.6) because of photons arriving to this layer from both forward and backward direction due to light 
scattering. This light profile is less advantageous for algae growth because of a relative increase in light saturation at the light exposed surface, and also because of at deeper points towards the back of the reactor, the light availability is decreased.

In non-absorbing foams $\left(C_{x}=0 \mathrm{~g} \mathrm{~L}^{-1}\right)$, the fluence rate peaks at approximately $1 \mathrm{~cm}$ deep in the reactor, afterwards it steeply decreases with depth (Figure 5.6B). At higher biomass concentrations, the fluence rate at the reactor front approaches the incident light intensity and decreases exponentially from the start (the peak is absent) due to both absorption and scattering (Figure 5.6B). With increasing biomass concentration, both the transmitted light and reflected light decreases due to the stronger absorption (Table 5.2).

By varying the liquid fraction of foams by varying liquid recirculation $\left(J_{w}\right)$, both scattering and absorbing behaviour of the foam changes. At low liquid fractions, light absorption is reduced due to the reduced amount of microalgae present, but also light scattering is reduced since the scattering coefficient is influenced by the liquid fraction (See Appendix 5.B). On the one hand, because of reduced light absorption the fluence rate has a higher initial value and the light gradient is less steep at lower liquid fractions (Figure 5.6C). Also the elevated reflectance and transmittance at low liquid fractions (Table 5.2) is explained by the relative decrease in absorption. On the other hand the effect of scattering is also influential and it becomes clear when equal absorption is ensured in absorbing foams with different liquid fractions, achieved by compensating for the reduced liquid fractions with elevated biomass concentrations. The case of reduced liquid fraction and increased biomass concentration enables deeper light penetration in the reactor due to a less steep light attenuation, therefore in this case a more advantageous light profile can be achieved.

Increased gas flow rates and decreased bubble sizes also result in increased liquid fraction, therefore similar light profiles are achieved as when increasing the liquid recirculation rate. Bubble radius, however, does not only influence the liquid fraction, but also affects the scattering coefficient. Larger bubble sizes reduce the extent of scattering also when the liquid fraction is kept constant (e.g. by adjusting $J_{w}$ ). Therefore, the combined effect of large bubbles on both liquid fraction and scattering result in lower fluence rates at the reactor front and a less steep decrease of fluence rate with depth compared to smaller bubbles (Fig 6D). Accordingly, reflectance is decreased and transmittance is increased compared to smaller bubbles (Table 5.2). 

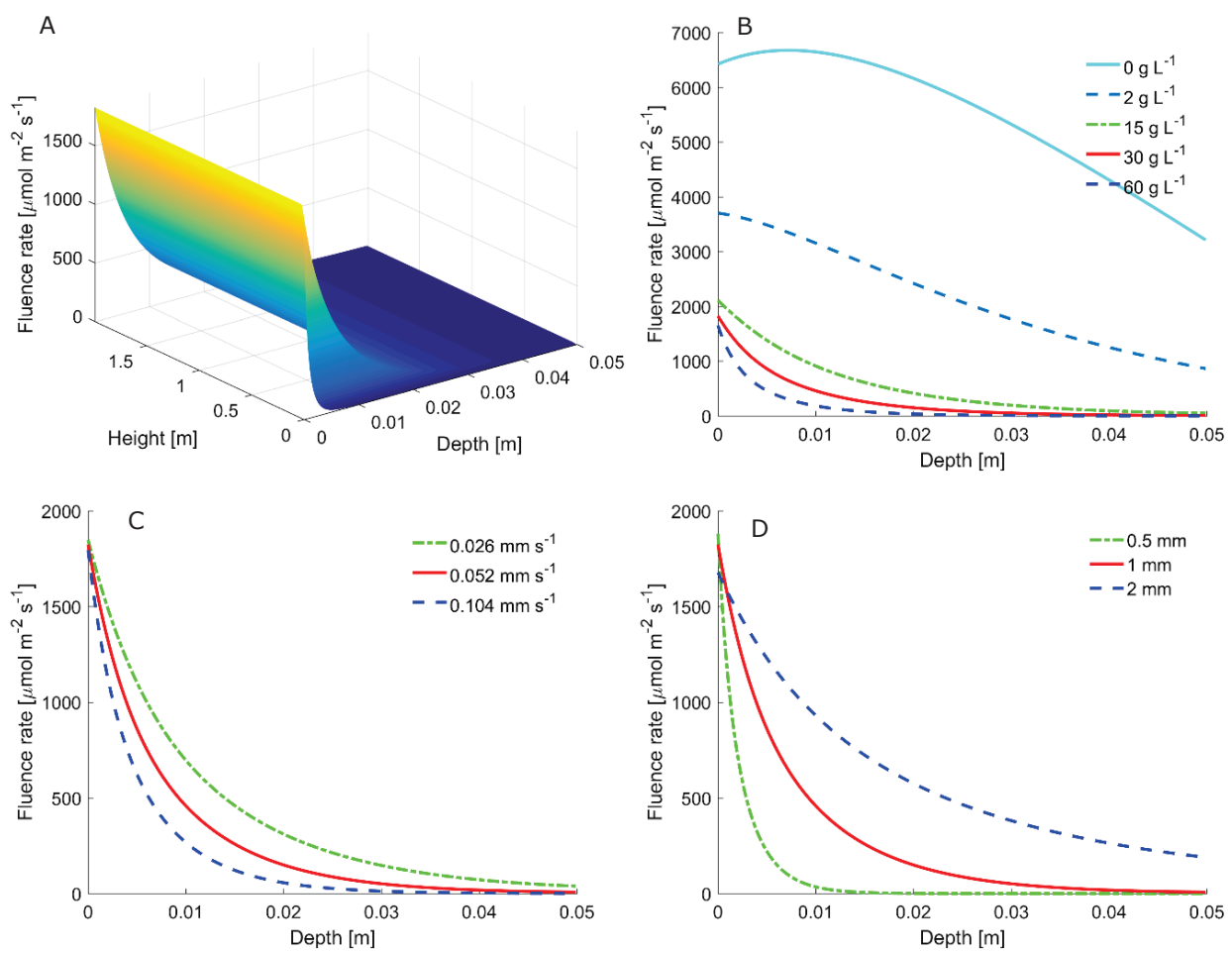

Figure 5.6. Light distribution in the reactor, (A) presented as fluence rate as a function of depth and height, $(B, C, D)$ presented as fluence rate at the mid-height of the reactor as a function of depth for $(B)$ different $C_{x}(C)$ different $J_{w}$ (D) different $r_{b}$. For the simulation, the following inputs were applied unless otherwise indicated: $r_{b}=1 \mathrm{~mm}, J_{G}=0.5$ $\mathrm{mm} \mathrm{s}^{-1}, J_{w}=0.052 \mathrm{~mm} \mathrm{~s}^{-1}, E_{0}=1500 \mu \mathrm{mol} \mathrm{m}^{-2} \mathrm{~s}^{-1}, C_{x}=30 \mathrm{~g} \mathrm{~L}^{-1}, d=5 \mathrm{~cm}, h=2 \mathrm{~m}$, assuming foam breaking at the top.

Table 5.2. Reflectance and transmittance values for a foam-bed containing varying $C_{x}$, and operating at different $J_{w}$ and $r_{b}$. For the simulation, the following default inputs were applied: $r_{b}=1 \mathrm{~mm}, J_{G}=0.5 \mathrm{~mm} \mathrm{~s}^{-1}, J_{w}=0.052 \mathrm{~mm} \mathrm{~s}^{-1}$, $E_{0}=1500 \mu \mathrm{mol} \mathrm{m}^{-2} \mathrm{~s}^{-1}, C_{x}=30 \mathrm{~g} \mathrm{~L}^{-1}, d=5 \mathrm{~cm}, h=2 \mathrm{~m}$, assuming foam breaking at the top.

\begin{tabular}{lrrrrr|rrr|rrr}
\hline & \multicolumn{4}{c|}{$C_{x}\left[\mathrm{~g} \mathrm{~L}^{-1}\right]$} & \multicolumn{3}{|c|}{$r_{b}[\mathrm{~mm}]$} & \multicolumn{3}{c}{$J_{w}\left[\mathrm{~mm} \mathrm{~s}^{-1}\right]$} \\
& 0 & 2 & 15 & 30 & 60 & 0.5 & 1 & 2 & 0.026 & 0.052 & 0.104 \\
\cline { 2 - 13 } Reflectance [\%] & 61.0 & 30.1 & 12.0 & 8.8 & 6.8 & 9.4 & 8.8 & 7.1 & 9.0 & 8.8 & 8.4 \\
Transmittance [\%] & 39.0 & 11.4 & 0.8 & 0.1 & 0.0 & 0.0 & 0.1 & 7.1 & 0.9 & 0.1 & 0.0 \\
\hline
\end{tabular}

\subsubsection{Gas transfer}

The gas transfer rate in the liquid foam-bed photobioreactor was modelled and used to calculate the gas transfer capacity and efficiency. First, the biomass production rate was calculated according to the growth model based on the local fluence rates. The biomass production rate was $53.3 \mathrm{~g} \mathrm{~L}_{\text {liquid }}{ }^{-1} \mathrm{~d}^{-1}$ under the following conditions: $C_{x}=30 \mathrm{~g} \mathrm{~L}^{-1}, d=5 \mathrm{~cm}, h=2 \mathrm{~m}, E_{0}=1500$ $\mu \mathrm{mol} \mathrm{m} \mathrm{s}^{-2}, J_{w}=0.052 \mathrm{~mm} \mathrm{~s}^{-1}, J_{G}=0.5 \mathrm{~mm} \mathrm{~s}^{-1}$. This biomass production rate determines the $\mathrm{CO}_{2}$ requirement of the growing algal culture. The gas transfer model was then used to calculate the 
carbon dioxide $\left(\mathrm{CO}_{2}\right)$ supply rate preventing $\mathrm{CO}_{2}$ limitation in the foam-bed photobioreactor. More specifically, the minimal inlet $\mathrm{CO}_{2}$ fraction was calculated when a constant superficial gas velocity was applied. It was calculated that at fixed $0.5 \mathrm{~mm} \mathrm{~s}^{-1}$ superficial gas velocity, $6.9 \%$ $\mathrm{CO}_{2}$ is required (Figure 5.7A) in order to maintain the liquid phase $\mathrm{CO}_{2}$ concentration at $5 \cdot K_{s}$ at the above mentioned conditions. The outgoing $\mathrm{CO}_{2}$ concentration in the gas phase is approaching $0.2 \%$, therefore a $\mathrm{CO}_{2}$ uptake efficiency of $97 \%$ can be obtained, and negligible $\mathrm{CO}_{2}$ is lost. As a comparison, in the most efficient conventional photobioreactors, thin-layered liquid phase photobioreactors [20], flat-panel airlift photobioreactors [21], or tubular photobioreactors [22], more than $50 \%$ of the $\mathrm{CO}_{2}$ is not taken up and lost to the environment.

The high $\mathrm{CO}_{2}$ uptake efficiency in a foam-bed photobioreactor is related to a high $\mathrm{CO}_{2}$ transfer rate. The transfer rate can be described by the product of the volumetric mass transfer coefficient $\left(k_{L} a\right)$ and the driving force for mass transfer (the difference between the partial pressure of $\mathrm{CO}_{2}$ in the gas and the liquid phases). The mean $k_{L} a$ value for $\mathrm{CO}_{2}$ in our simulations is $0.045 \mathrm{~s}^{-1}$, calculated as the multiplication of the liquid side mass transfer coefficient and the specific surface area, as explained in Appendix 5.B. Both $k_{L}$ and $a$ change over the foam column in height, but average values were calculated to be $1.54 \cdot 10^{-5} \mathrm{~m} \mathrm{~s}^{-1}$ and $2923 \mathrm{~m}^{-1}$, respectively. $k_{L} a$ values increase at reduced liquid fraction, due to the increased $a$ and $k_{L}$ values, therefore the lowest values are at the bottom of the foam-bed. The calculated $k_{L}$ value is comparable to previously reported values in foam reactors of $3 \cdot 10^{-5} \mathrm{~m} \mathrm{~s}^{-1}$, and also the $a$ value is comparable to reported values of $2150-3220 \mathrm{~m}^{-1}$ [16].

The specific surface area $a$ was calculated assuming spherical bubbles. In case of reduced liquid fractions, the calculation of $a$ therefore might be inaccurate due to bubble deformations [23], or due to unequal liquid distribution around the bubbles such as a reduced contribution of the thin liquid films [16,24]. Also a change in bubble size in height because of gas diffusion or coalescence/coarsening will lead to deviation of the specific surface area. When comparing the $k_{L}$ values of a foam-bed bioreactor with those of conventional, liquid phase photobioreactors the $k_{L}$ is lower in foam-bed reactors. This is related to reduced liquid flow and smaller gas bubbles in foam-bed photobioreactors [25]. The specific surface area $a$, on the other hand, is 60 times higher in a foam-bed photobioreactor. As a result, the $k_{L} a$ of $0.045 \mathrm{~s}^{-1}$ in the foam-bed photobioreactor is still 5 to 10 times higher compared to other systems: 0.007 or $0.009 \mathrm{~s}^{-1}$ was reported for a flat plate reactor [26,21], and $0.001-0.007 \mathrm{~s}^{-1}$ for bubble column photobioreactors [27]. 
The majority of $\mathrm{CO}_{2}$ transfer in the photobioreactor takes place at the bottom of the reactor, as illustrated in Figure 5.7. Since the gas transfer rate in foam-bed photobioreactors is high, rapid $\mathrm{CO}_{2}$ depletion takes place in the bubbles and therefore the liquid phase concentration increases quickly to the desired value $\left(5 \cdot K_{s}\right)$. This transferred $\mathrm{CO}_{2}$ is used through the whole column for the algal growth, which is related to the assumption of an ideally mixed liquid phase. The decrease in the slope of the $\mathrm{O}_{2}$ and $\mathrm{CO}_{2}$ concentration in the gas phase is due to the decreased driving force for gas transfer in height due to the increasing $\mathrm{O}_{2}$ and decreasing $\mathrm{CO}_{2}$ concentrations at higher points in the reactor. In contrast to rapid $\mathrm{CO}_{2}$ transfer, the $\mathrm{O}_{2}$ concentration increases gradually in the column because of the reduced solubility of $\mathrm{O}_{2}$, which is expressed by an increased Henry coefficient. The $\mathrm{O}_{2}$ concentration difference over the thin liquid layer surrounding the gas bubbles is therefore reduced compared to that of $\mathrm{CO}_{2}$ which therefore explains the different slopes of the driving force curve in height for the two gases (Figure 5.7B).
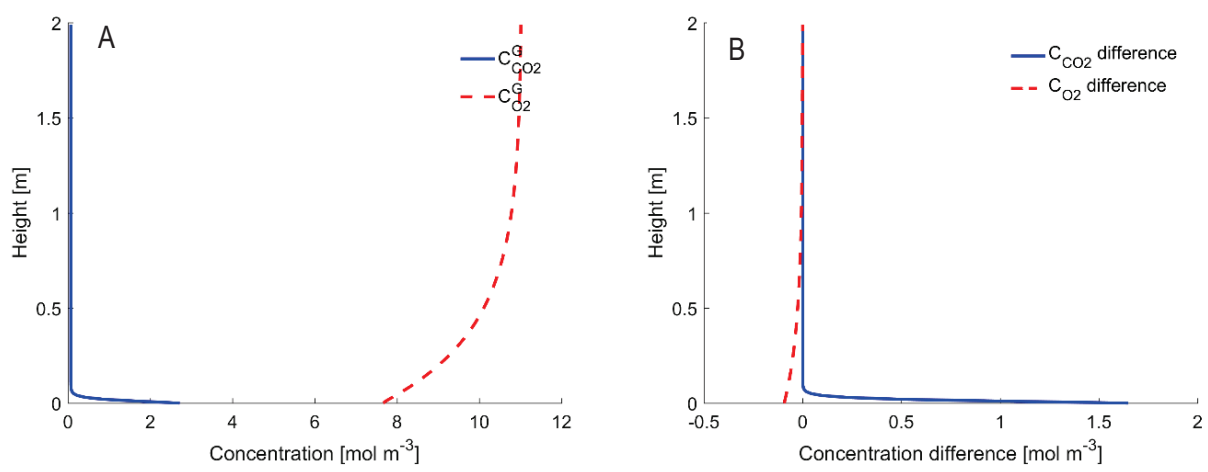

Figure 5.7. A) $\mathrm{O}_{2}$ and $\mathrm{CO}_{2}$ concentration gradient in the gas phase. B) The driving force for mass transfer expressed as the concentration difference over the stationary liquid film surrounding the gas bubbles $\left(\mathrm{C}_{i}{ }^{*}-\mathrm{C}_{i}{ }^{L}\right)$. Simulations were done at $C_{x}=30 \mathrm{~g} \mathrm{~L}^{-1}, r_{b}=1 \mathrm{~mm}, J_{G}=0.5 \mathrm{~mm} \mathrm{~s}^{-1}, E_{0}=1500 \mu \mathrm{mol} \mathrm{m}^{-2} \mathrm{~s}^{-1}, d=0.05 \mathrm{~m}, h=2 \mathrm{~m}$. To convert from mol $\mathrm{m}^{-3}$ to $\%$ of the compound in the gas phase, a factor of 2.5 can be used.

Besides the sufficient $\mathrm{CO}_{2}$ supply, also the $\mathrm{O}_{2}$ concentrations in the liquid phase is relevant for photobioreactor designs. The tolerance of microalgae are estimated to be around $120-200 \%$ air saturation [28]. However, most photobioreactors operate at severely increased $\mathrm{O}_{2}$ concentrations up to several hundred percent air saturation $[29,30]$. In the simulated liquid foam-bed photobioreactor, the liquid $\mathrm{O}_{2}$ concentration is $134 \%$ air saturation level, which is considered to be tolerable for the microalgae and therefore no inhibition effects are included. 


\subsubsection{Growth, Productivity and Energy requirement of a foam-bed photobioreactor}

Light-dependent microalgal specific growth rate can be calculated based on the local fluence rate calculated, which depends again on the biomass concentration as explained above. The productivity of the photobioreactor is the product of the biomass concentration and the average specific growth rate of the microalgae. The areal productivity of a conventional photobioreactor is thus mainly determined by the depth of the reactor and the biomass density maintained, which should be selected according to the illuminating light intensities. The light leaving the reactor should be minimal, but still allowing for the compensation for the maintenance requirements of the microalgal cells [31]. Too thin reactors in combination with low biomass densities lead to light losses, while too thick reactors result in a dark layer at the back of the reactor, where algal 'decay' takes place. Generally, in order to achieve a high biomass concentration, thin reactors are preferred [28], and there is an optimum biomass concentration where the reactor productivity is maximal [32]. In foam reactors, the liquid fraction of the foam plays an important role. Generally, foams consist of only $<10 \%$ liquid phase, and, as a result, in foambed photobioreactors biomass concentrations an order of magnitude higher than in conventional photobioreactors can be reached. This increase is not entirely proportional to the decrease in liquid fraction, since the light profile is altered due to scattering and some light is lost due to out-scattering of light from the photobioreactor as discussed before.

Besides reactor productivity, the energy invested for the production is a key factor when considering the feasibility of the system. The operational energy requirements for microalgae production in foam-bed photobioreactors depend on the energy requirements for gas supply and liquid circulations. In addition, the energy requirements for algal separation from a suspension is substantial and it scales inversely proportional to the biomass concentration.

The productivity and energy demand of a foam-bed photobioreactor is therefore highly dependent on the reactor design (dimensions of the system), light intensity, biomass concentration, and reactor operation (gas supply rate and liquid recirculation). Please note that the biomass concentration is also an operational parameter as it can be controlled by reactor dilution. Also gas bubble size in the foam has significant effect on productivity by altering the light profile. For this reason, different conditions were evaluated by model simulations: incident light intensity, gas bubble size in the foam, superficial gas velocity, biomass concentration, and reactor depth. For each condition, the areal productivity shows a peak with varying liquid recirculation rates (Figure 5.8), where the area refers to the illuminated reactor area. By 
adjusting the liquid recirculation rate for maximal productivity, the liquid fraction profile is altered to provide optimal light profiles, thus minimizing dark zones or unused light passing through the reactor. The biomass specific energy requirements are increasing with increased liquid recirculation rates, as a combined effect of increased energy for liquid recirculation and also for gas supply due to the increased hydrostatic pressure because of higher liquid fractions. Because of the dependence of the areal productivity on $J_{w}$, for further simulations at different conditions, the liquid recirculation rate was always optimized in order to achieve maximal areal biomass productivity.

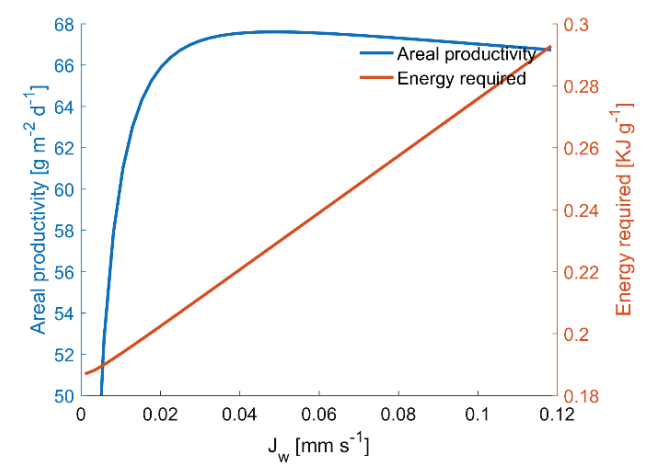

Figure 5.8. Areal productivity and biomass specific energy requirements as a function of liquid recirculation rate, $J_{w}$ under the default conditions of $C_{X}=30 \mathrm{~g} \mathrm{~L}^{-1}, r_{b}=1 \mathrm{~mm}, J_{G}=0.5 \mathrm{~mm} \mathrm{~s}^{-1}, E_{0}=1500 \mu \mathrm{mol} \mathrm{m}^{-2} \mathrm{~s}^{-1}, d=0.05 \mathrm{~m}, h=2 \mathrm{~m}$.

Increasing the reactor height does not influence the areal productivities, since the liquid fraction is constant above the first few centimetres of the reactor. The energy required for gas supply is also nearly proportional to the height (i.e. hydrostatic pressure), therefore the effect of height on reactor performance is negligible. The reactor height was arbitrarily fixed at 2 meters.

When optimising the superficial liquid recirculation rate $J_{w}$ for maximal productivity, $J_{w}$ increases from 0.01 to $4 \mathrm{~mm} \mathrm{~s}^{-1}$ when the reactor depth decreases from 10 to $1 \mathrm{~cm}$. Similarly, $J_{w}$ increases from 0.02 to $0.12 \mathrm{~mm} \mathrm{~s}^{-1}$ when the biomass concentration decreases from 60 to 15 $\mathrm{g} \mathrm{L}^{-1}$ (Table 5.3). A general trend can be observed: at higher biomass densities or in deeper reactors, the superficial liquid recirculation rate can be reduced. The resulting reduced liquid fraction allows for deeper light penetration in the reactor.

The minimal reactor depth applied, $1 \mathrm{~cm}$, resulted in the highest areal productivity and photosynthetic efficiency (biomass yield on light). These values are $74.6 \mathrm{~g} \mathrm{~m}^{-2} \mathrm{~d}^{-1}$ and $0.58 \mathrm{~g}$ $\mathrm{mol}_{\mathrm{ph}}{ }^{-1}$, respectively (Table 5.3). However, the economic feasibility of such thin reactors are 
compromised due to the high energy requirements required for the liquid recirculation to reach a liquid fraction of $20 \%$. Additionally, in thin reactors enhanced drainage is expected due to the increasing fraction of bubbles in contact with the walls, and therefore the calculated $J_{w}$ might be underestimated [33]. In deeper reactors, not only the superficial recirculation rate, but also the total volumetric recirculation flow is reduced therefore the energy requirement for liquid recirculation decreases. Also, the reduced liquid fraction in thicker reactors result in reduced power for gas supply. Therefore, deep reactors are less energy-demanding, even when expressed as energy demand per $\mathrm{g}$ of biomass produced (calculations presented in Appendix 5.B). It has to be noted that in this study we focus on energy requirements corresponding to maximal areal productivities instead of minimizing biomass specific energy requirements. Minimal energy requirements are achieved under a lower liquid recirculation rate than obtained by optimizing for maximal areal productivity.

Amongst the three different biomass densities investigated (15, 30 and $\left.60 \mathrm{~g} \mathrm{~L}^{-1}\right), 60 \mathrm{~g} \mathrm{~L}^{-1}$ resulted in the highest areal productivity of $73.2 \mathrm{~g} \mathrm{~m}^{-2} \mathrm{~d}^{-1}$ at $1500 \mu \mathrm{mol} \mathrm{m} \mathrm{m}^{-2} \mathrm{~s}^{-1}$ in a $5 \mathrm{~cm}$ deep reactor. The reduced liquid fraction required for maximal productivity at $60 \mathrm{~g} \mathrm{~L}^{-1}$ results in the reduction of scattering and therefore aids more advantageous light profiles. Besides, more absorbing media reduces the reflection as well, improving the biomass yield on light. Due to reduced liquid circulation and reduced harvest flow, the biomass specific energy requirements can be reduced from 0.49 to $0.11 \mathrm{KJ} \mathrm{g}^{-1}$ when the biomass concentration is increased from 15 to $60 \mathrm{~g} \mathrm{~L}^{-1}$.

At increased light intensities, higher liquid fractions are required in order to absorb most of the incoming light and prevent light leaving the system unused. On the one hand, the lowest incident light intensity $\left(375 \mu \mathrm{mol} \mathrm{m}^{-2} \mathrm{~s}^{-1}\right)$ results in the highest biomass yield on light, $0.88 \mathrm{~g}$ $\mathrm{C}_{\mathrm{x}} \mathrm{mol}_{\mathrm{ph}}{ }^{-1}$, because the local fluence rates are around, or below, the saturation point of photosynthesis. On the other hand, the areal productivity is lowest at the lowest light intensity. When the recirculation rates are optimized in order to reach maximal areal productivity, the energy requirements are not significantly influenced by varying light intensities. This is reasoned by that at low intensities lower liquid fraction are optimal, and therefore reduced recirculation rates $J_{w}$ have to be applied compared to higher light intensities with increased productivity.

The gas supply rate $\left(J_{G}\right)$, the liquid recirculation rate $\left(J_{w}\right)$, and the bubble size $\left(r_{b}\right)$ together influence the liquid fraction of the foam and therefore the light distribution in the foam-bed 
photobioreactor, as shown in the previous section. Elevated liquid fraction and decreasing bubble size does not only increase the absorption coefficient of the foam, but also enhances the scattering. Therefore, the light profile is altered and it becomes steeper: the fluence rate decreases more rapidly with distance from the light exposed surface. A steeper light gradient is not beneficial since there is more photosaturation in the zone close to the light exposed surface while the light limited zone becomes larger. This results in a lower biomass yield on light and a decrease in areal productivity. The energy requirement in case of increased bubble size is higher since bigger bubbles imply reduced liquid fraction, and therefore an elevated recirculation rate has to be applied as compensation. The energy required for bubble generation, however, is not taken into account. For example, more than 5 times higher recirculation rate is required when the bubble size is increased from 1 to $2 \mathrm{~mm}$, and consequently the energy requirements increase from 0.23 to $0.39 \mathrm{KJ} \mathrm{g}^{-1}$ (Table 5.3). Altered gas flow rates can also be compensated by adapting the liquid recirculation rate to maintain a constant liquid fraction and areal productivity. As such, a liquid fraction of 3\% can be maintained. Reduced gas flow rates in combination with increased recirculation rates result in the lowest energy requirement, although the influence of gas flow rate on energy requirement is minimal (Table 5.3).

Altogether, these results and additional simulations of all conditions at 15 and $60 \mathrm{~g} \mathrm{~L}^{-1}$ show that the energy requirements are minimal at high biomass concentration and deep reactors $\left(C_{x}=60 \mathrm{~g} \mathrm{~L}^{-1}, E_{0}=1500 \mu \mathrm{mol} \mathrm{m} \mathrm{s}^{-1}\right.$ and $d=10 \mathrm{~cm}$, the $\left.E=0.1 \mathrm{KJ} \mathrm{g}^{-1}\right)$. Since the highest areal productivities were achieved at $60 \mathrm{~g} \mathrm{~L}^{-1}, 1 \mathrm{~cm}$ depth and $1500 \mu \mathrm{mol} \mathrm{m}^{-2} \mathrm{~s}^{-1}$, it is possible that the optimal areal productivity is still above this biomass concentration but was not studied in our simulations because of envisioned practical limitation, including: foam instability due to too high/low liquid fractions; physical-chemical changes in the liquid properties at $C_{x}$ above 60 $\mathrm{g} \mathrm{L}^{-1}$ [34], thereby influencing foaming properties; nutrient limitations in the liquid media. Additionally, high biomass concentrations might reduce the drainage rates due to confinement of the cells and their aggregates in the foam-bed, thereby reducing liquid recirculation requirements [35].

In a bench-scale liquid foam-bed photobioreactor, an areal productivity of $51.4 \mathrm{~g} \mathrm{~m}^{-2} \mathrm{~d}^{-1}$ was achieved, with a yield on light of $0.41 \mathrm{~g} \mathrm{~mol}_{\mathrm{ph}}{ }^{-1}$ at a biomass concentration of $6.81 \mathrm{~g} \mathrm{~L}^{-1}$. These experiments were conducted with Chlorella sorokiniana in a circular foam column with a diameter of $10 \mathrm{~cm}$, illuminated with a circular light source from all directions [8]. The experimentally achieved yield on light and areal productivities are $75-80 \%$ of the predicted ones for $30 \mathrm{~g} \mathrm{~L}^{-1}$ and $5 \mathrm{~cm}$ deep rectangular reactors. This difference is explained by suboptimal $\mathrm{pH}$ 
values and other non-optimized operational parameters. For example, the experimental recirculation rate was more than 10 times higher than the one applied in the model, and also the experimental liquid dilution rate was 1.4 times higher compared to the model predictions. The experimentally obtained results therefore suggest that the model predictions are achievable when reactor operation is further optimized.

Table 5.3. Simulation results of a mathematical model of a liquid foam-bed photobioreactor. The following parameters were calculated: Superficial liquid recirculation rate $J_{w}$, areal productivity $r_{x}$ Areal, biomass specific energy requirement $E$, and liquid fraction $\varepsilon$. The following parameters were varied: depth, biomass concentration, light intensity, gas flow rate and bubble radius. For each simulation the liquid recirculation value was optimized to obtain maximal areal productivity. Simulations were done at the following default values: $C_{x}=30 \mathrm{~g} \mathrm{~L}^{-1}, r_{b}=1 \mathrm{~mm}, J_{G}=0.5 \mathrm{~mm}$ $\mathrm{s}^{-1}, E_{0}=1500 \mu \mathrm{mol} \mathrm{m} \mathrm{m}^{-2} \mathrm{~s}^{-1}, d=0.05 \mathrm{~m}, h=2 \mathrm{~m}$.

\begin{tabular}{|c|c|c|c|c|c|c|}
\hline & & $\begin{array}{c}J_{w} \\
{\left[\mathrm{~mm} \mathrm{~s}^{-1}\right]}\end{array}$ & $\begin{array}{c}r_{x}^{\text {Areal }} \\
{\left[\mathrm{g} \mathrm{m}^{-2} \mathrm{~d}^{-1}\right]}\end{array}$ & $\begin{array}{c}Y_{x / p h} \\
{\left[\mathrm{~g} \mathrm{~mol}_{p h}{ }^{-1}\right]}\end{array}$ & $\begin{array}{c}E \\
{\left[\mathrm{KJ} \mathrm{g}^{-1}\right]}\end{array}$ & $\begin{array}{c}\mathcal{\varepsilon} \\
{[-]}\end{array}$ \\
\hline \multirow{3}{*}{$\begin{array}{l}\text { Depth } \\
d[\mathrm{~cm}]\end{array}$} & 1 & 4.00 & 74.6 & 0.58 & 0.79 & 0.20 \\
\hline & 5 & 0.05 & 67.6 & 0.52 & 0.23 & 0.03 \\
\hline & 10 & 0.01 & 65.9 & 0.51 & 0.21 & 0.01 \\
\hline \multirow{3}{*}{$\begin{array}{l}\text { Biomass conc. } \\
\quad C_{x}\left[\mathrm{~g} \mathrm{~L}^{-1}\right]\end{array}$} & 15 & 0.12 & 60.3 & 0.47 & 0.49 & 0.04 \\
\hline & 30 & 0.05 & 67.6 & 0.52 & 0.23 & 0.03 \\
\hline & 60 & 0.02 & 73.1 & 0.56 & 0.11 & 0.01 \\
\hline \multirow{3}{*}{ 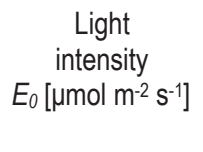 } & 375 & 0.02 & 28.6 & 0.88 & 0.23 & 0.02 \\
\hline & 750 & 0.03 & 46.5 & 0.72 & 0.23 & 0.02 \\
\hline & 1500 & 0.05 & 67.6 & 0.52 & 0.23 & 0.03 \\
\hline \multirow{3}{*}{$\begin{array}{c}\text { Superficial gas } \\
\text { velocity } \\
J_{G}\left[\mathrm{~mm} \mathrm{~s}^{-1}\right]\end{array}$} & 0.05 & 0.05 & 67.6 & 0.52 & 0.23 & 0.03 \\
\hline & 0.5 & 0.05 & 67.6 & 0.52 & 0.23 & 0.03 \\
\hline & 2.5 & 0.03 & 67.6 & 0.52 & 0.24 & 0.03 \\
\hline \multirow{3}{*}{$\begin{array}{l}\text { Bubble radius } \\
\quad r_{b}[\mathrm{~mm}]\end{array}$} & 0.5 & 0.01 & 57.4 & 0.44 & 0.19 & 0.02 \\
\hline & 1 & 0.05 & 67.6 & 0.52 & 0.23 & 0.03 \\
\hline & 2 & 0.27 & 74.5 & 0.57 & 0.39 & 0.03 \\
\hline
\end{tabular}




\subsubsection{Foam-bed photobioreactors versus traditional photobioreactors}

In order to relate the performance of the foam-bed photobioreactor to traditional photobioreactors, their performance is simulated under the previously selected conditions: $C_{x}=30 \mathrm{~g} \mathrm{~L}^{-1}, d=5 \mathrm{~cm}, h=2 \mathrm{~m}, E_{0}=1500 \mu \mathrm{mol} \mathrm{m}^{-2} \mathrm{~s}^{-1}, J_{w}=0.052 \mathrm{~mm} \mathrm{~s}^{-1}, J_{G}=0.5 \mathrm{~mm} \mathrm{~s}^{-1}$. The total biomass specific energy requirement for gas supply is $0.23 \mathrm{KJ} \mathrm{g}^{-1}$, which consists of $8 \mathrm{~J} \mathrm{~g}^{-1}$ for gas supply, $43 \mathrm{~J} \mathrm{~g}^{-1}$ for liquid recirculation and $180 \mathrm{~J} \mathrm{~g}^{-1}$ for separation by a centrifuge.

The average growth rate $\mu^{a v}$ in our simulations is $0.07 \mathrm{~h}^{-1}$. A biomass volumetric productivity $r_{x}$ of $53.3 \mathrm{~g} \mathrm{~L}_{\text {liquid }}{ }^{-1} \mathrm{~d}^{-1}$ is achieved, which is significantly higher than values achieved in other short light path photobioreactors [36,37]. However, this increase is due to the low liquid content of the foam, and therefore the volumetric productivities calculated for the whole reactor are reduced by a factor equivalent to the liquid fraction of the foam. For a more fair comparison, the productivity per illuminated surface area was therefore calculated. An areal productivity of $67.7 \mathrm{~g} \mathrm{~m}^{2} \mathrm{~d}^{-1}$ is predicted for the foam-bed photobioreactor, which is lower than areal productivities achieved in $14 \mathrm{~mm}$ light path reactors with C. sorokiniana under similar conditions. These productivities are 184.8 and $119 \mathrm{~g} \mathrm{~m}^{-2} \mathrm{~d}^{-1}$ under 2100 and $1500 \mu \mathrm{mol} \mathrm{m} \mathrm{m}^{-2} \mathrm{~s}^{-1}$, respectively [36,37]. Since the reactor depth has a significant influence on areal productivity, flat panels and foam-bed reactors of the same depth can be compared. At a depth of $1 \mathrm{~cm}$, the areal productivity of the foam-bed at a biomass concentration of $60 \mathrm{~g} \mathrm{~L}^{-1}$ is $3.06 \mathrm{~g} \mathrm{~m}^{2} \mathrm{~h}^{-1}$, approaching the areal productivity reported for flat panels, $3.89 \mathrm{~g} \mathrm{~m}^{2} \mathrm{~h}^{-1}$ at $9.7 \mathrm{~g} \mathrm{~L}^{-1}$ biomass concentration [38].

The reason behind the lower areal productivities in a foam-bed photobioreactor is the reduced photosynthetic efficiency. Our simulations predicted $0.52 \mathrm{~g}$ biomass per mol of photons, which is lower compared to the ones achieved in flat panels, which range between 0.8 and $1 \mathrm{~g} \mathrm{~mol}_{\mathrm{ph}^{-1}}$ $[36,37,39]$. The lower photosynthetic efficiency in the foam-bed is due to light loss by reflection ( $9 \%$ of the incident light) and also due to a steeper and thus more unfavourable light gradient.

The energy requirement of microalgae production in a flat plate photobioreactor and in a foambed photobioreactor can be compared when the same microalgal species are used and the same light intensities applied. The operational energy requirement of flat panel photobioreactors is only determined by the gas supply. For our simulation, the energy requirement of a large-scale flat panel photobioreactor described by Ruiz et al. [2] was considered with the dimensions of $0.5 \mathrm{~m}$ height and $2 \mathrm{~cm}$ depth. In that study, a gassing rate of $0.32 \mathrm{vvm}$ is described, translating to $0.0027 \mathrm{~m} \mathrm{~s}^{-1}$ superficial velocity. The biomass specific energy requirement was then 
calculated considering maximal productivities realized at lab-scale, $119 \mathrm{~g} \mathrm{~m}^{-2} \mathrm{~d}^{-1}$ achieved at $1500 \mu \mathrm{mol} \mathrm{m}^{-2} \mathrm{~s}^{-1}$ in a $14 \mathrm{~mm}$ light path reactor [37], in order to define an advantageous scenario for flat panels under continuous illumination. Finally, a biomass concentration of $2.3 \mathrm{~g} \mathrm{~L}^{-1}$ was assumed as described by Ruiz et al. [2] for $2 \mathrm{~cm}$ deep flat panels located in Curacao or Saudi Arabia. This biomass concentration is required in order to estimate the energy requirement for algae harvesting. According to these inputs, the biomass specific energy requirement for gas supply to flat panels is $0.38 \mathrm{KJ} \mathrm{g}^{-1}$ while the separation requires $2.35 \mathrm{KJ} \mathrm{g}^{-1}$, resulting in a total biomass specific energy requirement of $2.73 \mathrm{KJ} \mathrm{g}^{-1}$.

Foam-bed photobioreactors result in a decreased energy requirements for the gas supply. Reduction in energy takes place since the hydrostatic pressure exerted by the foam-bed is reduced by more than one order of magnitude compared to an equivalent water column and therefore less gas compression is required. In addition, the volumetric gas flow is reduced in foam-beds. Foam-bed photobioreactors require reduced superficial gas velocities compared to flat panels, since the gas transfer capacity is much higher due to the high interfacial area. In flat panel photobioreactors the $k_{L} a$ is highly dependent on $J_{G}$, high $J_{G}$ values are required to achieve sufficient gas transfer [26]. However, in foam reactors applying liquid recirculation, an additional energy requirement for pumping liquid to the top of the reactor has to be considered. The energy requirement for the liquid recirculation is linearly related to the height of the photobioreactor and the liquid recirculation rate. The liquid pumps for the liquid recirculation require 5.3 times more energy compared to the gas supply to the foam-bed photobioreactor, therefore liquid recirculation is the major production-related operational energy requirement. Still, the total operational energy requirement of algal production in foam-bed photobioreactors are reduced compared to flat panels. For foam-beds, a power input to a reactor unit is $0.08 \mathrm{~W}$, while for flat panels, $0.26 \mathrm{~W}$ is required.

The energy required for biomass separation is reduced in foam-bed photobioreactor compared to flat panels. The separation energy requirements are inversely related to the biomass concentration, therefore, an increase from 2.3 to $30 \mathrm{~g} \mathrm{~L}^{-1}$ results therefore in a proportional energy saving on separation. Thus, the energy required for biomass separation in foam-bed photobioreactors is $7.7 \%$ of that in flat panels. Since harvesting is more energy-demanding process compared to gas or liquid supply, (5.4 $\mathrm{KJ} \mathrm{L}^{-1}$ of algal suspension) the biomass concentration is critical for in the total energy requirement of the complete microalgae production process. Overall, the total production and harvesting energy requirements in the foam-bed photobioreactor are only $8.5 \%$ of flat panels $\left(0.23 \mathrm{vs} .2 .73 \mathrm{~kJ} \mathrm{~g}^{-1}\right)$. 


\subsection{Conclusions}

A mathematical model was developed to evaluate the potential of liquid foam-bed photobioreactors. The model allowed simulation of the liquid fraction gradient, light penetration, microalgal growth, and gas transfer in foams under different conditions. Our simulations for the liquid fraction and the light profile were in a good agreement with experimental data. Model parametric sensitivity was studied for bubble radius, gas flow rate, liquid recirculation rate, light intensity, reactor depth, and biomass density. The model provided insight to the effect of the above listed parameters on areal productivity and energy requirements.

Our predictions at $30 \mathrm{~g} \mathrm{~L}^{-1}$ biomass and $1500 \mu \mathrm{mol} \mathrm{m}^{-2} \mathrm{~s}^{-1}$ resulted in a biomass yield on light of $0.52 \mathrm{~g} \mathrm{~mol}_{\mathrm{ph}}{ }^{-1}$ and an areal productivity of $67.7 \mathrm{~g} \mathrm{~m}^{-2} \mathrm{~d}^{-1}$, while $0.23 \mathrm{KJ}$ energy is required for the production of 1 gram dry biomass. Although yield on light and areal productivity are lower than those in optimized flat panel photobioreactors, the energy demand is significantly lower in foam-bed photobioreactors. The lower biomass yield on light is related to light scattering in foams and thereby increasing light reflection and also resulting in a less advantageous light profile in foams. However, when practice allows, and cultivation at $60 \mathrm{~g} \mathrm{~L}^{-}$ ${ }^{1}$ turns out to be feasible, these differences will be minimized. Moreover, the biomass specific energy requirement for microalgae production in foam-bed reactors is calculated to be only $8.5 \%$ of those in flat panels. These reduced energy requirements are due to a) increased biomass densities reducing the harvesting energy requirements; b) decreased gas supply due to increased gas transfer in foams ( $\sim 6$ times increased $\left.k_{L} a\right)$ and decreased pressure of the foam column. In addition, the $\mathrm{CO}_{2}$ uptake efficiency is severely improved and almost all $\mathrm{CO}_{2}$ is taken up by the microalgal culture (97\%). In conclusion, liquid foam-bed is a promising technology for microalgae cultivation. 


\section{Appendix 5.A: Parameter determination and model validation}

\section{A.1 Parameter determination}

According to our experimental data, 10 CMC Pluronic F68 increased the viscosity of demineralised water with $11 \%$, while the addition of microalgae (Chlorella sorokiniana) at a concentration of $5 \mathrm{~g} \mathrm{~L}^{-1}$ further increased the viscosity of the surfactant solution by $10 \%$ (total of $21 \%$ increase compared to demineralised water). The results are presented in Table 5.A.1.

Table 5.A.1. Dynamic viscosity of surfactant solution and inorganic media enriched with surfactant and microalgae.

\begin{tabular}{lc}
\hline Solution & Dynamic viscosity [mN s m-2] \\
\hline Demineralised water (value obtained from literature [40]) & 0.969 \\
10 CMC Pluronic F68 solution & 1.0763 \\
10 CMC Pluronic F68 $+5 \mathrm{~g} \mathrm{~L}^{-1}$ microalgae solution & 1.1755 \\
\hline
\end{tabular}

The surface tension of M8a media, MilliQ water, microalgae solution, Pluronic F68 solution and the combination of surfactant and microalgae solution were measured, and the results are presented in Table 5.A.2. The surface tension of a 10 CMC Pluronic F68 solution with microalgae $\left(7.8 \mathrm{~g} \mathrm{~L}^{-1}\right)$ is $45 \mathrm{mN} \mathrm{m}^{-1}$.

Table 5.A.2. Surface tension of MilliQ water and inorganic media enriched with surfactant and/or microalgae.

\begin{tabular}{lc}
\hline Solution & Surface tension $\left[\mathrm{mN} \mathrm{m}^{-1}\right]$ \\
\hline MilliQ water & 73.62 \\
M8a media & 72.33 \\
$7.8 \mathrm{~g} \mathrm{~L}-1$ microalgae solution & 63.06 \\
10 CMC Pluronic F68 & 49.21 \\
10 CMC Pluronic F68 $+7.8 \mathrm{~g} \mathrm{~L}^{-1}$ microalgae solution & 44.95 \\
\hline
\end{tabular}

Since the both viscosity and surface tension measurements were done at a $21.5^{\circ} \mathrm{C}$, thus a lower temperature than $37{ }^{\circ} \mathrm{C}$ used in the model, a factor for temperature correction was introduced based on the relative change in viscosity/surface tension of water between those temperatures [40], and those calculated values were applied in the model. 


\section{A.2 Validation of liquid fraction model}

In order to validate the liquid fraction model, the average liquid fraction of the foam was experimentally determined and compared to the model outputs. The experimental conditions, including the measured bubble radius and liquid fraction values are presented in Table 5.A.3. The increased bubble radius under conditions $\mathrm{C}$ and $\mathrm{D}$ compared to $\mathrm{A}$ and $\mathrm{B}$ are due to the elapsed time and consequent coalescence before the liquid addition is started, in order to create 'dry' foam.

When applying the measured $r_{b}$ for the model simulations, the predicted liquid fraction is significantly higher than the experimental one. Comparable predictions to the experimental values can be achieved by applying increased bubble sizes for the model. This correction is reasoned since its well-known that bubble size measurements by image analysis of bubbles taken at the reactor wall are underestimating the real bubble sizes [41,42]. Also, the bubble sizes were measured at a certain height, and the further increase of the bubble size in height due to e.g. coarsening were not measured. This assumption is further supported by the observation of Yazhgur et. al. [14], that the drainage model applied predicts the liquid fraction most accurate when instead of the mean radius, the radius corresponding to big bubbles are used, since big bubbles determine the foam permeability and govern the foam drainage. This remains true even if those big bubbles are rare.

With a factor of 2.3 to correct for the bubble size, the deviation in predicting the liquid fraction is always smaller than $10 \%$ for all different experimental conditions A-D (Table 5.A.3). This correction factor was determined by minimizing the sum of the squares of the differences in liquid fraction between model and experiments. In the model simulations presented in the Manuscript and Appendix 5.B, a fixed and homogenous bubble size of $1 \mathrm{~mm}$ was applied. These idealized model simulations are therefore not affected. When scaling up the foam-bed photobioreactor, however, preferably a foam-bed is created with homogenous bubbles size and/or the model predictions are improved by taking into account accurate bubble size distribution measurements. 
Table 5.A.3. Liquid fractions according to measurements $\left(\varepsilon^{m}\right)$ and predictions $\left(\varepsilon^{\text {Prediction }}\right)$ and the experimental conditions during the validation of the liquid fraction model. For each experimental condition, the listed variables which serve as inputs for the model predictions are height $(h)$, superficial gas flow velocity $\left(J_{G}\right)$, measured bubble radius $r_{b}{ }^{m}$ and superficial liquid addition rate $\left(J_{w}\right)$. In the simulations for condition $A$ and $B$, the liquid flux was calculated according to the theory of Yazhgur [14], since a rising foam was modelled, while for conditions $C$ and $D$ a standing foam with liquid addition was modelled, therefore the total liquid flux in the foam equals the extra liquid addition rate.

\begin{tabular}{|c|c|c|c|c|c|c|c|c|}
\hline \multirow[b]{2}{*}{ Condition } & \multicolumn{2}{|c|}{ Liquid fraction } & \multicolumn{6}{|c|}{ Experimental conditions } \\
\hline & $\begin{array}{c}\varepsilon^{m} \\
{[\%]}\end{array}$ & $\begin{array}{c}\mathcal{\varepsilon}^{\text {Prediction }} \\
{[\%]}\end{array}$ & $\begin{array}{c}h \\
{[\mathrm{~cm}]}\end{array}$ & $\begin{array}{c}J_{G} \\
{\left[\mathrm{~mm} \mathrm{~s}^{-1}\right]}\end{array}$ & $\begin{array}{c}r_{b}^{m} \\
{[\mathrm{~mm}]}\end{array}$ & $\begin{array}{c}J_{w} \\
{\left[\mathrm{~mm} \mathrm{~s}^{-1}\right]}\end{array}$ & $\begin{array}{c}\text { Surfactant } \\
\text { concentration }\end{array}$ & $\begin{array}{c}J_{f} \\
{\left[\mathrm{~mm} \mathrm{~s}^{-1}\right]}\end{array}$ \\
\hline$A$ & 11.06 & 10.75 & 20.79 & 3.46 & 0.168 & 0.00 & $10 \mathrm{CMC}$ & calculated \\
\hline B & 8.09 & 8.79 & 14.15 & 2.36 & 0.168 & 0.00 & $5 \mathrm{CMC}$ & calculated \\
\hline C & 3.83 & 4.2 & 20.79 & 0.00 & 0.378 & 0.092 & $10 \mathrm{CMC}$ & $J_{f}=-J_{W}$ \\
\hline D & 8.01 & 8.22 & 20.79 & 0.99 & 0.378 & 0.36 & $10 \mathrm{CMC}$ & $J_{f}=-J_{w}$ \\
\hline
\end{tabular}




\section{A.3 Validation of light model}

The light model was validated by measuring the fluence rate at several positions inside the foam-bed. In addition, the reflectance and transmittance was measured from the glass vessel filled with foam. Since the spherical light sensor (Walz US-SQS/A) measures fluence rate accurately only between $-150^{\circ}$ and $+150^{\circ}$ incident angles because of a handlebar fixed on the sphere, a correction factor was introduced to recalculate the real fluence rate in a complete isotropic light field from the measured value. This factor was calculated to be 1.3 based on the angular distribution of the fluence rate measured by the sensor which was provided by Walz (Walz Gmbh, Germany).

The fluence rate inside the foam is plotted in Figure 5.A.1 for 5 different depths with $475 \mu \mathrm{mol}$ $\mathrm{m}^{-2} \mathrm{~s}^{-1}$ incident irradiance. In most cases, the corrected fluence rate is closer to the model predictions, indicating that the assumption of isotropic light is not incorrect. Predicted fluence rates and reflectance/transmittance values show some deviations from the experimental values in the different cases with different biomass concentrations.

The prediction of fluence rate in non-absorbing, clear foam is slightly higher compared to the measured and corrected value. This might be due to light escaping also at the sides of the glass container, therefore reducing the experimental fluence rate. The sum of the measured transmittance and reflectance in non-absorbing foams are over $100 \%$, possibly due to the inaccuracy in reflectance measurements. An error in the light reflection measurements is more likely since the measurement point was further from the glass vessel, and therefore light spreading took place. This was corrected by the fraction of light that arrives to the measurement point when a white and black paper sheet was applied instead of the foam vessel. The white surface is assumed to represent complete diffuse reflectance while the black surface represents complete absorbance. Therefore, these measurements depend on many parameters, such as the absorption and reflection from the paper sheets and the angular distributions of reflections from both the foam and the white paper. As expected, for the model simulations the sum of reflection and transmission is $100 \%$ and the light balance closes. Considering the measurement error in the reflectance measurements, the model predicts the light distribution well.

In foam with increasing microalgae concentration, the simulations predict a steeper decrease of fluence rate with depth compared to the measurements (Figures 5.A.1 B, C and D). The reflectance and transmittance values are systematically underestimated by the model compared to the measured values. It is unsure whether the deviations are due to the inaccuracy in the 
measurements, since the light balance does not close, or are related to an incomplete model description. In the model, the absorption cross section of a photoacclimated microalgal mass culture was applied, which does not necessarily represent the absorption cross section of the culture used in the experiments. Also, inaccuracies in bubble size measurements or inhomogeneous liquid supply from the top might result in an altered liquid fraction, leading to different reflectance/transmittance.

The light model outputs, such as, reflectance, transmittance and fluence rate profile in depth show the same pattern as the measured values. These patterns include the reflectance and transmittance decrease with increasing biomass concentration and also the increase of local fluence rate at the front of the reactor (up to 5 times the irradiance) and its consequent decrease in depth due to absorption and scattering. Therefore, in terms of the most important characteristics of light transport, good predictions are obtained. 

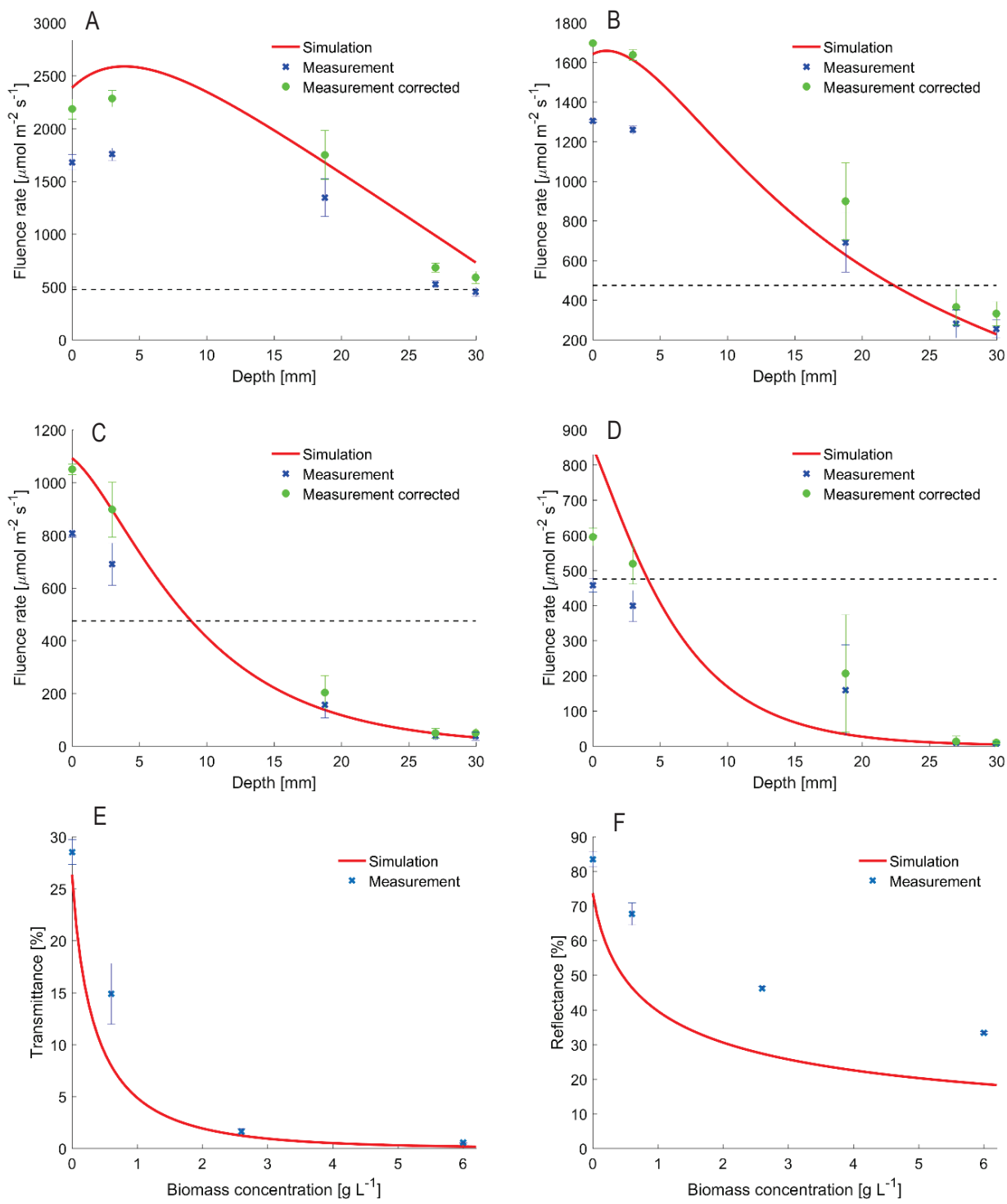

Figure 5.A.1. Light validation experiments and corresponding model simulations: A) Clear foam; B) $0.6 \mathrm{~g} \mathrm{~L}^{-1}$ microalgae concertation C) $2.6 \mathrm{~g} \mathrm{~L}^{-1}$ microalgae concentration D) $6 \mathrm{~g} \mathrm{~L}^{-1}$ microalgae concentration. The horizontal dashed line indicates the incident light intensity. E) represents the transmittance and F) the reflectance from the glass container. The error bars represent the standard deviation of three or more measurements. 


\section{Appendix 5.B: Model description}

\section{B.1 Model parameters, inputs and variables}

The values of the fixed model parameters are listed in Table 5.B.1. While most parameters were obtained from literature, some were calculated in this study noted as 'calculated' in the last column, and others were experimentally determined noted as 'measured'. Model inputs which could be varied in the simulations are listed in Table 5.B.2, and all remaining variables within the model structure are listed in Table 5.B.3. Subscripts and superscripts, in addition to the ones in the previous tables, are collected in Table 5.B.4.

Table 5.B.1. List of model parameters.

\begin{tabular}{|c|c|c|c|c|}
\hline \multicolumn{5}{|c|}{ Parameters } \\
\hline Symbol & Description & Value & Units & Ref. \\
\hline$\rho$ & Liquid phase density & 993.21 & $\mathrm{~kg} \mathrm{~m}^{-3}$ & $\mathrm{a}[40]$ \\
\hline$\mu_{L}$ & Liquid phase dynamic viscosity & $8.4110^{-4}$ & $\mathrm{~kg} \mathrm{~m}^{-1} \mathrm{~s}^{-1}(\mathrm{~Pa} \mathrm{~s})$ & $\begin{array}{l}\text { Measured, } \\
\text { corrected } \mathrm{b}[40]\end{array}$ \\
\hline$\sigma$ & Liquid phase equilibrium surface tension & 0.0436 & $\mathrm{Nm}^{-1}\left(\mathrm{~kg} \mathrm{~s}^{-2}\right)$ & $\begin{array}{l}\text { Measured, } \\
\text { corrected } \mathrm{c}[43]\end{array}$ \\
\hline$g$ & Gravitational acceleration & 9.81 & $\mathrm{~m} \mathrm{~s}^{-2}$ & \\
\hline $\boldsymbol{\varepsilon}_{\max }$ & $\begin{array}{l}\text { Maximum liquid fraction of ordered } \\
\text { foams } \\
\text { (foams with monodisperse } r_{b} \text { ) }\end{array}$ & 0.26 & - & {$[14]$} \\
\hline$n_{1}-n_{4}$ & $\begin{array}{l}\text { Parameters for the permeability of foam } \\
\text { for mobile interfaces (dependent on the } \\
\text { interfacial mobility/rigidity of the } \\
\text { interfaces; mobile interfaces are used) }\end{array}$ & $\begin{array}{l}1.5,425,2.7 \\
2.2\end{array}$ & - & {$[14]$} \\
\hline$a$ & $\begin{array}{l}\text { Parameter for the osmotic pressure } \\
\text { expression for ordered foams, } \\
\text { representing polydispersity of } r_{b}\end{array}$ & 7.3 & - & [14] \\
\hline$R$ & Ideal gas constant & 0.08206 & $\mathrm{~L} \mathrm{~atm} \mathrm{~K} \mathrm{~K}^{-1} \mathrm{~mol}^{-1}$ & \\
\hline$M D$ & $\begin{array}{l}\text { Molar density of } \mathrm{CO}_{2}, \mathrm{O}_{2} \text { and } \mathrm{N}_{2}(1 \mathrm{~atm} \text {, } \\
37^{\circ} \mathrm{C} \text { ) }\end{array}$ & 39.2913 & $\mathrm{~mol} \mathrm{~m}^{-3}$ & Calculated \\
\hline $\mathrm{H}_{\mathrm{CO} 2}$ & Henry's constant for $\mathrm{CO}_{2}, 37^{\circ} \mathrm{C}$ & 0.0408 & atm $\mathrm{mol}^{-1} \mathrm{~m}^{3}$ & [44] \\
\hline $\mathrm{H}_{02}$ & Henry's constant for $\mathrm{O}_{2}, 37^{\circ} \mathrm{C}$ & 0.9223 & atm $\mathrm{mol}^{-1} \mathrm{~m}^{3}$ & [44] \\
\hline$\bigoplus_{\mathrm{CO} 2}$ & Diffusion coefficient $\mathrm{CO}_{2}$ in water $37^{\circ} \mathrm{C}$ & $2.504610^{-9}$ & $\mathrm{~m}^{2} \mathrm{~s}^{-1}$ & [45] \\
\hline$\bigoplus_{02}$ & Diffusion coefficient $\mathrm{O}_{2}$ in water, $37^{\circ} \mathrm{C}$ & $2.624410^{-9}$ & $\mathrm{~m}^{2} \mathrm{~s}^{-1}$ & [46] \\
\hline$P_{T}$ & Atmospheric pressure & 1 & atm & \\
\hline$M W_{x}$ & $\begin{array}{l}\text { Molar weight microalgal biomass } \\
\text { expressed in C-moles (Chlorella) }\end{array}$ & 22.26 & $\mathrm{~g} \mathrm{~mol}^{-1}$ & {$[47]$} \\
\hline$a_{x}(\lambda)$ & $\begin{array}{l}\text { Wavelength-specific light absorption } \\
\text { coefficient (Chlorella) }\end{array}$ & $\begin{array}{l}\text { Supplemen- } \\
\text { tary material }\end{array}$ & $\mathrm{m}^{2} \mathrm{~mol}^{-1}$ & $d[37]$ \\
\hline
\end{tabular}

Table continued on next page 
Table 5.B.1. continued

\begin{tabular}{|c|c|c|c|c|}
\hline Symbol & Description & Value & Units & Ref. \\
\hline$Y_{X / s}$ & Yield biomass on sugar (Chlorella) & 0.59 & $\mathrm{~mol}_{\mathrm{x}} \mathrm{mol}_{\mathrm{s}}^{-1}$ & $\begin{array}{l}\text { Using } \mathrm{NH}_{4} \text { or } \\
\text { urea [10] }\end{array}$ \\
\hline$m_{s}$ & Maintenance parameter (Chlorella) & $2.510^{-6}$ & $\mathrm{~mol}_{\mathrm{s}} \mathrm{mol}_{\mathrm{x}}^{-1} \mathrm{~s}^{-1}$ & {$[10]$} \\
\hline$Y_{s / p h}{ }^{\text {MAX }}$ & Maximum yield sugar on light (Chlorella) & 0.1 & $\mathrm{~mol}_{\mathrm{s}} \mathrm{mo}_{\mathrm{Iph}}{ }^{-1}$ & [10] \\
\hline$q_{s}^{\text {MAX }}$ & $\begin{array}{l}\text { Maximum production of sugar } \\
\text { (Chlorella) }\end{array}$ & $1.310^{-4}$ & $\mathrm{~mol}_{\mathrm{s}} \mathrm{mol}_{x}^{-1} \mathrm{~s}^{-1}$ & For $\mathrm{NH}_{4}[10]$ \\
\hline$Y_{c 02 / x}$ & Yield of $\mathrm{CO}_{2}$ on biomass (Chlorella) & -0.94 & $\mathrm{~mol}_{\mathrm{co} 2} \mathrm{~mol}_{x}^{-1}$ & $\begin{array}{l}\text { Calculated on } \\
\text { urea }^{\mathrm{e}}\end{array}$ \\
\hline$Y_{02 x}$ & Yield of $\mathrm{O}_{2}$ on biomass (Chlorella) & 1.18975 & $\mathrm{molo}_{2} \mathrm{mox}^{-1}$ & $\begin{array}{l}\text { Calculated on } \\
\text { urea }{ }^{\text {e }}\end{array}$ \\
\hline$n_{g}$ & Refractive index of glass & 1.5 & - & [48] \\
\hline$n_{w}$ & Refractive index of water & 1.33 & - & [48] \\
\hline$n_{a}$ & Refractive index of air & 1 & - & [48] \\
\hline$r_{21}$ & Reflectivity of surfaces for diffuse light & 0.4913 & - & Calculated \\
\hline$r_{c e}$ & Reflectivity for collimated light (front) & 0.04332 & - & Calculated \\
\hline$r_{c e}{ }^{\prime}$ & Reflectivity for collimated light (back) & 0.04332 & - & Calculated \\
\hline$E_{n}(\lambda)$ & Spectral distribution of sunlight & $\begin{array}{l}\text { Supplemen- } \\
\text { tary material }\end{array}$ & $\mathrm{nm}^{-1}$ & ASTM G173-03 \\
\hline$g_{s}$ & Anisotropy of scattering & 0 & - & \\
\hline$K_{s}$ & Monod half-rate constant, $\mathrm{CO}_{2}$ conc. & $8.610^{-3}$ & $\mathrm{~mol} \mathrm{~m}^{-3}$ & [49] \\
\hline \multirow[t]{3}{*}{ Air } & Volume fraction $\mathrm{CO}_{2}$ & 0.0004 & - & [50] \\
\hline & Volume fraction $\mathrm{O}_{2}$ & 0.20946 & & \\
\hline & Volume fraction $\mathrm{N}_{2}$ & 0.78084 & & \\
\hline$\eta_{\text {Gpump }}$ & Efficiency of gas pump & 75 & $\%$ & [2] \\
\hline$\eta_{\text {Lpump }}$ & Efficiency of liquid pump & 70 & $\%$ & [18] \\
\hline$E^{C x, \text { sep }}$ & $\begin{array}{l}\text { Energy required per } \mathrm{m}^{3} \text { processed } \\
\text { suspension }\end{array}$ & $5.410^{6}$ & $\mathrm{~J} \mathrm{~m}^{-3}$ & [19] \\
\hline
\end{tabular}

a For the liquid phase density the value of water at $37^{\circ} \mathrm{C}$ is used and the effect of microalgae and salts is neglected. ${ }^{b}$ Since the viscosity measurements were done at a $21.5^{\circ} \mathrm{C}$, thus a lower temperature than $37^{\circ} \mathrm{C}$ used in the model, a factor for temperature correction was introduced based on the relative decrease of viscosity of water between those temperatures [40].

c For the surface tension measurements a temperature correction factor was introduced according to the relative surface tension change of water between 20 and $35^{\circ} \mathrm{C}$ [43].

d The absorption cross section spectra of a photoacclimated microalgal mass culture of Chlorella sorokiniana were used [37].

e The elemental composition and molar mass of Chlorella was determined by Duboc et al. [47]. However, since phosphorus is not included in that elemental composition, the suggestion from Blanken et al. [10] was applied: 0.01 moles of phosphorus per carbon-mole of biomass $\left(\mathrm{mol}_{\mathrm{x}}\right)$. Therefore, the elemental composition of Chlorella was thus assumed to be $\mathrm{CH}_{1.78} \mathrm{O}_{0.36} \mathrm{~N}_{0.12} \mathrm{P}_{0.01}$. According to the stoichiometry of the growth of Chlorella, considering urea as nitrogen source, and phosphate as phosphor source, $Y_{\mathrm{CO}_{2} / \mathrm{x}}$ and $Y_{\mathrm{O} 2 / \mathrm{x}}$ can be calculated.

${ }^{\mathrm{f}}$ The sunlight emission spectra was obtained from NREL, the Reference Solar Spectral Irradiance at Air Mass 1.5 was used (http://rredc.nrel.gov/solar/spectra/am1.5/). Conversion of irradiance $\left(\mathrm{W} \mathrm{m}^{-2}\right)$ to photon flux density ( $\mu \mathrm{mol}$ $\mathrm{m}^{-2} \mathrm{~s}^{-1}$ ) was done according to Planck's Law. 
Table 5.B.2. List of model inputs.

\begin{tabular}{|c|c|c|c|c|}
\hline \multicolumn{5}{|c|}{ Inputs for the model } \\
\hline Symbol & Meaning & Value(s) & Unit & Comment/ref \\
\hline$c_{x}$ & Concentration of biomass in the liquid phase & variable & $\mathrm{Cmol} \mathrm{m}^{-3}$ & $\mathrm{~g} \mathrm{~L}^{-1}$ conversion [36] \\
\hline$h \times d \times w$ & Reactor dimensions (height $x$ depth $\mathrm{x}$ width) & variable & $\mathrm{m}$ & \\
\hline$J_{G}$ & Gas superficial velocity & variable & $\mathrm{m} \mathrm{s}^{-1}$ & \\
\hline$r_{b}$ & Radius of gas bubble & variable & $\mathrm{m}$ & \\
\hline$J_{w}$ & Superficial liquid recirculation rate & variable & $\mathrm{m} \mathrm{s}^{-1}$ & \\
\hline$E_{0}$ & Irradiance & variable & $\mu \mathrm{mol} \mathrm{m} \mathrm{m}^{-2} \mathrm{~s}^{-1}$ & \\
\hline $\mathrm{C}_{\mathrm{CO}^{2}}{ }^{\mathrm{Lin}}$ & Inlet liquid $\mathrm{CO}_{2}$ concentration & 0.043 & $\mathrm{~mol} \mathrm{~m}^{-3}$ & $5 \cdot K_{s}^{a}$ \\
\hline$C_{02}^{L, i n}$ & Inlet liquid $\mathrm{O}_{2}$ concentration & 0.2271 & $\mathrm{~mol} \mathrm{~m}^{-3}$ & Air-equilibriumb \\
\hline
\end{tabular}

a In order to achieve sufficient $\mathrm{CO}_{2}$ supply in the inlet flow, $\mathrm{HCO}_{3}$ - is added in a concentration that at $\mathrm{pH} 7$ the liquid phase contains $5 \cdot K_{s}$ dissolved $\mathrm{CO}_{2}$ concentration.

${ }^{b}$ Since the liquid inlet is in equilibrium with air, its $\mathrm{O}_{2}$ concentration can be calculated from Henry's law at $37^{\circ} \mathrm{C}$.

Table 5.B.3. List of variables within the model. Height, depth and wavelength dependencies are not listed in this table, in the text these are indicated by brackets after the variable.

\begin{tabular}{|c|c|c|}
\hline \multicolumn{3}{|c|}{ Variables during simulations } \\
\hline Symbol & Meaning & Unit \\
\hline$V^{R}$ & Total volume of the reactor, $V^{R}=h \cdot d \cdot w$ & $\mathrm{~m}^{3}$ \\
\hline$V_{L}$ & Liquid volume in the reactor, $V_{L}=V^{R} \cdot \varepsilon^{a v}$ & $\mathrm{~m}^{3}$ \\
\hline$A^{\text {light }}$ & Illuminated surface area of the reactor, $A^{\text {light }=h \cdot w}$ & $\mathrm{~m}^{2}$ \\
\hline$F^{G}$ & Volumetric gas flow rate, $F^{G}=J_{G} \cdot W \cdot d$ & $\mathrm{~m}^{3} \mathrm{~s}^{-1}$ \\
\hline$F^{R}$ & Recirculation flow rate, $F R=J_{w} w \cdot d$ & $\mathrm{~m}^{3} \mathrm{~s}^{-1}$ \\
\hline$\varepsilon$ & Volumetric liquid fraction in the foam & - \\
\hline$y$ & Vertical position in reactor (height coordinates),values from 0 to $h$ & $\mathrm{~m}$ \\
\hline$z$ & Horizontal position in reactor (depth coordinates), values from 0 to $d$ & $\mathrm{~m}$ \\
\hline$F^{L}$ & Inflow and outflow rate for continuous operation & $\mathrm{m}^{3} \mathrm{~s}^{-1}$ \\
\hline$\varepsilon^{\infty}$ & Liquid fraction far away from the bottom in steady state & - \\
\hline$\Pi$ & Osmotic or disjoining pressure of the foam & $\mathrm{N} \mathrm{m}^{-2}$ \\
\hline$k(\varepsilon)$ & Foam permeability normalized by the square of bubble radius & - \\
\hline$J_{f}$ & Superficial net liquid velocity in the foam column & $\mathrm{m} \mathrm{s}^{-1}$ \\
\hline$J_{d}$ & Superficial liquid drainage rate (appears only in validation, Appendix 5.A) & $\mathrm{m} \mathrm{s}^{-1}$ \\
\hline$\lambda$ & Wavelength, values from 400 to $700 \mathrm{~nm}$ & $\mathrm{~nm}$ \\
\hline$r$ & Reflection factor from different interfaces & - \\
\hline$R, R_{\text {flux }}$ & Reflectivity, Reflected flux & $\%, \mu \mathrm{mol} \mathrm{m}{ }^{-2} \mathrm{~s}^{-1}$ \\
\hline$T, T_{\text {flux }}$ & Transmittance, Transmitted flux & $\%, \mu \mathrm{mol} \mathrm{m}{ }^{-2} \mathrm{~s}^{-1}$ \\
\hline$\mu_{a}$ & Absorption coefficient & $m^{-1}$ \\
\hline$I_{a}$ & Absorption length & $\mathrm{m}$ \\
\hline$l_{a}^{\text {soln }}$ & Absorption length of liquid solution & $\mathrm{m}$ \\
\hline$\mu_{s}$ & Scattering coefficient & $m^{-1}$ \\
\hline$I^{*}$ & Optical mean free path & $\mathrm{m}$ \\
\hline$\mu_{t}$ & Total attenuation coefficient; $\mu_{t}=\mu_{a}+\mu_{s}$ & $m^{-1}$ \\
\hline$\mu_{\text {eff }}$ & Effective attenuation coefficient; $\mu_{e f f}=\sqrt{3 \cdot \mu_{a} \cdot\left(\mu_{a}+\mu_{s} \cdot(1-g)\right)}$ & $m^{-1}$ \\
\hline
\end{tabular}

Table continued on next page 
Table 5.B.3. Continued

\begin{tabular}{|c|c|c|}
\hline Symbol & Meaning & Unit \\
\hline$F_{+}$ & $\begin{array}{l}\text { Hemispherical photon flux per unit area }(d A)-\text { integration over } 2 \pi \text { solid } \\
\text { angle in forward direction (same as incident light) }\end{array}$ & $\mu \mathrm{mol} \mathrm{m} \mathrm{m}^{-2} \mathrm{~s}^{-1}$ \\
\hline$F$ & $\begin{array}{l}\text { Hemispherical photon flux per unit area }(d A) \text { - integration over } 2 \pi \text { solid } \\
\text { angle in backward direction (opposite as incident light) }\end{array}$ & $\mu \mathrm{mol} \mathrm{m} \mathrm{m}^{-2} \mathrm{~s}^{-1}$ \\
\hline$\Phi_{t}$ & Fluence rate, total photon flux density & $\mu \mathrm{mol} \mathrm{m} \mathrm{m}^{-2} \mathrm{~s}^{-1}$ \\
\hline$S_{+} / S_{-}$ & Coefficients applied in the light model & $m^{-2}$ \\
\hline$A_{+} / A_{-} / q /$ & Coefficients applied in the light model & - \\
\hline \multicolumn{3}{|l|}{$A_{+-} / A_{-+}$} \\
\hline$r_{x}$ & Biomass volumetric productivity & $\mathrm{mol}_{\mathrm{x}} \mathrm{m}^{-3} \mathrm{~s}^{-1}\left(\mathrm{~g} \mathrm{~L}^{-1} \mathrm{~d}^{-1}\right)$ \\
\hline$r_{x}^{\text {Areal }}$ & Biomass areal productivity & $\mathrm{g} \mathrm{h}^{-1} \mathrm{~m}^{-2}$ \\
\hline \multirow[t]{2}{*}{$q_{i}$} & Specific production rate of compound $\mathrm{i}$ & $\mathrm{mol}_{\mathrm{i}} \mathrm{mol}_{x}^{-1} \mathrm{~s}^{-1}$ \\
\hline & Consumption rates own a negative value & \\
\hline$\mu$ & Biomass specific growth rate & $\mathrm{s}^{-1}$ \\
\hline$r_{i}$ & $\begin{array}{l}\text { Volumetric production rate of compound } \mathrm{i} \text { in the liquid phase; } \\
r_{i}=q_{i} \cdot c_{x} \text {. Consumption rates own a negative value }\end{array}$ & moli $m^{-3} \mathrm{~s}^{-1}$ \\
\hline$Y_{x / p h}$ & Biomass yield on photons & $\begin{array}{l}\mathrm{mol}_{x} \mathrm{molph}^{-1}\left(\mathrm{~g} \mathrm{molph}^{-}\right. \\
\text {1) }\end{array}$ \\
\hline$V_{\text {slip }}$ & $\begin{array}{l}\text { Slip velocity (velocity difference between the gas and the liquid phases } \\
\text { in rising foam) }\end{array}$ & $\mathrm{m} \mathrm{s}^{-1}$ \\
\hline$k_{L, I}$ & Liquid-side mass transfer coefficient for compound i & $\mathrm{m} \mathrm{s}^{-1}$ \\
\hline a & Specific surface area of the foam & $m^{-2}$ \\
\hline$k_{L} a_{i}$ & Volumetric mass transfer coefficient for compound i & $\mathrm{s}^{-1}$ \\
\hline$C_{i}$ & Concentration of compound $i$ & $\mathrm{~mol} \mathrm{~m}^{-3}$ \\
\hline$C_{i}{ }^{*}$ & Interface equilibrium concentration of compound i & $\mathrm{mol} \mathrm{m}^{-3}$ \\
\hline$P_{i}$ & Partial pressure of compound i & Atm \\
\hline$y_{i}$ & Molar fraction of compound i & - \\
\hline$\eta_{\mathrm{CO} 2}$ & $\mathrm{CO}_{2}$ uptake efficiency & - \\
\hline$P$ & Power & W \\
\hline$p_{F}$ & Hydrostatic pressure of the foam & $\mathrm{Pa}$ \\
\hline$E$ & Energy requirements per gram of biomass produced & $\mathrm{kJ} \mathrm{g}_{\mathrm{x}}-1$ \\
\hline$E^{S e p}$ & Energy requirement for the algal separation per gram of biomass & $\mathrm{kJ} \mathrm{g}_{\mathrm{x}}-1$ \\
\hline
\end{tabular}

Table 5.B.4. Subscripts and superscripts of the model variables and inputs.

\begin{tabular}{|c|c|c|c|}
\hline \multicolumn{2}{|c|}{ Superscript } & \multicolumn{2}{|c|}{ Subscript } \\
\hline$L$ & Liquid phase & $x$ & Biomass \\
\hline$F$ & Foam phase & $\mathrm{CO}_{2}$ & Carbon dioxide \\
\hline G & Gas phase & $\mathrm{O}_{2}$ & Oxygen \\
\hline av & Averaged for the liquid phase & $s$ & Sugar \\
\hline w & In case of liquid addition & ph & Photon \\
\hline$f b$ & In case of foam-breaking & $a / g / w$ & Air/glass/ water \\
\hline stst & Steady-state & & \\
\hline avPAR & Averaged for the PAR region & & \\
\hline $\ln /$ out & Inlet/ outlet & & \\
\hline * & Interface & & \\
\hline
\end{tabular}




\section{B.2 Liquid fraction model}

The net superficial liquid velocity $\left(J_{f}\right)$ in a rising foam is obtained from the sum of upward liquid movements and the relative downward liquid movements (Eq. 5.B.1). The first term of Eq. 5.B.1 represents the liquid flow upward in the rising foam due to the gas supply. The second term gives the slip velocity or drainage rate, which is the difference between the flow velocities of the liquid and gas phases. The superficial slip velocity can be calculated by Darcy's law. This slip velocity is characterized by the foam properties (bubble size, permeability and viscosity), gravity, and the osmotic pressure gradient in the foam in height [14]. Osmotic pressure is determined by the surface tension, bubble size, liquid fraction, maximal liquid fraction of the foam near the interface and constants related to the dispersity of bubble size distribution (Eq. 5.B.2). Foam permeability is related to foam structure (liquid fraction) and the physical-chemical properties of foams related to the mobility of interfaces (Eq. 5.B.3). The net liquid superficial velocity, the osmotic pressure in the foam and the permeability are defined in Eq. 5.B.1-5.B.3 [14].

$J_{f}=\frac{\varepsilon \cdot J_{G}}{1-\varepsilon}+\frac{r_{b}^{2} \cdot k(\varepsilon)}{\mu_{L}}\left(\frac{d \Pi(\varepsilon)}{d y}-\rho \cdot g\right)$ Equation 5.B.1

$\Pi=\frac{\alpha \cdot \sigma \cdot\left(\varepsilon_{\max }-\varepsilon\right)^{2}}{r_{b} \cdot \sqrt{\varepsilon}}$ Equation 5.B.2

$k(\varepsilon)=\frac{\varepsilon^{n_{1}}}{n_{2} \cdot\left(1-n_{3} \cdot \varepsilon+n_{4} \cdot \varepsilon^{2}\right)^{2}}$

In this study, a monodisperse foam with mobile interfaces was considered. The assumptions of mobile interfaces for Pluronic F68 solutions are based on literature evaluation of physicochemical foam properties [14]. $n_{1}-n_{4}$ are semi-empirical parameters, which values are $1.5,425$, 2.7, 2.2 [14,51], for mobile interfaces, while $\alpha=7.3$ and $\boldsymbol{\varepsilon}_{\max }=0.26$ for ordered, monodisperse foams [14]. For the bubble size, a Sauter mean bubble radius is used by Yazhgur et al. [14], which matches with the radius of bubbles under the assumption of uniform bubble size.

In order to obtain the liquid fraction gradient in height, $\frac{d \varepsilon}{d y}$, the gradient of $\frac{d \Pi}{d y}$ should be expressed in terms of $\frac{d \varepsilon}{d h}$, resulting in the following expression (Eq. 5.B.4).

$\frac{d \Pi}{d y}=\frac{\alpha \cdot \sigma \cdot\left(\varepsilon-\varepsilon_{\max }\right) \cdot\left(3 \varepsilon+\varepsilon_{\max }\right)}{2 \cdot r_{b} \cdot \varepsilon^{\frac{3}{2}}} \cdot \frac{d \varepsilon}{d y}$

Equation 5.B.4 
This term can be substituted to the expression for $J_{f}$ (Eq. 5.B.1), and by re-arranging the equation to give $\frac{d \varepsilon}{d y}$, the liquid fraction profile can be predicted (Eq. 5.B.8). However, firstly the net liquid superficial velocity in the foam, $J_{f}$ has to be determined.

The net superficial liquid velocity in the foam, $J_{f}^{\text {stst }}$ is constant throughout a rising foam in steady-state due to conservation of mass. This steady state is defined such that the liquid fraction is constant in time at any position in the foam-bed (but the liquid fraction can still differ between different positions). Since the net superficial liquid velocity is constant, its value far away from the bottom, where the liquid fraction gradient can be neglected $\left(\frac{d \varepsilon}{d y}=0\right.$ and therefore $\left.\frac{d \Pi}{d y}=0\right)$ is representative for the whole column. This condition can be used in order to obtain $J_{f}^{s t s t}$. To express this, firstly the liquid fraction far away from the bottom $\left(\varepsilon^{\infty}\right)$ has to be expressed, which is obtained from Eq. 5.B.1 by applying $\frac{d J_{f}}{d \varepsilon}=0$ (Eq. 5.B.5 and 5.B.6).

$\frac{J_{G}}{\left(\varepsilon^{\infty}-1\right)^{2}}-\frac{\rho \cdot g \cdot r_{b}{ }^{2}}{\mu_{L}} \cdot \frac{d k\left(\varepsilon^{\infty}\right)}{d \varepsilon^{\infty}}=0$

Equation 5.B.5

Where $\frac{d k\left(\varepsilon^{\infty}\right)}{d \varepsilon^{\infty}}=\frac{\varepsilon^{\infty\left(n_{1-1}\right)} \cdot\left[\left(n_{1}-4\right) \cdot n_{4} \cdot \varepsilon^{\infty}+\left(2-n_{1}\right) \cdot n_{3} \cdot \varepsilon^{\infty}+n_{1}\right]}{n_{2} \cdot\left(n_{4} \cdot \varepsilon^{\infty}-n_{3} \cdot \varepsilon^{\infty}+1\right)^{3}}$

Equation 5.B.6

From $\varepsilon^{\infty}$, the steady state liquid superficial velocity, $J_{f}^{\text {stst }}$ throughout the foam column can be obtained. Substituting $\varepsilon^{\infty}$ to Eq. 5.B.1 and neglecting the osmotic pressure term result in the following expression for the steady state superficial liquid velocity:

$J_{f}^{s t s t}=\frac{\varepsilon^{\infty} \cdot J_{G}}{1-\varepsilon^{\infty}}-\frac{\rho \cdot g \cdot r_{b}^{2} \cdot \varepsilon^{\infty n_{1}}}{\mu \cdot n_{2} \cdot\left(1-n_{3} \cdot \varepsilon^{\infty}+n_{3} \cdot \varepsilon^{\infty}\right)^{2}}$

Equation 5.B.7

Finally, the liquid fraction gradient in height can be expressed by applying $J_{f}^{\text {stst }}$ (Eq. 5.B.8).

$\frac{d \varepsilon}{d y}=\left[\left(J_{f}^{s t s t}-\frac{\varepsilon \cdot J_{G}}{1-\varepsilon}\right) \frac{\mu \cdot n_{2} \cdot\left(1-n_{3} \cdot \varepsilon+n_{4} \cdot \varepsilon^{2}\right)^{2}}{r_{b}{ }^{2} \cdot \varepsilon^{n_{1}}}+\rho \cdot g\right] \frac{2 \cdot r_{b} \varepsilon^{\frac{3}{2}}}{\alpha \cdot \sigma \cdot\left(\varepsilon-\varepsilon_{\max }\right) \cdot\left(3 \cdot \varepsilon+\varepsilon_{\max }\right)} \quad$ Equation 5.B.8

This first order differential equation (Eq. 5.B.8) integrated with $\varepsilon_{\max }$ as the boundary condition for $y=0$ provides the vertical liquid fraction gradient of foam in steady-state.

The liquid recirculation in photobioreactors can be modelled as external liquid addition from the top to a rising foam column. This will increase the liquid fraction throughout the foam due to enhanced liquid drainage via the foam structure. The liquid addition reduces the net liquid superficial velocity, which eventually will have a downward orientation, also in case of a rising foam column. According to theory from Stevenson et al. [5], the liquid addition rate, $J_{w}$ can be 
accommodated to Eq. 5.B.8 by subtracting $J_{w}$ from the above calculated $J_{f}^{\text {stst }}$ (Eq. 5.B.9). Thus, the 'new' steady-state net liquid superficial velocity in case of liquid addition, $J_{f}^{w, s t s t}$ can be described as $J_{f}^{w, s t s t}=J_{f}^{s t s t}-J_{w}$, where $J_{f}^{s t s t}$ represents the liquid superficial velocity of a rising foam, without liquid addition, as calculate by Eq. 5.B.8. In case the foam is broken at a certain height, the liquid liberated from the broken bubbles can be seen as additional liquid supply $\left(J_{w}{ }^{f b}\right)$. In this case the net superficial liquid velocity will be 0 , since the additional flux due to bubble collapse exactly matches the liquid flux that reaches the foam breaker. Therefore, the net flux without foam breaking $\left(J_{f}^{s t s t}\right)$ equals to the additional flux resulting from foam breaking $\left(J_{w}^{f b}\right)$, $J_{w}^{f b, s t s t}=J_{f}^{s t s t}{ }_{-} J_{w}^{f b}=0$. In case of both foam breaking and liquid addition, $J_{w}$ includes both of these fluxes, thus $J_{f}^{w+f b, s t s t}=-J_{w}$.

$\frac{d \varepsilon}{d h}=\left[\left(J_{f}^{s t s t}-J_{w}-\frac{\varepsilon \cdot J_{G}}{1-\varepsilon}\right) \cdot \frac{\mu \cdot n_{2}\left(1-n_{3} \cdot \varepsilon+n_{4} \cdot \varepsilon^{2}\right)^{2}}{r_{b}{ }^{2} \cdot \varepsilon^{n_{1}}}+\rho \cdot g\right] \cdot \frac{2 \cdot r_{b} \cdot \frac{3}{2}}{\alpha \cdot \sigma \cdot\left(\varepsilon-\varepsilon_{\max }\right) \cdot\left(3 \cdot \varepsilon+\varepsilon_{\max }\right)}$

Equation 5.B.9

\section{B.3 Light model}

Light transport in the foam was modelled according to the light diffusion theory. The concept from Welch et al. served as a baseline for the calculations considering an infinite wide and high slab with finite thickness [15].

The total fluence rate at a given position is the sum of the scattered and the non-scattered fluence rates (Eq. 5.B.10). The scattered fluence rate can be described by the first part of Eq. 5.B.10, where $F_{+}(z)$ and $F_{-}(z)$ are hemispherical fluxes which are defined as energy flux through unit area in either forward or backward direction $\left(F_{+}(z)\right.$ and $F_{-}(z)$, respectively). The second part of the equation represents the direct fluence rate. The fluence rate and the hemispherical fluxes in our calculations are expressed in $\mu \mathrm{mol} \mathrm{m} \mathrm{m}^{-2} \mathrm{~s}^{-1}$.

$\Phi t(y, z, \lambda)=2 \cdot\left[F_{+}(y, z, \lambda)+,F_{-}(y, z, \lambda)\right]+\left(1-r_{c e}\right) \cdot E_{0} \cdot \exp \left(-\mu_{t}(y, \lambda) \cdot z\right)$

Equation 5.B.10

In Eq. 5.B.10, the internally reflected collimated light from the glass wall at the back of the reactor was neglected, since collimated light attenuates rapidly due to both scattering and absorption. Therefore, collimated light arriving to the back of the reactor is low especially in case of high biomass densities, which, when multiplied with the reflection factor $r_{c e}$, in the order of a few percentages, results in negligible light intensities. 
The hemispherical fluxes can be described at depth ' $z$ ' by the following equations (Eq. 5.B.11 and 5.B.12). These expressions are also height dependent as the optical properties of the foam are influenced by the height-dependent liquid fraction. In addition, some optical properties depend on the wavelength, such as specific light absorption.

$$
\begin{gathered}
F_{+}(z, y, \lambda)=\left(1-r_{c e}\right) \cdot E_{0} \cdot\left[q(y, \lambda) \cdot A_{-+}(y, \lambda) \cdot \exp \left(\mu_{e f f}(y, \lambda) \cdot z\right)+\right. \\
\left.A_{+-}(y, \lambda) \cdot \exp \left(-\mu_{e f f}(y, \lambda) \cdot z\right)+A_{+} \cdot \exp \left(-\mu_{t}(y, \lambda) \cdot z\right)\right] \\
F_{-}(z, y, \lambda)=\left(1-r_{c e}\right) \cdot E_{0} \cdot\left[A_{-+}(y, \lambda) \cdot \exp \left(\mu_{e f f}(y, \lambda) \cdot z\right)+\right. \\
\left.q(y, \lambda) \cdot A_{+-}(y, \lambda) \cdot \exp \left(-\mu_{e f f}(y, \lambda) \cdot z\right)+A_{-} \cdot \exp \left(-\mu_{t}(y, \lambda) \cdot z\right)\right]
\end{gathered}
$$

The variables $q(y, \lambda), A_{+-}(y, \lambda), A_{-+}(y, \lambda), \mu_{\text {eff }}(y, \lambda), \mu_{t}(y, \lambda), S+(y, \lambda), A_{-}(y, \lambda), A_{+}(y, \lambda)$ are only a function of the scattering and absorption coefficients, the anisotropy of scattering $g_{S}$, and the reflection factors for collimated and diffuse light, $r_{c e}$ and $r_{2 I}$ respectively.

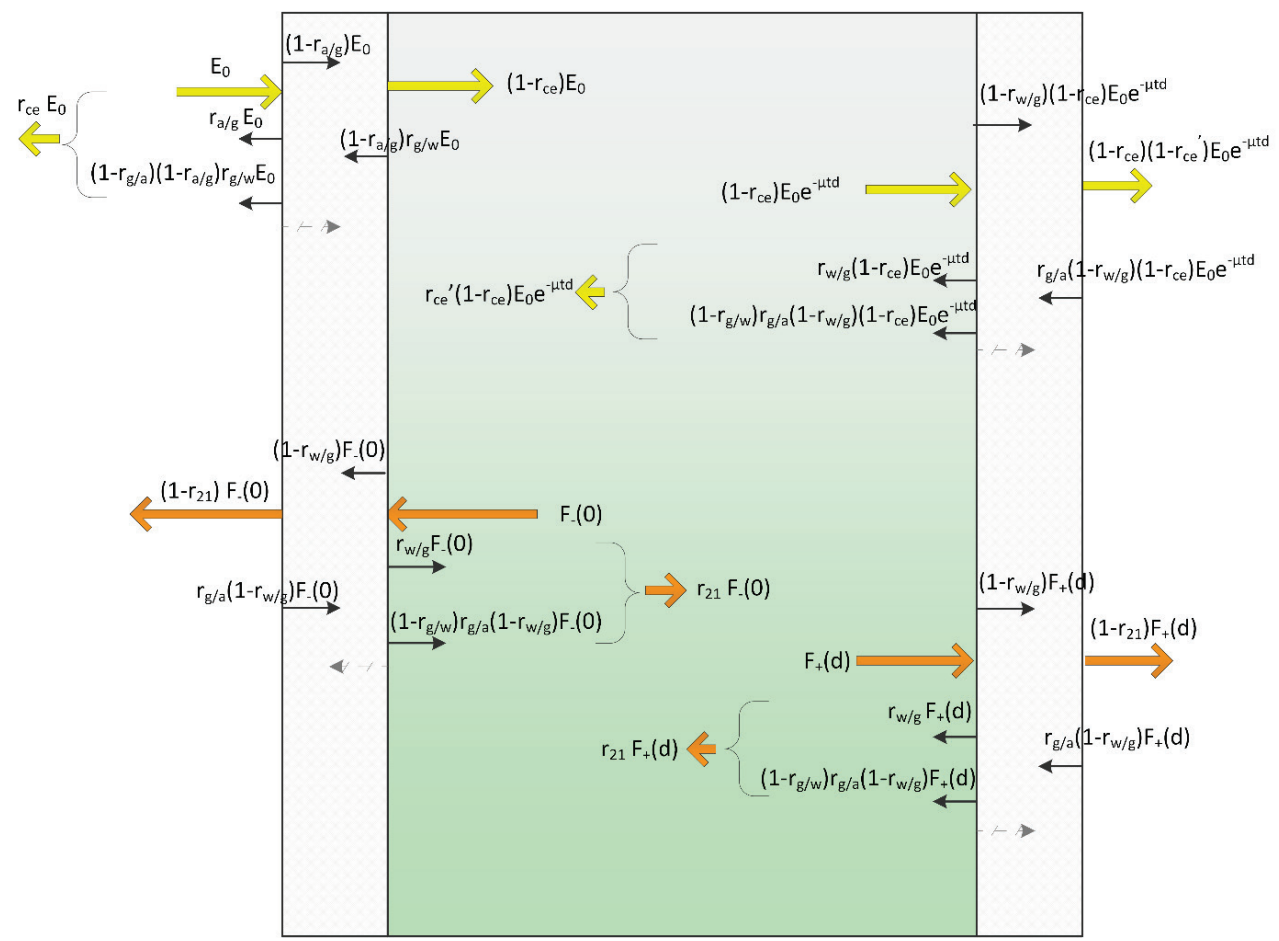

Figure 5.B.1. Visualization of reflection of both scattered and collimated light from the different interfaces between air, glass and liquid. 
Since the foam is contained in glass reactors, multiple reflecting surfaces have to be considered when determining the value of the reflection factors for both collimated and diffuse light. The glass walls were considered to be completely wetted by the foam, thus air-glass (a/g), glass-air $(\mathrm{g} / \mathrm{a})$, glass-water $(\mathrm{g} / \mathrm{w})$, and water-glass $(\mathrm{w} / \mathrm{g})$ interfaces were assumed for both collimated $\left(r_{c e}\right)$ and diffuse $\left(r_{21}\right)$ light. The transition between the thin water and the foam was neglected since the water film can be regarded as part of the foam structure. A schematic overview of the light fluxes in the foam-bed photobioreactor are presented in Figure 5.B.1. The $r_{c e}$ was calculated from Snell's law and the Fresnels equations [15], as shown by Eq. 5.B.13. These calculations are based on the refractive indexes of the materials and the incident angles. When the collimated light beam is perpendicular to the surface of the reactor, the collimated light beam hits the airglass $(\mathrm{a} / \mathrm{g})$ interface with an incident angle of $0^{\circ}$. For the calculation of the reflection factor $r_{c e}$, the reflected flux from the a/g interface and the transmitted flux originating from the internal reflection in the glass plate was considered. Further internal reflections in the glass plates were neglected. Similarly, the reflection factor for collimated light at the back of the reactor can be calculated (Eq. 5.B.14).

$r_{c e}=\left(1-r_{a / g}\right) \cdot\left(1-r_{g / a}\right) \cdot r_{g / w}+r_{a / g}$ Equation 5.B.13

$r_{c e}{ }^{\prime}=\left(1-r_{g / w}\right) \cdot\left(1-r_{w / g}\right) \cdot r_{g / a}+r_{w / g}$ Equation 5.B.14

In a similar way $r_{21}$ was calculated for diffuse light (Eq. 5.B.15). Since diffuse light is generated within the foam, inside the reactor, reflection takes place firstly at the water-glass interface and afterwards at the glass-air interface. For the determination of the reflection factor for diffuse light its angular distribution has to be considered. Assuming that the generated diffuse light inside the foam is isotropic, all angles have equal probability. The angular distribution of the transmitted flux and its relative magnitude can be calculated from the incident angles by means of Snell's law and the Fresnel equations. The reflected flux has the same angular-distribution as the incident diffuse light to that interface and thus remains diffuse.

$r_{21}=\left(1-r_{g / w}\right) \cdot\left(1-r_{w / g}\right) \cdot r_{g / a}+r_{w / g}$

Equation 5.B.15

The theory presented by Welch et al. [15] is valid for refractive-index matched boundaries. Since the refractive index of the media (foam) and the environment (glass and air) are different in the case of a foam-bed, refractive index mismatched boundaries have to be considered. Therefore, in this study the refraction of diffuse light from these boundaries was taken into account. In addition, reflection from the boundaries increases the diffuse fluence rate in the foam, therefore the case of index mismatched boundaries is resulting in higher fluence rates 
compared to the matched boundaries. In order to include this extra light flux, we modified the parameters in the theory presented by Welch et al. [15], describing for refractive index matched case. The coefficients $A_{+-}$and $A_{-+}$were altered to fit the boundary conditions of the index mismatched case (Eq. 5.B.21 and 5.B.22). These boundary conditions can be expressed as $F_{+}(0)=r_{21} \cdot F_{-}(0)$ and $F_{-}(d)=r_{21} \cdot F_{+}(d)$, where $\mathrm{r}_{21}$ represents the fraction of light that is reflected back to the scattering media from the boundary. In order to prove the validity of the expressions for $A_{+-}$and $A_{-+}$derived in this study for index mismatch case, they were compared with published data for the index matched boundaries $\left(r_{2 I}=0\right)$. In this case our expression match the expressions presented by Welch et al. [15]. Further variables used in Eq. 5.B.11 and 5.B.12 are presented in Eq. 5.B.16-5.B.23.

$$
\begin{array}{ll}
q(y, \lambda)=\frac{\mu_{e f f}(y, \lambda)-2 \cdot \mu_{a}(y, \lambda)}{\mu_{e f f}(y, \lambda)+2 \cdot \mu_{a}(y, \lambda)} & \text { Equation 5.B.162 } \\
S_{+}(y, \lambda)=\frac{\mu_{s}(y, \lambda)}{4} \cdot\left[\left(5+9 \cdot g_{S}\right) \cdot \mu_{a}(y, \lambda)+5 \cdot \mu_{s}(y, \lambda)\right] & \text { Equation 5.B.17 } \\
S_{-}(y, \lambda)=\frac{\mu_{s}(y, \lambda)}{4} \cdot\left[\left(1-3 \cdot g_{S}\right) \cdot \mu_{a}(y, \lambda)+\mu_{s}(y, \lambda)\right] & \text { Equation 5.B.18 } \\
\left(\mu_{e f f}(y, \lambda)\right)^{2}=3 \cdot \mu_{a}(y, \lambda) \cdot\left[\mu_{a}(y, \lambda)+\left(1-g_{S}\right) \cdot \mu_{s}(y, \lambda)\right] & \text { Equation 5.B.19 } \\
\boldsymbol{\mu}_{\boldsymbol{t}}(\boldsymbol{y}, \lambda)=\boldsymbol{\mu}_{\boldsymbol{a}}(\boldsymbol{y}, \lambda)+\boldsymbol{\mu}_{s}(\boldsymbol{y}, \lambda) & \text { Equation 5.B.20 } \\
A_{ \pm}(y, \lambda)=\frac{-S \pm(y, \lambda)}{\left(\mu_{t}(y, \lambda)\right)^{2}-\left(\mu_{e f f}(y, \lambda)\right)^{2}} & \text { Equation 5.B.21 } \\
A_{+-}(y, \lambda)^{\prime}=\frac{\frac{\left(r_{21} \cdot A_{-}(y, \lambda)-A_{+}(y, \lambda)\right) \cdot\left(1-r_{21} \cdot q(y, \lambda)\right)}{r_{21}-q(y, \lambda)} \cdot \exp \left(\mu_{e f f}(y, \lambda) \cdot d\right)+\left(r_{21} \cdot A_{+}(y, \lambda)-A_{-}(y, \lambda)\right) \cdot \exp \left(-\mu_{t}(y, \lambda) \cdot d\right)}{\frac{\left(1-r_{21} \cdot q(y, \lambda)\right)^{2}}{r_{21}-q(y, \lambda)} \cdot \exp \left(\mu_{e f f}(y, \lambda) \cdot d\right)-\left(r_{21}-q(y, \lambda)\right) \cdot \exp \left(-\mu_{e f f}(y, \lambda) \cdot d\right)}
\end{array}
$$

Equation 5.B.22

$A_{-+}(y, \lambda)^{\prime}=\frac{\left(r_{21} \cdot A_{+}(y, \lambda)-A_{-}(y, \lambda)\right) \cdot \exp \left(-\mu_{t}(y, \lambda) \cdot d\right)-\frac{\left(A_{+}(y, \lambda)-r_{21} \cdot A_{-}(y, \lambda)\right) \cdot\left(r_{21}-q(y, \lambda)\right) \cdot \exp \left(-\mu_{e f f}(y, \lambda) \cdot d\right)}{1-r_{21} \cdot q(y, \lambda)}}{\left(1-r_{21} \cdot q(y, \lambda)\right) \cdot \exp \left(\mu_{e f f}(y, \lambda) \cdot d\right)-\frac{\left(r_{21}-q(y, \lambda)\right)^{2} \cdot \exp \left(-\mu_{e f f}(y, \lambda) \cdot d\right)}{1-r_{21} \cdot q(y, \lambda)}}$

Equation 5.B.23

The optical parameters, such as the scattering and absorption coefficients are both influenced by the liquid fraction, and consequently depend on the vertical location in the reactor. The reduced scattering coefficient of aqueous foams $\left(\mu_{s}{ }^{\prime}\right)$ is the reciprocal of the transport free mean path length $l^{*}(y)$, calculated according to Vera et al. [52], by an empirical formula (Eq. 5.B.24). The light scattering behaviour of microalgae cells was neglected. Similarly, the absorption coefficient $\left(\mu_{a}\right)$ of foam is the reciprocal of the absorption length of the foam, which is related to the absorption length of the liquid phase $\left(l_{a}^{\text {soln }}\right)$, as shown in Eq. 5.B.25. The microalgal cells 
are assumed to be mass culture acclimated when obtaining microalgal absorption cross section, $a_{x}$ values [37]. For the calculations of $\mu_{a}$, the photon distribution between the liquid and gas phase has to be known. According to Gittings et al. [53], at intermediate liquid fractions, in contrast to particularly high or low liquid fractions, a photon does not stay in the gas and liquid phase proportionally to the liquid fraction. This effect is called photon channelling, and described that photons have a preference for the liquid phase in foams. A photon's light path is about $40 \%$ more in the liquid phase compared to a distribution determined by the liquid fraction. Such intermediate liquid fractions are defined between $4 \%$ and $20 \%$, and the corresponding absorption length and absorption coefficient can be expressed by Eq. 5.B.26, while the expressions for particularly low or high liquid fraction values are presented in Eq. 5.B.27.

$\mu_{s}^{\prime}(y)=\mu_{s}(y) \cdot\left(1-g_{s}\right)=\frac{1}{l^{*}(y)}=\frac{1}{2 \cdot r_{b} \cdot\left(1.5+\frac{0.14}{\varepsilon(y)}\right)}$

Equation 5.B.24

$l_{a}^{\text {soln }}(\lambda)=\frac{1}{C_{x} \cdot a_{x}(\lambda)}$

Equation 5.B.25

$\boldsymbol{\mu}_{\boldsymbol{a}}(\boldsymbol{y}, \lambda)=\frac{1}{\boldsymbol{l}_{\boldsymbol{a}}^{\text {soln }}(\lambda) \cdot\left(\frac{0.75}{\varepsilon(y)}+\mathbf{0 . 2 5}\right)}=\frac{\boldsymbol{C}_{x} \cdot \boldsymbol{a}_{x}(\lambda)}{\frac{0.75}{\varepsilon(y)}+0.25} \quad$ for $0.04<\varepsilon<0.2$

Equation 5.B.26

$\mu_{a}(y, \lambda)=\frac{\varepsilon(y)}{l_{a}^{\operatorname{soln}}(\lambda)}=\varepsilon(y) \cdot C_{x} \cdot a_{x}(\lambda) \quad$ for $\varepsilon<0.04$ or $\varepsilon>0.2$

Equation 5.B.27

In order to define the total irradiance, the illuminating light intensities at different wavelengths between 400-700 $\mathrm{nm}$ have to be summed (Eq. 5.B.28), and conversely, the radiation at given wavelength can be calculated based on the normalised spectral distribution of the light source, $E_{n}(\lambda)$ and the total illuminating light intensity $E_{0}$. For the growth simulations, the spectrum of sunlight emission was applied, while for the model validation the spectra of the light source used in our experiments was taken into account.

$E_{0}=\sum_{\lambda=400}^{\lambda=700} E_{0}(\lambda) \cdot \Delta \lambda=\sum_{\lambda=400}^{\lambda=700} E_{0} \cdot E_{n}(\lambda) \cdot \Delta \lambda$

Equation 5.B.28

The light leaving the system via the front wall (where the collimated light beam enters) is the reflected flux, which can be calculated as presented in Eq. 5.B.29. The transmitted flux, which is the light leaving the system on the back of the reactor is the transmitted flux, presented in Eq. 5.B.30.

$$
\begin{array}{ll}
R(y, \lambda)=\left(1-r_{21}\right) \cdot F_{-}(0, y, \lambda)+r_{c e} \cdot E_{0}(\lambda) & \text { Equation 5.B.29 } \\
T(y, \lambda)=\left(1-r_{21}\right) \cdot F_{+}(d, y, \lambda)+\left(1-r_{c e}\right)\left(1-r_{c e}{ }^{\prime}\right) \cdot E_{0}(\lambda) \cdot \exp \left(-\mu_{t}(y, \lambda) \cdot d\right)
\end{array}
$$




\section{B.4 Growth model}

In order to calculate the specific rate of photosynthesis $q_{s}$ (i.e. sugar production in the chloroplast) the model of Jassby \& Platt is applied according to Blanken et al. [10,54]. This model is only applicable if light availability limits the algal growth. In order to predict the local fluence rate, $\phi_{t}(\lambda, y, z)$, the previously described light model was used. Firstly, the biomass specific photon absorption rate $\left(q_{p h}\right)$ is determined by Eq. 5.B.31, which is used to calculate the values of sugar production rate, $q_{s}$ (Eq. 5.B.32) and finally, the local growth rates $(\mu)$ can be obtained (Eq. 5.B.33).

$$
\begin{array}{ll}
q_{p h}(y, z)=\sum_{\lambda=400}^{\lambda=700} a_{x}(\lambda) \cdot \phi_{t}(\lambda, y, z) & \text { Equation 5.B.31 } \\
q_{s}(y, z)=q_{s}{ }^{M A X} \cdot \tanh \left(\frac{Y_{s / p h}{ }^{M A X} \cdot q_{p h}(y, z) \cdot 10^{-6}}{q_{s}{ }^{M A X}}\right) & \text { Equation 5.B.32 } \\
\mu(y, z)=\left(q_{s}(y, z)-m_{s}\right) \cdot Y_{x / s} & \text { Equation 5.B.33 }
\end{array}
$$

In case the biomass concentration is too high, at the darker backside of the reactor negative production rates will develop because of biomass loss due to maintenance (endogenous respiration). However, due to the assumption of an ideally mixed liquid phase, these local rates can be averaged for the whole liquid phase volume (Eq. 5.B.34).

$\mu^{a v}=\frac{\iint_{z=o, y=0}^{z=d, y=h} \mu(y, z) \cdot \varepsilon(y) \cdot d y \cdot d z \cdot w}{V^{L}}$

Equation 5.B.34

Where the total amount of liquid in the reactor is expressed by Eq. 5.B.35.

$V^{L}=d \cdot w \cdot \int_{y=0}^{y=h} \varepsilon(y) \cdot d y$

Equation 5.B.35

The liquid flow rate for the continuous operation is expressed based on the fact that in the dilution rate equals the growth rate (Eq. 5.B.36).

$F^{L}=\mu^{a v} \cdot V^{L}$

Equation 5.B.36

The reactor productivity $\left(\mathrm{mol}_{\mathrm{x}} \mathrm{m}^{-3} \mathrm{~s}^{-1}\right)$ can be obtained by Eq. 5.B.37, while the reactor areal productivity by Eq. 5.B.38, expressed as $\mathrm{g} \mathrm{m}^{-2} \mathrm{~d}^{-1}$.

$r_{x}^{L, a v}=\mu^{a v} \cdot C_{x}$

Equation 5.B.37

$r_{x}^{A r e a l}=\frac{\mu^{a v} \cdot C_{x} \cdot M W_{x} \cdot V^{L} \cdot 3600 \cdot 24}{A^{l i g h t}}$

Equation 5.B.38

The biomass yield on light energy in the units of $\mathrm{g} \mathrm{mol}_{\mathrm{ph}}{ }^{-1}$ can be calculated by Eq. 5.B.39. 
$Y_{x / p h}=\frac{r_{x}^{L, a v} \cdot C_{x} \cdot M W_{x} \cdot V^{L}}{10^{-6} \cdot E_{0} \cdot A^{l i g h t}}$

Equation 5.B.39

The mass transfer model is linked to the growth model via a linear correlation between the light dependent biomass production rate, $r_{x}$, and the $\mathrm{CO}_{2}$ and $\mathrm{O}_{2}$ production rates, $r_{\mathrm{CO}_{2}}$ and $r_{\mathrm{O} 2}$. According to the stoichiometry of the growth of Chlorella (Eq. 5.B.40), with the given cell composition $\left(\mathrm{CH}_{1.78} \mathrm{O}_{0.36} \mathrm{~N}_{0.12} \mathrm{P}_{0.01}\right)$ and nutrients in the media (urea as nitrogen source, and phosphate as phosphorus source), the yield of $\mathrm{CO}_{2}$ on biomass $\left(\mathrm{Y}_{\mathrm{CO} 2 / x}\right)$ and the yield of $\mathrm{O}_{2}$ on biomass $\left(Y_{O 2 / x}\right)$ can be calculated.

$0.94 \mathrm{CO}_{2}+0.06 \mathrm{CH}_{4} \mathrm{~N}_{2} \mathrm{O}+0.01 \mathrm{H}_{2} \mathrm{PO}_{4}^{-}+0.755 \mathrm{H}_{2} \mathrm{O}+0.01 \mathrm{H}^{+} \rightarrow$ $\mathrm{CH}_{1.78} \mathrm{O}_{0.36} \mathrm{~N}_{0.12} \mathrm{P}_{0.01}+1.1875 \mathrm{O}_{2}$

Equation 5.B.40

These yields can be used to calculate the average liquid phase volumetric production rates $\left(r_{i}^{L, a v}\right.$, where $i$ stands for either $\mathrm{O}_{2}$ or $\mathrm{CO}_{2}$ ) from the average biomass productivity, $r_{x}^{L, a v}$, according to Eq. 5.B.41.

$r_{i}^{L, a v}=r_{x}^{L, a v} \cdot Y_{\frac{i}{x}}$

Equation 5.B.41

\section{B.5 Mass transfer model}

In order to describe the mass transfer processes taking place in the foam, the mass transfer coefficient is essential. Since the gas phase resistance is negligible compared to the liquid phase resistance [16], the overall mass transfer coefficient equals to the liquid side mass transfer coefficient. The liquid side mass transfer coefficient can be evaluated from the formula established for packed bed reactors containing closely-packed solid spheres [55], as shown in Eq. 5.B.42. This formula describes well the mass transfer in the foam, as proven by Perry et al. [16].

$k_{L}(y)=\left[2+1.1 \cdot\left(\frac{\rho \cdot V_{s l i p}(y) \cdot 2 \cdot r_{b}}{\mu_{L}}\right)^{0.6} \cdot\left(\frac{\mu_{L}}{\rho \cdot Ð}\right)^{1 / 3}\right] \frac{Ð}{2 \cdot r_{b}}$

Equation 5.B.42

In Eq. 5.B.42 the slip velocity $V_{\text {slip }}$ represents the difference between the gas and liquid velocities in the rising foam [14,16], as shown in Eq. 5.B.43. The liquid velocity can be obtained by applying the steady state liquid flux presented in the liquid fraction model.

$V_{\text {slip }}(y)=\frac{J_{G}}{1-\varepsilon}-\frac{J_{f}}{\varepsilon}$

Equation 5.B.43

The expression for the specific surface area, $a$ is based on expressing the amount of bubbles with a radius of $r_{b}$ in a volume element $d V^{R}$, and calculating the total surface present in a volume $d V^{R}$ (Eq. 5.B.44). 
$a(y)=\frac{4 \cdot \pi \cdot r_{b}^{2}}{d V^{R}} \cdot \frac{d V^{R} \cdot(1-\varepsilon(y))}{0.75 \cdot \pi \cdot r_{b}{ }^{3}}=\frac{3 \cdot(1-\varepsilon(y))}{r_{b}}$

Equation 5.B.44

The specific surface area only changes linearly with the liquid fraction when spherical bubbles are assumed. In case of dry foams bubble deformation becomes more dominant, thus the specific surface area increases rapidly with decreasing liquid fraction. A more precise estimate of $a$ could be achieved by taking into account the osmotic pressure of the foam [14,23]. In case of low liquid fractions, further deviations from Eq. 5.B.44 might occur since in dry foams mainly Plateau borders and nodes are involved in the mass transfer, thus only a fraction of the bubble surface area might participate in the mass transfer [16, 24].

In order to describe the $\mathrm{O}_{2}$ and $\mathrm{CO}_{2}$ mass transfer in the foam, a mass balance for those components over both the liquid and gas phases (combined balance) is set up (Eq. 5.B.45). These mass balances are described over horizontal infinitesimally small slices of the reactor, expressing the flow of component $i$ to and from the slice via the gas phase and the transfer rate of the component between the gas and liquid phase within the slice. Due to the assumption of ideally mixed liquid phase, liquid concentrations are the same in all slices. Additionally, the dilution rate for the continuous operation was taken into account for the liquid phase of each individual slice. In case the $\mathrm{CO}_{2}$ concentration in the inlet liquid flow rate, $c_{C O 2}{ }^{L, I N}$ is the same as the outflow $\mathrm{CO}_{2}$ concentration $c_{\mathrm{CO}^{2}}{ }^{L}$, the last term in Eq. 5.B.45 can be neglected. The effect of the mass transfer on the total volumetric gas flow is neglected, since the $\mathrm{CO}_{2}$ uptake and $\mathrm{O}_{2}$ production rate are similar. The gas phase gradient in height can be expressed by Eq. 5.B.46.

$0=-F^{G} \cdot d c_{i}^{G}(y)+k_{L} a(y) \cdot\left(c_{i}^{*}(y)-c_{i}^{L}\right) \cdot A \cdot d y+\frac{F^{L} \cdot A \cdot \varepsilon(y) \cdot d y}{V^{L}} \cdot\left(c_{i}{ }^{L, i n}-c_{i}{ }^{L}\right)$

Equation 5.B.45

$\frac{d c_{i}^{G}(y)}{d y}=\frac{k_{L} a(y) \cdot\left(c_{i}^{*}(y)-c_{i}^{L}\right) \cdot A+\frac{F^{L} \cdot A}{V^{L}} \cdot \varepsilon(y) \cdot\left(c_{i}^{L, i n}-c_{i}^{L}\right)}{F^{G}}$

Equation 5.B.46

For the liquid phase an overall reactor (macro)balance can be used as the liquid phase is ideally mixed, therefore liquid concentrations are constant at every location. Thus, the total amount of component $i$ transferred between the gas and liquid phase can be described as the sum of the transferred amounts at each height. The total gas transferred equals to the production/uptake rate of that compound in the liquid phase of the reactor plus the additional flow of the compound due to continuous reactor operation and the resulting liquid flow rate $\left(F_{L}\right)$, as described in Eq. 5.B.47. 
$0=\int_{0}^{h} k_{L} a(y) \cdot\left(c_{i}^{*}(y)-c_{i}^{L}\right) \cdot A \cdot d y+r_{i}^{L, A V G} \cdot V^{L}+F^{L} \cdot\left(C_{i}^{L, i n}-C_{i}^{L}\right) \quad$ Equation 5.B.47

To express the concentration difference determining the driving force for the gas-liquid mass transfer, instead of the gas phase concentration of a certain compound, the interfacial concentration $\left(c_{i}{ }^{*}\right)$ is applied since the liquid at the gas-liquid interface is in equilibrium with the gas phase (Eq. 5.B.45-5.B.47). Thus, for calculating the concertation in liquid phase near the gas-liquid interface Henry's law is used, which relates the partial pressure of the compound in the gas phase to its concentration in the liquid phase via Henry's coefficient (Eq. 5.B.48). The partial pressure is expressed as the total pressure multiplied with the gas molar fraction, which is the concentration of compound $i$ in the gas phase divided by the molar density $(M D)$ of the gas at $37^{\circ} \mathrm{C}$.

$c_{i}^{*}=\frac{P i}{H_{i}}=\frac{P_{T} \cdot y_{i}}{H_{i}}=\frac{P_{T}}{H_{i}} \cdot \frac{C_{i}^{G}}{M D}$

Equation 5.B.48

The liquid phase concentration of $\mathrm{CO}_{2}$ required is determined by the $K_{s}$ value. We selected a minimum $\mathrm{CO}_{2}$ concentration at five times the $K_{s}$ value. This $K_{s}$ value was determined by Shelp and Canvin at neutral $\mathrm{pH}$ and is based on $\mathrm{CO}_{2}$ only [49]. The liquid flow rate for the continuous operation was then assumed to contain $\mathrm{HCO}_{3}{ }^{-}$which is in equilibrium with the $5 \cdot K_{s} \mathrm{CO}_{2}$ concentration at the operational $\mathrm{pH}$ (6.7 for Chlorella sorokiniana), and therefore the inlet liquid also contains $5 \cdot K s \mathrm{CO}_{2}$. To achieve this desired liquid $\mathrm{CO}_{2}$ concentration, the inlet gas $\mathrm{CO}_{2}$ concentration requirements, $\mathrm{CCO}_{2}{ }^{\mathrm{Gin}}$ can be predicted by Eq. 5.B.46 and 5.B.47.

The efficiency of $\mathrm{CO}_{2}$ uptake, representing the fraction of supplied $\mathrm{CO}_{2}$ taken up by microalgae, was calculated by Eq. 5.B.49.

$\eta_{\mathrm{CO2} 2}=\frac{\left(C_{\mathrm{CO2}}^{G, i n}-C_{\mathrm{CO2}}^{G, \text { out }}\right)}{C_{\mathrm{CO} 2}^{G, \text { in }}}$

Equation 5.B.49

For $\mathrm{O}_{2}$, the inlet gas concentration $\mathrm{C}_{\mathrm{O}_{2}}^{G, i n}$ can be calculated assuming air enriched with the above calculated concentration of $\mathrm{CO}_{2}$. The inlet liquid medium during the continuous operation is assumed to be saturated with air. Therefore the eventual liquid $\mathrm{O}_{2}$ concentration can be determined, by several iterative cycles. Initially the assumption was made that in Eq. 5.B.46, $\mathrm{CO}_{2}{ }^{L}=\mathrm{CO}_{2}{ }^{L, \text { in }}$ since in the flow for continuous operation the $\mathrm{O}_{2}$ concentrations are really low compared to the gas phase concentrations. By this assumption, the gas phase $\mathrm{O}_{2}$ concentration gradient could be calculated, which is used to determine the liquid phase concentration by Eq. 5.B.50, and the resulting liquid phase concentration was used in the next cycle. 
$C_{i}^{L}=\frac{r_{i}^{L, A V G} \cdot V^{L}+F^{L} \cdot C_{i}^{L, I N}+\int_{0}^{h} k_{L} a(y) \cdot C_{i}^{*}(y) \cdot A \cdot d y}{F^{L}+\int_{0}^{h} k_{L} a(y) \cdot A \cdot d y}$

Equation 5.B.50

\section{B.6 Energy Calculations}

The energy requirement of the simulated photobioreactor designs and operational conditions are determined by the gas blowers, liquid pumps to recirculate the liquid from the bottom to the top of the reactor, and the operation of a centrifuge for algae separation.

For the power requirement for pumping liquid to a higher level the hydrostatic pressure, the liquid recirculation rate, and the pump efficiency was considered, as shown in Eq. 5.B.51.

$P^{L}=\frac{F^{R \cdot h \cdot \rho \cdot g}}{\eta_{L p u m p}}$

Equation 5.B.51

In order to aerate the photobioreactor, an air compressor or blower can be used. When adiabatic compression is applied on a di-atiomic gas, the power requirements expressed in watts can be calculated according to Eq. 5.B.52, in which the pump efficiency is incorporated. The pressure of the compressed gas has to equal to the hydrostatic pressure of the water column, therefore $p_{F}=\rho \cdot h \cdot g+P^{T}$, with all pressure units expressed in $\mathrm{Pa}$.

$P^{G}=\frac{-2.5 \cdot p_{T^{\prime}} \cdot F^{G}\left(1-\left(\frac{p_{F}}{p_{T}}\right)^{\frac{2}{7}}\right)}{\eta_{\text {Gpump }}}$

Equation 5.B.52

The energy requirement for the algal separation per gram of dry algae produced $\left(E^{S e p,} \mathrm{~kJ} \mathrm{~g}^{-1}\right)$ was calculated assuming $1.5 \mathrm{kWh}\left(5.4 \cdot 10^{6}\right.$ joules $)$ required to process $1 \mathrm{~m}^{3}$ of algal suspension with plate separators [19], as shown in Eq. 5.B.53 as $E^{C x S e p}$, and $C_{x}$ is expressed as $\mathrm{g} \mathrm{L}^{-1}$.

$E^{S e p}=\frac{E^{C x S e p} \cdot 10^{-6}}{C_{x}}=\frac{5.4}{C_{x}}$

Equation 5.B.53

The total energy requirements per gram of algae produced $\left(E, \mathrm{~kJ} \mathrm{~g}^{-1}\right)$ are therefore the sum of the above mentioned energy requirements for the liquid pump, gas blower, and algae separator, as shown in Eq. 5.B.54 $\left(r_{x}^{L, a v}\right.$ in $\mathrm{mol}_{\mathrm{x}} \mathrm{m}^{-3} \mathrm{~s}^{-1}, V^{L}$ in $\mathrm{m}^{3}, P^{L}$ and $P^{G}$ in W).

$E=\frac{P^{L}+P^{G}}{r_{x}^{L, a v} \cdot M W_{x} \cdot V^{L}}+E^{S e p}$

Equation 5.B.54 


\section{References}

[1] R.H. Wijffels, M.J. Barbosa, M.H. Eppink, Microalgae for the production of bulk chemicals and biofuels, Biofuels, Bioprod. Biorefin. 4 (2010) 287-295.

[2] J. Ruiz, G. Olivieri, J. de Vree, R. Bosma, P. Willems, J.H. Reith, M.H. Eppink, D.M. Kleinegris, R.H. Wijffels, M.J. Barbosa, Towards industrial products from microalgae, Energy Environ. Sci. 9 (2016) 3036-3043.

[3] R.N. Singh, S. Sharma, Development of suitable photobioreactor for algae production - A review, Renew. Sust. Energ. Rev. 16 (2012) 2347-2353.

[4] A. Janoska, P.P. Lamers, A. Hamhuis, Y. van Eimeren, R.H. Wijfels, M. Janssen, A liquid foam-bed photobioreactor for microalgae production, Chem. Eng. J. 313 (2017) 1206-1214.

[5] P. Stevenson, Hydrodynamic theory of rising foam, Miner. Eng. 20 (2007) 282-289.

[6] N. Isert, G. Maret, C.M. Aegerter, Coarsening dynamics of three-dimensional levitated foams: From wet to dry, Eur. Phys. J. E 36 (2013) 116-121.

[7] A.-L. Biance, A. Delbos, O. Pitois, How topological rearrangements and liquid fraction control liquid foam stability, Phys. Rev. Lett. 106 (2011) 068301.

[8] A. Janoska, R. Barten, S. de Nooy, P. van Rijssel, René H. Wijfels, M. Janssen, Improved liquid foam-bed photobioreactor design for microalgae cultivation, Algal Res. submitted.

[9] A. Janoska and M. Vázquez, M.Janssen, R. H. Wijffels, M. Cuaresma, C. Vílchez, Surfactant selection for a liquid foam-bed photobioreactor, Biotechnol. Prog. in press.

[10] W. Blanken, P.R. Postma, L. de Winter, R.H. Wijffels, M. Janssen, Predicting microalgae growth, Algal Res. 14 (2016) 28-38.

[11] R. Kant Sharma, A. Gaikwad, A.N. Bhaskarwar, Gas Absorption with Chemical Reaction and Desorption in a Foam-bed Reactor, Chem. Eng. Commun. 192 (2005) 597-619.

[12] E. Kan, M.A. Deshusses, Modeling of a foamed emulsion bioreactor: I. Model development and experimental validation, Biotechnol. Bioeng. 99 (2008) 1096-1106.

[13] P. Ireland, R. Cunningham, G.J. Jameson, The behaviour of wash water injected into a froth, Int. J. Miner. Process. 84 (2007) 99-107.

[14] P. Yazhgur, E. Rio, F. Rouyer, F. Pigeonneau, A. Salonen, Drainage in a rising foam, Soft matter 12 (2016) 905-913.

[15] A.J. Welch, M.J. Van Gemert, Optical-thermal response of laser-irradiated tissue, second ed., Springer, Dordrecht, 2011.

[16] D.C. Perry, P. Stevenson, Gas absorption and reaction in a wet pneumatic foam, Chem. Eng. Sci. 126 (2015) 177-185.

[17] A.M. Kliphuis, D.E. Martens, M. Janssen, R.H. Wijffels, Effect of O2: $\mathrm{CO} 2$ ratio on the primary metabolism of Chlamydomonas reinhardtii, Biotechnol. Bioeng. 108 (2011) 2390-2402.

[18] S.M. Walas, Chemical Process Equipment-Selection and Design, Butterworth-Heinemann, USA, 1990.

[19] J. Doucha, K. Lívanský, Novel outdoor thin-layer high density microalgal culture system: Productivity and operational parameters, Algol. Stud. Supplement 76 (1995) 129-147.

[20] J. Doucha, F. Straka, K. Livanský, Utilization of flue gas for cultivation of microalgae (Chlorella sp.) in an outdoor open thin-layer photobioreactor, J. Appl. Phycol. 17 (2005) 403-412.

[21] R. Reyna-Velarde, E. Cristiani-Urbina, D.J. Hernández-Melchor, F. Thalasso, R.O. Cañizares-Villanueva, Hydrodynamic and mass transfer characterization of a flat-panel airlift photobioreactor with high light path, Chem. Eng. Process.: Process Intensification 49 (2010) 97-103.

[22] T.M. Sobczuk, F.G. Camacho, F.C. Rubio, F. Fernandez, E.M. Grima, Carbon dioxide uptake efficiency by outdoor microalgal cultures in tubular airlift photobioreactors, Biotechnol. Bioeng. 67 (2000) 465-475.

[23] R. Höhler, Y. Yip Cheung Sang, E. Lorenceau, S. Cohen-Addad, Osmotic pressure and structures of monodisperse ordered foam, Langmuir 24 (2008) 418-425.

[24] O. Pitois, E. Lorenceau, N. Louvet, F. Rouyer, Specific surface area model for foam permeability, Langmuir 25 (2008) 97-100.

[25] M. Bouaifi, G. Hebrard, D. Bastoul, M. Roustan, A comparative study of gas hold-up, bubble size, interfacial area and mass transfer coefficients in stirred gas-liquid reactors and bubble columns, Chem. Eng. Process.: Process Intensification 40 (2001) 97-111.

[26] E. Sierra, F.G. Acién, J.M. Fernández, J.L. García, C. González, E. Molina, Characterization of a flat plate photobioreactor for the production of microalgae, Chem. Eng. J. 138 (2008) 136-147. 
[27] C.J. Hulatt, D.N. Thomas, Productivity, carbon dioxide uptake and net energy return of microalgal bubble column photobioreactors, Bioresour. Technol. 102 (2011) 5775-5787.

[28] C. Posten, Design principles of photo-bioreactors for cultivation of microalgae, Eng. Life Sci. 9 (2009) 165177.

[29] E. Molina, J. Fernández, F. Acién, Y. Chisti, Tubular photobioreactor design for algal cultures, J. Biotechnol. 92 (2001) 113-131.

[30] J.C. Weissman, R.P. Goebel, J.R. Benemann, Photobioreactor design: mixing, carbon utilization, and oxygen accumulation, Biotechnol. Bioeng. 31 (1988) 336-344.

[31] H. Takache, G. Christophe, J.F. Cornet, J. Pruvost, Experimental and theoretical assessment of maximum productivities for the microalgae Chlamydomonas reinhardtii in two different geometries of photobioreactors, Biotechnol. Progr. 26 (2010) 431-440.

[32] H. Qiang, A. Richmond, Productivity and photosynthetic efficiency of Spirulina platensis as affected by light intensity, algal density and rate of mixing in a flat plate photobioreactor, J. Appl. Phycol. 8 (1996) 139-145.

[33] S.A. Koehler, S. Hilgenfeldt, H. Stone, Foam drainage on the microscale: I. Modeling flow through single plateau borders, J. Colloid Interface Sci. 276 (2004) 420-438.

[34] A. Wileman, A. Ozkan, H. Berberoglu, Rheological properties of algae slurries for minimizing harvesting energy requirements in biofuel production, Bioresour. Technol. 104 (2012) 432-439.

[35] B. Haffner, Y. Khidas, O. Pitois, The drainage of foamy granular suspensions, J. Colloid Interface Sci. 458

(2015) 200-208.

[36] M. Cuaresma, M. Janssen, C. Vilchez, R.H. Wijfels, Productivity of Chlorella sorokiniana in a short light-path

(SLP) panel photobioreactor under high irradiance, Biotechnol. Bioeng. 104 (2009) 352-359.

[37] T. de Mooij, Z.R. Nejad, L. van Buren, R.H. Wijffels, M. Janssen, Effect of photoacclimation on microalgae mass culture productivity, Algal Res. 22 (2017) 56-67.

[38] K. Tuantet, H. Temmink, G. Zeeman, M. Janssen, R.H. Wijffels, C.J. Buisman, Nutrient removal and microalgal biomass production on urine in a short light-path photobioreactor, Water Res. 55 (2014) 162-174.

[39] A.M. Kliphuis, L. de Winter, C. Vejrazka, D.E. Martens, M. Janssen, R.H. Wijffels, Photosynthetic efficiency of Chlorella sorokiniana in a turbulently mixed short light-path photobioreactor, Biotechnol. Progr. 26 (2010) 687696.

[40] University of Reading, Calculator to estimate the density and viscosity of glycerine / water mixtures http://www.met.reading.ac.uk/ sws04cdw/viscosity_calc.html, 2017 (accessed 30 January 2018)

[41] H.C. Cheng, R. Lemlich, Errors in the measurement of bubble size distribution in foam, Ind. Eng. Chem. Fundam. 22 (1983) 105-109.

[42] R. Shaw, G.M. Evans, P. Stevenson, Start-up transients in a pneumatic foam, Asia-Pac. J. Chem. Eng. 6 (2011) 613-623.

[43] N. Vargaftik, B. Volkov, L. Voljak, International tables of the surface tension of water, J. Phys. Chem. Ref. Data 12 (1983) 817-820.

[44] R. Sander, Compilation of Henry's law constants (version 4.0) for water as solvent, Atmos. Chem. Phys. 15 (2015) 4399-4981.

[45] W. Lu, H. Guo, I.-M. Chou, R. Burruss, L. Li, Determination of diffusion coefficients of carbon dioxide in water between 268 and $473 \mathrm{~K}$ in a high-pressure capillary optical cell with in situ Raman spectroscopic measurements, Geochim. Cosmochim. Acta 115 (2013) 183-204.

[46] P. Han, D.M. Bartels, Temperature dependence of oxygen diffusion in $\mathrm{H}_{2} \mathrm{O}$ and $\mathrm{D}_{2} \mathrm{O}$, J. Phys. Chem. 100 (1996) 5597-5602.

[47] P. Duboc, I. Marison, U. Von Stockar, Quantitative calorimetry and biochemical engineering, in: R.B. Kemp (Ed.), Handbook of thermal analysis and calorimetry Vol. 4: From Macromolecules to Man, First edition, Elsevier, Amsterdam, 1999, pp. 267-365

[48] The Engineering ToolBox, Refractive Index for some common Liquids, Solids and Gases.

http://www.engineeringtoolbox.com/refractive-index-d_1264.html (accessed 30 January 2018)

[49] B.J. Shelp, D.T. Canvin, Utilization of exogenous inorganic carbon species in photosynthesis by Chlorella pyrenoidosa, Plant Physiol. 65 (1980) 774-779.

[50] P. Brimblecombe, Air composition and chemistry, Cambridge University Press, Cambridge, 1996.

[51] F. Rouyer, O. Pitois, E. Lorenceau, N. Louvet, Permeability of a bubble assembly: From the very dry to the wet limit, Phys. Fluids 22 (2010) 043302.

[52] M.U. Vera, A. Saint-Jalmes, D.J. Durian, Scattering optics of foam, Appl. Opt. 40 (2001) 4210-4214.

[53] A. Gittings, R. Bandyopadhyay, D. Durian, Photon channelling in foams, EPL 65 (2004) 414. 
[54] A.D. Jassby, T. Platt, Mathematical formulation of the relationship between photosynthesis and light for phytoplankton, Limnol. Oceanogr. 21 (1976) 540-547.

[55] N. Wakao, S. Kaguei, T. Funazkri, Effect of fluid dispersion coefficients on particle-to-fluid heat transfer coefficients in packed beds: correlation of Nusselt numbers, Chem. Eng. Sci. 34 (1979) 325-336. 

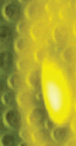

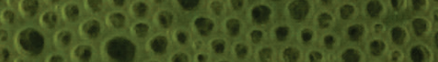

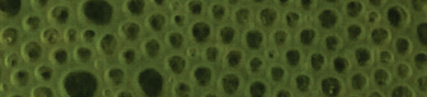

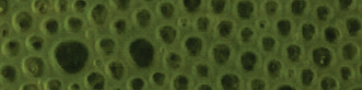

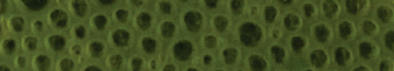
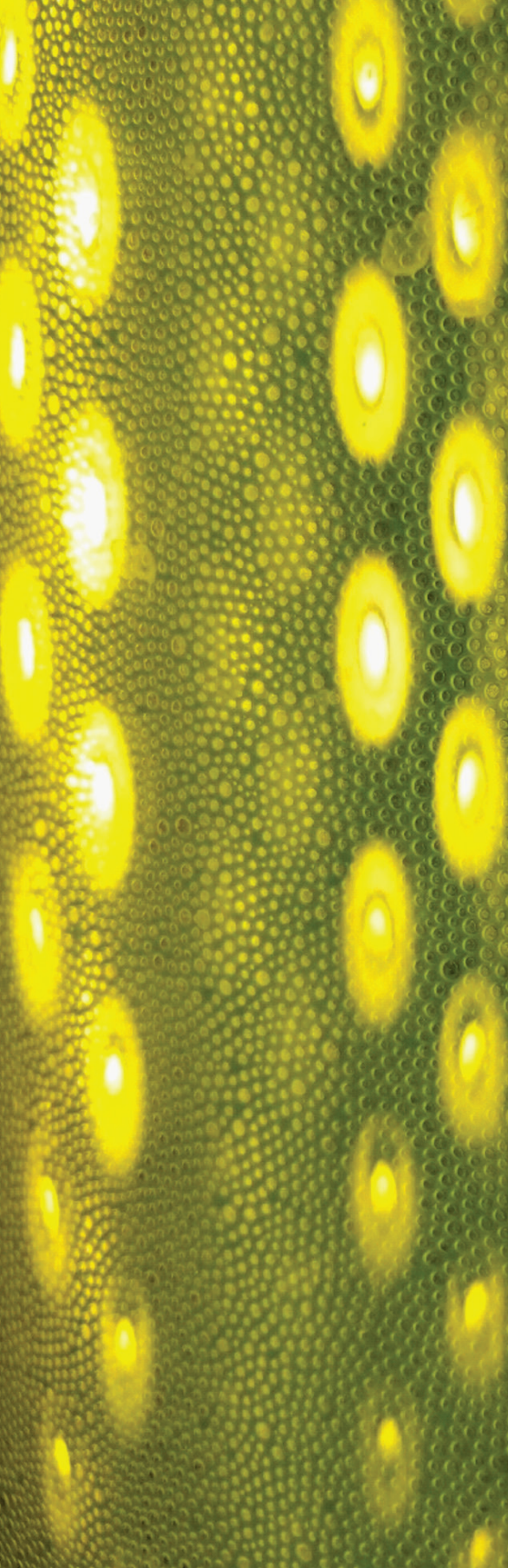

og. है?

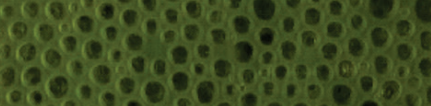

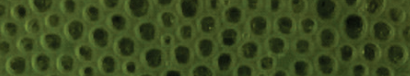

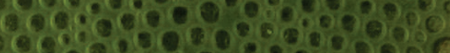

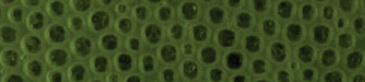
$109898: 800$
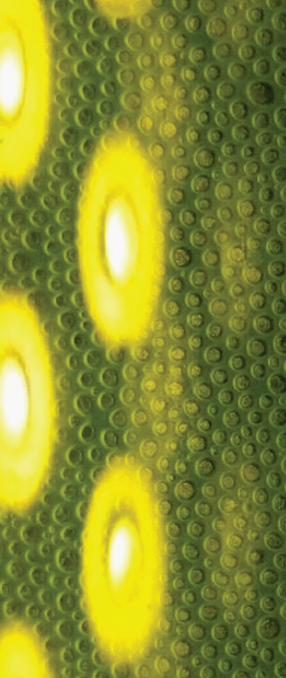

$989: 8: 90: 8$

$68: 0398000$
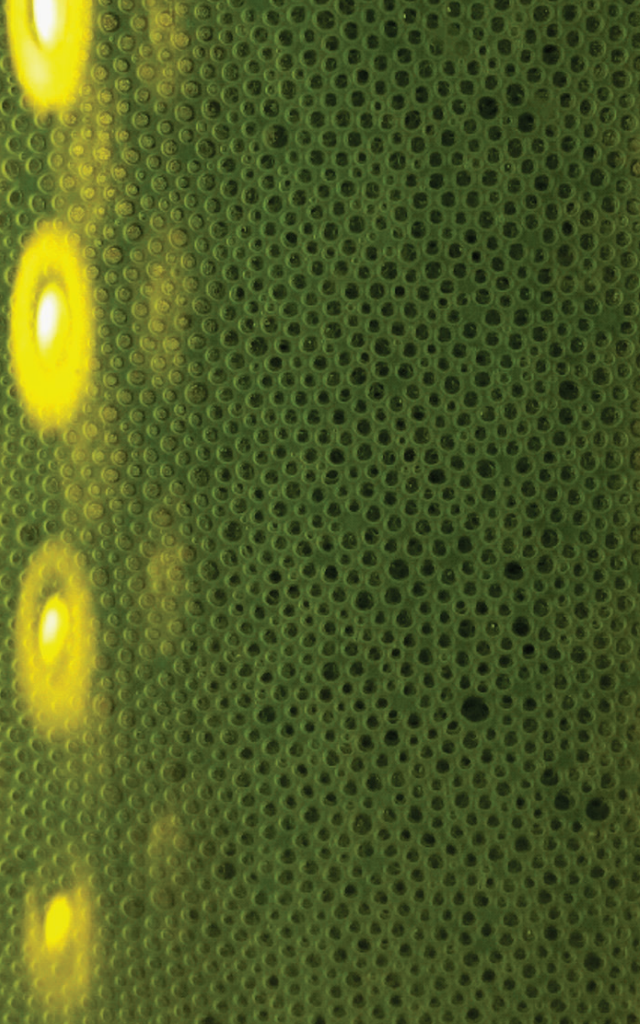

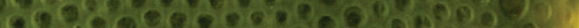

0

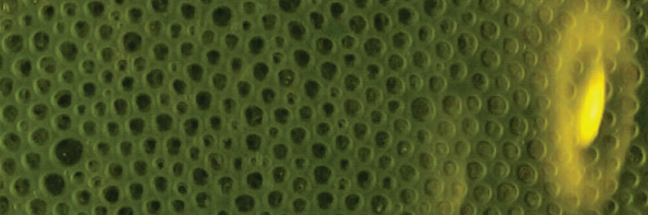


Chapter 6

General discussion 


\subsection{Advantages of a liquid foam-bed photobioreactor}

A liquid foam-bed photobioreactor holds several advantages compared to conventional, liquid phase photobioreactors for microalgae production. These advantages include the following:

A) Increased gas transfer rate resulting in reduced gas supply requirements

B) Low pressure drop leading to reduced gassing energy requirement and reduced material requirements due to the low hydrostatic pressure of the foam column

C) Short light absorption path allowing for cultivation at high biomass densities

\section{A) Gas transfer rate}

The gas transfer rate between the gas and liquid phases in the foam-bed photobioreactor is described by the volumetric mass transfer coefficient $\left(k_{L} a\right)$. The measured $k_{L} a$ value in our labscale foam-bed photobioreactor was $0.108-0.135 \mathrm{~s}^{-1}$, depending on the operational conditions. This $k_{L} a$ value is 6 fold higher compared to the $k_{L} a$ obtained in case the foam-bed photobioreactor was operated as a bubble column. The improvement of the mass transfer coefficient is even higher when comparing to $k_{L} a$ values determined in other studies [1]. The $k_{L} a$ value is the product of the liquid side mass transfer coefficient and the specific surface area. Since the foam offers a large interfacial area between the gas and liquid phases, the $k_{L} a$ values will be higher compared to conventional, liquid phase photobioreactors. The specific surface area in our lab-scale liquid foam-bed photobioreactor was $7142 \mathrm{~m}^{-1}$, which is 10 to 140 fold higher than in a bubble column reported by Perry et al. [2]. Although the specific surface area in foam-bed reactors is 10-140 times higher than in bubble columns the overall mass transfer coefficient is only 6 times higher. Apparently the mass transfer coefficient, $k_{L}$, in foam-beds is lower than in bubble column reactors. This reduction of the $k_{L}$ is related to the low superficial velocities [3], small bubble sizes and the additional resistance created by the surfactants leading to less a mobile interface and to a reduced diffusion coefficient in foam-beds $[4,5]$.

This increased mass transfer rate allows for the application of reduced gassing rates in the foambed photobioreactor compared to conventional, flat panel photobioreactors. The superficial gas flow rate during our lab-scale experiments was $0.16-0.62 \mathrm{~mm} \mathrm{~s}^{-1}$. This superficial velocity is substantially lower compared to the ones applied in flat panel photobioreactors. For example, in a flat panel described by Ruiz et al. [6] a gassing rate of $0.32 \mathrm{vvm}$ is applied, translating to $2.7 \mathrm{~mm} \mathrm{~s}^{-1}$ superficial velocity. 


\section{B) Low pressure drop}

The low liquid fraction of the foam, which ranges from 1 to $15 \%$, ensures that the hydrostatic pressure of the foam column is much lower compared to water-filled flat panel photobioreactors. The measured liquid fraction in the foam-bed under the experimental conditions described in Chapter 4, applying $0.59 \mathrm{~mm} \mathrm{~s}^{-1}$ liquid recirculation superficial velocity, was approximately $12 \%$. The resulting hydrostatic pressure of a foam-bed is more than 8 times lower compared to a flat-panel photobioreactor of the same height. Therefore, the carrier capacity of the structure supporting the photobioreactors can be considerably lower saving on construction costs of large-scale systems. Moreover, this low pressure drop in the reactor will also lead to a large reduction in energy costs for aeration.

\section{C) High biomass densities}

One of the main advantages of the foam-bed photobioreactor is that increased biomass densities can be reached compared to traditional photobioreactors. When adopting flat-panel photobioreactors in combination with a liquid foam-bed the light path is in the order of a few millimetres only. Consequently, the biomass concentration can be increased with one order of magnitude, reducing downstream processing costs. During our experimental studies, biomass densities between 20 and $25 \mathrm{~g} \mathrm{~L}^{-1}$ were obtained during chemostat cultivation experiments. This biomass concentration is substantially higher than the biomass concentration in conventional systems. Generally, in flat panel systems biomass concentrations between 2 and $3 \mathrm{~g} \mathrm{~L}^{-1}$ can be obtained while tubular photobioreactors are operated between 1 and $2 \mathrm{~g} \mathrm{~L}^{-1}$ biomass [6, 7]. In order to approach the biomass densities achieved in the foam-bed photobioreactor with traditional flat panel photobioreactors, a panel of only $2 \mathrm{~mm}$ depth should be built (Figure 6.1). However, practical limitations of gas transfer restricts the application of such thin reactors. Flat panels are usually 2-7 $\mathrm{cm}$ deep $[6,8]$, and consequently the maximum achieved biomass concentration in those reactors are lower. 


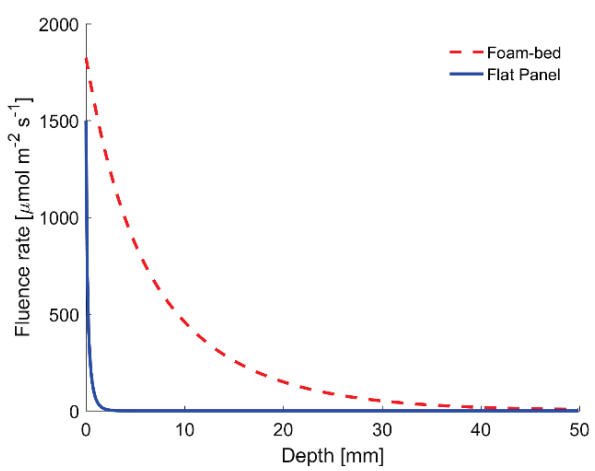

Figure 6.1. Model predictions of the fluence rate profile in a foam-bed and a flat panel under the same conditions: biomass density $30 \mathrm{~g} \mathrm{~L}^{-1}$ and light intensity $1500 \mu \mathrm{mol} \mathrm{m} \mathrm{m}^{-2} \mathrm{~s}^{-1}$. The light attenuation in a flat panel was modelled according to Lambert-Beers law.

\subsection{Foam-bed photobioreactor design}

The design of a liquid foam-bed photobioreactor is of key importance as it determines its productivity and energy requirements. In this thesis, two different foam-bed designs were tested. In the first design, the foam was lead from the top of the reactor to an external foam breaker, where the liquid phase of the foam was separated from the gas entrapped in the bubbles. The microalgal suspension was pumped back to the bottom of the reactor, where it was incorporated again in freshly formed foam. This design ensured continuous foaming for 8 hours and it was successful for microalgae cultivation when BSA was applied as a foam stabilising agent [9].

The second reactor design was developed in order to i) compensate for the low microalgae partitioning to the foam phase when Pluronic F68 is applied as a surfactant, ii) enhance foam stability, iii) enable homogeneous surfactant distribution, and iv) eliminate the need of an external foam breaker. In this second design, the microalgae suspension was continuously pumped from the bottom of the reactor to the top of the foam column, where it was allowed to drain down again. This liquid recirculation allowed for homogeneous algae distribution in the foam column, thereby enhancing the algae concentration in foams stabilized by Pluronic F68. This liquid recirculation also gave the opportunity to break the foam on the top of the foam column by the liquid jets entering into the reactor. The additional liquid drainage also enhances the mixing in the foam, which is beneficial in order to improve mass transfer and avoid $\mathrm{pH}$ or dissolved $\mathrm{O}_{2} / \mathrm{CO}_{2}$ concentration gradients along the column. 
Liquid recirculation is also required to increase foam stability. In preliminary experiments applying Pluronic F68 without liquid recirculation in the foam-bed photobioreactor, the foam stability decreased rapidly in time, and continuous foam formation could not be maintained. Due to the liquid recirculation, also surfactant accumulation in the top foam layers can be prevented. Surfactant molecules accumulate on the top of the foam column in case the foam is broken at the top [10]. This is because the surfactants adsorbed on the gas-liquid interfaces are released from the bursting bubbles, and their drainage back to the bulk liquid layer underneath the foam column is not efficient. When liquid recirculation is applied, these free surfactant molecules return rapidly to the bulk liquid layer with the solution draining down through the column. The resulting homogenous surfactant distribution is a prerequisite for continuous foam formation. Additionally, when liquid recirculation is applied, more stable foams can be generated, because the liquid recirculation enhances the liquid fraction of the foams. In wet foams coalescence and coarsening is reduced.

Besides the above-discussed design concepts, also other operational features play a role in the overall performance of a foam-bed photobioreactor. These include foam formation and foam break-up methods, which are essential elements for the operation of a foam-bed photobioreactor.

\subsubsection{Foam formation}

Regarding foam formation, surfactant selection was thoroughly studied in this thesis, however different foam generation methods were not investigated in detail. The gas distributor design has an influence on the bubble size distribution of the foams and also on the energy requirements for gas supply.

In order to create bubbles by gas sparging through an orifice, a certain pressure has to be applied. This pressure difference is described by $\Delta P=2 \cdot \gamma / r_{\text {orifice }}$, where $\gamma$ is the surface tension and $r_{\text {orifice }}$ is the orifice radius [11]. This equation shows that smaller orifices require higher pressures for bubble generation. The pressure requirement for bubble generation is $5800 \mathrm{~Pa}$ considering the lab-scale reactor applying a perforated plate with $15 \mu \mathrm{m}$ pore radius. In comparison, to overcome the hydrostatic pressure of a $2 \mathrm{~m}$ height foam column, only $590 \mathrm{~Pa}$ gas pressure is required. The power demand for gas compression to meet the pressure requirement for bubble generation has not been included in the model evaluation presented in Chapter 5 but clearly cannot be neglected. The gas distributor, however, has not been optimized 
yet. In case the orifice radius is increased to $100 \mu \mathrm{m}$, the pressure requirement for bubble generation can be reduced with a factor of 6.6 reaching a pressure drop of $870 \mathrm{~Pa}$.

A gas distributor plate with increased hole sizes is beneficial in terms of energy reduction for gas supply. Increasing orifice diameter would result in increasing bubble radius [11], leading to decreased liquid fraction of the foam, and therefore to suboptimal reactor operation conditions. Increased orifice size could be counterbalanced by the application of wetting orifices [11]. Wetting orifices have low contact angles with water, and their contact with the created air bubbles is minimal. This results in more confined gas streams and therefore smaller bubbles. Next to the wetting properties of the orifice, also the gas flow rates influence bubble sizes: higher gas flows result in bigger bubbles [11].

In the case of bubbling via several orifices (e.g. perforated plates) the bubble formation mechanism is complex since the pressure in the gas reservoir fluctuates and the gas bubbles also interact via hydrodynamic forces in the liquid phase. Therefore, the spacing and organization of the orifices also becomes dominant, which, together with the gas flow rate determine the bubble sizes and also the number of open orifices [11]. In contrast to perforated plates with uniform hole sizes, porous gas distributors own a diverse range of pore sizes. These gas distributors are more common and less costly. Porous distributors, however, create foams with polydisperse bubble size distribution, which might be prone to coalescence. Future studies therefore should focus on optimized and cost-effective gas distribution for foam formation.

\subsubsection{Foam breaking}

An ideal foam breaker for the foam-bed photobioreactor does not contaminate the media and also does not require energy for its operation. Therefore, antifoaming agents and mechanical foam breakers are not preferred techniques. In this thesis, two promising techniques for foam breaking were tested. These include foam breaking by solid hydrophobic materials and foam breaking by liquid jets falling on the foam. Although the liquid recirculation is an energy requiring process, its application is required for ensuring high microalgae content in the foam. Therefore, using the recirculated microalgal suspension also as liquid jets for foam breaking to the foam does not require additional energy.

The foam-breaking efficiency was not optimal in either of the foam-breaking methods tested. In the first design with an external foam breaker, the unbroken bubbles were returned to the reactor. Inefficient foam breaking in this reactor design might lead to $\mathrm{CO}_{2}$ depletion or $\mathrm{O}_{2}$ 
accumulation in the foam bubbles. In the second design applying internal foam disruption with liquid jets, inefficient foam breakage would result in overflowing foam exiting the reactor. To prevent the loss of microalgal culture contained within the unbroken foam, an additional foam level control was introduced. This was based on discontinuous gas supply: the gas flow was stopped in case the foam level was too high. The time period without gas supply allowed the bubbles that remained intact to collapse spontaneously. Although this strategy was efficient for foam volume control, temporarily stopping the $\mathrm{CO}_{2}$ supply introduced $\mathrm{pH}$ fluctuations, resulting in suboptimal growth conditions. Therefore, in future studies, an improved foam volume control has to be developed which does not require discontinuous gas supply.

Foam breaking by the liquid jets can be further optimized by re-designing the top of the foam column. Stronger liquid jets can be achieved when the diameter of the liquid recirculation inlet tubes are reduced. However, too high jet velocities might result in shear stress for the microalgal cells and both aspects need to be balanced. When designing the reactor top, the liquid jets should be prevented to penetrate deep in the column: only the top foam layer needs to be destroyed.

Generally, more efficient foam breaking can be achieved when the foam stability is reduced, which can be achieved by reduced surfactant concentration, reduced liquid recirculation rate and reduced gas flow rate. When this is not enough, and foam breaking is still inefficient, a combination of different foam breaking techniques needs to be used. Ultrasonic foam-breaking or mechanical foam breakers can also be used for foam volume control. Foam deconstruction can be induced by acoustic waves at a frequency of 20-30 kHz. Short ultrasonic pulses in the order of milliseconds can already cause defoaming $[12,13]$. Ultrasound in the same frequency range might affect the viability of microalgal cells under long-term exposure in the order of minutes [14]. Therefore, the intensity and exposure time has to be studied in order to determine whether ultrasonic foam breaking is possible without affecting cell integrity. Mechanical foam breakers, such as rotating disks or brush or comb type blades might also be applied [12], however, their application in a flat panel shaped foam-bed photobioreactor might be inconvenient and energy-intensive.

The energy demand of ultrasonic and mechanical foam breaking seem to be relatively high suggesting that the most promising and energy-efficient technology is the combination of hydrophobic solids and liquid jets. The bubbles that rise above the height of liquid recirculation jets can be further destabilized on the top of the reactor with a rough, hydrophobic reactor top. Rough or sharp surfaces might further facilitate the penetration of the foam-breaking materials 
to the liquid layer surrounding bubbles. For example, the application of needles can facilitate foam break-up [15].

\subsection{Surfactants}

The surfactant applied for foam stabilization in the foam-bed photobioreactor has a great influence on the foam formation and breakup processes. Amongst 10 surfactants tested, the non-ionic surfactant Pluronic F68 showed the best properties in terms of low toxicity, low biodegradability, and good foaming properties. The drawback of the application of non-ionic surfactants, and therefore also of Pluronic F68 is the low microalgae partitioning towards the foam phase. This can be circumvented by incorporating a liquid recirculation in the design, which also ensures equal microalgae distribution in the reactor and a more wet and stable foam. Because of the recirculation the liquid phase in the reactor will be well mixed and the algae concentration will be equal in the foam phase and liquid layer underneath foam. Therefore, for future experiments related to surfactant selection for foam-bed photobioreactors, microalgae partitioning becomes irrelevant and should not be considered.

\subsubsection{Challenges of Pluronic F68}

Cultivation of C. sorokiniana in Pluronic F68 stabilized foams revealed that the foam stability and the liquid fraction of the foam declined in time during batch operation. Simultaneously, the concentration of Pluronic F68 was also decreasing (See Chapter 3, Appendix 3.E). Possibly the surfactant molecules are absorbed to specific reactor surfaces, or the surfactant is sensitive to physical/chemical degradation. Surfactant loss or degradation would lead to increased surfactant requirements, increasing the operational costs of the foam-bed photobioreactor. Therefore, it is essential to reveal the mechanisms responsible for the observed decline of the surfactant concentration.

Preliminary experiments have shown that foam stability and liquid fraction were not influenced in the presence of $8.4 \mathrm{~g} \mathrm{~L}^{-1}$ C. sorokiniana dry weight concentration. This suggests that foam stability is not compromised in the presence of high biomass concentrations. Our results are in line with the findings of M. Vazquez, who demonstrated that the concentration of Pluronic F68 is not affected by high biomass concentrations. Therefore, the decrease of foaming properties in time are not reasoned by surfactant absorption to the microalgal cells. In contrast, thermal degradation of Pluronic F68 was reported by B. Erlandsson. The degradation was observed at 
a temperature of $40{ }^{\circ} \mathrm{C}$ over a period of several months [16]. This was confirmed by $\mathrm{M}$. Vazquez, who observed short-term degradation at $37{ }^{\circ} \mathrm{C}$ [M. Vazquez, personal communication], which is the optimal temperature of C. sorokiniana [17]. As shown in our experiments microalgae cultivation is still possible at $37{ }^{\circ} \mathrm{C}$ in foams stabilized by Pluronic F68. Nevertheless, it could be argued that because of thermal degradation more surfactant is required at $37^{\circ} \mathrm{C}$ than what would be needed at lower temperatures. In this context it should be noted that most microalgae have a lower optimal temperature than Chlorella sorokiniana [18].

In case of strains with a high temperature optimum, a surfactant other than Pluronic F68 might be more feasible. Surfactant selection should be focused on non-ionic surfactants with low toxicity. Natural surfactants and biosurfactants are generally not toxic [19]. Even some microalgal strains have the natural ability of foam production [20]. Algal surfactants are produced by healthy, exponentially growing cells, and the amount produced is dependent on the age of the culture, cell density and algal species [20]. It was also demonstrated that algal surfactants can generate stable foams with high microalgae content [21]. An example of a foam stabilized by algal substances is presented in Figure 6.2. Algal surfactants could contribute to a self-sustaining reactor, however, their biodegradability might limit their application and has to be studied.

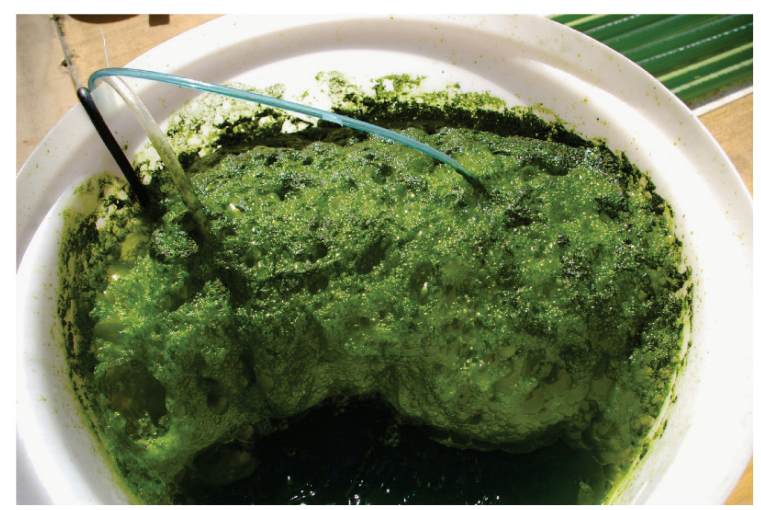

Figure 6.2. Foam with high microalgae content, stabilized by substances originating from a Neochloris spp. culture (photograph Niels-Henrik Norsker).

\subsubsection{Sensitivity to salinity}

The ideal surfactant for the foam-bed photobioreactor should be applicable under different conditions. Due to increasing freshwater scarcity, algae production with non-potable water is desired, and therefore seawater or wastewater are preferred [22]. However, the high electrolyte 
concentration of seawater might affect the foam stability. It was shown that different ions can have adverse effect on the stability of foams formed by non-ionic surfactants [23, 24]. Therefore, we performed preliminary experiments to evaluate the effect of seawater on the stability of foam formed by Pluronic F68. The artificial seawater employed contained additional sodium chloride $(\mathrm{NaCl})$ compared to freshwater medium [25], which resulted in an increase of ionic strength of $450 \mathrm{mM}$. In Figure 6.3 it can be seen that the bubble size, liquid fraction and stability of foams stabilized by Pluronic F68 were not influenced by the increased ionic strength of seawater medium. Pluronic F68 therefore offers the possibility of microalgae cultivation under saline conditions, broadening the applicability of foam-bed photobioreactors to marine algal strains.
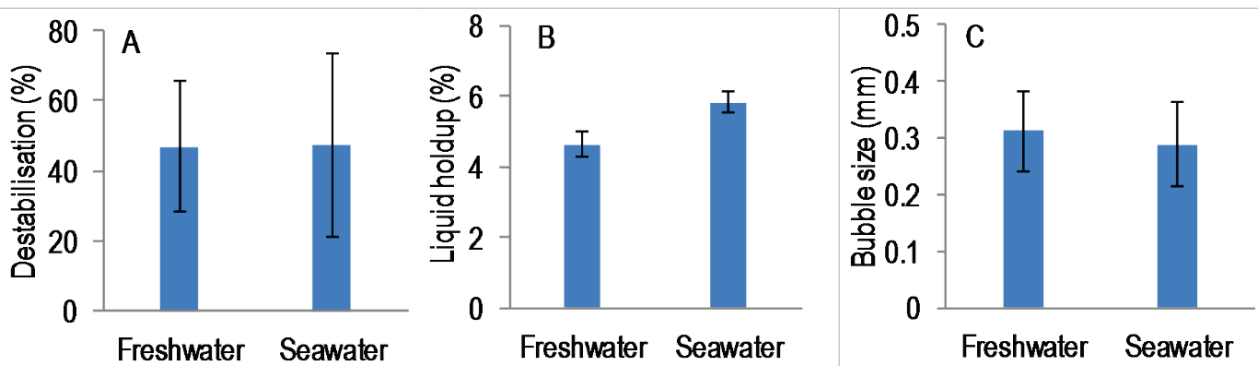

Figure 6.3. A) Foam stability expressed as foam volume destabilization in 1 hour B) liquid fraction of the foam C) average bubble size in the presence of fresh and seawater culture media. Experiments were carried out in the Foamscan (Teclis- IT Concept, Logessaigne, France), at 1 CMC Pluronic F68 concentration. The freshwater medium used was $3 x$ concentrated $\mathrm{M} 8 \mathrm{a}$ [9], while the seawater medium was adapted from [25] and contained in $\mathrm{g}$ $\mathrm{L}^{-1}: \mathrm{NaCl} 26 ; \mathrm{KNO}_{3} 3.4 ; \mathrm{Na}_{2} \mathrm{SO}_{4}$ 0.5; $\mathrm{MgSO}_{4} \cdot 7 \mathrm{H}_{2} \mathrm{O}$ 0.73; $\mathrm{CaCl}_{2} \cdot 2 \mathrm{H}_{2} \mathrm{O} 0.36 ; \mathrm{K}_{2} \mathrm{HPO}_{4} 0.43 ; \mathrm{NaHCO}_{3}$ 0.84; NaFeEDTA 0.01; and the following in $\mathrm{mg} \mathrm{L}^{-1}: \mathrm{Na}_{2}-\mathrm{EDTA} \cdot 2 \mathrm{H}_{2} \mathrm{O} 29.8, \mathrm{MnCl}_{2} \cdot 4 \mathrm{H}_{2} \mathrm{O} 3.8, \mathrm{ZnSO}_{4} \cdot 7 \mathrm{H}_{2} \mathrm{O} 1.2, \mathrm{CoCl}_{2} \cdot 6 \mathrm{H}_{2} \mathrm{O} 0.28$, $\mathrm{CuSO}_{4} \cdot 5 \mathrm{H}_{2} \mathrm{O} 0.33, \mathrm{Na}_{2} \mathrm{MoO}_{4} \cdot 2 \mathrm{H}_{2} \mathrm{O}$ 0.025; Biotin 0.025, vitamin B1 1.1, vitamin B12 0.135. The pH was adjusted with $\mathrm{NaOH}$ to $\mathrm{pH} 7.5$.

\subsubsection{Surfactant re-use}

The price of Pluronic F68 can be as low as 2.5 \$ per kilogram. When a Pluronic F68 concentration of $0.5 \mathrm{CMC}$ is used, the approximate price of surfactant per kilogram of algal biomass produced is 1.2 eurocents. In comparison, the operational energy requirements including gas supply, liquid recirculation and harvesting adds up to 0.8 eurocents per kilogram of biomass produced. This was calculated by converting the biomass specific energy requirement of a $5 \mathrm{~cm}$ deep foam-bed photobioreactor, $0.23 \mathrm{~kJ} \mathrm{~g}^{-1}$ to cost by employing an energy cost price of $0.122 € \mathrm{kWh}^{-1}$ [6]. Although both cost factors are low compared to a cost of $3.4 € \mathrm{~kg}^{-1}$ calculated for a 'traditional' flat panel photobioreactor [6] surfactant costs are 1.5 
times higher compared to the operational costs. A further reduction in cost price can be achieved when the surfactant is reused.

Next to reduced costs the re-use of surfactants in general is a more environmental friendly solution. It has been found that, the surfactant molecules do not absorb to microalgal cells and remain in the liquid phase after cell separation by centrifugation [M. Vazquez, personal communication]. Consequently, surfactants could be re-used by recycling the complete cultivation medium after biomass separation. Medium recycling also offers the advantage of reduced water and nutrient demand [26]. In some cases microalgal growth in recycled medium is hindered because of the accumulation of inhibitory compounds [27]. But, according to other studies, the productivity of $C$. vulgaris and C. sorokiniana is not affected by medium recycling $[28,29]$. Therefore, if medium recycling is possible with the cultivated algal strain, surfactant re-use by medium recycling is very attractive. By assuming that after centrifugation, a final biomass concentration of $220 \mathrm{~g} \mathrm{~L}^{-1}$ is achieved [30], approximately $92 \%$ of the surfactant molecules can be recycled ( $8 \%$ remains in the wet paste), resulting in less than 0.2 cents per $\mathrm{kg}$ of biomass produced.

In the situation the recycled media has an inhibitory effect on the growth, medium recycling is not a feasible option for surfactant recovery. This would require the specific removal of the inhibitory compounds while leaving the surfactant and inorganic matter in the recycled solution, which is not trivial. Instead, the surfactant molecules could be collected by foam-fractionation $[31,32]$ and thus making use of the surface active characteristic of surfactants. Although this would require an additional processing step and energy, the resulting concentrated surfactant stream could be re-used for foam formation.

The surfactant content of the final, dried biomass is expected to be as low as $0.04 \% \mathrm{w} / \mathrm{w}$ based on the water content of the algal paste remaining after centrifugation. Further studies are required to determine whether the residual Pluronic F68 molecules would limit the application of the biomass grown in a foam-bed in the food and feed industry. Pluronic F68 is approved by the Food and Drug Administration (FDA) as a wetting agent and wash solution for fruits and vegetables, or as an additive to materials in contact with food [33]. Additionally, Pluronic F68 is approved by FDA for intravenous injection [34]. 


\subsection{Guidelines operation foam-bed photobioreactor}

Several practical limitations in our lab-scale foam-bed photobioreactor restricted the experimentally achieved productivity. These include inaccurate foam level control, fluctuating $\mathrm{pH}$ values, biofilm formation, sub-optimal dilution rate and liquid recirculation rates, and high dark culture volume. The $\mathrm{pH}$ fluctuations can be reduced by improved foam breaking leading to continuous gas supply. Nevertheless, an active $\mathrm{pH}$ control should still be introduced. Additionally, the nutrient concentration in the growth medium should be increased to enable growth to even higher biomass concentrations. Elevated biomass concentrations allow for a reduced energy requirement for harvesting. In addition, liquid recirculation rates can be decreased resulting in a reduction of the operational energy required. When $60 \mathrm{~g} \mathrm{~L}^{-1}$ biomass concentration could be achieved in the foam-bed photobioreactor, the productivity would increase and the operational energy requirements per gram of biomass produced would decrease by more than a factor of two (Chapter 5). The feasibility of a growth medium supporting such high biomass concentrations was already demonstrated during heterotrophic cultivation of microalgae, where biomass densities above $100 \mathrm{~g} \mathrm{~L}^{-1}$ were achieved [35].

In order to achieve maximal reactor productivity, the dark culture volume in the reactor has to be minimalized. Dark parts of the reactor include the liquid recirculation line and the liquid layer underneath the foam column. The dark culture volume present in the liquid recirculation tubes can be reduced by applying tubes with small diameter. Besides, the liquid layer on the bottom of the reactor has to be minimal. Above the gas distributor, a few centimetres of liquid layer has to be present to ensure continuous foam generation and liquid recirculation, resulting in notable dark liquid volume when the gas distributor is placed over the whole cross section. Therefore, gas distributors over only a reduced fraction of the cross section might be beneficial. These thin gas distributors has to be surrounded by steep reactor walls to avoid settling of algal cells on horizontal surfaces, as illustrated in Figure 6.4. 


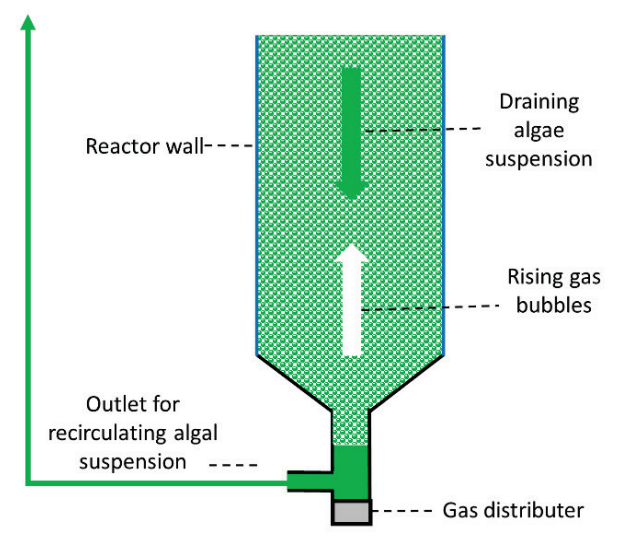

Figure 6.4. Conceptual reactor bottom for minimizing the non-illuminated culture volume.

The dilution rate and liquid recirculation rate are key operational factors for the foam-bed photobioreactor, determining its productivity and energy requirements. In our experiments the liquid recirculation rate applied was an order of magnitude higher compared to the optimal values determined in the model calculations. According to the model calculations, reduced liquid recirculation rates are expected to lead to an increase of the productivity and a decrease of the operational energy requirement. In order to evaluate the effect of reduced liquid recirculation rates on foam stability, foam-breaking efficiency and mixing properties, further lab-scale experiments are necessary. During our experiments with reduced liquid recirculation rates, the liquid distribution via the liquid inlet tubes was inhomogeneous. This problem can be circumvented by applying inlet tubes with reduced diameter ensuring equal flow, which also contributes to increased foam breaking efficiency. Reduced liquid recirculation rates also result in reduced liquid fractions and, consequently, reduced foam stability. The desired foam stability possibly can be restored with higher surfactant concentrations. Mixing in the reactor still remains a challenge, and therefore dissolved $\mathrm{O}_{2}, \mathrm{CO}_{2}$, temperature and $\mathrm{pH}$ gradients might take place. The mixing properties, including the extent of these gradients should be experimentally determined in order to assess the possibility of growth reduction due to sub-optimal cultivation conditions. When these practical challenges are solved, the predicted low energy requirements for microalgal production in foam-bed photobioreactors can be met in practice. 


\subsection{Outdoor application foam-bed photobioreactor}

Industrial cultivation of microalgae for the production of food, biofuels and chemicals requires large-scale outdoor reactors. Larger reactor modules lead to the reduction in initial investment costs of the reactor construction, since an increased amount of biomass can be produced within one single unit. Regarding the design of outdoor large scale reactors, several relevant issues will arise. These include foam generation and breakage, accurate $\mathrm{pH}$ and temperature control, equal liquid and gas distribution over the whole cross section of the reactor, material choice of large scale reactors, and positioning of the reactor modules. The greatest challenge foreseen in scaling up foam-bed photobioreactors is related to temperature control, which will be discussed in more detail.

\subsubsection{Scale-up principles}

The most important aspect of a large-scale foam-bed photobioreactor is related to its dimensions and design. Rectangular reactors, such as flat panels are suggested for scale-up purposes instead of cylindrical shape due to their easy scalability. A convenient way of scaling foam-bed photobioreactors is increasing their width and height. Reactor width can be increased without any influence on the operation. However, with increasing reactor height, coalescence of the foam bubbles will become more dominant. During the elongated time periods that bubbles rise, the pressure difference between adjacent bubbles increases and disproportionation takes place. Therefore, higher reactors will require increased surfactant concentrations while foam destabilization during rising to higher levels will facilitate foam breakage. Additionally, with higher reactors and given $\mathrm{CO}_{2}$ concentrations, the superficial gas flow velocity has to be increased to provide sufficient $\mathrm{CO}_{2}$ for algal growth.

For energy-efficient operation of outdoor reactors, the liquid recirculation rate should be adjusted according to the solar irradiation. On cloudy days with low irradiance, the liquid recirculation rate could be reduced to ensure an optimal light profile, while on sunny days higher recirculation rates could increase the productivity. By applying a reduced liquid recirculation rate on cloudy days, a reduced liquid fraction and increased light penetration can be achieved to compensate for the lower light levels. Similarly, during the night, reduced gas flow rates and surfactant concentrations could be applied to facilitate foam break-up with reduced or completely without liquid recirculation. The absence of liquid recirculation during the night would lead to significant energy saving. 
The foam-bed reactor modules, similar to large-scale flat panels, should be placed in parallel rows [36]. Parallel reactors can reduce light losses by reflection, because the reflected light from one panel can be absorbed by another reactor. Reactor orientation, spacing of the reactors and reactor height should be modelled an optimized in order to achieve maximal productivity of a panel. Tilting of the reactor surface for maximal perception of solar irradiance is not advantageous because the liquid would drain down along the back wall of the reactor leading to inhomogeneous liquid fraction distribution across the reactor depth. Light dilution by closely spaced reactors might be advantageous to eliminate photoinhibition and overheating during mid-day sunny hours. Flat panel foam-bed photobioreactors orientating east-west [8] might also contribute to reduced overheating at solar noon, where the maximal solar irradiation takes place.

We learned from preliminary experiments that the material of the reactor wall has a direct influence on foam stability. Hydrophobic materials with large contact angles own a defoaming capacity, which might limit their application. The defoaming capacity of hydrophobic particles due to dewetting was reported earlier [23]. Optimally, an inexpensive plastic bag could be applied as a support for the foam. The low pressure of the foam column allows for the application of thin plastic films. For example low-density polyethylene could be applied, since it was used successfully for the construction of the Green Wall Panel photobioreactor design [37] and the Proviron photobioreactor concept [38]. However, plastics usually own a hydrophobic character, described by large contact angles [39]. When these materials are in contact with the liquid film surrounding the bubbles, the surface tension of the liquid in contact with the solid material is reduced which leads to foam collapse [40]. For applications as a support material, different plastic materials should be tested for their contact angles and defoaming capacities. Surface modification of plastic polymers might also be applied to render hydrophobicity [41]. However, the limited duration of these surface modifications which is in the range of hours or days [42, 43] might restrict their application. Alternatively, glass walls could be used because its hydrophilic nature and its low contact angle after a sulfuric acid peroxide treatment. Glass walls would increase the capital costs of foam-bed photobioreactors, however, their lifetime is longer than those of plastics. Contact angles of different materials are given in Table 6.1. However, it has to be noted that the foam breaking capacity of materials is related to the reduction of the surface tension of the surfactant-containing algal solution in contact with the given materials. This reduction in surface tension is related to, but not exactly reflected by the contact angles. 
Table 6.1. Contact angles of different materials.

\begin{tabular}{lcc}
\hline Material & Contact angle & Reference \\
\hline Glass & $51^{\circ}$ & {$[44]$} \\
Glass treated with $\mathrm{H}_{2} \mathrm{SO}_{4}-\mathrm{H}_{2} \mathrm{O}_{2}$ mix & $12^{\circ}$ & {$[45]$} \\
Polyethylene (PE) & $95^{\circ}$ & {$[39]$} \\
Poly(methyl methacrylate) (PMMA) & $65^{\circ}$ & {$[42]$} \\
Polyvinyl chloride (PVC) & $85^{\circ}$ & {$[46]$} \\
Foam breaking materials (PTFE\&PDMS) & $100-116^{\circ}$ & {$[47,48]$} \\
Oxygen plasma treated PMMA & $<5^{\circ}$ & {$[42]$} \\
Oxygen plasma treated PE & $35^{\circ}$ & {$[49]$} \\
UV initiated surface graft polymerization of PE & $<5^{\circ}$ & {$[50]$} \\
\hline
\end{tabular}

\subsubsection{Temperature control}

Heat accumulation in outdoor photobioreactors is an important issue, since high temperatures will inhibit algal growth and even lead to cell death. In photobioreactors with reduced liquid volume, heat can accumulate quickly. Therefore, it is important to apply cooling to remove the accumulated heat from the foam-bed photobioreactor. The temperature increase in the photobioreactor can be calculated based on the sunlight irradiance and the natural heat exchange of the photobioreactor with the environment via convection and radiation [51]. The detailed description of the heat-exchange of the foam-bed photobioreactor is presented in Appendix 6.A. During these calculations, different environmental conditions were considered by varying the sunlight irradiation, wind speed and ambient temperature.

Our calculations show that without cooling, the temperature of the microalgal suspension increases rapidly: the temperature can rise in summer days to inhibiting values in the order of minutes. With a high wind speed of $6 \mathrm{~m} \mathrm{~s}^{-1}, 25^{\circ} \mathrm{C}$ ambient temperature, and a maximal daily irradiation of $900 \mathrm{~W} \mathrm{~m}^{-2}$, within 10 minutes the temperature of a $C$. sorokiniana culture will rise from 37 to $65^{\circ} \mathrm{C}$.

In order to provide sufficient cooling, a tubular heat exchanger could be integrated with the liquid recirculation line. In this case, the temperature of the microalgae solution drops while it is flowing through the recirculation tube and the temperature will increase again while the algae solution drains down in the foam-bed exposed to sunlight. Our results suggest that during the time the algal suspension drains down the foam column, the temperature increase of the suspension is already far beyond tolerable for the cells during solar noon $\left(900 \mathrm{~W} \mathrm{~m}^{-2}\right)$. In fact, 
at an average wind speed of $3 \mathrm{~m} \mathrm{~s}^{-1}$, and an ambient temperature of $30{ }^{\circ} \mathrm{C}$, the culture can only take $180 \mathrm{~W} \mathrm{~m}^{-2}$ before its temperature during drainage increases more than $8{ }^{\circ} \mathrm{C}$. Integrated cooling in the liquid recirculation line therefore is not a feasible solution to prevent overheating of the cultures.

In order to prevent overheating the whole surface area of the reactor must be used for cooling. For example, a cooling chamber could be introduced on one side of the reactor, in which cooled liquid is recirculated. This solution allows for continuous cooling, however, substantial energy requirements are involved when providing such a continuous water flow. In order to reduce the heating of the microalgal culture and the need for cooling liquid flow, near-infrared (NIR) filters could be applied $[51,52]$. These filters allow for PAR transmission, while they reflect the NIR radiation efficiently. NIR filters allow for 50\% reduction in incident solar energy, reducing the heat load by the same factor [52]. Integrating the photobioreactor within buildings also offers a solution for reduced temperature exchange needs because of the thermal exchanges between the building and the photobioreactor [53].

Alternatively, the reactor could be submerged in a large water volume [54]. The large water volumes has a buffering capacity preventing quick temperature changes. Therefore, for temperature regulations of the water pool, less energy requirements are expected. However, when the foam-bed photobioreactor is in contact with a water column of higher hydrostatic pressure, thicker plastic walls should be applied. In this case, the benefit of material cost reduction due to the low hydrostatic pressure of a foam-bed is lost, because the greater pressure of the water pool.

\subsection{Comparison foam-bed photobioreactors and advanced photobioreactor designs}

With the aim of achieving high biomass densities, several novel photobioreactor designs are currently under development (Figure 6.5). These include ultrathin flat-panels with a light path of $7.5 \mathrm{~mm}$ [55], 'rain' reactors based on falling droplets of algal suspension [56], biofilm photobioreactors [57], and thin-layer photobioreactors [58]. A comparison can be made between these photobioreactors in terms of biomass density, mass transfer, energy requirements for operation, and expected investment costs. 
Porous substrate biofilm reactors (PSBR) are advanced biofilm photobioreactors that are based on immobilized microalgae cultivation on porous substrates. Next to algal attachment, these porous substrates allow for the diffusion of water and nutrients to the biofilm at the nonilluminated surface. Compared to conventional biofilm reactor designs with a liquid layer between the gas and liquid phases, the PSBR biofilm is in direct contact with the gas phase, allowing for increased mass transfer, decreased cell detachment and higher light intensities. A great advantage of this system lies in the remarkably reduced liquid volumes applied and extremely high biomass densities achievable. Biomass densities between 150- $300 \mathrm{~g} \mathrm{~L}^{-1}$ can be achieved (Table 6.2), where the upper limit corresponds to the maximal value restricted by the water content of the cells. The biomass specific energy needs for the liquid pumps are estimated to be $3.6 \mathrm{KJ} \mathrm{g}^{-1}$ dry biomass [59], which is significantly higher compared to the predicted energy needs of the foam-bed of $0.23 \mathrm{KJ} \mathrm{g}^{-1}$. Mass transfer takes place via the surface of the biofilm which is either open to ambient air [59], or is in contact with $\mathrm{CO}_{2}$ enriched air supplied to a closed chamber [60]. When high growth rates are desired, $\mathrm{CO}_{2}$ supply above air concentration is required. Drawbacks of these reactor types are the energy required for liquid pumps and the high material costs for the porous substrates [57].

The thin-layer photobioreactors are based on a flowing liquid film over a tilted surface [58]. The thickness of the algal suspension in lab-scale reactors is in the range of $1.5-2 \mathrm{~mm}$, resulting in a specific surface area of $500 \mathrm{~m}^{-1}$. In outdoor systems the culture depth is increased to 6-8 $\mathrm{mm}$ [61]. $\mathrm{CO}_{2}$ can be supplied to a closed chamber above the liquid film [58], or it can be sparged within the suspension in the liquid reservoir at the bottom of the slope [61]. In reactors equipped with a closed gas chamber reduced gas flow rates can be applied as a result of high mass transfer rates, long gas residence time and uncoupled culture mixing from gas supply. The main operational energy requirement for thin-film photobioreactors is related to the continuous liquid recirculation from the bottom to the top of the slope. According to our calculations based on an outdoor thin layer photobioreactor [61], the biomass specific energy requirement for the liquid pumps is approximately $2.9 \mathrm{KJ} \mathrm{g}^{-1}$.

The rain reactor is based on spraying microalgal suspension on the top of several, stacked horizontal meshes. From the top of the reactor, the algae-containing liquid droplets are falling due to gravity. The meshes slow down the fall of droplets, thereby enhancing the residence time of the suspension in the reactor. Hanging droplets are formed on the meshes until the droplet grows big enough to fall due to gravity until the next mesh. The microalgal solution is circulated over the reactor by pumping the collected liquid from the bottom of the greenhouse to a liquid 
distributor above the meshes. Gas exchange takes place via the liquid droplets and the $\mathrm{CO}_{2}$ enriched air present in the greenhouse [56]. The theoretical advantage of high biomass densities and biomass productivities of these reactors is not yet achieved in practice (see Table 6.2). The low culture volumes, enhanced mass transfer and short light path in the small droplets are beneficial characteristics of these systems. However, the large reactor volume, high material requirement for the construction of the greenhouse and of the meshes, the pumping energy requirement and the shading introduced by the meshes are introducing major challenges.

Flat-panels with reduced culture depth are advantageous to reduce light path and achieve high biomass densities. The minimal depth of the photobioreactors is dictated by the size of the gas bubbles, generated for culture mixing and $\mathrm{CO}_{2}$ supply. A $7.5 \mathrm{~mm}$ deep photobioreactor was already reported for efficient microalgae production [55]. Thin flat-plate photobioreactors require vigorous mixing in order to achieve sufficient gas transfer capacity which also eliminates photo-damage by frequent light/dark cycling [62]. The gas flow rates applied are remarkably higher than the gas flow rates required for the liquid foam-bed, or the other reactors listed above. 

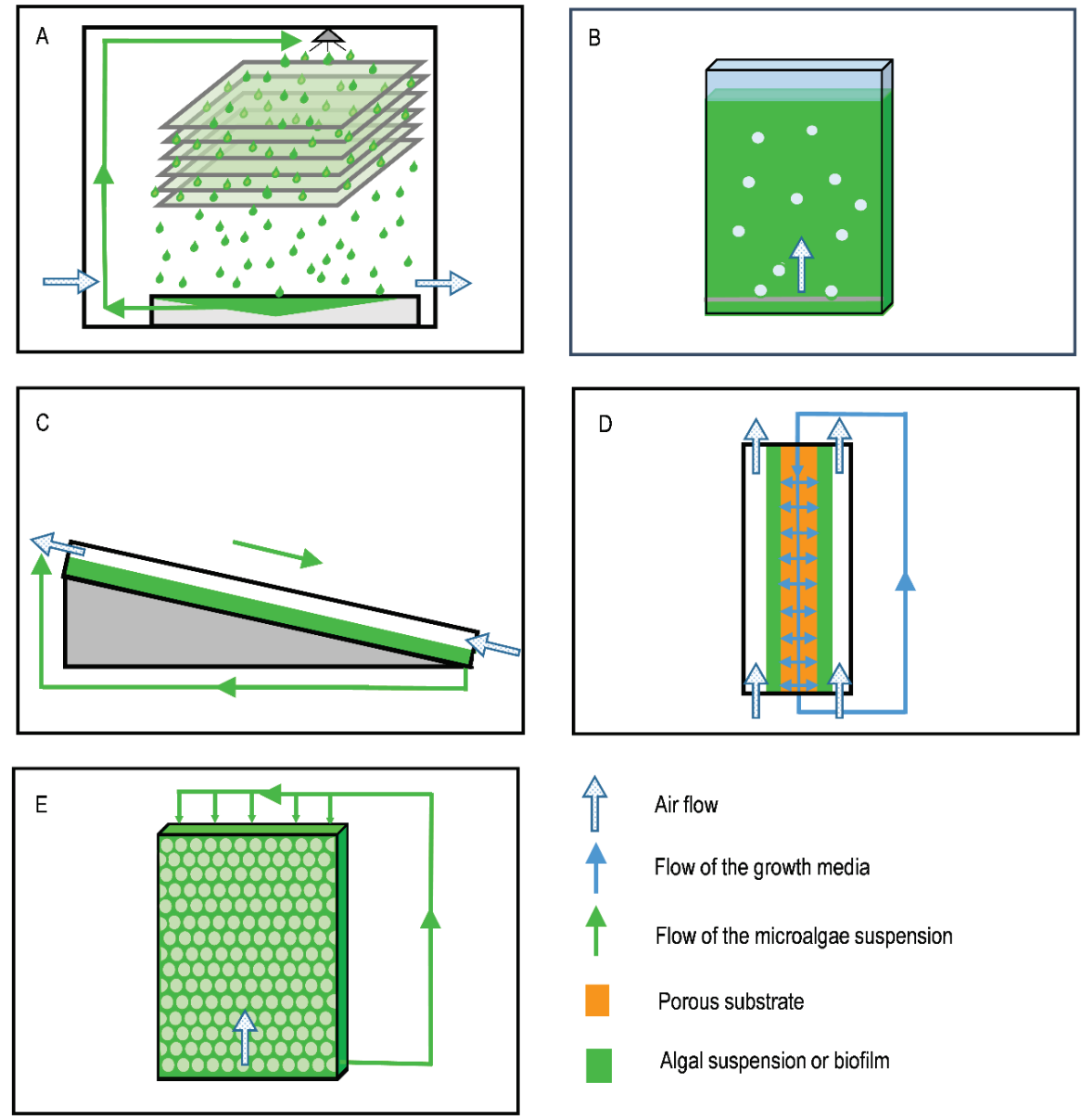

Algal suspension or biofilm

Figure 6.5. Scheme of the different, advanced photobioreactor designs. A) rain reactor, B) thin flat panel, C) thinlayer reactor, D) porous substrate biofilm reactor, E) foam-bed reactor. 
Table 6.2. Optimal biomass concentration expressed as gram dry weight per litre of harvested culture volume, areal productivity and biomass specific energy requirements of the different type of photobioreactors. The presented numbers only serve as an indication of the capacity of the systems, since different light conditions were applied.

\begin{tabular}{|c|c|c|c|c|c|c|c|}
\hline \multirow[b]{2}{*}{ Biomass conc. [g L-1] } & \multicolumn{2}{|c|}{ Rain reactor } & \multirow{2}{*}{$\begin{array}{c}\begin{array}{c}\text { Thin flat } \\
\text { panel }\end{array} \\
27^{c}\end{array}$} & \multirow{2}{*}{$\begin{array}{c}\text { Biofilm } \\
\text { PSBR } \\
150-300^{e}\end{array}$} & \multicolumn{2}{|c|}{ Thin Layer } & \multirow{2}{*}{$\frac{\text { Foam-bed }}{30-60}$} \\
\hline & $26^{a}$ & $8^{b}$ & & & $40-50 \mathrm{~g}$ & $24 i$ & \\
\hline Areal productivity $\left[\mathrm{g} \mathrm{m}^{-2} \mathrm{~h}^{-1}\right]$ & $3.0-6.1^{\mathrm{a}}$ & $0.3-1.0^{\mathrm{b}}$ & $4.6^{c}$ & $1.3^{e}$ & $1.6^{\mathrm{g}}$ & $0.9{ }^{i}$ & 2.8-3.1 \\
\hline Energy requirements [KJ g-1] & & . & $4.03^{c, d}$ & $0.07^{e, f-3.6^{f}}$ & $2.53^{g, h}$ & $53^{\mathrm{i}, \mathrm{h}}$ & $0.11-0.23$ \\
\hline
\end{tabular}

a model predictions for a sunny day disregarding light attenuation by the meshes [56]

${ }^{b}$ experimentally achieved data under $500-530 \mu \mathrm{mol} \mathrm{m}^{-2} \mathrm{~s}^{-1}[56]$

${ }^{c}$ continuous light of $900 \mu \mathrm{mol} \mathrm{m}^{-2} \mathrm{~s}^{-1}[55]$

${ }^{d}$ Calculated with 0.85 vvm gas flow rate. $0.85 \mathrm{vvm}$ was obtained by assuming the same volumetric gas flow rate demand reported for $2 \mathrm{~cm}$ deep reactors [6] and $0.75 \mathrm{~cm}$ reactors.

e Biofilm continuous light of $1023 \mu \mathrm{mol} \mathrm{m} \mathrm{m}^{-2} \mathrm{~s}^{-1}[63]$

${ }^{f}$ Calculated with a liquid flow rate of $6 \mathrm{~L}^{-1} \mathrm{~h}^{-1} \mathrm{~m}^{-1}$ reactor module [59]. Lower limit represents our calculations for the productivity reported in the table, while the upper limit is a reported value under outdoor, low light conditions of 18-320 $\mu \mathrm{mol} \mathrm{m}^{-2} \mathrm{~s}^{-1}$ at noon [59].

9 obtained in an outdoor reactor under fed-batch operation under $\sim 1200 \mu \mathrm{mol} \mathrm{m}^{-2} \mathrm{~s}^{-1}$ light intensity calculated for 15 hours daylight period (24 hours average of $800 \mu \mathrm{mol} \mathrm{m}^{-2} \mathrm{~s}^{-1}$ ) [64]

h Liquid flow rate is based on [61]

i obtained in an indoor photobioreactor $500 \mu \mathrm{mol} \mathrm{m}^{-2} \mathrm{~s}^{-1}$ as a daily average [58]

${ }^{\mathrm{i}}$ continuous light of $1500 \mu \mathrm{mol} \mathrm{m} \mathrm{m}^{-2} \mathrm{~s}^{-1}$ (model predictions of Chapter 5)

The maximally achieved microalgae concentration in the harvest is remarkably higher in the biofilm PSBR compared to all other photobioreactor types (Table 6.2). The biomass density of thin flat panels, thin layer photobioreactors and rain reactors are below the theoretical capacity of a foam-bed. This is because the foam-bed has a shorter, adjustable light absorption path, which is dictated by the liquid recirculation rate applied. The light path in the foam-bed can be as low as $0.8-1.3 \mathrm{~mm}$, which is reduced compared to the $7.5 \mathrm{~mm}$ presented for thin flat panels or $1.5-2 \mathrm{~mm}$ for thin layer photobioreactors. In terms of reactor areal productivity, thin flat panels, rain reactors and foam-beds are promising.

The reactor volume required for the production of a given amount of biomass is an indicator of the investment costs for cultivation facilities. Largest reactor volume is required for the rain reactor, followed by the foam-bed due to the high gas holdup. However, foam-beds own a low pressure and therefore the material costs for their construction can be reduced. Besides reactor volume, the increased material requirement in rain and porous substrate reactors also contribute to the initial costs.

Next to the investment costs, operational costs are determining the success of photobioreactors. PSBRs, foam-bed, thin-layer and the rain photobioreactors require liquid recirculation and 
therefore energy for the operation of liquid pumps. In contrast, thin flat-panel photobioreactors are the only type that do not require liquid pumping, but they do require high gas supply rates for mixing and mass transfer. In the other reactor types besides the flat panels the residence time of the supplied gas in the photobioreactor is high, and therefore sufficient mass transfer can be achieved at low gas supply rates. These reactors are based on entrapping the gas phase in a closed environment to reduce the need for gas supply. The gas phase can only be entrapped in the reactor when the mixing of the culture is independent on the gas flow rates. For mixing in these reactors the liquid phase is circulated instead. In terms of energy requirements for gas supply, thin-films and biofilms with closed gas chambers, foam-beds, and rain reactors require less energy because of the low gas flow rates applied.

The total energy requirement for gas supply, liquid recirculation and biomass separation is the lowest when biofilm reactors are applied (Table 6.2). However, harvesting large scale biofilm photobioreactors is challenging since the biofilms have to be scraped off regularly. In case of liquid phase based cultivation, including the foam-bed photobioreactor, the high density microalgal culture can be pumped out of the photobioreactor. Pumping the algal suspension offers an easier, automatized solution for harvesting the biomass. Although foam-beds require energy for the operation of both gas and liquid pumps, biomass production in these systems are still an order of magnitude less energy-intensive compared to thin-layers and thin flat panels.

The mass transfer in foam-beds is improved compared to biofilm, thin-film or flat panel systems because of a higher gas-liquid surface area in liquid foam-beds. Regarding biofilm and thin layer photobioreactors, the interfacial area available for mass transfer equals to the light illuminated surfaces. In contrast, foam-beds and rain reactors have larger inner surfaces and, consequently, improved gas transfer rates can be achieved.

One of the common features of all novel photobioreactor types lies in the reduced liquid volumes which allow for high-density microalgae cultivation. Reduction of culture volume has the advantage of a reduced water footprint and reduced requirements for operational energy and harvesting energy. Reduced culture volume stand in parallel with the need for effective cooling, and therefore all reactors with high surface to volume ratios will encounter the same challenge related to efficient and cost-effective temperature regulation during scale-up. Generally, these advanced photobioreactor designs have demonstrated that by applying reduced liquid volume and reduced light path, microalgae cultivation can become more efficient in terms of gas transfer, water demand and energy requirements compared to traditional systems. 


\subsection{Conclusions}

Foam-bed photobioreactors can lead to a major reduction of the energy requirement for algal biomass production. When our predictions in terms of productivity and biomass density for foam-beds will be reached in large-sale reactors, foam-beds will be cost-efficient algae production systems. Although improvements are still required with respect to reactor design and control strategies, the experimental results and model predictions demonstrated the benefits of this novel concept. Improvements of the foam-bed photobioreactor should focus on optimized foam formation, foam break up and temperature regulation. These improvements will lead to increased productivities, biomass densities and consequently to reduced biomass production costs. Considering economic feasibility next to energy, water, nutrient and $\mathrm{CO}_{2}$ use efficiency, the concept of a foam-bed photobioreactor represents a competitive alternative to other recent innovations in photobioreactor design. 


\section{Appendix 6.A}

In our calculations, the thermal model developed by Goetz et al. [51] was applied, which links the temperature of the microalgal suspension $\left(T_{s}\right)$ and the temperature of the glass reactor walls $\left(T_{g}\right)$ to sunlight irradiance, convective heat transfer and radiative heat transfer (Equation 6.A.1, 6.A.2 and 6.A.3). The heat transfer of a flat panel foam-bed photobioreactor was modelled which is illuminated by sunlight from one side. This model was further modified to include the evaporative heat transfer taking place in the foam bubbles. We assumed that the bottom and top side of the flat panel photobioreactor did not participate in heat transfer. The parameters applied in the model are presented in Table 6.A.1.

The sunlight radiance is composed of radiations in the ultravoilet (UV), photosynthetically active (PAR) and near infrared (NIR) ranges, accounting for $5 \%, 45 \%$ and $50 \%$ of the total radiation. Because the photosynthetic efficiency of a microalgae culture is around $5-13 \%$ of the PAR region [65], only a small fraction of sunlight irradiation is converted to biomass and more than $95 \%$ of the total absorbed light is converted to heat [53]. Therefore, in the thermal model all irradiation was assumed to contribute to heat production.

$\rho_{g} \cdot V_{g} \cdot c_{P}^{g} \frac{d T_{g}}{d t}=q \cdot A_{\text {ill }} \cdot \alpha_{g}-\varepsilon_{g} \cdot \sigma \cdot A_{\text {tot }} \cdot\left(T_{g}{ }^{4}-T_{\text {sky }}{ }^{4}\right)-h_{g / s} \cdot A_{\text {tot }} \cdot\left(T_{g}-T_{s}\right)-h_{g / a m b} \cdot A_{\text {tot }} \cdot\left(T_{g}-T_{\text {amb }}\right)$

Equation 6.A.1

$\rho_{s} \cdot V_{s} \cdot c_{P}^{S} \frac{d T_{s}}{d t}=q \cdot$ Aill $\cdot \alpha_{s} \cdot \tau_{g}-h_{g / s} \cdot A_{\text {tot }} \cdot\left(T_{g}-T_{s}\right)-h g_{\text {amb }} \cdot A_{\text {tot }} \cdot\left(T_{s}-T_{g}\right)+q^{\text {evap }} \quad$ Equation 6.A.2

$\begin{array}{ll}h /_{\text {amb }}=5.7+3.8 \cdot v & \text { Equation 6.A.3 }\end{array}$

The heat removed by evaporation of water $\left(q^{\text {evap }}\right)$ within the foam phase was calculated taking into account the superficial gas flow velocity $\left(F_{G}\right)$, and the inlet and outlet humidity $(R H)$. Based on the ambient temperature, the air saturation pressure $\left(P_{\text {sat }}\right)$ was determined (Equation 6.A.4).

$q^{\text {evap }}=\left(P_{\text {sat }}^{\text {in }} \cdot R H^{\text {in }}-P_{\text {sat }}^{\text {out }} \cdot R H^{\text {out }}\right) \cdot \frac{M D_{\text {air }}}{P_{\text {tot }}} \cdot M W_{\text {water }} \cdot F_{G} \cdot \Delta h^{\text {evap }}$

Equation 6.A.4

The sunlight emission $(q)$, wind speed $(v)$, and air temperatures $\left(T_{a m b}\right)$ are location dependent. When considering a location in Perpignan, southern France, the wind speed falls in the range of 0-6 $\mathrm{m} \mathrm{s}^{-1}$. The sunlight irradiation can reach up to $1000 \mathrm{~W} \mathrm{~m}^{-2}$ on a sunny summer day, while the maximum ambient temperature can be above $30{ }^{\circ} \mathrm{C}[51]$. Generally, vertical reactor panels receive less light illumination compared to the ground surface of the same area since the 
sunlight does not fall perpendicular on the reactor surface. However, it is possible that vertical panels of north-south orientation intercept $1000 \mathrm{~W} \mathrm{~m}^{-2}$ during noon hours [8].

The time required for the recirculated microalgae solution to drain down along the foam is defined by the residence time of the recirculated liquid in the foam column. When considering a superficial liquid recirculation rate of $0.052 \mathrm{~mm} \mathrm{~s}^{-1}$, and a $2 \mathrm{~m}$ high reactor filled with foam with a liquid fraction of $3 \%$, the residence time is 19 minutes. Therefore, the temperature increase of the microalgae suspension has to be determined for the 19 minutes time period.

Table 6.A.1. Parameters applied in the thermal model.

\begin{tabular}{|c|c|c|c|}
\hline Symbol & Description & Value & Unit \\
\hline$w$ & Reactor width & 1 & $\mathrm{M}$ \\
\hline$d$ & Reactor depth & 0.05 & M \\
\hline$h$ & Reactor height & 2 & M \\
\hline$\rho_{g}$ & Density glass & 2500 & $\mathrm{~kg} \mathrm{~m}^{-3}$ \\
\hline$\rho_{s}$ & Density solution & 1000 & $\mathrm{~kg} \mathrm{~m}^{-3}$ \\
\hline$V_{g}$ & Volume glass $\left(=A_{\text {tot }} \cdot 0.005\right)$ & 0.0213 & $\mathrm{~m}^{3}$ \\
\hline$V_{s}$ & Volume solution $(=\varepsilon \cdot w \cdot d)$ & 0.025 & $\mathrm{~m}^{3}$ \\
\hline$C_{p}{ }^{g}$ & Specific heat capacity glass & 720 & $\mathrm{~J} \mathrm{~kg}^{-1} \mathrm{~K}^{-1}$ \\
\hline$C_{p} s$ & Specific heat capacity solution & 4180 & $\mathrm{~J} \mathrm{~kg}^{-1} \mathrm{~K}^{-1}$ \\
\hline$T_{a m b}$ & Ambient temperature $\left(25^{\circ} \mathrm{C}\right)$ & 298.15 & K \\
\hline$t$ & Time & 1154 & s \\
\hline$\varepsilon_{g}$ & Emissivity & 0.92 & - \\
\hline$\sigma$ & Boltzmann constant & $5.6703 \cdot 10^{-8}$ & $W m^{-2} K^{-4}$ \\
\hline$A_{\text {ill }}$ & Illuminated area $(w \cdot d)$ & 2 & $\mathrm{~m}^{2}$ \\
\hline$A_{\text {tot }}$ & Total surface area $(=2 \cdot w \cdot d+2 \cdot d \cdot h+w \cdot d)$ & 2 & $\mathrm{~m}^{2}$ \\
\hline$h_{g / s}$ & Convection heat exchange coefficient & 290 & $W \mathrm{~m}^{-2} \mathrm{~K}^{-1}$ \\
\hline$a_{g}$ & Absorptivity glass & 0.05 & - \\
\hline$a_{s}$ & Absorptivity solution & 1 & - \\
\hline$T_{g}$ & Transmittivity glass & 0.9 & - \\
\hline$T_{\text {sky }}$ & Sky temperature $\left(=0.0552 \cdot T_{\text {amb }}{ }^{1.5}\right)$ & 284.18 & K \\
\hline$T_{\max }$ & Maximal temperature allowed $\left(40^{\circ} \mathrm{C}\right)$ & 313.15 & K \\
\hline$\varepsilon$ & Liquid fraction & 0.025 & - \\
\hline$P_{\text {tot }}$ & Atmospheric pressure & 101325 & $\mathrm{~Pa}$ \\
\hline$P_{\text {sat }}^{\text {in }}$ & Saturation pressure at $T_{\text {amb }}$ & 3169.9 & $\mathrm{~Pa}$ \\
\hline$P_{\text {sat }}{ }^{\text {out }}$ & Saturation pressure at $T_{\max }$ & 7384.9 & $\mathrm{~Pa}$ \\
\hline$R H^{\text {in }}$ & Relatiove humidity in the ingoing gas & 60 & $\%$ \\
\hline RHout & Relatiove humidity in the outgoing gas & 100 & $\%$ \\
\hline$M D_{\text {air }}$ & Molar density of air at $37^{\circ} \mathrm{C}$ & 39.3 & $\mathrm{~mol} \mathrm{~m}{ }^{-3}$ \\
\hline$M W_{\text {water }}$ & Molar weight water & 18 & $\mathrm{~g} \mathrm{~mol}^{-1}$ \\
\hline$J_{g}$ & Superficial gas velocity & $510^{-4}$ & $\mathrm{~m} \mathrm{~s}-1$ \\
\hline$F_{g}$ & Volumetric gas flow rate & $2.510-5$ & $m^{3} s^{-1}$ \\
\hline$\Delta h^{\text {evap }}$ & Evaporation heat at $40^{\circ} \mathrm{C}$ & $\begin{array}{l}2405.87 \\
{[66]}\end{array}$ & $\mathrm{J} \mathrm{g}^{-1}$ \\
\hline
\end{tabular}




\section{References}

[1] C.J. Hulatt, D.N. Thomas, Productivity, carbon dioxide uptake and net energy return of microalgal bubble column photobioreactors, Bioresour. Technol. 102 (2011) 5775-5787.

[2] D.C. Perry, P. Stevenson, Gas absorption and reaction in a wet pneumatic foam, Chem. Eng. Sci. 126 (2015) 177-185.

[3] A. Schumpe, W.D. Deckwer, Gas holdups, specific interfacial areas, and mass transfer coefficients of aerated carboxymethyl cellulose solutions in a bubble column, Ind. Eng. Chem. Process Des. Dev. 21 (1982) 706-711.

[4] M. Jamnongwong, K. Loubiere, N. Dietrich, G. Hébrard, Experimental study of oxygen diffusion coefficients in clean water containing salt, glucose or surfactant: Consequences on the liquid-side mass transfer coefficients, Chem. Eng. J. 165 (2010) 758-768.

[5] J. Vasconcelos, J. Rodrigues, S. Orvalho, S. Alves, R. Mendes, A. Reis, Effect of contaminants on mass transfer coefficients in bubble column and airlift contactors, Chem. Eng. Sci. 58 (2003) 1431-1440.

[6] J. Ruiz, G. Olivieri, J. de Vree, R. Bosma, P. Willems, H. Reith, M. Eppink, D.M. Kleinegris, R.H. Wijffels, M. Barbosa, Towards industrial products from microalgae, Energy Environ. Sci. 9 (2016) 3036-3043.

[7] N.-H. Norsker, M.J. Barbosa, M.H. Vermuë, R.H. Wijfels, Microalgal production - A close look at the economics, Biotechnol. Adv. 29 (2011) 24-27.

[8] E. Sierra, F.G. Acién, J.M. Fernández, J.L. García, C. González, E. Molina, Characterization of a flat plate photobioreactor for the production of microalgae, Chem. Eng. J. 138 (2008) 136-147.

[9] A. Janoska, P.P. Lamers, A. Hamhuis, Y. van Eimeren, R.H. Wijffels, M. Janssen, A liquid foam-bed photobioreactor for microalgae production, Chem. Eng. J. 313 (2017) 1206-1214.

[10] P. Stevenson, X. Li, G.M. Evans, A mechanism for internal reflux in foam fractionation, Biochem. Eng. J. 39 (2008) 590-593.

[11] W. Drenckhan, A. Saint-Jalmes, The science of foaming, Adv. Colloid Interface Sci. 222 (2015) 228-259.

[12] M. Barigou, Foam rupture by mechanical and vibrational methods, Chem. Eng. Technol. 24 (2001) 659-663.

[13] G. Rodríguez, E. Riera, J.A. Gallego-Juárez, V.M. Acosta, A. Pinto, I. Martínez, A. Blanco, Experimental study of defoaming by air-borne power ultrasonic technology, Phys. Procedia 3 (2010) 135-139.

[14] E.M. Joyce, X. Wu, T.J. Mason, Effect of ultrasonic frequency and power on algae suspensions, J. Environ. Sci. Health, Part A: Toxic/Hazard. Subst. Environ. Eng. 45 (2010) 863-866.

[15] N. Deshpande, M. Barigou, Mechanical suppression of the dynamic foam head in bubble column reactors, Chem. Eng. Process.: Process Intensification 39 (2000) 207-217.

[16] B. Erlandsson, Stability-indicating changes in poloxamers: the degradation of ethylene oxide-propylene oxide block copolymers at 25 and $40^{\circ} \mathrm{C}$, Polym. Degrad. Stab. 78 (2002) 571-575.

[17] C. Sorokin, J. Myers, A high-temperature strain of Chlorella, Science 117 (1953) 330-331.

[18] M. Ras, J.-P. Steyer, O. Bernard, Temperature effect on microalgae: a crucial factor for outdoor production, Rev. Environ. Sci. Bio/Technol. 12 (2013) 153-164.

[19] M. Pacwa-Płociniczak, G.A. Płaza, Z. Piotrowska-Seget, S.S. Cameotra, Environmental applications of biosurfactants: recent advances, Int. J. Mol. Sci. 12 (2011) 633-654.

[20] V. Żutić, B. Ćosović, E. Marčenko, N. Bihari, F. Kršinić, Surfactant production by marine phytoplankton, Mar. Chem. 10 (1981) 505-520.

[21] A. Csordas, J.-K. Wang, An integrated photobioreactor and foam fractionation unit for the growth and harvest of Chaetoceros spp. in open systems, Aquacult. Eng. 30 (2004) 15-30.

[22] P.K. Campbell, T. Beer, D. Batten, Life cycle assessment of biodiesel production from microalgae in ponds, Bioresour. Technol. 102 (2011) 50-56.

[23] R. Pugh, Foaming, foam films, antifoaming and defoaming, Adv. Colloid Interface Sci. 64 (1996) 67-142.

[24] S.I. Karakashev, E.D. Manev, Frothing behavior of nonionic surfactant solutions in the presence of organic and inorganic electrolytes, J. Colloid Interface Sci. 235 (2001) 194-196.

[25] G. Breuer, P.P. Lamers, D.E. Martens, R.B. Draaisma, R.H. Wiffels, The impact of nitrogen starvation on the dynamics of triacylglycerol accumulation in nine microalgae strains, Bioresour. Technol. 124 (2012) 217-226.

[26] Z. Wu, Y. Zhu, W. Huang, C. Zhang, T. Li, Y. Zhang, A. Li, Evaluation of flocculation induced by pH increase for harvesting microalgae and reuse of flocculated medium, Bioresour. Technol. 110 (2012) 496-502.

[27] L. Rodolfi, G.C. Zittelli, L. Barsanti, G. Rosati, M.R. Tredici, Growth medium recycling in Nannochloropsis sp. mass cultivation, Biomol. Eng 20 (2003) 243-248. 
[28] F. Hadj-Romdhane, X. Zheng, P. Jaouen, J. Pruvost, D. Grizeau, J.-P. Croue, P. Bourseau, The culture of Chlorella vulgaris in a recycled supernatant: effects on biomass production and medium quality, Bioresour. Technol. 132 (2013) 285-292.

[29] J.A. Choi, D.-Y. Kim, Y.H. Seo, J.-I. Han, Application of $\mathrm{Fe}\left(\mathrm{NO}_{3}\right)_{3}$-based as nitrogen source and coagulant for cultivation and harvesting of Chlorella sorokiniana, Bioresour. Technol. 222 (2016) 374-379.

[30] J.J. Milledge, S. Heaven, A review of the harvesting of micro-algae for biofuel production, Rev. Environ. Sci. Bio/Technol. 12 (2013) 165-178.

[31] R. Lemlich, Adsorptive bubble separation methods_-foam fractionation and allied techniques, Ind. Eng. Chem. 60 (1968) 16-29.

[32] S. Boonyasuwat, S. Chavadej, P. Malakul, J.F. Scamehorn, Surfactant recovery from water using a multistage foam fractionator: Part I effects of air flow rate, foam height, feed flow rate and number of stages, Sep. Sci. Technol. 40 (2005) 1835-1853.

[33] PubChem, Open Chemistry Database. https://pubchem.ncbi.nlm.nih.gov/compound/Poloxamer_188, (accessed 10 February 2018).

[34] L. Zhao, J. Du, Y. Duan, H. Zhang, C. Yang, F. Cao, G. Zhai, Curcumin loaded mixed micelles composed of Pluronic P123 and F68: preparation, optimization and in vitro characterization, Colloids Surf. B. Biointerfaces 97 (2012) 101-108.

[35] Y. Zheng, T. Li, X. Yu, P.D. Bates, T. Dong, S. Chen, High-density fed-batch culture of a thermotolerant microalga Chlorella sorokiniana for biofuel production, Appl. Energy 108 (2013) 281-287.

[36] M. Tredici, N. Bassi, M. Prussi, N. Biondi, L. Rodolfi, G.C. Zittelli, G. Sampietro, Energy balance of algal biomass production in a 1-ha "Green Wall Panel" plant: How to produce algal biomass in a closed reactor achieving a high Net Energy Ratio, Appl. Energy 154 (2015) 1103-1111.

[37] A. Guccione, N. Biondi, G. Sampietro, L. Rodolfi, N. Bassi, M.R. Tredici, Chlorella for protein and biofuels: from strain selection to outdoor cultivation in a Green Wall Panel photobioreactor, Biotechnol. Biofuels 7 (2014) 84.

[38] R. Bosma, J. De Vree, P. Slegers, M. Janssen, R. Wijffels, M. Barbosa, Design and construction of the microalgal pilot facility AlgaePARC, Algal Res. 6 (2014) 160-169.

[39] K.L. Mittal (Ed.), Polymer surface modification: relevance to adhesion, Vol. 3, CRC Press, Florida, 2004.

[40] M. Ishida, R. Haga, N. Nishimura, H. Matuzaki, R. Nakano, High cell density suspension culture of mammalian anchorage independent cells: Oxygen transfer by gas sparging and defoaming with a hydrophobic net, Cytotechnology 4 (1990) 215-225.

[41] C.-M. Chan, T.-M. Ko, H. Hiraoka, Polymer surface modification by plasmas and photons, Surf. Sci. Rep. 24 (1996) $1-54$.

[42] K. Tsougeni, P.S. Petrou, A. Tserepi, S.E. Kakabakos, E. Gogolides, Nano-texturing of poly (methyl methacrylate) polymer using plasma processes and applications in wetting control and protein adsorption, Microelectron. Eng. 86 (2009) 1424-1427.

[43] M. Sanchis, V. Blanes, M. Blanes, D. Garcia, R. Balart, Surface modification of low density polyethylene (LDPE) film by low pressure $\mathrm{O}_{2}$ plasma treatment, Eur. Polym. J. 42 (2006) 1558-1568.

[44] A. Skłodowska, M. Woźniak, R. Matlakowska, The method of contact angle measurements and estimation of work of adhesion in bioleaching of metals, Biol. Proced. Online 1 (1999) 114-121.

[45] Y.-P. Wang, K. Yuan, Q.-L. Li, L.-P. Wang, S.-J. Gu, X.-W. Pei, Preparation and characterization of poly (Nisopropylacrylamide) films on a modified glass surface via surface initiated redox polymerization, Mater. Lett. 59 (2005) 1736-1740.

[46] A. Moshonov, Y. Avny, The use of acetylene glow discharge for improving adhesive bonding of polymeric films, J. Appl. Polym. Sci. 25 (1980) 771-781.

[47] T. Zawodzinski, S. Gottesfeld, S. Shoichet, T. McCarthy, The contact angle between water and the surface of perfluorosulphonic acid membranes, J. Appl. Electrochem. 23 (1993) 86-88.

[48] J. Bongaerts, K. Fourtouni, J. Stokes, Soft-tribology: lubrication in a compliant PDMS-PDMS contact, Tribol. Int. 40 (2007) 1531-1542.

[49] S. Guruvenket, G.M. Rao, M. Komath, A.M. Raichur, Plasma surface modification of polystyrene and polyethylene, Appl. Surf. Sci. 236 (2004) 278-284.

[50] B. Yang, X. Duan, J. Huang, Ultrathin, Biomimetic, Superhydrophilic Layers of Cross-Linked Poly (phosphobetaine) on Polyethylene by Photografting, Langmuir 31 (2015) 1120-1126.

[51] V. Goetz, F. Le Borgne, J. Pruvost, G. Plantard, J. Legrand, A generic temperature model for solar photobioreactors, Chem. Eng. J. 175 (2011) 443-449. 
[52] A.M. Abdel-Ghany, I.M. Al-Helal, S.M. Alzahrani, A.A. Alsadon, I.M. Ali, R.M. Elleithy, Covering materials incorporating radiation-preventing techniques to meet greenhouse cooling challenges in arid regions: a review, Sci. World J. 2012 (2012) 906360.

[53] J. Pruvost, B. Le Gouic, O. Lepine, J. Legrand, F. Le Borgne, Microalgae culture in building-integrated photobioreactors: Biomass production modelling and energetic analysis, Chem. Eng. J. 284 (2016) 850-861.

[54] E. Molina, J. Fernández, F. Acién, Y. Chisti, Tubular photobioreactor design for algal cultures, J. Biotechnol. 92 (2001) 113-131.

[55] H. Qiang, Y. Zarmi, A. Richmond, Combined effects of light intensity, light-path and culture density on output rate of Spirulina platensis (Cyanobacteria), Eur. J. Phycol. 33 (1998) 165-171.

[56] C. Schreiber, D. Behrendt, G. Huber, C. Pfaff, J. Widzgowski, B. Ackermann, A. Müller, V. Zachleder, Š. Moudřiková, $P$. Mojzeš, Growth of algal biomass in laboratory and in large-scale algal photobioreactors in the temperate climate of western Germany, Bioresour. Technol. 234 (2017) 140-149.

[57] B. Podola, T. Li, M. Melkonian, Porous Substrate Bioreactors: A Paradigm Shift in Microalgal Biotechnology?, Trends Biotechnol. 35 (2017) 121-132.

[58] J. Pruvost, F. Le Borgne, A. Artu, J. Legrand, Development of a thin-film solar photobioreactor with high biomass volumetric productivity (AlgoFilm@) based on process intensification principles, Algal Res. 21 (2017) 120-137.

[59] T. Naumann, Z. Çebi, B. Podola, M. Melkonian, Growing microalgae as aquaculture feeds on twin-layers: a novel solid-state photobioreactor, J. Appl. Phycol. 25 (2013) 1413-1420.

[60] T.E. Murphy, H. Berberoglu, Flux balancing of light and nutrients in a biofilm photobioreactor for maximizing photosynthetic productivity, Biotechnol. Progr. 30 (2014) 348-359.

[61] J. Doucha, K. Lívanský, Productivity, $\mathrm{CO}_{2} / \mathrm{O}_{2}$ exchange and hydraulics in outdoor open high density microalgal (Chlorella sp.) photobioreactors operated in a Middle and Southern European climate, J. Appl. Phycol. 18 (2006) 811-826.

[62] G. Olivieri, L. Gargiulo, P. Lettieri, L. Mazzei, P. Salatino, A. Marzocchella, Photobioreactors for microalgal cultures: a Lagrangian model coupling hydrodynamics and kinetics, Biotechnol. Progr. 31 (2015) 1259-1272.

[63] L.K. Schultze, M.-V. Simon, T. Li, D. Langenbach, B. Podola, M. Melkonian, High light and carbon dioxide optimize surface productivity in a Twin-Layer biofilm photobioreactor, Algal Res. 8 (2015) 37-44.

[64] J. Doucha, K. Lívanský, Outdoor open thin-layer microalgal photobioreactor: potential productivity, J. Appl. Phycol. 21 (2009) 111-117.

[65] M. Morita, Y. Watanabe, H. Saiki, Evaluation of photobioreactor heat balance for predicting changes in culture medium temperature due to light irradiation, Biotechnol. Bioeng. 74 (2001) 466-475.

[66] N.S. Osborne, H.F. Stimson, D.C. Ginnings, Measurements of Heat Capacity and Heat of Vaporization of Water in the Range of $0^{\circ}$ to $100^{\circ} \mathrm{C}$, J. Res. Natl. Bur. Stand. 23 (1939) 197-260. 
Summary 
Microalgae are a promising feedstock for bulk materials, such as chemicals, fuels or feed constituents. However, currently the application of microalgae is limited to the production of high-value products because of the high production costs of algal biomass. In order to reduce the production costs of microalgae, we have developed a novel photobioreactor. This design is based on the application of liquid foams as a growth matrix for microalgal cells instead of a continuous liquid phase. Liquid foam-bed photobioreactors own several advantages over traditional, liquid phase based cultivation systems. Firstly, the high gas holdup of liquid foams leads to a reduced light absorption path in the foam-bed. Due to this short optical path, high biomass densities can be achieved, which results in the reduction of downstream processing costs of microalgal biomass. Secondly, the large interfacial area offered by liquid foams contributes to enhanced mass transfer rates. High mass transfer rates together with the low hydrostatic pressure of foam columns allow for a reduction in gassing energy requirements. Thirdly, in foam-beds, increased $\mathrm{CO}_{2}$ uptake efficiency can be achieved because of the long residence time of the gas phase in the reactor. Lastly, the low pressure drop in foam-bed photobioreactors also contribute to reduced material requirements for the construction of the photobioreactor.

The proof of principle of a liquid foam-bed photobioreactor is presented in Chapter 2. The most important aspect of the design of such a reactor was found to be foam formation and breakup. For foam formation, a surface active protein, Bovine Serum Albumin (BSA) was added to the microalgae suspension. Foam was created by homogenous distribution of small gas bubbles rising through a liquid phase containing surfactant. Due to the continuous gas supply, the created foam-bed rises in the reactor. When the foam reached the top of the reactor, the gas phase entrapped in the foam bubbles needed to be refreshed in order to supply sufficient amount of $\mathrm{CO}_{2}$ for photosynthetic microalgal growth and to remove the accumulated $\mathrm{O}_{2}$. As such, the foam was transported from the top of the reactor towards a foam breaking device. In this external foam breaker, the foam bubbles were broken and the deliberated algal suspension was pumped back to the bottom of the reactor where it was incorporated again in freshly formed foams. For foam breaking, different methods were tested, and a packed bed with hydrophobic beads appeared to be the most suitable for the foam-bed photobioreactor. The optimal gas flow rate and the surfactant concentration in the reactor was experimentally determined to allow for the formation of a stable but breakable foam. The developed reactor enabled short-term microalgae cultivation. An average growth rate of $0.1 \mathrm{~h}^{-1}$ was achieved during 8 hours of batch reactor operation. Longer operation was not possible due to the degradation of the protein 
applied for foam stabilization. BSA showed rapid biodegradability by bacteria and also the protein molecules might have denatured due to the physical forces exerted by foaming.

The rapid degradability of BSA revealed the need for a surfactant that allows for long-term operation of the foam-bed photobioreactor. Therefore, in Chapter 3, we focused on the selection of a surfactant that enables foam stabilization for elongated time periods. Firstly, four criteria were identified for a suitable surfactant: i) it should have good foaming properties; ii) it should not be toxic to the microalgal cells; iii) it should not be rapidly biodegradable, iv) microalgal cells should be dragged up with the foams formed. Ten different surfactants were tested for these criteria. Amongst these surfactants, the non-ionic poloxameric surfactants exhibited good foam properties, low biodegradability and lack of toxicity. Overall, Pluronic F68 showed the best properties and was selected as the most promising surfactant for the foambed photobioreactor. However, the Pluronic formed foams had low microalgae content since the foams did not drag up algal cells efficiently.

In Chapter 4, we focused on the application of Pluronic F68 for foam stabilization in an optimized liquid foam-bed photobioreactor. We demonstrated that this surfactant can ensure long term-foam formation and therefore also long-term reactor operation. The low microalgae partitioning towards the Pluronic F68 stabilized foam phase was circumvented by introducing a liquid flow over the foam-bed. The microalgae suspension was pumped from the bottom of the reactor to the top, where it was distributed homogenously over the foam column and allowed to drain down through the foam-bed. This liquid recirculation resulted in a more wet foam with homogenous microalgae distribution in the reactor. The streams of the recirculating liquid falling on top of the foam bed also served as a foam breaking method, thereby eliminating the need for an external foam breaker. The application of Pluronic F68 and the improved photobioreactor design enabled us to further characterize and reveal the potential of foam-bed systems. During continuous cultivation of Chlorella spp., biomass densities between 20 and 25 $\mathrm{g} \mathrm{L}^{-1}$ were maintained for more than three weeks. A biomass productivity of $57 \mathrm{~g} \mathrm{~m}^{-2} \mathrm{~d}^{-1}$ was achieved even though operational parameters such as dilution rate and liquid recirculation rate were not optimized. The mass transfer rate in the reactor was an order of magnitude higher than in bubble column reactors. The improved reactor design revealed the potential of liquid foambed reactors such as the possibility to achieve high biomass densities, long gas residence times, and enhanced mass transfer rates. 
In order to quantify the cost reduction related to biomass production in liquid foam-beds, a mathematical model was developed in Chapter 5. Algal growth was modelled in a continuously operated flat panel foam-bed photobioreactor at constant biomass densities. Similarly to the reactor design in Chapter 3, liquid recirculation was also incorporated in the modelled photobioreactor. Firstly, the liquid fraction distribution in height was calculated as a prerequisite for the determination of the light penetration. Based on the light availability in the photobioreactor, the microalgal growth and reactor productivity was predicted. Lastly, the gas transfer rates and $\mathrm{CO}_{2}$ requirements were calculated to ensure light limited algal growth. The liquid fraction and light model were experimentally validated. Model calculations showed that the areal productivity of the foam-bed photobioreactors are slightly lower compared to those of flat panels, which is related to light scattering in foams. However, in liquid foam-beds the biomass specific energy requirement for microalgae production and separation was calculated to be only $8.5 \%$ of those in flat panel photobioreactors. The reduced energy requirements are related to the increased biomass density and gas transfer rate in the foam-bed photobioreactor. The high biomass densities (30-60 $\left.\mathrm{g} \mathrm{L}^{-1}\right)$ allow for the reduction of downstream processing costs, and the large surface area contributes to increased gas transfer rates and thereby to a reduction in gas supply rate. In addition, a high $\mathrm{CO}_{2}$ uptake efficiency of $97 \%$ ensures that minimal $\mathrm{CO}_{2}$ is lost. The reduced energy requirements for biomass production highlighted the potential of liquid foam-bed photobioreactors as an economically feasible microalgae production system.

In Chapter 6, the obtained results were discussed and the application of foam-beds at large scale was evaluated. The most important aspects of liquid foam-bed photobioreactors, such as foam formation and foam break-up were discussed in detail, and suggestions were given to improve those. For example, we showed that the orifice radius of the gas distributor plate has to be increased to lower the pressure drop. Additionally, increased foam breaking efficiency achieved by the combination of hydrophobic solids and liquid jets as foam breaking methods can eliminate $\mathrm{pH}$ fluctuations and thereby improve productivity. We also concluded that the implementation of reduced liquid recirculation rates is essential for achieving low biomass specific energy requirements. We showed that surfactant re-use by medium recycling can reduce the biomass production costs and that a cooling chamber integrated on one side of the reactor is a prerequisite to for adequate control of the culture temperature. Additionally, different advanced photobioreactor designs were compared with the liquid foam-bed 
photobioreactor. Based on this comparison we concluded that liquid foam-beds can offer a cost effective large-scale microalgae production platform. 
Acknowledgements 
I had a great time during my $\mathrm{PhD}$, and I would like to thank to everyone who were part of my life during these years and who had contributed to that!

René, thank you for the possibility for doing my $\mathrm{PhD}$ at BPE! Also, thank you for your supervision: the long, useful and decisive meetings, proposition discussions and comments on my manuscripts. Next to our official meetings, I enjoyed your friendly attitude on the conference in China and on other social activities.

Marcel, thank you for your patience, trust and all the freedom you have given me during my $\mathrm{PhD}$. You have taught me a lot from the moment I started until the last days of my project. I learned a lot from you scientifically, but also in writing and presenting, and maybe also some organization skills :). Thank you also for the relaxed atmosphere on our biweekly meetings, and your friendly, direct and personal approach.

Maria V., it was super nice to work in such a close collaboration with you!!! Our PhD brought us pretty close, and we became friends really soon! Thanks for all the writing \& lab-help, talks, adventures, and all the fun trips and meetings!

Maria C., and Carlos, it was great to work closely together with you! Thanks for all the input in the project, and also thanks for all the relaxed and fun meetings!

Frederik, thank you for your help with the Foamscan! Ashok, thanks for your answers and ehelp! Packo, Karin Schroen, Joris Sprakel, and colleagues from Surfix, thank you for the initial meetings.

Eric, Kyle and the whole WUR workshop, and Fred and Sebastian, you deserve special thanks you, since without your input my $\mathrm{PhD}$ would surely not have been possible. I really relied on all your technical help! Also, I am grateful to Peter de Graaf for making the self-design glass reactor for me.

And my dear BPE colleagues, thank you a lot for making the everyday life colourful!

All stuff members, Arjen, Marian, Corjan, Mathieu, Ruud, Maria B., Giuseppe, Rouke, Miranda, Marina, Dirk, Packo, Douwe, Mark, Gerrit, Michel, Hans, and technicians, Fred, Sebastian, Snezana, Wendy, Rick, Bregje, coffee, cake, lunch and borrel times were always nice!! Arjen, thanks for always dropping by, talking and cheering me up :D 
Thanks Swimmers (Carl, Joao, Enrico, Fabian, Cata, Edgar), runners (Joao, Josue) and TRX-ers (Enrico, Cata, Alex, Edgar, Pieter, Narcis) for supporting my health during my $\mathrm{PhD}$ !:-)

And, thanks to Worms-ers (Ward, Josue, Joao, Youri) and Dinners/Beers group, although our programs weren't always that healthy ;P , but I had a lot of fun with you guys!!! Firstly the older colleagues: Abdu, Luci, Jeroen, Joao, Richard, Ward, Tim, Guido, Josue, Kiira, Lenny, Anne, Rafa, Carl, Giulia, Lenneke, Kim, Marjon, and the current ones: Alex, Anne, Cata, Elisa, Enrico, Fabian, Filippo, Kylie, Edgar, Wendy, Youri, Stephanie, Camilo, Calvin, Christian, Iris, Jorijn, Marta Sa, Narcis, Pauline, Pieter, Robin, Rocca, Sebastian, Iago, August, Renske!!

Thanks for the girls + dinner group for all the chatty evenings, attraction park and cooking tutorials: Mitsue, Jorijn, Marta, Ilse, Rupali, Xiao, Joao, Gerard, Gosia, Ruchir! Thanks for the nice Dutch evenings Joao, Mitsue and Hester. And thanks dear officemates: Youri, Joao, Abdu, Richard, Kylie, Calvin, Stephan, for creating a nice atmosphere, taking me tea, all the talks when we did not feel like working, and surviving my mess!:)

Jorijn, it was great to start at the same time with you and share all our experience during the $\mathrm{PhD}$. All our common travel was really nice, including the rooftop pool in Seville and such hard-working adventures:)! Mitsue, thanks for all the lunches, dinners and chats! And also for the princess double bed in Portugal:) Joao, you are a funny guy and was always interesting to listen your weird stories in the office. And, also was fun to be at your grandpa's house in Algarve;-)! Kylie, my first female officemate, thanks for the deep talks and understanding!! Cata, bioprocess design would not have been the same without you! Ward, you helped me with lab-stuff a bit, right? Thanks :P and also thanks for cooking us before Worms and entertaining me by being lame on the slackline at Madness:) Youri, I think I had the most beers with you during my PhD :) Thanks for all the advices, funny parties and continuous motivation for social activities! Iris, you have just arrived but I immediately feel like I know you for ever! Thanks for always being there for me for a great talk!

MIRACLES group! It was a fantastic adventure to work with you. All meetings were not only constructive, but really fun! Thanks for all scientific discussions and thanks even more for the memorable meetings Yolanda, Pia, Jorijn, Carl, Hanna, Marias \& Hans x2, Bienve, Rut, Joan and all others!! 
Monika, Yenny, Iulian, Soraya, Tom, Hahn-brothers, Nynke, Plub, Sanne, Hannes, Heike, Robin, Milan, Elise, Luuk, Davide, Marion, Bianca, and all slackers of Madness/ DTRH crew, although out of the university environment, you really taught me how to work efficient, as you always provided extremely nice free time activities, for what I was super motivated :-) Flo, thanks for your support, nice times, and the pictures too ;-)

Alex, Yorick, Vasilis, Simon, Rian, Rob, Piotr, Robin and Sam. Thanks a lot for your hard work contributing to this thesis!! I hope that the reactor blow-up story turned into a funny memory to learn from instead of a sad and scary event =). It was great to work with all of you, thanks again!

Thanks you, my Hungarian friends crew for all common trips and travels! Thanks my dear family for all the endless support! Anyu, Apu, David, Anna, Linda, Milla, Nagyi es Pipa!!! Koszonom szepen vegtelen tamogatasotokat mindenben!!! Pipa, a sok mateknak nagyban hasznat vettem, koszonom szepen turelmed es energiad! Anyu es Apu, a segitsegetek es tamogatasotok nelkul nem lennek most doktor...sott, meg Hollandiaig sem vittem volna! Millio puszi es koszonet mindenert! 


\section{About the author}

Ágnes Jánoska was born on the $20^{\text {th }}$ of October 1988 in Marcali, Hungary. She started her BSc studies in the field of biotechnology in 2007 at Budapest University of Technology and Economics (BME). In parallel, she studied as a mathematics teacher at Eötvös Loránd University in Budapest, Hungary. She did an internship at

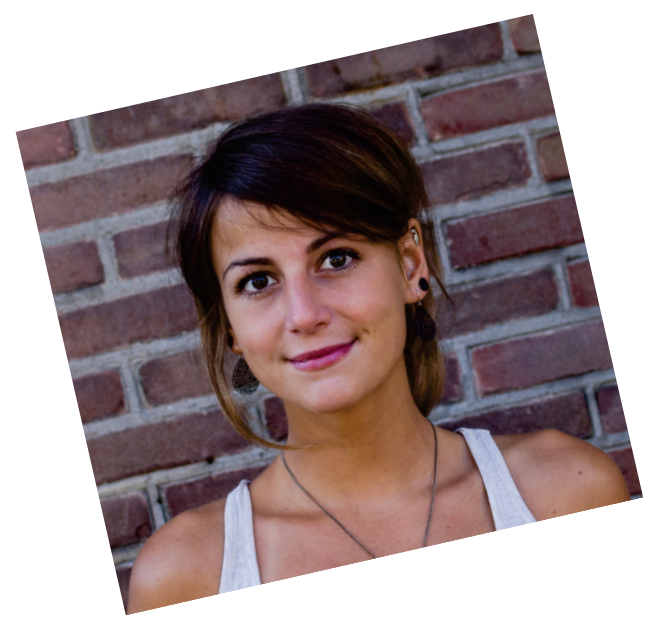
at Wetsus, European centre of excellence for sustainable water technology, in Leeuwarden, Netherlands, where she worked on microbial electrolysis cells, and the microbial examination of the biofilms responsible for hydrogen production. After obtaining her Bachelor degree in 2011, she decided to continue her studies in The Netherlands and she started her MSc studies of Water Technology at Wetsus. Her master thesis was entitled 'Anaerobic dimethyl disulphide removal at natron-alkaline conditions', where she operated anaerobic bioreactors for the removal contaminants from the gas phase. She also completed a minor thesis related to algal biofilms for wastewater treatment. She extended her water technology studies with additional biotechnology courses where she specialized on process technology. She graduated as Master of Science in biotechnology, at Wageningen University, in 2013. In the same year she started her $\mathrm{PhD}$ project on the development of a liquid foam-bed photobioreactor for microalgae cultivation at Wageningen University, bioprocess engineering chair group. Her project was part of the MIRACLES European Union project, and she worked in close collaboration with University of Huelva. During her $\mathrm{PhD}$, she developed a novel photobioreactor design based on liquid foams, and she characterized its potential via detailed experimental and modelling work. 


\section{List of publications}

A. Janoska, M. Janssen, R. H. Wijffels, Improved foam-bed photobioreactor design for microalgae cultivation, Manuscript submitted.

A. Janoska, M. Janssen, R. H. Wijffels, Potential of a liquid foam-bed photobioreactor for microalgae production, Manuscript submitted.

A. Janoska and M. Vázquez, M. Janssen, R. H. Wijffels, M. Cuaresma, C. Vílchez, Surfactant selection for a liquid foam-bed photobioreactor, Biotechnol. Prog. doi: 10.1002/btpr.2614

A. Janoska, P.P. Lamers, A. Hamhuis, Y. van Eimeren, R.H. Wijffels, M. Janssen, A liquid foam-bed photobioreactor for microalgae production, Chem. Eng. J. 313 (2017) 1206-1214

N. C. Boelee M. Janssen, H. Temmink, L. Taparavičiūtè, R. Khiewwijit, A. Janoska, C. J. N. Buisman, R. H. Wijffels, The effect of harvesting on biomass production and nutrient removal in phototrophic biofilm reactors for effluent polishing, J. Appl. Phycol. 26 (2014) 1439-1452. 


\section{Overview of completed training activities}

\section{Discipline specific}

Microalgae Process Design: from cells to photobioreactors (Wageningen, 2014)

Bioprocess Design (Delft, 2015)

\section{Conferences}

$4^{\text {th }}$ International CeBiTec Research Conference (Bielefeld, Germany, 2014) ${ }^{2}$

Young Algaeneers Symposium (Montpellier/Narbonne, France, 2014)²

European roadmap for an algae-based industry (Olhao, Portugal, 2016) ${ }^{2}$

$4^{\text {th }}$ Asia- Oceania Algae Innovation Summit (Wuhan, China, 2016) ${ }^{1}$

Algae Biorefineries for Europe (Brussels, Belgium, 2017)²

MIRACLES Project Meetings (Heelsum, Cadiz, Madrid, Antwerp, Las Palmas, Bergen, Faro, Bonn, Thessaloniki, 2013-2017) ${ }^{1}$

\section{General}

Vlag PhD week (Baarlo, 2014)

Project and time management (Wageningen, 2014)

The essentials of scientific writing and presenting (Wageningen, 2015)

Basic Statistics (Wageningen, 2016)

Scientific writing (Wageningen, 2016)

Carrier perspectives (Wageningen, 2017)

\section{Optionals}

Writing of a Research proposal (Wageningen, 2014)

$\mathrm{PhD}$ excursion (Portugal, 2014)

Group meetings and $\mathrm{PhD}$ days (Wageningen, 2013-2017)

\footnotetext{
${ }^{1}$ Oral presentation

2 Poster presentation
} 
This project has received funding from the European Union's Seventh Framework Programme for research, technological development and demonstration under grant agreement no 613588 . 



\section{Propositions}

1. Unwanted foams in traditional photobioreactors can be turned into attractive foam-bed photobioreactors. (this thesis)

2. Liquid foam-bed photobioreactors will become the cheapest production technology. (this thesis)

3. An environmental license on sustainable household practices should be obtained before buying or renting a house.

4. Freedom in organizing scientific data is more time-efficient for the researchers than forced data management plans.

5. Slacklining in work-breaks is more relaxing than drinking coffee.

6. Changing bad habits is harder than learning new things correctly.

Propositions belonging to the thesis, entitled

"A liquid foam-bed photobioreactor for microalgae production"

Ágnes Jánoska

Wageningen, $15^{\text {th }}$ June, 2018 